\title{
Lepidopterorum Gatalogus
}

editus a

\section{H. Wagner}

\section{Pars 7:}

\section{H. Zerny}

Syntomidae.

Einzel-Preis: Mark 16.90

Subscriptions-Preis!: Mark 11.25

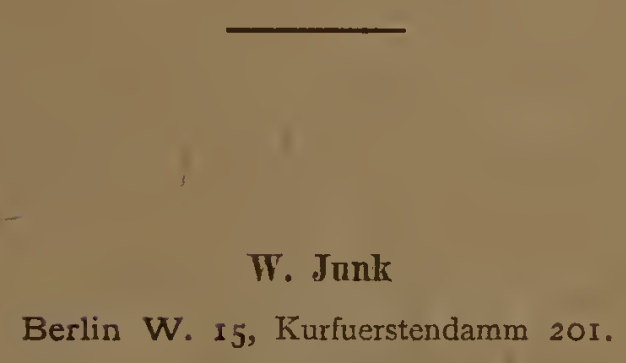

25. VII. 1912. 
W. JUNK, Berlin W. 15.

Verlag und Antiquariat fur Entomologie.

\section{Coleopterorum Catalogus}

Auspiciis et auxilio

W. Junk

editus a

S. Schenkling.

Bisher erschien: Published till now: Paru jusqu'aujourd'hui:

Partes 1-44. 1910-1912. (M. 371,50) Mark 247,80.

Disqué, Verzeichnis der in der Pfalz rorkommenden Kleinschmetterlinge (Micro-Isepidoptera). 1906.8 .73 p. Mk. 2.-

Federley, Lepidopterologische T'emperatur-Experimente mit besond. Beriicksichtigung der Flügelschuppen. 1905. 8. 119 p. m. 3 Tfln, u. 7 Fig. Mk. 5.-

Hartmann, Die Klein-Schmetterlinge (Nicro-Lepidoptera) des Europaeischen Faunengebietes. 1880. 8. $182 \mathrm{p}$.

(Mark 4.20) Mk. 2.50

Jensen, Bestemmelses-Tabeller or. Danske. Sommerfugle (MacroLepidopt.). 1902. 8. 23 p. m. Fig. Mk. 1.-

Junk, Entomologen-Adressbuch. The Entomologist's Directory. Annuaire des Entomologistes. 1905. 8. 302 p. Origbd. (Statt 5 Mark.).

MIk, 3.-

Circa 9000 Adressen aller Entomologen auf der ganzen civilisierten Erde nebst Angabe der Specialität enthaltend. Von der Fachpresse auf das glänzendste recensiert.

Mitterberger, Verzeichnis der im Kronlande Salzburg bisher beobachteten Microlepidopteren (Kleinschmetterlinge). 1909. 8. $358 \mathrm{p}$.

Nlk. $10 .-$

Prochnow, Die Laut-Apparate der Insekten. Beitr. zur Zoophysik u. Descendenz-Theorie. 1908. 8. 178 p. mit 49 Fig.

Mk. 5.-

Proclinow, Reactionen auf Temperatur-Reize. 1908. 8. 63 p. mit Fig.

Mk. 2.50

Ribbe, Anleitung zum Sammeln ron Schmetterlingen in tropischen Ländern. 1907. 8. 44 p.

Mk. 1.50 


\section{Lepidopterorum Catalogus}

editus a

\section{H. Wagner}

Pars 7:

H. Zerny

Syntomidae.

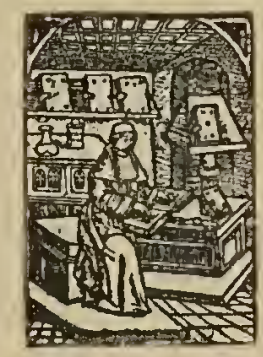

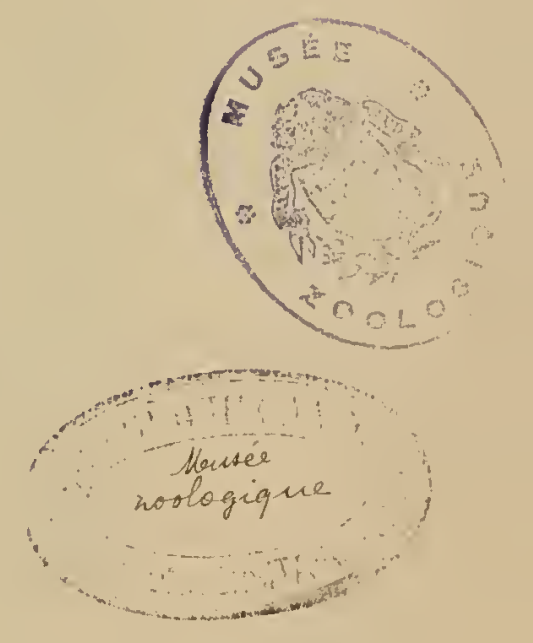

W. Junk

Berlin W. I5, Kurfuerstendamm 201. 1912. 



\section{Fam. Syntomidae.}

Auct. H. Zerny.

Syntomides》Herr.-Schäff., Syst. Bearb. Schmett. Eur. 2 (1), p. 4951 (1847).

Syntomoidea Herr.-Schäff., Aussercur. Schmett. p. 5, 21-23, $72-74$ (1854).

Syntomides +Charideoideae Wallengr., Öfvers. Svenska Akad. Förh. 19, p. 195, 197, (1862).

Hexaneuridae Wallengr., Svenska Akad. Handl. 5 (4), p. 50—51 (1865).

Syntomina Herr.-Schäff., Corr.-Bl. zool. min. Ver. Regensb. 20, p. 106-107 (1866).

Syntomidae Snell., Vlind. v. Nederl. 1, p. 128-129 (1867). Meyr., Proc. Linn. Soc. New South Wales 1 (2), p. 773-788 (1886). - Hamps., Moths Ind. 1, p. 209-228 (1892): 4, p. 465 (1894). - Neum. \& Dyar, Journ. New York Ent. Soc. 1, p. 100106 (1893). - Hamps., Cat. Lep. Phal. 1, p. 20-559, t. 1-17 (1898). - Turner, Proc. Roy. Soc. S. Austr. 22, p. 90-100, t. 5 (1898). — Dudgeon, Journ. Bombay Nat. Hist. Soc. 12, p. 292-294 (1899). - Hamps., ib. 13, p. 45-51, 223-224 (1900); Ann. S. Afr. Mus. 2, p. 35-41 (1900). - Schrottky, Iris 15, p. 133-136 (1902). - Holl., Moth Book, p. 98-103 (1903). - Hormuzaki, Ubers. pal. Lep.-Fam. p. 48, f. 25 (1904). - Turner, Proc. Linn. Soc. New South Wales 29, p. $831-859$ (1905); 31, p. 678-679 (1907). - Klages, Proc. U. S. Nat. Mus. 29, p. $531-552$ (1906). Spul., Schmett. Eur. 2, p. 126-128, f. 32 (1906). — Sharp, Cambr. Nat. Hist., Ins. 2, p. 388-390, f. 194 (1909). - Schrottky, Iris 22, p. 122-132 (1909); 24, p. 148-153 (1910). - Berge-Rcbel, Schmetterlingsb. p. 423-124 (1910). - Seitz, Grossschmett. d. Frde, F. pal. 2, p. 37-12, t. 9 (1910).

Zygaenidae p. p. (Subf. Syntominae, Thyretinae, Euchromiinae, Eunomiinae, Antichlorinae) + Arctiidae p. p. (Subf. Charideinae, Ctenuchinae) Butl., Journ. Linn. Soc. Lond., Zool. 12, p. 343-430 (1876). - Druce, Biol. Centr.-Am., Het. 1, p. $42-88$ (1884); 2, p. 332-36+ (1896-97).

Glaucopidae Burm., Descr. Phys. Rep. Argent. 5, p. 369-390 (1878).

Glaucopidae (Subf. Syntomina, Glaucopina) + Arctioida p. p. (Subf. Charideina) Plötz, Mitt. naturw. Ver. Neuvorpomm. 17 , p. 12-13 (1886).

Zygaenidae p. p. (Subf. Syntominae) Swinh., Cat. Lep. Het. Oxf. 1 , p. $40-55$ (1892).

Automolidinae Auriv., Ent. Tidskr. 13, p. 189 (1892).

Kataloge: Kirby, Cat. Lep. Het. p. 89-110, 117-180, 194-224 (1892) (Zygaenidae p. p. + Arctivdae p. p.). - Dyar, Cin. Ent. 
31, p. 158-160 (1899). - Stgr. \& Rbl., Cat. Lep. pal. Faunengeb. 1, p. 363-364 (1901). — Dyar, Bull. U. S. Nat. Mus. 52, p. 76-79 (1902).

An atomie: Fr. Müller, Jen. Zeitschr. f. Naturw. 11, p. 111 (1877) (Duftorgane). - Haase, Iris 1, p. 164 (1857) (Duftorgane).

Biologie: Seitz, Stett. Ent. Zeitg. 51, p. 260 (1890) (Mimicry). Hahncl, Iris 3, p. 140, 147, 161, 195, 239, 289, 308, (1890). Haase, Bibl. Zool. 8 (2), p. $73-77$ (1893) (Mimiory). - Pack. Mem. Acad. Philad. 7 (1), p. 79-81 (1895) (Puppe). - Hamps., Cat. Lep. Plial. 1, p. 22, f. 7, 8 (1898). - Spul., Schmett. Eur. 2, p. 126-128 (1906). — Sharp, Cambr. Nat. Hist., Ins. 2, p. 388390 (1909). - Schrottky, Iris 22, p. 122-132 (1909) (Mimicry). - Berge-Rebel, Schmetterlingsb. p. 423-424 (1910). - Seitz, Grossschmett. d. Erde, F. pal. 2, p. 37-42 (1910).

\section{Psychothoe Boisd.}

(Psichotoe) Boisd., Mon. Zyg. p. 129 (1829). - Butl., Journ. Linn. Soc. Lond., Zool. 12, p. 354, t. 28, f. 7 (1576). - Hamps., Moths Ind. 1, p. 225 (1892); Cat. Lep. Phal. 1, p. 32 (1898).

Cacosoma Feld., Reiso Nov. Lep., Atlas, p. 2, (1869).

Duvauceli Boisd., Mon. Zyg. p. 129, t. 8, f. 5, 6 (1829). - Spéc. Gén. Lép. t. 16, f. 6 (1858). - Butl.,

Sind bis Journ. Linn. Soc. Lond., Zool. 12, p. 354 (1876). - Hamps., Moths. Ind. 1, p. 226, f. 141 (1892); Cat. Lep. Phal. 1, p. 32, f. 9 (1898).

gnatula Boisd., Voy. Delegorguc 2, p. 596 (1847) (Naclia). - Wallengr., Svenska Akad. Handl. ó (4), p. 13 (1865). - Hamps., Cat. Lep. Phal. 1, p. 32, f. 10 (1898).

naclioides Feld., Reiso Nov, Lep. 2, p. 2, t. 102, 2 f. 13 (1869) (Ćacosoma).

Burma

Transvaal, Natal, Kapland

\section{Callitomis Butl.}

Butl., Journ. Linn. Soc. Lond., Zool. 12, p. 351, t. 27, f. 1 (1876). - Hamps., Moths Ind. 1, p. 224 (1892); Cat. Lep. Phal. 1, p. 33 (1898). - Piep. \& Snell., Tijdschr. v. Ent. 47, p. 45 (1904). — Scitz, Grossschmett. d. Erde, F. pal. 2, p. $41(1910)$.

distorta Rothsch., Nov. Zool. 17, p. 429 (1910).

Dohertyi Hamps., Cat. Lep. Phal. 1, p. 33, t. 1, f. 2 (1898). - Snell., Tijdschr. v. Ent. 47, p. 45 (1904).

Fruhstorferi Hamps., Cat. Lep. Phal. 1, p. 33, f. 11 (1898).

gigas Rotshch., Nov. Zool. 17, p. 429 (1910).

leucosoma Butl., Journ. Limn. Soc. Lond., Zool. 12, p. 351 (1876). - Hamps., Moths Ind. 1, p. 225 (1892); Cat. Lep. Phal. 1, p. 34, t. 1, f. 3 (1898). - Seitz, Grossschmett. d. Erde, F. pal. 2, p. 41, t. 9 h, (1910).

multifaseiata Hamps., Moths Ind. 1, p. 225 (1892); Cat. Lep. Phal. 1, p. 33, t. 1, f. 1, (1898).

J. Pulo Bisa bei Obi

Java

Celebos

Luzon

Kashmir

Sikkim, Assam 
syntomoides Butl., Journ. Linn. Soc. Lond., Zool. 12, p. 351 (1876). - Hamps., Moths Ind. 1, p. 224,

Kashmir, f. 139 (1892); Cat. Lep. Phal. 1, p. 34, f. 12 Himalaya 7 (1898). - Scitz, Grossschmett. d. Erde, F. pal. 2, p. $41(1910)$.

\section{Eutomis Hübn.}

Hübn., Verz. bek. Schmett. p. 122 (1826). — Butl., Journ. Linn. Soc. Lond., Zool. 12, p. 344, t. 28, f. 12 (1876). - Hamps., Cat. Lep. Phal. 1, p. 34 (1898).

minceus Cram., Pap. Exot. 4, p. 109, t. 347, f. A (1782) Kapland (Sphinx). - Hamps., Cat. Lep. Phal. 1, p. 34, 1 f. 13 (1898).

\section{Ceryx Wallengr.}

Wallengr., Wien. Ent. Monatsschr. 7, p. 140 (1863); Svenska Akad. Handl. 5 (4), p. 13 (1865). - Hamps., Cat. Lep. Phal. 1, p. 35 (1898). - Piep. \& Snell., Tijdschr. v. Ent. 47, p. 46 (1904). - Turner, Proo. Linn. Soc. New South Wales 29, p. 836 (1905). - Seitz, Grossschmett. d. Erde, F. pal. 2, p. 41 (1910).

Agaphthora Meyr., Proc. Linn. Soc. Now South Wales 1 (2), p. 774 (1886).

Syntomoides Hamps., Moths Ind. 1, p. 209 (1892).

\section{a) Äthiopische Arten.}

- albimacula Walk., List Lep. Ins. Br. Mus. 1, p. 128 (1854) (Syntomis). - Butl., Illustr. Het. Br. Mus. 1, p. 18, t. 6, f. 9 (1877). - Hamps., Cat. Lep. Phal. 1, p. 45 (1898). - Auriv., Ark. Zool. 2 (4), p. 27 (1904).

macrospila Walk., List Lep. Ins. Br. Mus. 31, p. 67 (1864) (Syntomis).

ab. terminal is Walk., List Lep. Ins. Br. Mus. 1, p. 130 (1851) (Syntomis). - Butl., Illustr. Het. Br. Mus. 1, p. 17, t. 7, f. 1 (1877). Hamps., Cat. Lep. Phal. 1, p. 46 (1898).

ab. constricta Bntl., Mlustr. Het. Br. Mus. 1, p. 18, t. 7, f. 6 (1877) (Syntomis). - Hamps., Cat. Lep. Phal. 1, p. 46 (1898).

ab. cybelistes Holl., Psyche 6, p. 393 (1893) (Syntomis). - Hamps., Cat. Lep. Phal. 1, p. 46 (1898).

ab. leugalea Holl., Psyche 6, p. 375 (1893) (Syntomis). - Hamps., Cat. Lep. Phal. 1, p. 46 (1898). anthrnciformis Wallengr., Wien. Ent. Monatsschr. 4, p. 40 (1860) (Naclia); Srenska Akad. Handl. 5 (4), p. 14 (1865). - Hamps., Cat. Lep. Phal. 1 , p. 37 , f. $16(1898)$.

fuscicornis Wallengr., Wien. Ent. Monatsschr. 4, p. 40 (1860) (Naclia); Srenska Akad. Handl. $5(4)$, p. 14 (1865).

pygmula Oberth., Et. Ent. 3, p. 33, t. 3, f. 6 (1878) (Naclia).

Sierra Leone bis Congo, Fernando Po.

Kapland 
Crawshayi Hamps., Ann. \& Mag. Nat. Hist. 8 (7), p. 166 (1901).

elasson Holl., Psyche 6, p. 375 (1893) (Syntomis). Hamps., Cat. Lep. Phal. 1, p. 46, t. 1, f. 21 (1898).

flava Beth.-Bakcr, Ann. \& Mag. Nat. Hist. (8) 7, p. 530 (1911).

- hilda Ehrmann, Can. Ent. 26, p. 69 (1894) (Syntomis). Dyar, ih. 31, p. 88 (1899).

seminigra Holl., Ent. Jews 9, p. 11 (1898) (Syntomoides ). - Hamps., Cat. Lep. Phal. 1, p. 46, t. 1 , f. 20 (1898).

longipes Herr.-Sclıäff., Aussereur. Schmett. p. 72, f. 273 (1855) (Syytomis). - Hamps., Cat. Lep. Phal. 1, p. 47 (1898).

caryocatactes Wallengr., Wion. Ent. Monatsschr. 4, p. 39 (1860) (Syntomis); Svenska Akad. Handl. 5 (4), p. 12 (1865).

resecta Herr.-Schäff., Aussereur. Schmett. p. 72 , f. 269 (1855) (Syntomis). - Hamps., Cat. Lep. Phal. 1, p. 43 (1898).

semihyalina Kirby, Ann. \& Mag. Nat. Hist. 18 (6), p. 379, t. 19, f. 5 (1896) (Zygaena). - Hamps., Cat. Lep. Phal. 1, p. 47, t. 1, f. 23 (1898).

toxotes Hamps., Cat. Lep. Phal. 1, p. 45, t. 1, f. 19 (1898).

Brit.-

Ostafrika

Kamerun,

Franz.-

Congo

Angola

Liberia, Kamerun, Tanganyikasee

Natal

Südafrika (ubi?).

Brit.-

Ostafrika

Kapland

\section{b) Orientalische Arten.}

albipuncta Hamps., Ann. \& Nlag. Nat. Hist. 19 (7), p. $221(1907)$.

catena Wileman, The Ent. 43, p. 220 (1910) (Syntomoides).

chea Druce, Ann. \& Mag. Nat. Hist. 15 (6), p. 43 (1895) (Syntomis). - Hamps., Cat. J.cp. Phal, 1, p. 45, t. 1 , f. 17 (1898).

cherra Moore, Lep. Atkins. p. 12 (1879) (Syntomis). Hamps., Moths Ind. 1, p. 220 (1892); Cat. Lep. Phal. 1, p. 41, t. 1, f. 13 (1898).

quisqualis Swinh., Ann. \& Mag. Nat. Hist. 14 (6), p. 441 (1894) (Syntomoides). - Hamps., Moths Ind. 4, p. 465 (1896).

Claremonti Herl., C. R. Soc. Ent. Belg. 34, p. XVI (1890) (Syntomis). - Hamps., Cat. Lep. Phal. 1, p. 36, t. 1, f. 5 (1898). - Snell., Tijdschr. v. Ent. 47, p. 47 (1904).

decorata Walk., Journ. Linn. Soc. Lond., Zool. 6, p. 89 (1862) (Syntomis). - Hamps., Cat. Lep. Phal. 1, p. 41 , t. 1, f. 11 (1898).

florina Butl., Journ. Linn. Soc. Lond., Zool. 12, p. 350 (1876) (Syntomis).

diptera Fabr., Syst. Ent. p. 555 (1775) (Zygaena). Auriv., Ent. T'idskr. 18, p. 154 (1897). - Hamps., Cat. Lep. Phal. 1, p. 36, f. 15 (1898).

atereus Cram., Pap. Exot. 4, p. 240 , t. 400 , f. A (1782) (Sphinx). - Hamps., Moths Ind. 1, p. 223 (1892).

incipiens Walk., List Lep. Ins. Br. Mus. 31, p. 68 (1864) (Syntomis). - Hamps., Moths Ind. 1, p. 211 (1892).

Luzon

Formosa

Mindanao

Assam

Sumatra,

Java

Borneo

Südindien, Ceylon 
ab. brachypecten Hamps., Illustr. Het. Br. Mus. 9, p. 61, t. 157, f. 23 (1893) (Psychotoe); Moths Ind. 1, p. 211 (1892); Cat. Lep. Phal. 1, p. 37 (1898).

Dohertyi Rothsch., Nov. Zool. 17, p. 430 (1910).

Eriessoni Rothsch., Nov. Zool. 17, p. 430 (1910).

exapta Swinh., Cat. Het. Mus. Oxf. 1, p. 48 (1892) (Syntomis). - Hamps., Cat. Lep. Phal. 1, p. 42, t. 1, f. 15 (1898). - Snell., 'Tijdsch. v. Ent. 47 , p. 48 (1904).

expandens Walk., Journ. Linn. Soc. Lond., Zool. 6, p. 91 (1862) (Syntomis). - Hamps., Cat. Lep. Phal. 1, p. 47 , t. 1, f. 22 (1898).

ab. inaequalis Snell., Iris 8, p. 129 (1895) (Syntomoides). - Hamps., Cat. Lop. Phal. 1, p. 47 (1898). - Snell., Tijdschr. v. Ent. 47, p. 49 (1904).

fata Swinh., Cat. Iep. Mus. Oxf. 1, p. 48 (1892) (Syntomis ). - Hamps., Cat. Lep. Phal. 1, p. 42, t. 1, f. 14 (1898). - Snell., Tijdschr. v. Ent. 47, p. 47 (1904).

finitiun Wileman, The Ent. 43, p. 220 (1910) (Syntomoides).

Ilaviplagia Hamps., Cat. Lep. Phal. 1, p. 36, t. 1, f. 4, (1898).

Syntomoides spec. Semp., Schmett. Philipp. 2, p. 418,707 (1898).

ginorea Swinh., Ann. \& Mag. Nat. Hist. 14 (6), p. 441 (1894) (Eressa); Trans. Ent. Soc. Lond. t. 1, f. 8 (1895). - Hamps., Moths Ind. 4, p. 465 (1896); Cat. Lep. Phal. 1, p. 37 (1898).

Godarti(-il) Boisd., Mon. Zyg. p. 115, t. 7, f. 3 (1829) (Syntomis), - Hamps., Moths Ind. 1, p. 210 (1892); Cat. Lep. Phal. 1, p. 43 (1898).

libera Walk., List Lep. Ins. Br. Mus. 31, p. 78 (1894) (Syntomis).

Fytchei Moore, Proc. Zool. Soc. Lond. p. 246, t. 18, f. 3 (1871) (Syntomis); in Anderson, Researches in West. Yunnan, p. 928, t. 81, f. 6 (1878).

cupreipennis Butl., Journ. Iinn. Soc. Lond., Zool. 12, p. 347 (1876) (Syntomis).

Hageni Snell., Iris 8, p. 129 (1895) (Syntomoides).

flavibasis Hamps., Cat. Lep. Phal. 1, p. 41, t. 1, f. 12 (1898).

- Lyalina Moore, Lep. Atkins. p. 13 (1879) (Syntomis). - Hamps., Moths Ind. 1, p. 211 (1892); Cat. Lep. Phal. 1, p. 42 (1893).

volans Stwinh., Trans. Ent. Soc. p. 173 , t. 6, f. 6

(1890) (Syntomis). - Hamps., Moths Ind. 1, p. 211 (1892).

inuon Cram., Pap. Fxot. 3, p. 94, t. 248, f. E (1780)

$(\operatorname{Sphinx}) .-$ Boisd., Mon. Zyg. p. 114 (1829). - Hamps., Moths Ind. 1, p. 210, f. 137 (1892); Cat. Lep. Phal. 1, p. 44, f. 18 (1893). - Dudgeon, Journ. Bombay Nat. Hist. Soc. 12, p. 292 (1899). - Snell., Tijdschr. v. Ent. 47, p. 48 (1904). - Seitz, Grossschmett. d. Erde, F. pal. 2 , p. 41 , t. 9 , h (1910).

\author{
Malakka \\ Sumatra \\ Java, \\ Lombok, \\ Sumbawa, \\ Flores \\ Perak, \\ Sumatra, \\ Java, \\ Borneo
}

Borneo, Java

Formosa

Mindanao

Assam

Sikkim u.

Südindien

bis

Yunnan

u. Sumatra,

Ceylon

Perak,

Sumatra

Sikkim,

Assam.

Burma

Südchina,

Vorder- 4 .

Hinter. :

indien,

Sumatra.

Java 
fusiformis Walk., List Lep. Ins. Br. Mus. 7, p. 1595 (1856) (Syntomis).

approximata Walk., List. Lep. Ins. Br. Mus. 31, p. 79 (1864) (Syntomis).

ab. sargania Butl., Trans. Ent. Soc. Lond. p. 4 (1879) (Syntomis). - Hamps., Cat. Lep. Phal. 1, p. 44 (1898). - Seitz, Grossschmett. d. Erde, F. pal. 2 , p. 41 (1910).

ab. artina Butl., Journ. Iinn. Soc. Lond., Zool. 12, p. 347 (1876) (Syntomis). - Moore, Lep. Ceyl. 2, p. 35, t. 94, f. 4 (1882). - Hamps., Cat. Lep. Phal. 1, p. 44 (1898). - Seitz, Grossschmett. d. Erde, F. pal. 2, p. 41 (1910).

ab. mota Swinh., Trans. Ent. Soc. Lond. p. 134, t. 8, f. 2, (1891) (Syntomis). - Hamps., Cat. Lep. Phal. 1, p. 44 (1898). - Seitz, Grossschmett. d. Erde, F. pal. 2, p. 41 (1910).

Biologie: Piepers Tijdschr. v. Ent. 47, p. 49 (1904). laterimacula Zerny nom. nov.

pleurosticta Hamps., Ann. \& Mag. Nat. Hist. 8 (7), p. 165 (1901); Journ. Bombay Nat. Hist. Soc. 16, p. 151, t. D, f. 2 (1904) (nec pleurosticta Hamps. (1898)).

Macgregori Schultze, Philipp. Journ. Sc. (Gen. Sc.) 3, p. 29 , t. 1 , f. 7 (1908).

pleurosticta Hamps., Cat. Lep. Phal. 1, p. 35, f. 14 (1898).

semieiueta Hamps., Trans. Ent. Soc. Lond. p. 282 (1895) (Syntomoides); Moths Ind. 4, p. 465 (1896); Cat. Lep. Phal. 1, p. 43 , t. 1, f. 16 (1898).

- transitiva Walk., Journ. Linn. Soc. Lond., Zool. 6, p. 89 (1862) (Syntomis). - Hamps., Cat. Lep. Phal. 1, p. 45 , t. 1 , f. 18 (1898).

intermissa Walk, Journ. Linn. Soc. Lond., Zool. 6, p. 90 (1892) (Syntomis).

ab. basalis Walk., List Lep. Ins. Br. Mus. 31, p. 79 (1864) (Syntomis). - Hamps., Cat. Lep. Phal. 1, p. 45 (1898).

\section{c) Australischo Arten.}

affinis Rothsch., Nov. Zool. 17, p. 429 (1910).

ampla Walk., List Lep. Ins. Br. Mus. 31, p. 76 (1864) (Syntomis). - Hamps., Cat. Lep. Phal. 1, p. 38, t. 1, f. 7 (1898).

aroa Beth.-Baker, Nov. Zool. 11, p. 382 (1904).

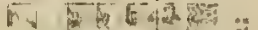

aurantiobasis Rotliseh., Nov. Zool. 17, p. 430 (1910).

evar Pagenst., Jahrb. Nass. Ver. Naturk. 39, p. 119 (1886) (Syntomis). - Hamps., Cat. Lep. Phal. 1, p. 40, t. 1 , f. 10 (1898).

gracilis va lugens Röber, Tijdsch. v. Ent. 34, p. 325 (1891) (Syniomis). - Hamps., Cat. Lep. Phal. 1, p. 537 (1898).

exapta Swinh. (cf. p. 7).

Vordcrindien:

Kanara

Philippinen

Borneo, Labuan

Tenasserim, Malakka, Borneo

Borneo, Malakka, Sumatra

Sumatra

Brit.-

Neuguinea

Aru-I.

Brit.-

Holl.

Neuguinea

Neuguinea

Amboina,

Aru-I.,

Key-I.

Goram J.

Java,

Lombok,

Sumbawa,

Flores 
Iormicina Swinh., Cato Het. Mus. Oxf. 1, p. 47 (1892) (Syntomis). - Hamps., Cat. Lep. Phal. 1, p. 39, f. 17 (1898).

guttulosa Walk., List. Lep. Ins. Brit. Mus. 31, p. 73 (1864) (Symtomis). - Swinh., Cat. Het. Mus. Oxf. 1, p. 46 (1892). - Hamps., Cat. Lep. Phal. 1, p. 39, t. 1, f. 8 (1898). - Turner, Proc. Linn. Soc. New South Wales 29, p. 836 (1905). melanora Meyr., Proc. Linn. Soc. New South Wales I (2), p. 774 (1886) (Agaphthora).

3 metan Pagenst., Jahrb. Nass. Vcr. Naturk. 39, p. 119 (1886) (Syntomis).

keiensis Rothsch., Nov. Zool. 17, p. 430 (1910).

Kuehui Rothsch., Nov. Zool. 17, p. 430 (1910).

Meeki Rothsch., Nov. Zool. 17, p. 429 (1910).

puncta Druce, Ann. \& Mag. Nat. Hist. 1 (7), p. 147 (1898) (Agaphthora). - Hamps., Cat. Lep. Phal. 1, p. 39 , t. 1 , f. 9 (1898).

sphenodes Meyr., Proc. Linn. Soc. Netw South Wales 1 (2), p. 774 (1886) (Agaphthora). - Hamps., Cat. Lep. Phal. 1, p. 38, t. 1, f. 6 (1898). Turner, Proc. Linn. Soc. New South Wales 29, p. 836 (1905).

Swinhoei Beth.-Baker, Nov. Zool. 11, p. 383 (1904).

v. minor Rothsch., Nov. Zool. 17, p. 431 (1910).

47

Holl. -

Neuguinea, etc.?

Aru-I.,

Key-I.,

Cap York

Key-I.

I. Taam b.

Key

Brit.-

Neuguinea

Holl.-

Neuguinea

Neuguinea

(ubi?),

Queensland

Brit.-

Neuguinea

Molukken:

Manavolka

Brit.

Neuguinca

1

\section{Iyopsyche Hamps.}

Hamps., Cat. Lep. Phal. 1, p. 48 (1898).

? Alluaudi Oberth., Ann. Soc. Ent. France 79, p. 467, t. 16, f. 6 (1911) (Naclia) (huius generis?).

? blandina Oberth., Et. Ent. 18, p. 1, t. 1, f. 7 (1893) (Naclia). - Hamps., Cat. Lep. Phal. 1, p. 50 (1898) (huius generis?).

cytogaster Holl., Psyche 6, p. 393 (1893) (Syntomis). Hamps., Cat. Lep. Phal, 1, p. 48, f. 19 (1898).

elachlsta Holl., Psyche 6, p. 375 (1893) (Syntomis). Hamps., Cat. Iep. Phal. 1, p. 51, t. 1, f. 28 (1898). ilda Plötz, Stett. Ent. Zeitg. 41, p. 79 (1880) (Symlomis). - Hamps., Cat. Lep. Phal. 1, p. 51, t. 1, f. 27 (1898). - Auriv., Ark. Zool. 2 (4), p. 28 (1904).

- miserabilis Holl., Psyche 6, p. 375 (1893) (Syntomis). Hamps., Cat. Lep. Phal. 1, p. 48, f. 20 (1898). notoplagla Hamps., Cat. Lep. Phal. 1, p. 49, t. 1, f. 26 (1898).
Madagaskar

,

Westafrika

(Taluguga)

Franz.-

Congo

Kamerun

Franz.-

Congo

Sierra Leone, Goldkiisto 
Ochsenheimeri Boisd., Mon. Zyg. p. 116, t. 7, f. 4 (1829) (Syntomis). - Hamps., Cat. Lcp. Phal. 1, p. 50, f. 21 (1898). - Auriv., Ark. Zool. 2 (4), p. 27 (1904).

ponga Plötz, Stett. Ent. Zeitg. 41, p. 78 (1880) (Syntomis).

puncticineta Holl., Psyche 6, p. 375 (1893) (Syntomis). - Hamps., Cat. Lep. Phal. 1, p. 49, t. 1, f. 24 (1898).

victorina Plötz, Stett. Ent. Zeitg. 41, p. 78 (1880) (Syntomis). - Hamps., Cat. Lep. Phal. 1, p. 51, f. 22 (1898).

xanthopleura Holl., Ent. News 9, p. 11 (1898) (Synto. moides). - Hamps., Cat. Lep. Phal. 1, p. 49, t. 1, f. 25 (1898).

xantlıosoma Hamps., Ann. \& Mag. Nat. Hist. 19 (7), Uganda 12 p. $222(1907)$.

Streptophlebia Hamps.

Hamps., Cat. Lep. Phal. 1, p. 52 (1898).

obliquistria Hamps., Cat. Lep. Phal. 1, p. 52, f. 23 (1898). Borneo

1

\section{Thylacoptera Hamps.}

Hamps., Cat. Lep. Phal. 1, p. 52 (1898).

albipuneta Hamps., Cat. Lep. Phal. 1, p. 53, f. 24 (1898).

1

\section{Symphlebomis Hamps.}

Hamps., Ann. \& Mag. Nat. Hist. 15 (7), p. 425 (1905).

antipolo Semper, Schmett. Philipp. 2, p. 418, t. 53, f. 2, 3 Luzon 1

(1898) (Syntomoides).

\section{Trichaeta Swinh.}

Swinh., Cat. Het. Mus. Oxf. 1, p. 52 (1892). - Hamps., Moths Ind. 1, p. 225 (1892); Cat. Lep. Phal. 1, p. 53 (1898). - Piep. \& Snell., Tijdschr. v. Ent. 47 , p. 50 (1904).

\section{a) Äthiopische Arten.}

bivittała Walk., List Lep. Ins. Br. Mus. 31, p. 66 (1864) (Syntomis). - Hamps., Cat. Lep. Phal. 1, p. 55 , t. 2, f. 2 (1898).

- Iulvescens Walk., List Lep. Ins. Br. Mus. 1, p. 132 (1854) (Syntomis). - Butl., Illustr. Het. Br. Mus. 1 , p. 17, t. 7, f. 2 (1877). - Hamps., Cat. Lep. Phal. 1, p. 54, f. 27 (1898).

caffraria Herr.-Sehäff., Aussereurop. Schmett. p. 72 , f. 271 (1855) (Syntomis).

Sierra Leone

Kapland bis Somaliland und Congo 
thyretiformis Wallengr., Wien. Ent. Monatsschr. 4, p. 40 (1880) (Naclia); Svenska Akad. Handl. $5(4)$, p. 14 (1865).

molanna Wallengr., Öfvers. Svenska Akad. Förh. 32 (1), p. 94 (1876) (Syntomis).

Biologic: Barrett, Ent. Monthl. Mag. 12 (2), p. 193 (1901).

parra Auriv., Ann. Mus. Genova 4 (3), p. 507 (1910).

- pterophorina Mab., Bull. Soc. Ent. France p. 139 (1892) (Syntomis). - Mab. \& Vuill., Nov. Lep. p. 152, t. 21, f. 7 (1895). - Hamps., Cat. Lep. Phal. 1, p. 53, f. 25 (1898).

Sehultzei Auriv., Ark. Zool. 2 (12), p. 24 (1905).

Portug.-Senegambion

Natal, Mashona-

land, Rhodesia,

Congo

Adamaua

\section{b) Orientalische Arten.}

albifrontulis Pagenst., Jahrb. Nass. Ver. Naturk. 38, p. 13, t. I, f. 8 (1885) (Syntomis). - Hamps., Cat, Lep. Phal. I, p. 56 (1898) (basifera Wlk. var.?). v. borealis Rothsch., Nov. Zool. 19, p. 123 (1912). albiplaga Walk., Journ. Linn. Soc. Lond., Zool. 6, p. 92 (1862) (Syntomis). - Hamps., Cat. Lep. Phal. 1, p. 57, f. 28 (1898).

v. albosignata Walk., List Lep. Ins. Br. Mus. 31, p. 71 (1864) (Syntomis). - Hamps., Cat. Lcp. Phal. 1. p. 57 (1898).

apicalis Walk., List Lep. Ins. Br. Mus. 7, p. 1626 (1856) (Tipulodes). - Hamps., Cat. Lep. Phal. 1, p. 58, t. 2 , f. $10,(1898)$.

biplagata Snell., in Veth, Midd. Sumatra, Lcp. p. 33 (1880) (Synlomis); Iris 8, p. 129 (1895).

v. flaviplaga Walk., Journ. Linn. Soc. Lond., Zool. 6, 1. 92 (IS62) (Syntomis). - Hamps., Cat. Lep. Phal. I, p. 58 (1898).

hasifera Walk., Journ. Linn. Soc. Lond., Zool. 6, p. 92 (1862) (Syntomis). - Hamps., Cat. Lep. Phal. 1, p. 57 , t. 2 , f. 6 (1898).

biplagiata Rothsch., Nov. Zool. 19, p. 123 (1912).

detracta Walk., Journ. Linn. Soc. Lond., Zool. 6, p. 91 (1862) (Syntomis). - Hamps., Cat. Lep. Phal. 1, p. 56, t. 2 , f. 4 (1898).

diplaga Hamps., Cat. Lep. Phal. 1, p. 58, t. 2, f. 9 (1898).

divisurn Walk., Journ. Linn. Soc. Lond., Zool. 1, p. 90 (1862) (Syntomis). - Hamps., Cat. Lep. Phal. 1, p. 59, t. 2, f. Il (1898).

elongimacula Hamps., Cat. Lep. Phal. 1, p. 54, t. 2, f. 1 (1898).

Hosei Rothsch., Nov. Zool. 17, p. 431 (1910).

Kannegieteri Rothsch., Nov. Zool. 17, p. 431 (1910).

monolenea Hamps., Ann. \& Mag. Nat. Hist. 11 (7), p. 337 (1903).

Nias

Borneo

"

Malakka

Malakka (M.

C. Vind.),

Sumatra

Borneo

Borneo, Malakka

Borneo

,

Penang,

Sumatra

(M. C.

Vind.),

Borneo

(M.C.Vind.)

Borneo

I. Pulolaut

b. Bornco

Borneo

Nias

Singaporc 
proleuea Hamps., Ann. \& Mag. Nat. Hist. 11 (7), p. 337 (1903).

quadriplagata Snell., Iris 8, p. 129 (1895) (Syntomis). Hamps., Cat. Lep. Phal. 1, p. 57, t. 2, f. 7 (1898). separabilis Walk., Journ. Linn. Soc. Lond., Zool. 6, p. 90 (1862) (Syntomis). - Hamps., Cat. Lep. Phal. 1 , p. 59 , t. 2 , f. 8 (1898).

ab. chloroleuca Walk., Journ. Linn. Soc. Lond., Zool. 3, p. 183 (1859) (Syntomis). - Hamps., Cat. Lep. Phal. 1, p. 59 (1898).

teneiformis Walk., List Lop. Ins. Br. Mus. 7, p. 1595 (1856) (Syntomis). Hamps., Moths Ind. 1, p. 225 , f. 140 (1892); Cat. Lep. Phal. 1, p. 58, f. 29 (1898).

tigrina Walk., List Lep. Ins. Br. Mus. 31, p. 71 (1864) (Syntomis). - Hamps., Cat. Lep. Phal. 1, p. 54, f. 26 (1898).

trizonata .Hamps., Cat. Lop. Phal. 1, p. 56, t. 2, f. 5 (1898).

Vigorsi Moore, Lep. East Ind. Comp. p. 323 (1859) (Syntomis); Proc. Zool. Soc. Lond. p. 198, t. 60, f. 4 (1859). - Hamps., Cat. Lep. Phal. 1, p. 56, t. 2, f. 3 (1898). - Snell., Tijdschr. v. Ent. 47, 24 p. 50 (1904).
Sumatra

Singapore, Borneo, I. Pulolaut b. Borneo

West-

himalaya,

Sikkim,

Bhutan

Cambodja

Malakka

Java

\title{
Syntomis Ochs.
}

Ochs., Eur. Schmett. 2, p. 103 (1808). - Boisd., Mon. Zyg. p. 107 (1829). - Hcrr.-Schäff., Syst. Bearb. Eur. Schmett. 2 (1), p. 49; 6, t. 13, f. 41 (1847-56). - Snell., Vlind. Nederl. 1, p. 129 (1867). - Moore, Lep. Ceyl. 2, p. 34 (1882). - Hamps., Moths Ind. 1, p. 212 (1892). - Comstock, Evol. \& Tax. p. 61, f. 11 (1893). - Hamps., Cat. Lep. Phaj. 1, p. 59 (1898) Piep. \& Snell., Tijdschr. v. Ent. 47, p. 51 (1904). - Turner, Proc. Linn. Soc. New South Wales 29, p. 837 (1905). - Spul., Schmett. Eur. 2, p. 127, f. 32 (1906). - Scitz, Grossschmott. d. Erde, F. pal. 2, p. 38 (1910). Berge-Rebel, Schmotterlingsb. p. 424, f. 103 (1910).

Amata Fabr., in Illiger, Magaz. 6, p. 289 (1807). - Prout, The Ent. 37, p. 116 (1904). - Rothsch., Nov. Zool. 17, p. 431 (1910) (nomen prius, sed non usitatum).

Gla ucopis Hübn., Tent. p. 1 (1805) (neo Gmcl. 1788).

Coenochromia Hübn., Verz. bek. Schmett., p. 121 (1826). Herr.-Schäff., Corresp.-Bl, zool.-min. Ver. Regensb. 20, p. 107 (1866). - Snell., Tijdschr, v. Ent. 22, p. 70 (1879).

Hydrusa Walk., List Lep. Ins. Br. Mus. 1, p. 255 (1854). - Meyr. Proe. Linn. Soc. New South Wales 1 (2), p. 774 (1886). - Turner Proc. Roy. Soc. S. Austr. 22, p. 92 (1898).

A sinusca Wallengr., Öfvers. Svenska Akad. Förlı. 19, p. 197 (1862); Svenska Akad. Handl. 5 (4), p. 13 (1865).

Asinutea Wallengr., Wien. Ent. Monatsschr. 7, p. 139 (1863).

Buthysia Wallengr., Wien. Ent. Monatsschr. 7, p. 139 (1863).

a) Äthiopische Arten.

alicia Butl., Journ. Linn. Soc. Lond., Zool. 12, p. 348 (1876) (Zygaena). - Hamps., Cat. Lep. Phal. 1, p. 83 , t. 3, f. 4 (1898).

\author{
Abyssinien, \\ Somaliland, \\ Adamaua, \\ Angola (M. \\ C. Vind.), \\ Südafrika \\ (M.C.Vind.)
}


v. apicalis Strand, Ent. Rundsch. 26, p, 109 (1909).

Biologie: ?Auriv., Ark. Zool. 2 (12), p. 25 (1905). atricornis Wallengr., Wien. Ent. Monatsschr. 7, p. 139

(1863) (Asinusca); Svenska Akad. Handl. 5 (4),

p. 13 (1865). - Hamps., Cat. Lep. Phal. 1, p. 75, t. 3 , f. 2 (1898).

sirius Dist., Ann. \& Mag. Nat. Hist. 20 (6), p. 15 (1897).

bilaseinta Hopff., Monatsber. Akad. Berl. p. 422 (1857); in Peters, Reisc Mossambique, Ins. p. 426, t. 27, f. 14 (1864). - Hamps., Cat. Lep. Phal. 1, p. 108 (1898).

borguensis Hamps., Ann. \& Mag. Nat. Hist. 8 (7), p. 166 (1901).

Burtti Dist., The Ent. 33, p. 261 (1900).

eacrulescens Druce, Ann. \& Mag. Nat. Hist. 1 (7), p. 146 (1898). - Hamps., Cat. Lep. Phal. 1, p. 113, t. 4 , f. 19 (1898).

cerbera L., Mus. Ulr. p. 363 (1764) (Sphinx). - Drury, III. Exot. Eilt. 1, p. 56, t. 26, f. 2 (1770). Cram., Pap. Exot. 1, p. 101, t. 83, f. F (1776). Boisd., Mon. Zyg. p. 118, t. 7, f. 6 (1829). Wallengr., Svenska Akad. Handl. 5 (4), p. 11 (1865). - Hamps., Cat. Lep. Phal. 1, p. 83 (1898). - Auriv., Ann. Mus. Genova 4 (3), p. $507(1910)$.

ab. phantasia (fantasia) Butl., Journ. Linn. Soc. Lond., Zool. 12, p. 349 (1876). - Hamps. Cat. Lep. Phal. 1, p. 83 (1898).

v. francisca Butl., Journ. Linn. Soc. Lond., Zool. 12, p. 349 (1876). - Hamps., Cat. Lep. Phal. 1, p. 83 (1898).

curtiplaga Mab., Ann. Soc. Ent. France 10 (6), p. 35 (1890).

ccres Oberth., Et. Lnt. 3, p. 33, t. 3, f. 5 (1878). - Hamps., Cat. Lep. Phal. 1, p. 82 (1898).

chloroscia Hamps., Ann. \& Mag. Nat. Hist. 8 (7), p. 168 (1901).

Cholmlei Hamps., Ann. \& Mag. Nat. Hist. 19 (7), p. 223 (1907).

chrysozona Hamps., Cat. Lep. Phal. 1, p. 90, t. 3, f. 21 (1898).

congenern (eongener) Hamps., Ann. \& Mag. Nat. Hist. 8 (7), p. 168 (1901).

consimilis Hamps., Ann. \& Mag. Nat. Hist. 8 (7), p. 167 (1901).

creobota Holl., Psyche 6, p. 393 (1893). - Hamps., Cat. Lep. Phal. 1, p. 101, t. 3, f. 28 (1898).

croceizona Hamps., Proc. Zool. Soo. Lond. p. 389, t. 36, f. $3(1910)$.

cuprizonata Hamps., Ann. \& Mag. Nat. Hist. 8 (7), p. 168 (1901).

Deutsch-

Ostafrika

Kapland, Transvaal, Mashona-

land

Mozambique

Nigeria, Adamaua

I. Pemba b.

Zanzibar

Delagoabai

Kapland bis

Somaliland

u. Portug.-

Senegam -

bien

Sierra Leone,

Gabun,

Congo,

Delagoabai

Zanzibar,

Brit.-

Zentral-

afrika

Deutsch- $u$.

Brit.-

Ostafrika

Brit.-

Ostafrika

Deutseh.

Ostafrika,

Somahland

Brit.-

Ostafrika

Deutseh- $u$.

Brit.-

Ostafrika

Franz.-Congo

Rhodesia

Brit.-

Ostafrika. 
damareusis Grünberg in Schultze, Forschungsreise Südafrika 4 (1), p. 122 , t. 3, f. $6(1910)$.

diiateralis Hamps., Cat. Lep. Phal. 1, p. 82, t. 3 , f. 3 (1898).

discata Druce, Ann. \& Mag. Nat. Hist. 1 (7), p. 147 (1898). - Hamps., Cat. Lep. Phal. 1, p. 112, t. 4 , f. 21 (1898).

dissimilis Beth.-Baker, Ann. \& Mag. Nat. Hist. 7 (8), p. 530 (1911).

divalis Schaus \& Cloments, Lep. Sierra Leone p. 21, t. 1, f. 2 (1893.) - Hamps., Cat. Lep. Pnal. 1, p. 84 (1898).

endoeroeis Hamps., Ann. \& Hag. Nat. Hist. 11 (7), p. 338 (1903).

Goodi Holl., Psyche 6, p. 376 (1893). - Hamps., Cat. Lep. Phal. 1, p. 65, t. 2, f. 18 (1898).

ab. Reutlingeri Holl., Psyche 6, p. 376 (1893). - Hamps., Cat. Lep. Phal. 1, p. 65 (1898).

lımiphoenlea Hamps., Proc. Zool. Soc. Lond. p. 389, t. 36, f. $1(1910)$.

interniplaga Mlab., Ann. Soc. Ent. France 10 (6), p. 35 (1890). - Hamps., Cat. Lep. Phal. 1, p. 114, t. 4 , f. 16 (1898).

Jaclisoni Rothsch., Nov. Zool. 17, p. 432 (1910) (Amata). johanna Butl., Journ. Linn. Soc. Lond., Zool. 12, p. 348 (1876). - Hamps., Cat. Lep. Phal. 1, p. 102, t. 4, f. 7 (1898).

ab. a nna Butl., Journ. Linn. Soc. Lond., Zool. 12, p. 348 (1876). - Hamps., Cat. Lep. Phal. 1, p. 102 (1898) (f. princip.).

Kenredi Rothsch., Nov. Zool. 17, p. 436 (1910) (Amata). Kuhlweiu i Lef., Mag. Zoul., Ins. t. 23 (1831). - Wallengr., Svenska Akad. Handl. 5 (4) p. 12 (1865). Butl., Illustr. Het. Br. Mus. 1, p. 16, t. 6, f. 17 (1877). - Hamps., Cat. Lep. Phal. 1, p. 82 (1898). - Auriv., Sjöstedt Exped. Kilim., Lep. p. 23 (1909).

Biologie: Barrett, Ent. Nonthl. Mag. 12 (2), p. 193 (1901).

iagosensis Hamps., Ann. \& Mag. Nat. Hist. 19 (7), p. 224 (1907).

lateralis Boisd., Spéc. Gén. Lép. 1, t. 12, f. 5 (1858). Hamps., Cat. Lep. Phal. 1, p. 111 (1898).

marginalis Walk., List Lep. Ins. Br. Mus. 1, p. 130 (1854). - Butl., Illustr. Het. Br. Mus. 1, p. 16 , t. 6 , f. 13 (1877).

ieucerythra Holl., Psyche 6, p. 376, 393 (1893). - Hamps., Cat. Lep. Phal. 1, p. 81 (1898).

rubicunda Mab., Bull. Soc. Ent. France p. 139 (1892). - Mab. \& Vuill., Nov. Lep. p. 153, t. 21, f. 8 (1895)

limacis (leimacis) Holl., Psyche 6, p. 375 (1893) - Hamps., Cat. Lep. Phal. 1, p. 61, f. 31 (1898).

- marina Butl., Journ. Linn. Soc. Lond. Zool. 12, p. 348 (1876). - Hamps., Cat. Lep. Phal. 1, p. 114, t. 4, f. 24 (1898).

negretina Plötz, Stett. Ent. Zeitg. 41, p. 78 (1880). ogovensis Holl., Psyche 6, p. 374 (1893).
DeutschSüdwestafrika

DeutschOstafrika "

Aequatoria

Sierra Leone

Mashonaland, Congo

Franz.Congo

Congo

Nigeria, Gabun, Congo

Uganda

Kapland, Adamaua

Congo

Kapland, Natal, Transvaal, DeutschOstafrika

Lagos

Senegal, Ashanti

Kamerun,

Gabun

(M.C.Vind.),

Congo (II. C. Vind.)

Franz.Congo

Sierra Leone bis Uganda u. Rhodesia 
miozona Hamps., Proc. Zool. Soc. Lond., p. 389, t. 36, f. 2 (1910).

monticola Auriv., Sjöstedt Exped. Kilim., Lep. p. 24, t. 1 , f. 9 (1909).

Natulii Boisd., Voy. Delegorgue 2, p. 596 (1847). Hcrr.-Schäff., Aussereur. Schmett. p. 72, f. 268 (1855). - Wallengr., Svenska Akad. Handl. 5 (4), p. 12 (1865). - Auriv., Sjöstedt Exped. Kilim, Lep. p. 23 (1909).

nigrobasalis Rotlisch., Nov. Zool. 17, p. 436 (1910) (Amata).

nigrieilia Strand, Arch. Naturg. 78, A, p. 82 (1912).

n'tebi Beth.-Baker, Ann. \& Mag. Nat. Hist. 7 (8), p. 531 (1911).

Pembertoni Rothsch., Nov. Zool.o 17, p. 431 (1910) (Amata).

phaeobasis Haunps., Ann. \& Mag. Nat. Hist. 19 (7), p. 223 (1907). - ? Grünberg, Sitzungsber. Ges. naturf. Fr. Berlin p. 156 (1910).

phaeozona Zerny, Iris 36 (1912) (ined.).

phoenieia Hamps., Cat. Lep. Phal. 1, p. 70, t. 2, f. 23 (1898).

Rendalli Dist., Ann. \& Mag. Nat. Hist. 20 (6), p. 16 (1897). - Hamps., Cat. Lep. Phal. 1, p. 112, t. 4 , f. 23 (1898).

rubritincta Hamps., Ann. \& Mag. Nat. Hist. 11 (7), p. 338 (1903).

rufina Oberth., Et. Ent. 3, p. 32, t. 3, f. 4 (1878). Hamps., Cat. Lep. Phal. 1, p. 84 (1898).

shoa Hamps., Cat. Lep. Phal. 1, p. 70, t. 2, f. 25 (1898).

simplex Walk., List Lep. Ins. Br. Mus. 1, p. 129 (1854). - Hamps., Cat. Lep. Phal. 1, p. 111 (1898).

monedula Wallengr., Wien. Ent. Monatsschr. 4, p. 39 (1860); Svenska Akad. Handl. 5 (4), p. 12 (1865).

ab. nostalis Walk., List Lep. Ins. Br. Mus. 1, p. 129 (185t). - Butl., lllustr. Het. Br. Mus. 1, p. 15 , t. 6, f. 11 (1877). - Hamps., Cat. Lep. Phal. 1, p. 111 (1898).

ab. corvus Wallengr., Wien. Ent. Monatsschr. 4, p. 39 (1860); Svenska Akad. Handl. כૅ (4), p. 12 (1865). - Hamps., Cat. Lep. Phal. 1, p. 111 (1898).

steuoptera Zerny nom. nov.

attenuata Rothsch., Nov. Zool. 17, p. 437 (1910) (Amata) (nec Hamps. 1901).

stictoptera Rothsch., Nov. Zool. 17, p. 432 (1910) (Amata).

thomasina (tomasina) Butl., Journ. Linn. Soc. Lond., Zool. 12, p. 348 (1876). - Hamps., Cat. Lep. Phal. 1, p. 84 , t. 3, f. 5 (1898).
Rhodesia;

Dtsch.-Ostafrika (var.)

Deutsch-

Ostafrika

Natal

Goldküste;

Uganda (var.)

Deutsch-

Ostafrika

Uganda

Angola

Uganda

DeutschOstafrika

Brit.Ostafrika

(J. Manda,

M'Kwajuni)

Transvaal

Brit.-

Ostafrika

Abyssinien

Nubien,

Abyssinien

Natal

Brit.-

Ostafrika

Elfenbein-

küste

Sierra Leone, Congo, Ostafrika (zw. Salzsee u. Wawamba) 
tripunctata Beth.-Baker, Ann. \& Mag.:Nat. Hist. 7 (8), p. 530 (1911). . +

tritonia Hamps., Ann. \& Mag. Nat. Hist. 8 (8), p. 394 (1911).

velatipennis Walk., List Lep. Ins. Br. Mus. 31, p. 67 (1864). - Hamps., Cat. Lep. Phal. 1, p. 84, t. 3, f. 6 (1898).

? veronica Oberth., Et. Ent. 18, p. 8, t. 1, f. 15 (1893) (Naclia). - Hamps., Cat. Lep. Phal. 1, p. 113 (1898) (huius generis?).

?ab. magdalene Oberth., Et. Ent. 18, p. 9, t. 1, f. 16 (1893) (Naclia). - Hamps., Cat. Lep. Phal. 1, p. 113 (1898).

Waldowi Grünberg, Deutsch. Ent. Zeitschr. p. 443, t. 4, f. 3 (1907).

Willani Rothsch., Nov. Zool. 17, p. 433 (1910) (Amata).

\section{b) Paläarktische Arten.}

alicia Butl. (cf. p. 12).

v. mogadorensis Blachier, Ann. Soc. Ent. France 77 , p. 219 , t. 41, f. 9 (1908).

antiochena Led., Wien. Ent. Monatsschr. 5, p. 151, t. 1, f. 13, 14 (1861). - Hamps., Cat. Lep. Phal. 1, p. 109 (1898). - Seitz, Grossschmett. d. Erde, F. pal. 2, p. 39, t. 9, d (1910).

ab. taurica Hamps., Cat. Lep. Phal. 1, p. 109 (1898). - Seitz, Grossschmett. d. Erde, F. pal. 2, p. 39 (1910).

bactrlana Ersch., Fedtsch. Reise Turkest. p. 29, t. 2, f. 24 (1874). - Hamps., Cat. Lep. Phal. 1, p. 109 (1898). - Scitz, Grossschmett. d. Erde, F. pal. 2, p. 39, t. 9, c (1910).

- caspla Stgr., Stett. Ent. Zeitg. 38, p. 176 (1877); Mém. Rom. 1, p. 82, t. 4, f. 7 (1884), - Alph., ib. 6, p. 4 (1892). - Hamps., Cat. Lop. Phal. 1, p. 108 (1898). - Spul., Schmett. Eur. 2, p. 127, t. 76, f. 5 (1906). - Seitz, Grossschmett. d. Erde, F. pal. 2 , p. 39 , t. 9 , d (1910).

gunssuensis Grum-Grshim., Hor. Soc. Ent. Ross. 25, p. 461 (1891). - Hamps., Cat. Lep. Phal. I, p. 110 (1898). - Seitz, Grossschmett. d. Erde, F. pal. 2 , p. $38(1910)$.

germana Feld. (of. p. 22).

libanotica Bang-Haas, Iris 19, p. 147 (1906). — Seitz, Grossschmett. d. Eirde, F. pal. 2, p. 39 (1910).

- maracandina Eirsch., Fedtsch. Reise Turkest., Lep. p. 30, t. 2, f. 25 (1874). - Hamps., Cat. Lep. Phal. 1, p. 108 (1898). - Seitz, Grossschmett. d. Firde, F. pal. 2 , p. 39 , t. 9 , o (1910).

ab. cocandica Ersch., Fedtsch. Reise Turkest., Lep. p. 31, t. 2, f. 26 (1874). - Hamps., Cat. Lep. Phal. 1, p. 108 (1898). - Seitz, Gross. schmett. d. Erde, F. pal. 2, p. 39 (1910).
Angola

S. Nigeria

Ayssinien,

Somaliland

Madagaskar

Adamaua

Brit.-

Ostafrika

Marokko

Syrien

Taurus,

Syrien

Sarawschan

Südost.

Russland,

Süd-Ural,

Armenien,

Tarbagatai

Kuku-Noor

Amur,

Ussuri,

Corea,

China,

Annam:

Japan

Syrien

Transkaspien Sarawscham 
Mestrali Bugnion, Ann. Soc. Ent. France 6, p. 439, t. 16, f. 1 (1837). - Led., Wien. Ent. Monatsschr. 5, p. 151 , t. 1, f. 11,12 (1861). - Hamps., Cat. Lop. Phal. 1, p. 109 (1898). - Scitz, Grosssclimett. d. Erde, F. pal. 2, p. 38, t. 9, c (1910). Kindermanni Led., Wien. Ent. Monatsschr. 2, p. 142 (1858).

ab. Palaestinae Hamps., Cat. Lep. Phal. 1, p. 110 (1898). - Scitz, Grossschmett. d. Erdc, F. pal. 2, p. 39 (1910).

minuta Bang-Haas, Iris 24, p. 49 (1911).

persica Koll., Denkschr. Akad. Wien, math.-nat. Cl. 1, p. $53(1850)$. - Hamps., Cat. Lep. Phal. 1, p. 101 , t. 4 , f. 5 (1898). - Seitz, Grossschmett. d. Erdc, F. pal. 2, p. 39, t. 9, f (1910).

phegea L. Syst. Nat. ed. 10, p. 494 (1758) (Sphinx). Drury, Ill. Exot. Ent. 1, p. 53, t. 25, f. 2 (1770); Esp., Schmett. 2, p. 144, t. 17, f. 1, 2 (1779). Hb., Eur. Schmett. 2, f. 99, 100 (1802-08). Ochs., Eur. Schmett. 2, p. 105 (1808). - God., Hist. Nat. Lép. 3, p. 154 , t. 22, f. 14 (1821). - Boisd., Mon. Zyg. p. 108, t. 6, f. 8 (1829). Herr.-Schäff., Syst. Bearb. Schmett. Eur. 6, t. 13, f. 41-45, t. 15 (1856). - Sncll., Vlind. Nederl. 1, p. 129 (1867); 2, p. 1137 (1882). Hamps., Cat. Lcp. Phal. 1, p. 110 (1898). - Spul., Schmett. Eur. 2, p. 127, t. 72 , f. 14 (1906). Seitz, Grossschmett. (1. Firdc, F. pal. 2, p. 38, t. 9, a (1910). - Berge-Rcbcl, Schmetterlingsb. p. 424 , t. 50, f. $10(1910)$. - Rammc, Int. Ent. Zcitschr. 4, p. 182 , f. $1-5$ (1910).

ligata Ill., Mél. Phill.-Math. Turin p. 193 (1766) (Sphinx).

quercus Fabr., Syst. Ent. 3 (1), p. 388 (1793) (Zygaena).

ab. eloelia Borkh., Naturg. cur. Schmett. 2, p. 117 (1789). - Lsp., Schmctt., Suppl. 2, p. 46 (1805). - Hamps., Cat. Lep. Phal. 1, p. 110 (1898). - Spul., Schmett. Eur. 2, p. 127 (1906). - Berge-Rebel, Schmetterlingsb. p. 424 (1910). - Seitz, Grossschmett. d. Erdc, F. pal. 2, p. 38, t. $9, \mathrm{~b}(1910)$.

phegea var., Esp., Schmett. 2, p. 220, t. 34, f. 1 (1779).

ab. cyclopea Ragusa, Nat. Sic. 17 , p. 20, t. 1, f. 3 (1905). - Seitz, Grossschmctt. d. Erde, F. pal. 2 , p. 38 , t. 9 , b (1910).

ab. fenestrata Ramme, Int. Ent. Zeitschr. 5, p. 103,125 , fig. (1911).

ab. iphimedea Esp., Schmett. Suppl. 2, p. 49, t. 47, f. $4(1805)(\operatorname{Sphin} x)$. - Hamps., Cat. Lep. Phal, 1, p. 110 (1898). - Spul., Schmett. Eur. 2, p. 127 (1906). - Berge-Rebel, Schmetterlingsb. p. 424 (1910). - Scitz, Grossschmett. d. Erdc, F. pal. 2, p. 38, t. 9, b (1910).

phegea Hb., Eur. Schmett. 2, f. 145 (1818-22). ab. Kruegeri Ragusa, Nat. Sic. 17, p. 20, t. 1, f. 2 (1905). - Seitz, Grossschmett. d. Erde, F. pal. 2 , p. 38 , t. 9 , a $(1910)$.

Syricn

Transkaspicn

Persien

Mittel- u. Südcuropa (excl.

Grossbritannien u. ibcr. Halbinsel), Siid-Ural, Kaukasus, Kleinasien, Arunenien, Altai 
ab. nigricornis Alph., Rev. Mens. Ent. St. Petersb. 1, p. 18 (1883). - Hamps., Cat. Lep. Phal. 1, p. 110 (1898). - Spul., Schmett. Eur. 2, p. 127 (1906). - Scitz, Grossschmett. d. Erde, F. pal. 2 , p. 38 (1910).

ab. orbiculifera Zerny, nom. nov.

phegea ab. Hafner, Grossschmett. Krains, p. 202, 237, t. 2, f. 127 (Carniola 1911-12).

ab. Pfluemeri Wacquant, Stctt. Ent. Zeitg. 37, p. 370 (1876). - Spul., Sehmett. Eur. 2, p. 127 (1906). - Berge-Rebel, Schmetterhingsb. p. 424 (1910). - Seitz, Grosssehmett. d. Erde, F. pal. 2, p. 38 , t. 9 , a $(1910)$.

ab. phegeus Esp., Sohmett. Suppl. 2, p. 48, t. 47 , f. 3 (1805). - Spul., Schmctt. Eur. 2, p. 127 (1906). - Berge-Rebel, Schmetterlingsb. p. 424 (1910). - Seitz, Grosssclimett. d. Erde, 1. pal. 2, p. 38 , t. 9 , b (1910).

phegea Hb., Eur. Sehmett. 2, f. 30 (1796).

ab. seminigra spul., Sehmett. Eur. 2, p. 127

(1906). - Bcrge-Rebel, Selimetterlingsb. p. 424 (1910).

ab. sexmaculala Gianelli, Nat. Sic. 17, p. 25 (1905).

Riologie: Oclıs., Eur. Sclimett. 2, p. 110 (1808). - Hübn., Eur. Sehmett. Larv. 2, 1, B, e. (1806-18). - God., Hist. Nat. Lep. 3, p. 154 (1821). - Guér., Icon. Régne Animal, Ins. p. 500 , t. 82, f. 7, 7a (1843). - Wilde, Raup. Deutschl. p. 104 (1861). - Sncll., Vlind. Nederl. 1, p. 129 (1867). - Praun, Eur. Sehmettcrlingsraup. t. 1, f. 7 (1872). - Dyar, Proe. Bost. Nat. Hist. Soc. 27, p. 138 (1896). - Hamps., Cat. Lep. Phal. 1, p. 110 (1898). - Gillm., Ill. Zeitschr. Ent. 5, p. 251 (1900). - Pabst, Kranch. Ent. Jalırb. 12, p. 15 (1903).- Spul., Schnett. Eur. 2, p. 127, 492, Raup. t. 10, f. $10 a, b$, t. 50, f. $25(1906-10)$. - Pirling, Int. Ent. Zeitsclir, 2, p. 27 (1908).

- Berge-Rebel, Schmetterlingsb. p. 424, t. 50, f. la, b (1910). - Seitz, Grossschmett. d. Erde, F. pal. 2 , p. $38(1910)$.

Slntenisi Standf., Mém. Rom. 6, p. 664, t. 15, f. 4 (1892).

- Hamps., Cat. Lep. Phal. 1, p. 108 (1S98). -

Seitz, Grossschmett. d. Erde, F. pal. 2, p. 39, t. 9 , d (1910).

trlcingulata Culot, Bull. Soc. Ent. France p. 271 (1909).

Kurdistan,

Sarawschan

\section{c) Orientalische Arten.}

acrosplla Feld., Reise Nov,, Lep. 2, p. 9, t. 102, f. 11 (1869). - Hamps., Cat. Lep. Phal. 1, p. 91 (1898). - Seitz, Grossschmett. d. Erde, F. pal. 2, p. 40 , t. 9 h $(1910)$.

ab. confluens Leech, Trans. Ent. Soc. Lond. p. 322 (1898).

a crospila ab. 1 Hamps., Cat. Lop. Phal. 1, p. 91 (1898).

Ostchina 
actea Swinh., Trans. Ent. Soc. Lond. p. 474, t. 19, f. 7 (1891) (Hydrusa); ib. p. 32 (1895). - Hamps., Cat. Lep. Phal. I, p. 64 (1898).

v. Swinhoei Leech, The Ent. 31, p. 152 (1898). - Seitz, Grossschmett. der Erde, F. pal. 2, p. 40 (1910).

actea subsp. 1 Hamps., Cat. Lep. Phal. 1, p. 64 (1898).

ab. obsoleta Lecch, The Ent. 31, p. 152 (1898). albapex Hamps., Illustr. Het. Br. Mus. 9, p. 62, t. 157, f. 1 (1893); Moths Ind. 1, p. 214 (1892); Cat. Lep. Phal. 1, p. 105 (1898).

albicornis Rothsch., Nov. Zool. 17, p. 433 (1910) (Amata). albilrons Moore, Proc. Zool. Soc. Lond. p. 845, t. 53, f. 6 (1878). - Hamps., Motlis Ind. 1, p. 216 (1892); Cat. Lep. Phal. 1, p. 100 (1898).

annulata Fabr. (of. p. 27).

assamensis Zerny, nom. nov.

khasiana Rothsch., Nov. Zool. 17, p. 435 (1910) (Amata) (nec Butl. 1876).

aurantiifrous Rothsch., Nov. Zool. 18, p. 154 (1911) (Amata).

aurea Swinh., Ann. Mag. Nat. Hist. (8) 1, p. 62 (1908).

anreola Swinh., Ann. \& Mag. Nat. Hist. (7) 9, p. 78 (1902).

bajaea Swinl., Trans. Ent. Soc. Lond. p. 473, t. 19, f. 10 (1891) (Hydrusa); ib. p. 32 (1895). - Hamps., Moths Ind. 1, p. 222 (1892); Cat. Lep. Phal. 1, p. 67 (1898).

berinda Moore, Proc. Zool. Soc. Lond. p. 845, t. 53, f. 8 (1878). - Hamps., Moths Ind. 1, p. 219 (1892); Cat. Lep. Phal. 1, p. 98 (1898).

bielneta Koll. in Hügel, Kaschmir 4, p. 460 , t. 19, f. 8 (1844). - Hamps., Moths Ind. 1, p. 213 (1892): Cat. Lep. Phal. 1, p. 106 (1898). - Seitz, Grossschmett. d. Erde, W. pal. 2, p. 38, t. 9, b (1910).

brithyris Druce, Ann. \& Mag. Nat. Hist. (7) 1, p. 146 (1898). - Hamps., Cat. Lep. Phal. 1, p. 90, t. 3 , f. 16 (1898).

Cantori Moore, Lep. East Ind. Comp. p. 326 (1859); Proc. Zool. Soc. Lond. p. 199, t. 60, f. 12 (1859). - Hamps., Cat. Lep. Phal. 1, p. 113 (1898).

chlorocera Hamps., Moths Ind. 1, p. 218 (1892); Cat. Lep. Phal. 1, p. 93, t. 3, f. 22 (1898).

cingulats Weber, Obs. Ent. p. 109 (1801) (Zygaena). - Hamps., Cat. Lep. Phal. 1, p. 107, t. 4, f. 17 (1898). - Seitz, Grossehmett. d. Erde, F. pal. 2, p. 39, t. 9, d (1910).

atereus Walk., List Lep. Ins. Br. Mus. 1, p. 128 (1854) (nec Cram.).

a nnetta Butl., Journ. Linn. Soc. Lond., Zool. 12, p. 347 (1876).

Fortunei Leech, Proo. Zool. Soc. Lond. p. 593 (1888) (p. p.).
Assam

West- und Nordostchnia

Ceylon

Tonkin

Burma

Philippinen

(Sambo-

angan),

Neuguinea,

Australien

Assam

Formosa

Assam

"

Assam,

Borneo,

Java

Burma

China,

Nordindien

Borneo

Penang

Assam

China 
compta Walk., Charact. Undeser. Het. p. 89 (1869). Hamps., Cat. Lep. Plial. 1, p. 99, t. 5, f. 1 (1898). - Dudgeon, Journ. Bombay Nat. Hist. Soe. 12 , p. 293 , t. 11, f. 24 (1S9y). - Hamps., ib. 13 , p. $50(1900)$.

connecteus Rothseh., Nor. Zool. 17, p. 433 (1910) (Amata). consequa Lcceh, The Ent. 31, p. 153 (1898). - Hamps., Cat. Iep. Pisal. 1, p. 96, t. 5, f. 2 (1898). Scitz, Grosssclumett. d. Erdc, F. pal. 2, p. 40, f. 9 , e $(1910)$.

cynatilis Swinh., Cat. Het. Mus. Oxf. 1, p. 40 (1892). - Hamps., Cat. Lep. Phal. 1, p. 76, f. 35 (1898). - Semp., Schmett. Philipp. 2, p. 419, t. 53, f. 4, 5 (1898).

laomedia Druce, Ann. \& Nag. Nat. Hist. (6) 15, p. 43 (1895).

cyssea(-us) Cram., Pap. Exot. 4, p. 124, t. 355, f. B (1782) (Sphinx). - Boisd., Mon. Zyg. p. 113, t. 7, f. 2 (1829). - Butl., Journ. Linn. Soe. Lond., Zool. 12, p. 343 (1876). - Hanps., Motlis Ind. 1, p. 213 , f. $13 S$ (1892). - Swinl., Trans. Ent. Soe. Lond. p. 30 (1895). - Hamps., Cat. Lep. Phal. 1, p. 105 , f. 36 (1898). - Scitz, Grossschmett. d. Ercle, F. pal. 2, p. 39, t. 9, e (1910).

collaris Fabr., Syst. Ent. 3 (1), p. 388 (1793) (Zygaena). - Auriv., Eint. Tidskr. 18, p. 153 (1897).

Schoenerrhi Boisd., Mon. Zyg. p. 112, t. 7, f. 1 (1829).

cuprea Prittw., Stett. Ent. Zeitg. 28, p. 277 (1867).

ab. georgina Butl., Journ. Linn. Soc. Lond., Zool. 12, p. 345 (1876). - Moore, Lep. Ceyl. 2, p. 34 , t. 95 , f. 2 (1882). - Hamps., Moths Ind. 1, p. 213 (1892); 4, p. 465 (1896); Cat. Lep. Phal. 1, p. 105 (1898).

ab. cysseoiles Butl., Journ. Linu. Soe. Lond., Zool. 12, p. 346 (1876). - Moore, Lep. Ceyl. 2, p. 35, t. 95, f. 1 (1882). - Hamps., Cat. Lep. Phal. 1, p. 105 (1598).

Biologie: Swinh., Proc. Zool. Soc. Lond. p. 155 (1883).

derivata Walk., Journ. Linn. Soe. Lond., Zool. 6, p. 89 (1862). - Hamps., Cat. Lep. Phal. 1, p. 60, t. 2, f. 12 (1898). - Snell., Tijdschr, v. Ent. 47 , p. 52 (1904).

diaphana Koll. in Hügel, Kasehmir 4, p. 460, t. 19 , f. 7 (1844). - Hamps., Moths Ind. 1, p. 216 (1892); Cat. Lep. Phal. 1, p. 67 (1898). - Snell., Tijdschr. v. Fint. 47 , p. 53 (1904). - Seitz, Grosssehmett. d. Erde, F. pal. 2, p. 40 (1910).

vitreata Herr.-Sehiaff., Aussereurop. Sclımett. p. 72, f. 267 (1855).

o enone Butl., Journ. Linn. Soe. Lond., Zool. 12, p. 344 (1876).

dich otoma Lceeh, The Ent. 31, p. 153 (1898). - Hamps., Cat. Lep. Phal. 1, p. 100, t. 4, f. 1 (1898). Seitz, Grosssehmett. d. Erde; F. pal. 2, p. 39, t. 9, e (1910).
Silkim,

Assam

Burma

Westehina

Philippinen

Vorderindien, Assam,

Ceylon

Perak,

Sumatra,

Java,

Borneo

West-

limalaya,

Assam,

Burma,

Java

Westehina 
ab. concurrens Lecch, The Ent. 31, p. 153 (1898). - Hamps., Cat. Lep. Phal. 1, p. 100 (1898). Seitz, Grosssclimett. d. Erde, 15. pal. 2, p. 39, t. 9 , e (1910).

dilatata Snell. in Veth, Midden Sumatra, Lep. p. 33 (1880). - Pagenst., Jahrb. Nass. Ver. Naturk. 38 , p. 12 , t. 1 , f. 6 (1885). - Hamps., Cat. Lep. Phal. 1, p. 87 (1898). - Snell., Tijdsehr. v. Ent. 47 , p. 54 (1904).

dirisa Walk., List Lep. Ins. Br. Mus. 1, p. 131 (1854). But1., Illustr. Het. Br. Mus. 1, p. 16, t. 6, f. 14 (1877). - Hamps., Motlıs Ind. 1, p. 215 (1892); Cat. Lep. Pinal. 1, p. 98 (1898).

ab. disrupta Moore, Proc. Zool. Soc. Lond. p. 845, t. 53, f. 5 (1878). - Hamps., Cat. Lep. Phal. 1, p. 98 (1898).

Edwardsi Butl., Journ. Linn. Soe. Lond., Zool. 12, p. 346 (1876). - Hamps., Cat. Lep. Phal. 1, p. 104, t. 4 , f. 11 (1898).

egenaria Walk., Journ. Linn. Soe. Lond., Zool. 6, p. 88 (1862). - Hamps., Cat. Lep. Phal. 1, p. 86, t. 3 , f. 9 (1898).

elisa Butl., Journ. Linn. Soc. Lond., Zool. 12, p. 347 (1876). - Hamps., Cat. Lep. Plial. 1, p. 61, f. $30(1898)$.

elongata Hamps., Journ. Bombay Nat. Hist. Soc. 13, p. $50(1900)$.

Elwesi Rothsch., Nov. Zool. 17, p. 432 (1910) (Amata). era Swinl., Trans. Ent. Soc. Lond. p. 473 , t. 19, f. 15 (1891) (Hydrusa). - Hamps., Moths Ind. 1, p. 222 (1892); Cat. Lep. Phal. 1, p. 68 (1898).

euryptera Hamps., Cat. Lep. Phal. 1, p. 87 , t 3, f. 10 (1898).

euryzona Leech, The Ent. 31, p. 153 (1898). - Hamps., Cat. Lep. Phal. 1, p. 94 , t. 3 , f. 19 (1898). Seitz, Grossselimett. d. Erde, F. pal. 2, p. 40 , t. $9, \mathrm{~g}(1910)$.

extensa Walk., List Lep. Ins. Br. Mus. 35, p. 1863 (1866). - Hamps., Mlustr. Het. Br. Mius. 8, t. 143, f. 14 (1890); Motlis Ind. 1, p. 214 (1892); Cat. Lep. Plial. 1, p. 103 (1898).

fervida Walk., List Iop. Ins. Br. Mus. 1, p. 131 (1854). - Butl., Illustr. Het. Br. Mus. 1, p. 18, t. 6, f. 16 (1877). - Hamps., Nloths Ind. 1, p. 217, (1892); Cat. Lep. Phal. 1, p. 93 (1898).

Ilavifrons Hamps., Moths Ind. 1, p. 215 (1892); Cat. Lop. Phal. 1, p. 101, t. 4, f. 3 (1898).

flaviguttata Hamps., Journ. Bombay Nat. Hist, Soc. 13, p. 50, t. B, f. 2 (1900).

flavolavata Rothsch., Nov. Zool. 17, p. 434 (1910) (Amata).

Formosae Butl., Journ. Linn. Soc. Lond., Zool. 12, p. 346 (1876). - Hamps., Moths Ind. 1, p. 220 (1892); Cat. Lep. Phal. 1, p. 98, t. 3, f. 26 (1898). Seitz, Grossschmett. d. Erde, F. pal. 2, p. 39, t. 9 , e (1910).

emma Butl., Journ. Linn. Soc. Lond., Zool. 12, p. 350 (1876).

\section{Sumatra, \\ Nias, \\ Java, \\ Borneo \\ Sikkim, \\ Assam, \\ Burma, \\ Perak}

Formosa

Bornco

Singapore, Borneo,

Pulolaut

Südindien

Burma

Assam

Perak, Bornco

Westelina

Bombay,

Nilgiri

Hills

Burma,

Cambodja

Assam,

Burma

Nordwest-

himalaya

(Chitral)

Assam

Ost- und

Nordchina,

I.'Tschusan,

Formosa,

Assam,

Burma 
Fortunei de l'Orza, Lep. Jap. p. 38 (1869). - Hamps., Cat. Lep. Phal. 1, p. 104, t. 4, f. 12 (1898). Leech, Trans. Ent. Soc. Lond. p. 319 (1898). Seitz, Grossschmett. d. Erde, T. pal. 2, p. 39, t. 9, d (1910).

ab. erebina Butl., Trans. Ent. Soc. Iond. p. 5 (1881). - Hamps., Cat. Lep. Phal. 1, p. 104 (1898). - Seitz, Grossschmett. d. Erde, F. pal. 2, p. $39(1910)$.

Fruhstorferi Rothsch., Nov. Zool. 17, p. 435 (1910) (Amata).

gelatina Hamps., Illustr. Het. Br. Hus. 8, p. 43, t. 139, f. 1 (1890); Moths Ind. 1, p. 214 (1892); Cat. Lep. Phal. 1, p. 103 (1898).

geon Druce, Proc. Zool. Soc. Iond. p. 519, t. 32, f. 2 (1885). - Hamps., Cat. Lep. Phal. 1, p. 88 (1898). - Semp., Schmett. Philipp. 2, p. 422 (1898).

germana Feld., Wien. Ent. Monatsschr, 6, p. 37 (1862). - Hamps., Cat. Lep. Phal. 1, p. 93 (1898). Seitz, Grossschmett. d. Erde, F. pal. 2, p. 40, t. $9, \mathrm{~g}(1910)$.

fenestrata Herr.-Schäff., Aussereur. Schmott. p. 72, f. 270 (1855) (nec Drury).

thelebus Mén., Schrenck's Reisen Amurld. 2, Lep. p. 48 (1859) (nee Fabr.). - Stgr., Mém. Rom. 6, p. 252 (1892).

mandar ina Butl., Journ. Linn. Soc. Lond., Zool. 12, p. 349 (1876). - Seitz, Grossschmett. d. Erdo, F. pal. 2, p. 40 , t. 9 , h (1910).

ab. nigricauda Miyake, Annot. Zool. Jap. 6, p. 161 , f. (1907).

graduata Hamps., Cat. Lep. Phal. 1, p. 67, t. 2, f. 28 (1898). - Scitz, Grossschmett. d. Erde, F. pal. 2, p. 40 , t. 9 , f. $(1910)$.

Grotei Moore, Proc. Zool. Soc. Lond. p. 245, t. 18, f. 4 (1871). - in Anderson, Researches in WestYunnan p. 928, t. 81, f. 7 (1878). - Hamps., Moths Ind. 1, p. 218 (1892). — Swinh., Trans. Ent. Soc. Lond. p. 31 (1895). - Hamps., Cat. Lep. Phal. 1, p. 92 (1898).

Huebneri (Hubncri) Boisd., Mon. Zyg. p. 127, t. 8, f. 4 (1829). - Hamps., Moths Ind. 1, p. 219 (1892); Cat. Lep. Phal. 1, p. 69 (1898). - Semp., Schmett. Philipp. 2, p. 420 (1898). - Snell., Tijdschr. v. Ent. 47, p. 54 (1904). - Turner, Proc. Linn. Soc. Now South Wales 29, p. 849 (1905).

Marsdeni Moore, Lep. East. Ind. Comp. p. 323 (1859); Proc. Zool. Soc. Lond. p. 197, t. 60, f. 3 (1859).

xanthomela Walk., Journ. Linn. Soc. Lond., Zool. 3, p. 184 (1860).

cingulata Wallengr., Wien. Ent. Monatsschr. 4, p. 39 (1860) (Naclia).

sangaris Wallengr., Wien. Ent. Monatsschr. 7, p. 139 (1863) (Buthysia).

contermina Walk., List. Lep. Ins. Br. MIus. 31, p. 78 (1864).
Japan, Corea, Central. china

Tonkin

Südindien

Mindanao

Amur- u.

Ussuri-

gebiet

Corea,

Central-u.

Westchina,

Japan,

I.Tschusan,

Annam

(M. C.

Vind.)

Japan

China

(Kiang-

Nan)

Südchina,

Yunnan,

Annam

(MI. C.

Vind.),

Burma

Bombay,

Singapore,

Sumatra,

Nias, Java,

Luzon,

Flores,

Thursday-I.

Lizard-I.,

Nord-

australien,

Qucensland 
pyrrhodera Meyr., Proc. Linn. Soc. New South Wales (2) 1, p. 777 (1886) (Hydrusa). - Turner, Proc. Roy. Soc. S. Austr. 22, p. 95, t. 5, f. 14 (1898).

ab. frustulenta Swinh., Cat. Het. Mus. Oxf. 1, p. 44 (1892). - Hamps., Cat. Lep. Phal. 1, p. 69 (1898).

hydatInn Butl., Journ. Linn. Soc. Lond., Zool. 12, p. 346 (1876). - Hamps., Moths Ind. 1, p. 210 (1592); Cat. Lep. Phal. 1, p. 106, t. 4, f. 14 (1898).

insuefa Swinh., Cat. Het. Mus. Oxf. 1, p. 41, t. 2, f. 16 (1892). - Hamps., Moths Ind. 1, p. 213 (1892); Cat. Lep. Phal. 1, p. 105 (1898).

interrupta Wileman, The Ent. 43, p. 220 (1910).

Jankowskyi Rothsch., Nov. Zool. 17, p. 434 (1910) (Amata).

khasiana Butl., Journ. Linn. Soc. Lond. Zool. 12, p. 345 (1876). - Hamps., Moths Ind. 1, p. 214 (1892); Cat. Lep. Phal. 1, p. 103, t. 4, f. 9 (1898).

killensis Hamps., Cat. Lep. Phal. 1, p. 87, t. 3, f. 11 (1898).

Leechi Rothsch., Nov. Zool. 17, p. 433 (1910) (Amata). acrospila Leech, Traus. Ent. Soc. Lond. p. 322 (1898) (nec Feld.).

leucozonn Hamps., Ann. \& Mag. Nat. Hist. (8) 8, p. 393 (1911).

leucozonoides Rothsch., Nor. Zool. 19, p. 123 (1912) (Amata).

longipew11is Walk., Journ. Linn. Soc. Lond., Zool. 6, p. 91 (1862). 一 Stwinh., Cat. Het. Mus. Oxf. t. 2, f. 13 (1892). — Hainps., Cat. Lep. Phal. 1, p. 113 (1898).

Inecrua Wileman, The Ent. 43, p. 220 (1910). ab. flava Wilemau, 'The lint. 43, p. 220 (1910).

lucina Butl., Journ. Linn. Soc. Lond., Zool. 12, p. 345 (18;6). - Hamps., Moths Ind. 1, p. 214 (1892); Cat. Lep. Phal. 1, p. 104, t. 4, f. 10 (1895).

luteifaseia Hamps., Moths Ind. 1, p. 218 (1892); Cat. Lep. Plial. 1, p. 97, t. 3, f. 25 (1898).

madureusis Hamps., Ann. \& Mag. Nat. Hist. (7) 8, p. 166 (1901): Journ. Bombay Nat. Hist. Soe. 16, p. 151, t. D, f. 27 (1904).

Masoni Moore, Proe. Zool. Soc. Lond. p. 845, t. 53, f. 4 (1878). - Hamps., Moths Ind. 1, p. 215 (1892); Cat. Iep. Plıal. 1, p. 95 (1898). - Scitz, Grossschmett. d. Erde, 1. pal. 2, p. 40 (1910).

Davidi Poujade, Bull. Soc. Ent. France (6) 4, p. CXXXVI (1885).

megista Hamps., Cat. Lep. Phal. 1, p. 107, t. 4, f. 13 (1898).

ulelaena (melas) Walk., List Lep. Ins. Br. Mus. 1, p. 133 (1854). - Butl., Illustr. Het. Br. Mus. 1, p. 17, t. 6, f. 10 (1877). - Hamps., Moths Ind. 1, p. 216 (1892); Cat. Lep. Phal. 1, p. 96 (1898). Andersoni Moore, Proc. Zool. Soc. Lond. p. 244, t. 18, f. 1 (1871); in Anderson, Researches in West Yunnan p. 926, t. 81, f. 4 (1878).
Sikkim, Bengalen, Zentralindien

Bengalen

Formosa

China

Assam

Borneo

Westchina

Borneo

"

,

Formosa

Nepal, Bhutan, Sikkim, Bengalon

Assam

Südindien

West- und Zentral-

china,

Tenasserim

Bornco

Nepal, Sikkim, Assam, Burma, Yunnan 
molanocera Hamps., Ann. \& Mag. Nat. Hist. (7) 11, p. 338 (1903).

mclanoproctis (molaproctis) Hanıs., Ann. \& Mag. Nat. Hist. (7), 8 p. 166 (1901).

midas Butl., Journ. Linn. Soc. Lond., Zool. 12, p. 344 (1876). - Swinh., Cat. Het. Mus. Oxf. 1, p. 44 (1892).

fenestrata Walk., List lep. Ins. Br. Mus. 31, p. 73 (1864). - Hamps., Cat. I.ep. Phal. 1, p. 60, t. 2, f. 14 (1898) (nec Drury 1773).

minor Warr., Proc. Zool. Soc. Lond. p. 294 (1888). Hamps., Moths Ind. 1, p. 213 (1892); Cat. Lep. Plial. 1, p. 107, t. 4, f. 15 (1898).

Muirhoadi Feld., Wien. Ent. Monatssehr. 6, p. 37 (1862). - Hamps., Cat. Lcp. Phal. 1, p. 95, t. 3, f. 13 (1898). - Leech, Trans. Ent. Soc. Lond. p. 322 (1898). - Seitz, Grossschmett. d. Erde, F. pal. 2, p. 40 , t. 9 , g (1910).

v. aucta Lecch, The Ent. 31, p. 153 (1898). Hamps., Cat. Lep. Phal. 1, p. 95 (1898). Seitz, Grossschmett. d. Erde, F. pal. 2, p. 40 (1910).

newara Moorc, Lep. Atkins. p. 12 (1879). - Hamps., Moths Ind. 1, p. 217 (1892); Cat. Lep. Plial. 1, p. 94 , t. 3 , f. 18 (1898).

ochreipuncta Hamps., Moths Ind. 1, p. 222 (1892); Cat. Lep. Phal. 1, p. 64, t. 2, f. 17 (1898).

orphana Sncll., Tijdschu. v. Ent. 47, p. 57 (1904).

Owstoni Rothsch., Nov. Zool. 18, p. 155 (1911) (Amata).

palanana Scmp., Schmett. Philipp. 2, p. 419 (1898).

pasca(-us) Lcech, Trans. Ent. Soc. Lond. p. 124, t. 9, f. 1 (18S9); il). p. 320 (1898). - Hamps., Cat. Lep. Phal. 1, p. $9 \div$ (1898). — Scitz, Grossschmett. d. Erde, 1. pal. 2, p. 40, t. 9, h (1910).

- passalis Fabr., Spcc. Ins. 2, p. 159 (1871) (Zygaena). - Boisd., Mon. Zyg. p. 120 (1829). - Moorc, Lep. Ceyl. 2, p. 35 (1882). - Hamps., Moths Ind. 1, p. 219 (1892); Cat. Lep. Phal. 1, p. 86 (1898).

cerbera Sulz., Abgek. Gosch. Ins. t. 20, f. 8 (1776) (Zygaena) (nce L.).

creusa Cram., Pap. Exot. 3, p. 94, t. 248, f. I (1780) (Sphinx).

Latreillei Boisd., Mon. Zyg. p. 117, t. 7, f. 5 (1829). ab. mantana Butl., Journ. Linn. Soc. Lond., Zool. 12, p. 349 (1S76). - Moorc, Lep. Ceyl. 2, p. 36, t. 94, f. 5 (1882). - Hamps., Cat. Lep. Phal. 1, p. $S 6$ (1898).

Biologie: Hamps., Journ. Bombay Nat. Hist. Soc. 20 , p. 95 (1910).

pectoralis Walk., List Lcp. Ins. Br. Mus. 1, p. 133 (1854). - Butl., Illustr. Het. Br. Mus. 1, p. 17, t. 6, f. 15 (1877). - Hamps., Moths Ind. 1, p. 224 (1892); Cat. Lcp. Phal. 1, p. 62 (1898).

basirufa Swinh. Cat. Het. Mus. Oxf. 1, p. 51 (1892) (Hydrusa).
Nordehina

Philippinen (Samboangan)

Cambodja

Pundjab

China

China

(Kwei-

Chow)

Sikkim,

Bliutan

Vorderindien :

Java

Ganjam

Annam,

Hainan

Luzon

Zentral- und Westchina

Südindion bis Bombay

und

Bengalen,

Assam,

Ccylon

Burma,

Cambodja,

Tenasse-

rim,

Andama-

nen 
pentazounta Hamps., Cat. Lep. Phal. 1, p. 99, t. 3, f. 27 (1898); Journ. Bombay Nat. Hist. Soc. 13, p. 50 (1900).

perixantlia Hamps., Cat. Lep. Pinal. 1, p. 97, t. 3, f. 17 (1898). - Leceh, Trans. Lnt. Soe. Lond. p. 321 (1898). - Suitz, Grossschmett. d. Erde, F. pal. 2, p. 39 , t. 9 , f $(1910)$.

v. sinensis Rothseh., Nov. Zool. 17, p. 435 (1910) (Amata).

persimilis Leeel, The Ent. 31, p. 152 (1898); Trans. Ent. Soe. Lond. p. 322 (1898). - Hamps., Cat. Lep. Phal. 1, p. 97 (1598). — Seitz, Grossschmett. d. Eirde, F. pal. 2, p. 39 (1910).

Pfeifferae Moore, Lep. Last Ind. Comp. p. 324 (1859); Proc. Zool. Soc. Lond. p. 198, t. 60, f. 5 (1859). - Hamps., Cat. Lep. Phal. 1, p. 88 (1898). Sncll., Iijdsehr. v. Ent. 47, p. 55 (1904).

a cuminata Snell. in Veth, Midden-Sumatra, Lep. p. 31 , t. 3 , f. 5 (1850).

philippinensis Hamps., Ann. \& Mag. Nat. Hist. (7) 19, p. $222(1907)$.

phoenienzoua Hanps., Journ. Bombay Nat. Hist. Soe. 11, p. 284 (1897); Cat. Lep. Phal. 1, p. 63, t. 2, f. 13 (1898).

- polymita Sparrm., Amoen. Aead. 7, p. 504, not. (1769) (Sphinx). - Hamps., Cat. Lep. Plial. 1, p. 92 (1898).

fenestrata Drury, Ill. Exot. Ent. 2, p. 50, t. 28, f. 5 (1773) (Sphinx). — Fabr., Syst. Ent. p. 552 (1775). - Boisd., Mon. Zyg. p. 124, t. 8, f. 1 (1829). - Semp., Schmott. Philipp. 2, p. 420 (1898).

thelebus Fabr., Mant. Ins. 2, p.103(1787)(Zygaena). - Boisd., Mon. Zyg. p. 122 (1829). - Auriv., Ent. 'Tidskr. 18, p. 154 (1897).

Pratti Lecel, Trans. Lnt. Soc. Lond. p. 123, t. 9, f. 3 (1889). - Hamps., Cat. Lep. Phal. 1, p. 64 (1898). - Seitz, Grosssehmett. d. Errde, F. pal. 2, p. $40(1910)$.

Pryeri Hamps., Cat. Iep. Phal. 1, p. 102, t. 4, f. 8 (1898). pseudexterisa Rothseh., Nov. Zool. 17, p. 435 (1910) (Amata).

punctala Semp., Schmett. Plilipp. 2, p. 421 (1898) (Hydrusa).

quadrifuscia Hamps., Moths Ind. 1, p. 215 (1892); Cat. Lep. Phal. 1, p. 100 , t. 4, f. 2 (1898).

quadripunctata Rothsch., Nov. Zool. 17, p. 433 (1910) (Amata).

Ribbci Röbcr (cf. p. 31).

rubrozonata Poujade, Bull. Soc. Ent. France (6) 6 , p. CXVII (1886). - Hamps., Cat. Lep. Plial. 1, p. 85 , t. 3 , f. 7 (1898). - Seitz, Grossschmett. d. Erde, F. pal. 2, p. 40 (1910).

ab. levcoma Leeeh, The Ent. 31, p. 154 (1898). - Kamps., Cat. Lep. Phal. 1, p. 85 (1898). Seitz, Grossschmett. d. Erde, F. pal. 2, p. 40 , t. $9, \mathrm{f}(1910)$.

Burma

Formosa

Westeluina$$
\text { , }
$$

Sumatra,

Java,

Palawan

Luzon,

Mindoro,

Mindanao

Andamanen

Sïdchina,

Tonkin,

Mindoro

Zentralchina

Borneo

,

Iuzon

Burma

Sumatra

Philippinen, Celebes

Westehina 
sala Swinh., Ann. \& Mag. Nat. Hist. (7) 9, p. 77 (1902). serrata Hamps., Motlis Ind. 1, p. 221 (1892); Cat. Lep. Phal. 1, p. 70 , t. 2 , f. 24 (1898).

sibulana Semp., Schmett. Philipp. 2, p. 419 (1898).

siguata Walk., List Lep. Ins. Br. Mus. 7, p. 1594 (1856). - Hamps., Cat. Lep. Phal. 1, p. 97, t. 3, f. 24 (1898).

Sladeni Moore, Proe. Zool. Soe. Lond. p. 245, t. 18, f. 5 (1871); in Anderson, Researches in West Iumnan p. 927 , t. 81, f. 8 (1878). - Hamps., Moths Ind. 1, p. 217 (1892); Cat. Lep. Phal. 1, p. 91 (1898).

Snelleni Rothsch., Nov. Zool. 17, p. 432 (1910) (Amata). sperbins Fabr., Mant. Ins. 2, p. 103 (1787) (Zygaena). - Boisd., Mlon. Zyg. p. 126 (1829). — Hamps., Cat. Lep. Phal. 1, p. 106 (1898).

Atkinsoni Moore, Proo. Zool. Suc. Lond. p. 245, t. 18, f. 2 (1871); in Anderson, Rescarehes in West Yunnan, p. 927, t. 81, f. 5 (1878). Butl., Journ. Linn. Soc. Lond., Zool. 12, p. 347 (1876). - Hamps., Mloths Ind. 1, p. 212 (1892); Cat. Lep. Phal. 1, p. 106 (1898).

stellaris Sncll., Iris 8 , p. 130 (1895). - Humps., Cat. Lep. Phal. 1, p. 112, t. 4, f. 222(1898). — Snell., Tijdsehr. v. Ent. 47, p. 57 (1904).

submarginalis Walk., Charact. Undeser. Het. p. 89 (1869). - Hamps., Mloths Ind. 1, p. 218 (1892); Cat. Lep. Phal. 1, p. 91, t. 3, f. 15 (1898).

synphona Swinh., Ann. \& Mag. Nat. Hist. (7) 19, p. 201 (1907).

taiwana Miyako, Annot. Zool. Jap. 6, p. 81 (1907).

tenuis Walk., List Lep. Ins. Br. Mns. 7, p. 1595 (1856). — Snell., Tijdschr. v. Ent. 22, p. 70, t. 6, f. 2 (1879). - IIamps., Cat. Lep. Phal. 1, p. 89 (1898). - Semp. Sehmett. Philipp. 2, p. 421 (1898). — Sncll., 'Tijdiselrr. v. Ent. 47, p. 56 (1904).

linearis Walk., List Lep. Ins. Br. Mlus. 31, p. 77 (1864). - Semp., Schmett. Philipp. 2, p. 422 (1898).

v. deflocea Swinh., Cat. Het. Mus. Oxf. p. 45 (1892). - Semp., Schmett. Philipp. 2, p. 421 (1898). - Hamps., Cat. Lep. Plıal. 1, p. 89 (1898).

tetragonaria Walk., Journ. Linn. Soc. Lond., Zool. 6, p. 87 (1862). - Hamps., Cat. Lep. Phal. 1, p. 89, t. 3 , f. $12(1898)$.

tetrazonata Hamps., Cat. Lep. Phal, 1, p. 101, t. 4, f. 4 (1898).

- thoraclea Moore, Ann. \& Mag. Nat. Hist. (4) 20, p. 343 (1877) (Phacusa).; Lep Ceyl. 2, p. 38, t. 95, f. 7 (1882). - Hamps., Moths Ind. 1, p. 224 (1892); Cat. Lep. Phal. 1, p. 63, f. 32 (1898).

Tieannis Hamps., Ann. \& Mag. Nat. Hist. (7) 19, p. 223 (1907).

tinopera (teinopera) Hamps., Cat. Lop. Phal. 1, p. 76, f. 34 (1898).

torquata(-us) Leech, Trans. Ent. Soc. Lond. p. 124, t. 9, f. 2 (1889). - Hamps., Cat. Lep. Phal. 1, p. 95

Borneo

Assam

Mindanav

Java

Burma, Yunnan

Java

Sikkim,

Bhutan,

Assam,

Yunnan,

Burma,

Tonkin,

Siam (M. C.

Vind.),

Perak

Siam, Perak,

Sumatra,

Java,

Borneo

Sikkim,

Bhutan,

Assam,

Burma

Borneo

Formosa

Java,

Celebes,

Palawan,

Plilippinen

Pliilippinen

Borneo

Formosa

Ceylon

Plilippinen:

Ticao

Borneo

Zentral- u. Westchina 
(1898). - Seitz, Grossschmett. d. Erde, F. pal. 2 , p. $40(1910)$.

unifascia Hamps., Noths Ind. 1, p. 212 (1892); Cat.

Lep. Phal. 1, p. 112, t. 4, t. 20 (1898).

Vaudepolli Rothsch., Nov. Zool, 17, p. 436 (1910) (Amata).

verecunda Swinh., Ann. \& Mag. Nat. Hist. (7) 9, p. 78 (1902).

vitren Walk., List Lep. Ins. Br. Mus. 7, p. 1594 (1856). - Hamps., Moths Ind. 1, p. 218 (1892); Cat. Lep. Phal. 1, p. 90, t. 3, f. 14 (1898).

Austeni Moore, Proc. Zool. Soc. Lond. p. 389 (1879).

polyzonata Hamps., T'rans. Ent. Soc. Lond. p. 283 (1895); Moths Ind. 4, p. 466 (1896).

Wallacei(-ii) Moore, Proc. Zool. Soc. Lond. p. 198, t. 60, f. 6 (1S59). - Hamps., Cat. Lop. Phal. 1, p. 61 (1898). - Sncll., Tijdschr. v. Ent. 47, p. 53 (1904).

Wilemani Rothsch., Nov. Zool. 18, p. 154 (1911) (Amata). Wimberleyi Swinh., Proc. Zool. Soc. Lond. p. 400, t. 43, f. 11 (1889). - Hamps., Moths Ind. 1, p. 219 (1892); Cat. Lep. Phal. 1, p. 88 (1898).

xanthoma Lecch, The Ent. 31, p. $15^{2}$ (1898). - Hamps., Cat. Lep. Phal. 1, p. 94, t. 3, f. 20 (1898). Scitz, Grossschmett. der Erdc, F. pal. 2, p. 4l, t. 9, g (1910).

yunnancusis Rothsch., Nov. Zool. 18, p. 155 (1911) (Amata).

\section{d) Australische Arten.}

adjuucta Walk., List Lep. Ins. Br. Mus. 31, p. 74 (1864). - Hamps., Cat. Lep. Phal. 1, p. 111, t. 4, f. 18 (1898).

Alberti Rothsch., Nov. Zool. 18, p. 154 (1911) (Amata).

amoenaria Swinh., Ann. \& Mag. Nat. Hist. (7) 9, p. 417 (1902).

anuulata Fabr., Syst. Ent. p. 551 (1775) (Zygaena). Boisd., Mon. Zyg. p. 122, t. 7, f. 8, t. 8, f. 2 (1829). - Meyr., Proc. Liun. Soc. New South Wales (2) 1, p. 783 (1886). - Hamps., Cat. Lep. Plial. 1, p. 72, f. 33 (1898). - 'Turner, Proc. Roy. Soc. S. Austr. 22, p. 98 (1898); Proc. Linn. Soc. Ncw Sonth Wales 29, p. 846 (1905).

ab. nigriceps Butl., Journ. Linn. Soc. Lond., Zool. 12, p. 352 (1s76) (Hydrusa). - Hamps., Cat. Lep. Phal. 1, p. 73 (1898).

ab. intensa Butl., Journ. Linn. Soc. Lond., Zool. 12, p. 353 (1876) (Hydrisa). - Mcyr., Proc. Liun. Soc. New South IVales (2) 1, p. 784 (1886). - Hamps., Cat. Lep. Phal. 1, p. 73 (1898).

antithefa Meyr., Proc. Linn. Soc. New Nouth Wales (2) 1, p. 779 (1886) (Mydrusa). - Hamps., Cat. Isep. Phal. 1, p. 74 (1898). - Turner, Proc. Linn. Soc. New South Wales 29, p. S4S (1905).

ab. anepsia Mcyr., Proc. Linn. Soc. New South Wales (2) 1, p. 779, (1886) (Hydrusa). - Hamps., Cat. Lcp. Phal. 1, p. 73 (1898). - Turner, Proc. Linn. Soo. New South Wales 29, p. 848 (1905).

Sikkim

Sumatra

Assam

Assam,

Burma

Malakka (M.

C. Vind.),

Java

Formosa

Andamanen

Westchina

Yunnan

Amboina,

Ceram,

Key-I.

Brit.-

Neuguinea

West-

australien

Philippinen,

Neuguinea,

Queens.

land,

Neusiid-

wales,

Victoria,

Tasmanien

Queenslanr? 
aperta Walk., List Lop. Ins. Br. Mrus. 31, p. 72 (1864). - Hamps., Cat. Lep. Phal. 1, p. 71 (1898). Turner, Proc. Linn. Soc. New South Walcs 29, p. $\$ 53$ (1905).

? polidamon Cram., Pap. Exot. 3, p. 75, t. 237, f. B (1780) (Sphin $x$ ). - Boisd., Mon. Zyg. p. 125, t. 8 , f. 3 (1529) (nomen prius, sed ineertum).

ab. nesothetis Meyr., Proc. Linn. Soc. Now South Wales (2) 1, p. 783 (18S6) (Hydrusa). - Hamps., Cat. Lep. Pnal. 1, p. 72 (1898).

arfakensis Rothsch., Nov. Zool. 18, p. 545 (1911) (Amata).

attenuata Hamps., Ann. \& Mag. Nat. Hist. (7) 8, p. 167 (1901). - Turner, Proc. Linn. Soc. New South Wales 29 , p. 850 (1905).

aurofasciatn Swiul., Cat. Ilet. Mus. Oxf. 1, p. 43, t. 2 , f. 19 (1892). - Hamps., Cat. Lep. Plial. 1, p. 85 (1898).

basigera Walk., List Lep. Ins. Br. Mus. 31, p. 77 (1864). - Hamps., Cat. Lep. Phal. 1, p. 96, t. 3 , f. 23 (1898).

v. gilolensis Rothsch., Nov. Zool. 17, p. 434 (1910) (Amata).

bicolor Walk., List Lep. Ins. Br. Mus. 1, p. 255 (1854) (Euchromia). - Butl., Illustr. Het. Br. Mus. 1, p. 19, t. 9, f. 1 (1877). - Meyr., Proc. Linn. Suc. New South Wales (2) 1, p. 785 (1886). Hamps., Cat. Lep. Plaal. 1, p. 75 (1898). - Turner, Proo. Linn. Soc. New South Wales 29, p. 844 (1905).

celehesa Walk., List Lep. Ins. Br. Mus. 31, p. 70 (1864). - Hamps., Cat. Lop. Phal. 1, p. 85 , t. 3 , f. 8 (I898).

gracilis Röber, Iris 1, p. 30 , t. 1 , f. 7 (1885).

clvira Pagenst., Ent. Nachr. 22, p. 51 (1896) (Zygaena).

ehlorometis Mcyr., Proc. Linn. Soc. New South Wales (2) 1, p. 782 (1886) (Hydrusa). - Hamps., Cat. Lep. Phal. 1, p. 6S, t. 2, f. 22 (1898). - Turner, Proo. Roy. Soc. S. Austr. 22, p. 95 (1898); Proc. Linn. Soc. New Sonth Wales 29, p. 840 (1905).

choneutospila Turner, Proe. Linn. Soe. Now Sonth Wales 29 , p. 844 (1905).

clıroma Swinh., Cat. Het. Mhus. Oxf. 1, p. 50 (1892) ( $\mathrm{H}_{y}$ drusa). - Flamps., Cat. Lep. Phal. 1, p. 73 (1898). - Swinh., Ann. \& Mag. Nat. Hist. (7) 10, p. 49 (1902). - Turner, Proc. Linn. Soc. New South Wales 29, p. 849 (1905).

Clementsi Hamps., Ann. \& Mag. Nat. Hist. (7) 8, p. 167 (1901).

eliromatica Turner, Proc. Linn. Soc. New South Wales 29, p. 845 (1905).

cyannura Moyr., Proc. Linn. Soc. New Sonth Wales (2) 1, p. 778 (1886) (Hydrusa). - Hamps., Cat. Lep. Phal. 1, p. 75 (1898). - Turner, Proc. Linn. Soc. New South Wales 29, p. 847 (1905).

Neuguinea(?), Neusüdwales

Holl.-

Neuguinea

Nord-

australien

(Hoywood-

Insel,

Bathurst-I.,

Queens-I.)

Neuguinea

(ubi?)

Batjan

Halmaheira

Qucensland

Celebes

Queensland, Nousüdwales

Quecnsland

Nordwestaustralien

Queensland

Thursday-I. 
dysehluena Turner, Proc. Linn. Soc. New South Wales 29, р. 855 (1905).

Fveretti Rothsch., Nov. Zool. 17, p. 436 (1910) (Amata). Henrici Rothscl., Nov. Zool. 17, p. 437 (1910) (Amata).

leptaspila Turner, Proc. Linn. Soc. New South Wales 29 , p. 852 (1905).

Huebneri Boisd. (cf. p. 22).

humeralis Butl., Journ. Linn. Soc. Lond., Zoul. 12, p. 352 (1876) (Hydrusa). - Hamps, Cat. Lep. Phal. 1, p. 63, t. 2, f. 15 (1898). - Turner, Proc. Roy. Suc. S. Austr. 22, p. 97 (1898); Proc. Linn. Soc. New South Wales 29, p. 850 (1905).

olinda Swinh., Cat. Het. Mus. Oxf. 1, p. 53 (1892) (Eressa). - Hamps., Cat. Lcp. Piral. 1, p. 72 (1898).

lyalota Meyt., Proc. Linn. Soc. New South Wales (2) I, p. 777 (1886) (IIydrusa). - Hanıps., Cat. Lep. Plial. 1, p. 69, (1898). - Turner, Proc. Linn. Soc. New South Wales 29, p. 848 (1905).

insularis Butl., Journ. Linn. Soc. Lond., Zool. 12, p. 353 (1876) (Hydrusa). - Hamps., Cat. Lep. Phal. 1, p. 73, t. 2, f. 20 (1898). - Turner, Proc. Linn. Soc. Now South Wales 29, p. 841, (1905).

stelotis Mcyr., Proc. Linn. Soc. New South Wales (2) 1, p. 776 (1886) (Hydrusa).

kalidupensis Rotliscli., Nov. Zool. 17, p. 432 (1910) (Amata).

lampetis Tumer, Proc. Roy. Soc. S. Austr. 22, p. 94, t. 5, f. 12 (1898) (Hydrusa); Proc. Linn. Soc. New South Wales 29 , p. 842 (1905).

lencaema Meyr., Proc. Linn. Soc. New South Wales (2) 1, p. 778 (1886) (Hydrusa). - Hamps., Cat. Lep. Phal. 1, p. 74 (1898). - Thimer, Proc. Roy. Soc. S. Austr. 22, p. 95, t. 5, f. 9 (1898); Proc. Linn. Soc. New South Wales 29, p. 851 (1905).

lueta Lucas, Proc. Soc. Quecnsland 16, p. 73 (1901).

nagistri Turner, Proc. Linn. Soc. New South Wales 29, p. 846 (1905).

a perta Meyr., Proc. Iinn. Soc. Now Soutlı Wales (2) 1, p. 783 (1886) (Hydrusa) (ncc WWlk.).

marella But!., Journ. Linn. Soc. Lond., Zool. 12, p. 350 (1876). - Hamps., Cat. Lep. Phal. 1, p. 70, t. 2, f. 26 (1898). - Turner, Proe. Linn. Soc. New South Wales 29, p. 842 (1905).

ecliptis Meyr., Proc. Linn. Soc. New South Wales (2) 1, p. 776 (1886) (Hydrusa). - Turner, Proc. Roy. Soc. S. Austr. 22, p. 94, t. 5, f. 11 (1898).
Nordqueensland (Magnetic-1.)

Flores

Dammer,

Letti,

Scrnatta,

Timorlaut,

Larat

Qucensland

Bombay bis

Java und

Luzon.

Flores,

Thursday-I.,

Lizard-I,.

Nordaustral.

Qucensland

Nord-

australicn,

Quecnsland

Cap York

Nord-

australien

(Barnard-I.),

Queensland

I. Toekan Besi

b. Culebes

Quecnsland

,

Neusüidwales

Qucensland 
melitospila Turner, Proc. Linn. Soc. New South Wales 29, p. 853 (1905).

metaphaea Hamps., Cat. Lep. Phal. 1, p. 68, t. 2, f. 27 (1898).

Queensland

Brit.-

Neuguinca

ochreopunctata Pagenst., Lep. Bismarck-Archip. 2, p. $24(1900)$.

orphnaea Turner, Proc. Roy. Soc. S. Austr. 22, p. 98 (1898) (Hydrusa): Proc. Linn. Soc. New South Wales 29, p. 843 (1905).

pactolina Walk., List Lep. Ins. Br. Mus. 31, p. 76 (1864). - Hamps., Cat. Lep. Phal. 1, p. 71, t. 2, f. 19 (1898). - Turner, Proe. Linn. Soc. New South Wales 29, p. 841 (1905).

sphenophora Turner, Proc. Roy. Soc. S. Austr. 22, p. 94.(1898) (Hydrusa).

paradelpha Turner, Proc. Linn. Soc. New Sonth Wales 29, p. $846(1905)$.

paraula Meyr., Proc. Linn. Soc. New South Wales (2) 1, p. 779 (1886) (Hydrusa). - Turner, ib. 29, p. 851 (1905).

macroplaca Meyr., Proc. Linn. Soc. Now South Walcs (2) 1, p. 781 (I886) (Hydrusa). - Hamps., Cat. Lep. Phal. 1, 1. 74, t. 3, f. 1 (1898).

phaeochyta Turner, Proc. Linn. Soc. New South Wales 31, p. 678 (1907).

phepsalotis Meyr., Proc. Linn. Soc. New South Wales (2) 1, p. 781 (1886) (Hydrusa). - Hamps., Cat. Lep. Phal. 1, p. 73 (1898). - Turner, Proc. Roy. Soc. S. Austr. 22, p. 99, t. 5, f. 13 (1898); Proc. Linn. Soc. New South Wales 29, p. 845 (1905).

ab. eschatias Meyr., Proc. Linn. Soc. New South Wales (2) 1, p. 785 (1886) (Hydrusa). - Hamps., Cat. Lep. Phal. 1, p. 75 (1898). - Turner, Proc. Linn. Soc. New South Wales 29, p. 845 (1905). prosomoea Turner, Proc. Linn. Soc. New South Wales 29, p. $850(1905)$.

pyrocoma Meyr., Proc. Linn. Soc. New South Wales (2) 1, p. 780 (1886) (Hydrusa). - Hamps., Cat. Lep. Phal. 1, p. 73 (1898). - Turner, Proc. Linn. Soc. New South Walcs 29, p. $854(1905)$.

cingula ta Butl, Journ. Linn. Soc. Lond., Zool. 12, p. 352 (1876) (Hydrusa) (nec Weber 1801). Hamps., Cat. Lep. Phal. 1, p. 73 (1898).

a perta Turner, Proc. Roy. Soc. S. Austr. 22, p. 95, t. 5, f. 1-6, 8 (1898) (Hydrusa) (nec TValk.).

synedra Meyr., Proc. Linn. Soc. New South Wales (2) 1, p. 780 (1886) (Hydrusa).

hesperitis Meyr., Proc. Linn. Soc. New South Wales (2) 1, p. 781 (1886) (Hydrusa).

mochlotis Mey1:, Proc. Linn. Soc. New South

Wales (2) 1, p. 782 (1886) (Hydrusa). - Pagenst., Denkschr. med.-nat. Ges. Jena 8, p. 212, t. 13, f. 3 (1895).

Biologie: Turner, Proc. Linn. Soc. New South Wales 29 , p. 854 (1905).

recedens Lucas, Proc. Linn. Soc. New South Wales (2) 6, p. 281 (1891) (Hydrusa). - Hamps., Cat. Lep.

Queensland

Neuguinea (ubi?), Qucensland, Neusüdwales, ? Südaustralien

Queensland 
Phal. 1, p. 72 (1898). - Turncr, Proc. Roy. Soc. S. Austr. 22, p. 99 (1898): Proc. Linn. Soc. New South TVales 29, p. 844 (1905).

Rlbbei Röber, Iris 1, p. 29, t. 1, f. 6 (1885). - Hamps., Cat. Lep. Phal. 1, p. 102 , t. 4, f. 6 (1898).

polusca Druce, Ann. \& Mag. Nat. Hist. (6) 15, p. 43 (1S95).

similluıa Rothsch., Nov. Zool. 17, p. 434 (1910) (Amata).

stenozona Hamps., Cat. Lep. Phal. 1, p. 69, t. 2, f. 21 (1898). - Tumer, Proc. Linn. Soe. New South Wales 29, p. 842 (1905).

sulana Lathy, The Ent. 32, p. 116 (1899).

tenuis Walk. (cf. p. 26).

trifenestra Röber, Tijdsclır. v. Ent. 34, p. $3: 24$ (1891); 35 , t. 6, f. 2 (1892). - Hamps., Cat. Lep. Phal. 1, p. 81,537 (1898).

trigonophora Turner, Proc. Roy. Soc. S. Austr. 22, p. 97, t. 5, f. 7 (1898) (Hydrusa); Proc. Linn. Soc. New South Wales 29, p. 853 (1905).

Tumneyi Rothsch., Nov. Zool. 17, p. 43 (1910) (Amata).

wuka Pagenst., Jahrb. Nass. Ver. Naturk. 39, p. 119 (1886). - Hamps., Cat. Lep. Phal. 1, p.65, t. 2, f. 16 (1898).

v. Tianda Hamps., Cat. Lep. Phal. 1, p. 65 (1898). ranthosoma Turner, Proc, Roy. Soc. S. Austr. 22, p. 93

(1898) (Hydrusa); Proc. Linn. Soc. New South Wales 29, p. 840 (1905).

cremnotherma Lower, Proc. Linn. Soc. New South Wales 25, p. 29 (1900).

xanthura Turner, Proc. Linn. Soc. New South Wales 29, Victoria 241

Philippinen, Celebes

I. Pulo Bisa b. Obi

Timor, ? Qucens. land

Sula-I.

Java,Celebes, Philippinen

Letti

Qucensland, Neusüdwales

Westaustralien

Key-I.

Trobriand-I.

Nordwestund Siudaustralien

\section{Paralaethia Hamps.}

Hamps., Ann. \& Mag. Nat. Hist. (7) 19, p. 224 (1907). subformiciua Beth.-Bak., Nov. Zool. 11, p. 382 (1904) Brit.1 (Ceryx).

Neuguinea

\section{Neëressa Hamps.}

Hamps., Ann. \& Mag. Nat. Hist. (7) 15, p. 426 (1905).

sagada Semp., Schmett. Philipp. 2, p. 423 (1898) (Tri- Luzon chaeta).

Whitehendi Rothsch., Nov. Zool. 17, p. 439 (1910). 2

\section{Exessa Walk.}

Walk., List Lep. Ins. Br. Mus. 1, p. 149 (1854). - Moore, Lep. Ceyl. 2, p. 36 (1882). - Hamps., Cat. Lep. Phal. 1, p. 115 (1898); Journ. Bombay 
Nat. Hist. Soc. 13, p. 223 (1900). - Piep. \& Sncll., Tijdschr. v. Ent. 47, p. 58 (1904). - Turner, Proc. Linn. Soc. New South Wales 29, p. 855 (1905). ? Ctenandra Feld., Sitzungsber. Akad. Wien 63, p. 38 (1861).

Trianeura Butl., Journ. Linn. Soc. Lond., Zool. 12, p. 353, t. 28, f. 3 (1876).

Chloromeles Meyr., Proc. Linn. Soc. New South Wales (2) 1, p. 785 (1886).

\section{a) Äthiopischo Art.}

pleurosticta Hamps., Proc. Zool. Soc. Lond. p. 390, t. 36, Cungo f. $4(1910)$.

\section{b) Orientalische Arten.}

affinis Moore, Proc. Zool. Soc. Lond. p. 596, t. 59, f. 3 (1877). - Hamps., Moths Ind. 1, p. 223 (1892); Cat. Lep. Phal. 1, p. 117 (1898).

politula Swinh., Cat. Het. Mus. Oxf. 1, p. 52 (1892). annosa TValk., Journ. Linn. Soc. Lond., Zool. 3, p. 183 (1859) (Syntomis). - Swinh., Cat. Het. Mus. Oxf. t. 2, f. 17 (1892). - Hamps., Moths Ind. 4, p. 466 (1896); Cat. Lep. Phal. 1, p. 120 (1898). - Sncll., Tijdschr. v. Ent. 47, p. 59 (1904).

marcescens Feld., Reisc Nov., Lep. 2, p. 9, t. 102, f. 12 (1869) (Syntomis).

lasara Pagonst., Jalurb. Nass. Ver. Naturk. 38, p. 13, t. 2, f. 8 (18S5) (Syntomis). - Snoll., Iris 8, p. 130 (1895).

จ. Ericssoni Rothsclı., Nor. Zool. 19, p. 124 (1912). aperiens Walk., List Iep. Ins. Br. Mus. 31, p. 68 (1864) (Syntomis). - Hamps., Moths Ind. 1, p. 221 (1892); Cat. Lcp. Phal. 1, p. 119 (1898).

Moorei Butl., Journ. Linn. Soc. Lond. 12, p. 354 (1876) (Trianeura).

ab. lydia Swinh., Trans. Ent. Soc. Lond. p. 134, t. 8, f. 7 (1891) (Syntomis). - Hawps., Cat. Lep. Phal. 1, p. 120 (1898).

v. khasiana Rothsch., Nov. Zool. 17, p. 438 (1910). confinis Walk., List Lep. Ins. Br. Mus. 1, p. 149 (1854) (Glaucopis). - Moorc, Lep. Ceyl. 2, p. 36, t. 95, f. 6 (1882). - Hamps., Moths Ind. p. 223 (1892); Cat. Lep. Phal. 1, p. 116, f. 38 (1898); Journ. Bombay Nat. Hist. Soc. 13, p. 223, fig. (1900) al). musa Stwinh., Proc. Zool. Soc. Lond. p. 290, t. 20, f. 1 (1885). - Hamps., Motlis Ind. 1, p. 222 (1892); Cat. Lcp. Phal. 1, p. 116 (1898). catoria Swinh., Ann. \& Mag. Nat. Hist. (7) 6, p. 305 (1900).

confinis ab. 2 Hamps., Cat. Lep. Phal. 1, p. 116 (1898).

v. malaccensis Rothsch., Nov. Zool. 17, p. 347 (1910).

r. intensa Rothsch., Nov. Zool. 17, p. 438 (1010).

dlscinota Moorc, Lep. Atkins. p. 13 (1879) (Syntomis). - Hamps., Moths Ind. 1, p. 216 (1892); Cat. Lep. Phal. 1, p. 123, f. 44 (1898).

Andamanen

Tenasserim,

Malacca,

Singapore,

Sumatra,

Nias, Java

Sumatra

Bombay,

Blututan,

Nilgiri

Hills,

Ceylon (M.

C. Vind.)

Assam

Vorderindien,

Assam,

Burma,

Ceylon

Tonkin,

Malakka,

Mergui-I.

(M.C.Vind.)

Südindien

(Belgaum)

Assam, Tenasse-

rim, Perak 
eressoldes Hamps., Moths Ind. 1, p. 220 (1892) (Syntomis ); Cat. Lep. Phal. 1, p. 118, t. 4, f. 26 (1898). erythrosoina Hamps., Moths Ind. 1, p. 223 (1892) (Syntomis); Cat. Lcp. Phal. 1, p. 119 , t. 5, f. 23 (1898).

Everetti Rothsch., Nov. Zool. 17, p. 439 (1910).

ichneumoniformis Rothsch., Nov. Zool. 17, p. 438 (1910). lepcha Ioore, Lep. Atkins. p. 12 (1879) (Syntomis). Hamps., Moths Ind. 1, p. 217 (1892); Cat. Lep. Phal. 1, p. 121 , t. 5, f. 7 (1898).

lutulenta Snell., Tidjschr. v. Ent. 22, p. 71, t. 6, f. 4 (1879) (Coenochromia). - Hamps., Cat. Lep. Phal. 1, p. 117 (1898). - Snell., Tijdschr. v. Ent. 47, p. 58 (1904).

microchila(-us) Hamps., Moths Ind. 1, p. 212 (1892) (Syntomoides); Cat. Lep. Phal. 1, p. 119, t. 5, f. 5 (1898).

plumalis Hamps., Moths. Ind. 4, p. 466 (1896) (Syntomis).

multigutta Walk., List Lep. Ins. Br. Mus. 1, p. 134 (1854) (Syntomis). - Butl., Illustr. Het. Br. Mus. 1, p. 19 , t. 7, f. 3 (1877). - Hamps., Moths Ind. 1, p. 220 (1892); Cat. Lep. Phal. 1, p. 120, f. 39 (1898). - Leeoh, Trans. Ent. Soc. Lond. p. 323 (1898).

Blanchardi Poujade, Bull. Soc. Ent. France p. CXXXVI (1884) (Syntomis). - Hamps., Moths Ind. 1, p. 221 (1892). - Leech, Trans. Ent. Soc. Lond. p. 323 (1898).

nigra Hamps., Moths Ind. 1, p. 221 (1892) (Syntomis): Cat. Lep. Phal. 1, p. 121 , t. 5, f. 8 (1898).

quinqueeincta Hamps., Cat. Lep. Phal. 1, p. 119, t. 5, f. 3 (1898).

semifusca Hamps., Cat. Lep. Phal. 1, p. 118, t. 4, f. 28 (1898).

? sexpuncta Rothsch., Nov. Zool. 19, p. 124 (1912).

siamica Walk., List Lep. Ins. Br. Mus. 31, p. 70 (1864)

(Syntomis). - Hamps., Cat. Lep. Phal. 1, p. 123, f. 43 (1898)

simplex Ruthsch., Nov. Zool. 17, p. 439 (1910).

subaurata Walk., List Lep. Ins. Br. Mus. 1, p. 149 (1854) (Glaucopis). - Moore, Lep. Ceyl. 2, p. 36 t. 95 , f. 4 (1882). - Hamps., Moths Ind. 1, p. 223 (1892); Cat. Lep. Phal. 1, p. 117 (1898). syntomoides Rothsch., Nov. Zool. 19, p. 123 (1912). v. Hosei Rothsch., Nov. Zool. 19, p. 124 (1912). vespa Hamps., Cat. Lep. Pnal. 1, p. 118, t. 4, f. 27 (1898); Journ. Bombay Nat. Hist. Soc. 13, p. 224 (1900). vespina Rothsch., Nov. Zool. 19, p. 124 (1912).

vespoides Ruthsch., Nov. Zool. 17, p. 438 (1910).

\section{c) Australische Arten.}

angustipenna Lucas, Proc. Linn. Soc. New South Wales (2).4, p. 1087 (1890) (Hydrusa). - Hamps.,
Burma

Borneo, Natuna-I.

Assam

Sikkim

Java,

Celebes

Burma,

Lombok

Westchina,

Nepal,

Sikkim,

Burma

Westhima-

laya

(Simla)

Perak

Perak.

I. Labuan

b. Borneo

Borneo

Siam

Westhimalaya (Simla)

Ceylon, Siam (M.C.Vind.)

Borneo

Burma

Borneo

Assam

Queensland 
Cat. Lep. Phal. 1, p. 122, f. 42 (1898). - Turner, Proc. Roy. Soc. S. Austr. 22, p. 92 (1898); Proc. Linn. Soc. New South Wales 29, p. 857 (1905). Dohertyi Rotsch., Nov. Zool. 17, p. 437 (1910). furva Hamps., Cat. Lep. Phal. 1, p. 115, f. 37 (1898). - Turner, Proc. Linn. Soc. New South Wales 29 , p. 856 (1905).

goographica Meyr., Proc. Linn. Soc. Netw South Wales (2) 1, p. 785 (1886) (Chloromeles). - - Hamps., Cat. Lep. Phal. 1, 1) 118, ث. 4, l. 25 (1898). Turner, Proc. Linn. Soc. New South Wales 29, p. 856 (1905).

detola Swinh., Cat. Het. Mus. Oxf. 1, p. 53 (1892). lutulenta Sncll. (cf. p. 33).

megatorna Hamps., Cat. Lep. Phal. 1, p. 122, f. 41 (1898). - Thurner, Proc. Linn. Soc. New South Wales 29, p. 857 (1905).

microchila Hamps. (cf. p. 33).

? naclioides Fold., Sitzungsber. Akad. Wien 63, p. “"39 (1861) (Ctenandra). - Hamps., Cat. Lep. Phal. 1, p. 116 (1898) (huius gencris?).

strepsimeris Meyr., Proc. Linn. Soc. New South Wales

(2) 1, p. 786 (1886) (Chloromeles). - Turner, ib. 29, p. 856 (1905).

xanthostacta Hamps., Ann. \& Mag. Nat. Hist. (7) 11, p. 339 (1903).

ypleta Swinh., Cat. Het. Mus. Oxf. 1, p. 47 (1892) (Syntomis). - Hamps., Cat. Lep. Phal. 1, p. 121 , 33 f. 40 (1898).

Pseudoceryx Rothsch.

Rothsch., Nov. Zool. 17; p. 440 (1910).

Dohertyi Rothsch., Nov. Zool. 17, p. 440 (1910).

1

Hyalaethen Butl.

Butl., Ann. \& Mag. Nat. Hist. (5) 19, p. 216 (1887). - Hamps., Cat. Lep. Phal. 1, p. 123 (1898).

Alberti Rothsch., Nov. Zool. 17, p. 440 (1910).

bivitreata Hamps., Ann. \& Mag. Nat. Hist. (8) 4, p. 344 (1909).

decipiens Rothsch., Nov. Zool. 17, p. 440 (1910).

Dohertyi Rothsch., Nov. Zool. 17, p. 440 (1910).

Kuehni Rothsch., Nov. Zool. 17, p. 439 (1910).

Meeki Rothsch., Nov. Zool. 17, p. 439 (1910).
Adonara

Dammer, Buru.

Qucensland, Neusüdwales

Java, Celebes Queensland

Burma,

Tombok

Amboina

Nordaustralien, Queensland

Waigeu

Holl.-

Neuguinea

(I. Biak)
Salomonen: Vella-lavella

Timorlaut

Salomonen: Neugeorgia Holl.Neuguinea

I. Larat bei Timorlaut

Salomonen: Guadalcanar 
metaphaea Druce, Ann. \& Mag. Nat. Hist. (7) 1, p. 147 (1898). - Hamps., Cat. Lep. Phal. 1, p. 124, t. 5, f. 22 (1898).

v. georgiensis Rothsch., Nor. Zool. 17, p. 440 (1910).

subluten Beth.-Beker, Nov. Zool. 15, p. 180 (1908).

Woodfordi Butl., Ann. \& Mag. Nat. Hist. (5) 19, p. 216 (1887). - Hamps., Cat. Lep. Phal. 1, p. 124, 9

f. 45 (1898).

Salomonen:

Guadal-

canar

Salomonen:

Neugeorgia

Brit.-

Neuguinea

Halmaheira,

Salomonen:

Alu

\section{Epitoxis Wallengr.}

Wallengr., Wien. Ent. Monatsschr. 7, p. 138 (1863). - Svenska, Akad. Handl. (4) 5, p. 11 (1865). - Hamps., Cat. Lep. Phal. 1, p. 124 (1898).

albieincta Hamps., Ann. \& Mag. Nat. Hist. (7) 11, p. 339 (1903); Trans. Zool. Soc. Lond. 19, t.4, f. 12 (1909). amazonula (amazoula) Boisd., Voy. Delegorgue 2, p. 597 (1847) (Thyreles). - Hcrr.-Schäff., Ausscrcurop. Schmett. p. 72 , f. 272 (1855). - Wallengr., Srenska Akad. Handl. (4) 5, p. 11 (1865). Hamps., Cat. Lep. Phal. 1, p. 125, f. 46 (1898). Ansorgei Rothsch., Nov. Zool. 17, p. 441 (1910).

nigra Hamps., Ann. \& Mag. Nat. Hist. (7) 11, p. 339 (1903).

procridia Hamps., Cat. Lep. Phal. 1, p. 125, t. 5, f. 29 5 (1898).

\section{Stictonaclia Hamps.}

Hamps., Cat. Lep. Phal. 1, p. 125 (1898).

amplifieata Saaln., Ber. Senckenb. naturf. Ges. p. 261 (1879-80) (Naclia); Lep. Madag. 11, p. 146, t. 6, f. 95 (1884). - Hamps., Cat. Lep. Phal. 1, p. 125, f. 47 (1898).

anastasia Oberth., Et. Ent. 18, p. 2, t. 1, f. 8 (1893) (Naclia). - Hamps., Cat. Lep. Phal. 1, p. 126, f. 48 (1898).

? maria Oberth., Et. Lép. comp. 3 , p. 94, t. 10, f. 6,7 (1909) (Naclia) (huins generis ?).

8 marietta Oberth., Et. Lép. comp. 3, p. 95 , t. 10, f. 8 (1909) (Naclia) (huins gencris?).

myodes Guér., Icon. Regne Animal, Ins. p. 500, t. 84 bis, f. 6 (1893 (Syntomis). - Boisd., Faune Madag. p. 80 , t. 11 , f. 5 (1833). - Hamps., Cat. Lep. Phal. 1, p. 126 (1898).

zygaenoides Mab., C. R. Soc. Ent. Be]g. 28, p. CLXXXVUI (1884) (Symtomis).

agnes Oberth., Et. Ent. 18, p. 4, t. 1, f. 13 (1893) (Naclia).
Brit.-

Ostafrika

Natal

Brit.Ostafrika

Portug.Ostafrika: Gazaland Brit.-

Ostafrika 
reducta Mab., Bull. Soc. Zool. France 3, p. 84 (1878)

Madagaskar (Syntomis). - Hamps., Cat. Lep. Phal. 1, p. 127, t. 5 , f. 24 (1898).

anapera Mab., Bull. Soc. Zool. France 3, p. 85

6 (1878) (Syntomis).

\section{Pseudonaclia Butl.}

Butl., Journ. Linn. Soc. Tond., Zool. 12, p. 351 (1865). - Hamps., Cat. Lep. Phal. 1, p. 127 (1898).

bifasciata Auriv., Sjöstedt Exped. Kilim., Lep. p. 24, Deutscht. 1, f. 10 (1909).

puella Boisd., Voy. Delegorgue 2, p. 596 (1847) (Naclia). - Wallengr., Svenska Akad. Handl. 4 (5), p. 13 (1865). - Hamps, Cat. Lep. Phal. 1, p. 128 , f. 49 (1898).

v. minor Rothsch., Nov. Zool. 17, p. 441 (1910).

2

Natal,

Delagoa-

bai, Mashonaland,

Brit.Rhodesia

Ostafrika

\section{Mieronaclia Hamps.}

Hamps., Cat. Lep. Phal. 1, p. 128 (1898).

bicolor Rothsch., Nov. Zool. 18, p. 155 (1911).

leopardina Ruthsch., Nov. Zool. 18, p. 155 (1911).

muscella Mab., C. R. Soe. Ent. Belg. 28, p. CLXXXVIII,

(1884) (Syntomis). - Hamps., Cat. Lep. Phal. 1, p. 128 (1898). - ? Auriv. in Voeltzkow, Reise Ostafrika 2, p. 311 (1909).

flavia Oberth., Et. Ent. 18, p. 5, t. 1, f. 1, 2 (1893) (Naclia).

Oberthueri Rothsch., Nor. Zool. 17, p. 155 (1911).

simplex Butl., Ann. \& Mag. Nat. Hist. (5) 4, p. 236 (1879) (Pseudonaclia). - Hamps., Cat. Lep. Phal. 1, p. 129 , f. 50 (1898).

itenera Mab., Bull. Soc. Zool. France (3) 1, p. 85 (1878) (Naclia). - Hamps., Cat. Lep. Phal. 1, p. 120, 6 t. 5, f. 14 (1898) (huius generis?).

\section{Thyrosticta Hamps.}

Hamps., Cat. Lep. Phal. 1, p. 129 (1898).

? agatha Oberth., Et. Ent. 18, p. 5, t. 1, f. 12 (1893) (Naclia). - Hamps., Cat. Lep. Phal. 1, p. 131 (1898) (huius generis?).

Butleri Mab., Le Nat. 2, p. 134 (1884) (Syntomis). Hamps., Cat. Lep. Phal. 1, p. 130, f. 51 (1898). distincta Saalm., Lep. Madag. 1, p. 144 (1884) (Dysauxes).

extensa Saaim., Lep. Madag. 1, p. 145 (1884)

Madagaskar

",

,

$"$

(Dysauxes).

Madagaskar 
ab. expallescens Saalm., Lep. Madag. 1, p. 145 (1884) (Dysauxes). - Hamps., Cat. Lep. Phal. 1, p. 130 (1898).

contigua Saalm., Lep. Madag. 1, p. 143, t. 6, f. 93 (1884) (Dysauxes). - Hamps., Cat. Lep. Phal. 1, p. 129 (1898).

? luctuosa Oberth., Ann. Soc. Ent. France 79, p. 467, t. 16, f. 4 (1911) (Naclia) (huius generis?).

minuta Boisd., Faune Vadag. p. 80 , t. 11, f. 6 (1833) (Syntomis). - Hamps., Cat. Lep. Phal. 1, p. 132 (1898). - Auris. in Voeltzkov, Reise Ostafrika 2, p. 341 (1909).

? moerens Oberth., Ann. Soc. Ent. France 79, p. 467, t. 16, f. 3 (1911) (Naclia) (huius generis?).

quadrimacula Yab., Bull. Soc. Zool. France 3, p. 85 (April 1878) (Naclia). - Hamps., Cat. Lep. Phal. 1, p. 131, f. 52 (1898).

sylvicolens Butl., Ann. \& Mag. Nat. Hist. (5) 2, p. 293, (Oct. 1878) (Pseudonaclia).

perpetua Oberth., Et. Ent. 18, p. 3, t. 1, f. 6 (1893) (Naclia).

ab. confluens Oberth., Et. Ent. 18, p. 3, t. 1, f. 10 (1893) (Naclia). - Hamps., Cat. Lep. Phal. 1, p. 131 (1898).

ab. holoxantha Zemy, nom. nov.

quadrimacula Oberth., Et. Ent. 18, p. 2, t. 1, f. 9 (1893) (Naclia) (necf. typ. Mab.). - Hamps., Cat. Lep. Phal. 1, p. 131 (1898).

Tollini Keferst., Jahrb. Akad. Erfurt (2) 6, p. 13, f. 3 1870 (Glaucopis). - Hamps., Cat. Lep. Phal. 1 , p. 132 (1898).

ab. lugens Oberth. Et. Ent. 18, p. 7, t. 1, f. 14 (1893) (Naclia). - Hamps., Cat. Lep. Phal. 1, p. 132 (1898).

trimacula Mab., Bull. Soc. Zool. France 3, p. 85 (1878) (Naclia). - Oberth., Et. Ent. 18, p. 4, t. 1, f. 11 (1893). - Hamps., Cat. Lcp. Phal. 1, p. 131 9 (1898).

Madagaskar

"

,

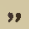

\section{Dysauxes Hübn.}

Hübn., Verz. bek. Schmett. p. 171 (1826). - Hamps., Cat. I.ep. Phal. 1, p. 132 (1898). - Spul., Schmett. Eur. 2, p. 127 (196 6). - Bergc-Rebel, Schmetterlingsb. p. 424 (1910). - Seitz, Grossschmett. d. Erde, F. pal. 2, p. 41 (1910).

Naclia Boisd., Ind. Meth. p. 60 (1840). - Hcrr.-Schäff., Syst. Bearb. Schmett. Eur. 2 (1), p. 50; 6, t. 16, f. $23(1847-56)$. Hamps., Moths Ind. 1, p. 226 (1892).

a) Madagasische Arten.

? Cambouei Oberth., Et. Ent. 18, p. 6, t. 1, f. 17, 18 Madagaskar (1893) (Naclia). - Hamps., Cat. Lep. Phal. 1, p. 135 (1898) (huius generis?).

florida Joannis, Ann. Soc. Ent. France 75, p. 169, t. 9, Mauritius f. 1 (1906).

lucia Oberth., Et. Ent. 18, p. 6, t. 1, f. 5 (1893) (Naclia). Madagaskar - Hamps., Cat. Lep. Phal. 1, p. 135 (1898). 
quinquemacula Mab., Le Nat. 2, p. 131 (1882) (Syntomis).

Madagaskar

Perroti Oberth., Et. Ent. 18, p. 7, t. 1, f. 3, (1893) (Naclia).

sublenestrata Auriv., Ent. Tidskr. 20, p. 245 (1899).

\section{b) Paläarktisch-orientalische Arten.}

0 - aneilla L., Syst. Nat. ed. 12, p. 835 (1766) (Noctua). Esp., Schmett. 4, p. 51, t. 85, f. 1, 2 (1786). Hübn., Eur. Schmett. 3, f. 114, 245 (1802-08). - Ochs., Eur. Schmett. 3, p. 157 (1810). God., Hist. Nat. Lóp. 4, p. 379 , t. 39 , f. 2,3 (1822). - Hamps., Cat. Lep. Phal. 1, p. 134 (1898). - Spul., Schmett. Eur. 2, p. 127, t. 72, f. 17 (1906). - Borge-Rebel. Schmetterlingsb. p. 424 , t. 50, f. 2 (1910). - Seitz, Grossschmett. d. Erde, F. pal. 2, p. 42 , t. 9, i (1910). obscura Fabr., Spec. Ins. 2, p. 206 (1781) (Bombyx). quadrioculata Göze, Ent. Beitr. 4 (4) p. 126 (1783) (Tinea).

lemopicta Fourcr., Ent. Par. 2, p. 302 (1785) (Phalaena).

tripunctata Scop., Fauna \& Flora Insubr. 2, t. 21, f. 3 (1786) (Phalaena).

Biologie: Ochs., Eur. Schmett. 3, p. 158 (1810). - Frr., N. Beitr. 1, p. 60 , t. 32 , f. 2 (1833). - Wilde, Raup. Deutschl. p. 104 (1861). Rössl., Schuppenfl. Wiesb. p. 47 (1881). - Spul., Schmett. Eur. 2, p. 127, Raup. t. IX, f. 9 (1906). - Bcrge-Rolvel, Schmetterlingsb. p. 424 (1910). - Seitr, Grossschmett. d. Erde, F. pal. 2, p. 42 (1910)

- punctata Fabr., Spec. Ins. 2, p. 207 (1781) (Bombyx). - Borkh., Naturg. eur. Schmett. 3, p. 217 (1790). - Ochs., Eur. Schmett. 3, p. 159 (1810). - Frr., N. Beitr. 1, p. 61 , t. 32 , f. 3 (1833). - Mill., Icon. Descr. Chén. Lép. 1, p. 250 , t. 4, f. 11 (1861). - Hamps., Moths Ind. 1, p. 226, f. 142 (1892); Cat. Lcp. Phal. I, p. 133, f. 53 (1898). - Spul., Schmett. Eur. 2, p. 127, t. 72, f. 18 (1906). - Berge-Rebel, Schmetterlingsb. p. 424, t. 50, f. 3 (1910). - Seitz, Grossschmett. d. Erde, F. pal. 2, p. 41, t. 9, i (1910).

ancilla var. Esp., Schmett. 4, p. 51, t. 85, f. 3 (1786) (Noctua).

serva Hübn., Eur, Schmett. 3, f. 115, 226, 227 (1802-08) (Bombyx). - God., Hist. Nat. Lep. 4 , p. 381 , t. 39 , f. 4 (1822).

ab. famula Trr., N. Beitr. 2, p. 193, t. 183, f. 2, 3 (1836). - Stgr., Hor. Soc. Ent. Ross. 7, p. 106 (1871). - Hamps., Cat. Lep. Phal. I, p. 133 (1898). - Spul., Schmett. Eur. 2, p. 127 (1906). - Berge-Rebel, Schmetterlingsb. p. 244 (1910). - Seitz, Grossschmctt. d. Erde, F. pal. 2, p. 42 (1910).
Mittel- und Südcuropa (excl.Grossbritannien, Holland, ibcr. Halb. insel, Griechenland), Südural

Südeuropa

(excl.

Mittel- u.

Süd.

spanien)

nördl. bis

Wallis,

Südtirol,

Krain,

Kroatien,

Südungarn;

Siidural,

Trans-

kaspien,

Armenien,

Kleinasien,

Syrien,

Algerien

Süditalien,

Balkan-

halbinsel,

Kein-

asien etc. 
ab. hyalina Frr., N. Beitr. 5, p. 152, t. 473, f. 2 (1845). - Herr.-Schäff., Syst. Bearb. Schmett. Eur. 2, p. 51, f. 127 (1818). - Hamps., Cat. Lep. Phal. 1, p. 133 (1898). - Spul., Schmett. Eur. 2, p. 127 (1906). - Berge-Rebel, Solmetterlingsb. p. 424 (1910). - Seitz, Glossschmett. d. Erde, F. pal. 2, p. 41, t. 9, i (1910).

punctata Zell., Isis p. 16 (1847) (nec. f. typ. Fabr.). ab. juncta Hafner, Schmett. Krains p. 203, 237, t. 3, f. 33 (Carniola 1911-12).

ab. modesta Krulikowsky, Soc. Ent. 9, p. 161 (1895). - Hamps., Cat. Lep. Phal. 1, p. 134 (1898). - Spul., Schmott. Eur. 2, p. 127 (1906). - Seitz, Grossschmett. d. Erde, F. pal. 2, p. 42 (1910).

ab. ochrea Mill., Cat. Lép. Alp. Marit. p. 391 (1873). - Mém., Soc. Sc. Nat. Cannes t. 8, f. 2 (1880). - Spul., Schmett. Eur. 2, p. 127 (1906).

- Berge-Rebel, Schmetterlingsb. p. 424 (1910).

ab. ragusaria Zickert, Ent. Zcitschr. Gub. 18, p. 78 (1904); Nat. Sio. 17, p. 96 (1905). - Spul., Schmett. Eur. 2, p. 492 (1910). - Seitz, Grossschmett. d. Erdo, F. pal. 2, p. 42 (1910).

ab. servula Bcrco, Ann. Soc. Ent. France (4) 2, p. 386, t. 9 , f. 9 (1862). - Stgr., Hor. Soc. Ent. Ross. 7, p. 107 (1871). - Hamps., Cat. Lep. Phal. 1, p. 133 (1898). - Spul., Schmett. Eur. 2, p. 127 (1906). - Berge-Rebel, Schmetterlingsb. p. 424 (1910). - Seitz, Grossschmett. d. Erde, F. pal. ‘, p. 42 (1910).

v. separata Bang-Haas, Iris 19, p. 143, t. 5, f. 15 (1906). - Seitz, Grossschmett. d. Erde, F. pal. 2, p. 42 , t. 9 , i (1910).

v. parvigutta Christ., Verh. naturw. Ver. Brünn 27, p. 11 (1889). - Mém., Rom. 5, p. 13, t. 1, f. 5 (1889). - Hamps., Cat. Lep. Phal. 1, p. 134 (1898). - Spul., Schmett. Eur. 2, p. 127 (1906). - Seitz, Grossschmett. d. Erde, F. pal. 2, p. 42, t. 9 , i (1910).

v. kashmirensis Rothsch., Nov. Zool. 17, p. 441 (1910).

Biolo gi e; Mill., Icon. Descr. Chén. Lép. 1, p. 250, t. 4, f. 10 (1859-64). - Spul., Solimett. Eur. 2, p. 127, Raup. t. 10, f. 12 (1906). - BergeRebel, Schmetterlingsb. p. 424 (1910). - Seitz, Grossschmett. d. Erde, F. pal. 2, p. 42 (1910).

syntomida Stgr., Iris 4, p. 248 (1891); 6, t. 1, f. 9 (1893)

- (Naclia). - Hamps., Cat. Lep. Phal. 1, p. 134

(1898). - Seitz, Grossschmett. d. Erde, F. pal.

2, p. 42 , t. 9 , h $(1910)$.

8

\section{Tritonaclia Hamps.}

Hamps., Cat. Lep. Phal. 1, p. 135 (1898). erubescens Hamps., Ann. \& Mag. Nat. Hist. (7) 8, p. 169 (1901).

Kefersteini Butl., Cist. Ent. 3, p. 2 (1882) (Hydrusa).

Südungarn,

Balkan-

halbinsel,

Klein-

asien,

Syrien eto.

Krain

Südrussland

Südfrank-

reich etc.

Süditalien

Südfrank-

reich,

Catalonien,

Portugal,

Algerion

etc.

Algerien

Transkaspien

Kashmir

Mesopota.

mien

Brit.

Ostafrika

Madagaskar 


\section{IIfegauaclia Auriv.}

Auriv., Ent. Tidskr. 13, p. 290 (1892). - Hamps., Cat. Lep. Phal. 1, p. 136 (1898).

d t carnea Hamps., Cat. Lep. Phal. 1, p. 136, t. 6, f. 27 (1898).

-s sippia Plötz, Stett. Ent. Zeitg. 41, p. 78 (1880) (Naclia).

- Mab., Ann. Soc. Ent. France 10 (6), p. 37

(1890). - Hamps., Cat. Lep. Phal. 1, p. 136, f. 55 (1898). - Auriv., Ark. Zool. (4) 2, p. 28 (1904).

Biologie: Auriv., Ark. Zool. (4) 2 p. 28 (1904).

2

Gabun, Congo (M.

(C. Vind.) Angola,

Sierra Leone

(M. C.

Vind.),

Nigeria,

Kamerun,

Uganda

\section{Thyretes Boisd.}

Boisd., Voy. Delegorgue 2, p. 596 (1847). - Butl., Journ. Linn. Soc. Lond., Zool. 12, p. 359, t. 28, f. 11 (1876). - Hamps., Cat. Lep. Phal. 1, p. 137 (1898).

- caffra Wallengr., Wien. Ent. Monatsschr. 7, p. 138 (1863); Svenska Akad. Handl. 5 (4), p. 11 (1865). Hamps., Cat. Lep. Phal. 1, p. 138, f. 57 (1898). Biologie: Barrett, Ent. Monthl. Mag. (2) 12, p. 193 (1901).

hippotes Cram., Pap. Exot. 3, p. 166, t. 286, f. A (1780) (Sphinx). - Hamps., Cat. Lep. Phal. 1, p. 137, f. 56 (1898).

misn Strand, Ann. Soc. Ent. Belg. 55, p. 146 (1911).

montana Boisd., Voy. Delegorgue 2, p. 597 (1847). Wallengr., Svenska Akad. Handl. 5 (4), p. 10 (1865). - Hamps., Cat. Lep. Phal. 1, p. 138, t. 6 , f. 7 (1898).

Ionteiroi Butl., Journ. Linn. Soc. Lond., Zool. 12, p. 359 (1876). - Hamps., Cat. Lep. Phal. 1, p. 139, t. 5 , f. 9 (1898).

negus Oberth., Et. Ent. 3, p. 31, t. 3, f. 2 (1878). Hamps., Cat. Lep. Phal. 1, p. 139 (1898).

ab. phasma Butl., Proc. Zool. Soc. Lond. p. 846 (1896). - Hamps., Cat. Lep. Phal. 1, p. 139 (1898).

trichaetiformis Zerny, Iris 26, (1912) (ined.).

Kapland, Transvaal, Delagoabai, Deutsch Südwest afrika

Kapland

Togo

Kapland, Zululand

Angola, Congo

Abyssinien

Portug.-

Senegam-

bien,

Sicrra

Leone,

Nyassaland,

Deutsch-

Südwest-

afrika (M.

C. Vind.)

Zanzibar

7

Thyrogonia Hamps.

Hamps., Cat. Lep. Phal. 1, p. 139 (1898).

efulensis Holl., Ent. News 9, p. 12 (1898) (Syntomis). Kamerun 


\section{Eressades Beth.-Baker.}

Beth.-Baker, Ann. \& Mag. Nat. Hist. (8) 7, p. 531 (1911). flavipunctata Beth.-Baker, Ann. \& Mag. Nat. Hist. (8) Angola 1 7, p. 531 (1911).

\section{Apisa Walk.}

Walk., List. Lep. Ins. Br. Mus. 4, p. 916 (1855). - Hamps., Cat. Lep. Phal.

1, p. 140 (1898).

A nace Walk., List. Lep. Ins. Br. Mus. 7, p. 1719 (1856), - Auriv., Ent. Tidskr. 19, p. 186 (1898).

a urantiaca Rothsch., Nov. Zool. 17, p. 441 (1910)*).

- canescens Walk., List. Lep. Ins. Br. Mus. 4, p. 917 (1855). - Hamps., Cat. Lep. Phal. 1, p. 142, f. 59 (1898).

pallota Plötz, Stett. Ent. Zeitg. 41, p. 78 (1880) (Psychotoe).

cinereocostata Holl., Psyche 6, p. 394 (1893). cana Holl., Psyche 6, p. 394, t. 10, f. 33 (1893). Biologie: Barrett. Ent. Monthl. Mag. 13 (2), p. 123 (1902).

chrysopyga Plötz, Stett. Eint. Zeitg. 41, p. 79 (1880) (Syntomis). - Hamps., Cat. Lep. Phal. 1, p. 141, t. 5, f. 11 (1898). - Auriv., Ark. Zool. $2(4)$, p. 28 (1904).

? cleta Plötz, Stett. Ent. Zeitg. 41, p. 79 (1880) (Syntomis). - Hamps., Cat. Lep. Phal. 1, p. 142 (1898) (huius generis?).

connexa Walk., List Lep. Ins. Br. Mus. 1, p. 246 (1854) (Euchromia). - Butl., Illustr. Het. Br. Mus. 1 , p. 18, t. 8, f. 1 (1877). - Hamps., Cat. Lep. Phal. 1, p. 140 (1898).

crenophylax Holl., Psyche 6, p. 393 (1893) (Symtomis). - Hamps., Cat. Lep. Phal. 1, p. 143, t. 5, f. 18 (1898).

Kerri Holl., Ent. News 9, p. 12 (1898) (Syntomis). Hamps., Cat. Lep. Phal. 1, p. 141, t. 5, f. 12 (1898).

leucogastra Holl., Psyche 6, p. 393 (1893) (Symtomis). - Hamps., Cat. Lep. Phal. 1, p. 141, t. 5, f. 10 (1898).

melaleuca Holl., Ent. News 9, p. 13 (1898) (Anace). Hamps., Cat. Lep. Phal. 1, p. 143, t. 5, f. 16 (1898).

metaretioides Hamps., Ann. \& Mag. Nat. Hist. (7) 19, p. 224 (1907); Trans. Zool. Soc. Lond. 19, t. 4,

Kamerun, Congo

Natal, Transvaal, Deutsch-

Südwestu.Ostafrika, Brit.-

Ostafrika, Franz.Congo,

Sierra

Leone

Kamerun

,

Fernando Po

Franz.-

\section{Congo}

Kamerun

Franz. -

\section{Congo}

Kamerun

Uganda f. 3 (1909).

*) abdominalis Ehrmann. Can. Ent. 26, p. 70 (1894) (Syntomis). - Hamps., Cat. Lep. Phal, 1, p. 141 (1898) (Apisa) seo. Dyar, Can. Ent. 31, p. 8s (1899) ad Zyga6nidas referenda est. 
- monotica Holl., Psyche 6, p. 395, t. 10, f. 31 (1893) (Anace). - Hamps., Cat. Lep. Phal. 1, p. 142, t. 5, f. 15 (1898).

parachoria Holl., Psyche 6, p. 395, t. 10, f. 32 (1893) (Anace). - Hamps., Cat. Lep. Phal. 1, p. 147, t. 5, f. 28 (1898). - Auriv., Ark. Zool. (4) 2, p. 29 (1904).

perpusilla Walk., List Lep. Ins. Br. Mus. 7, p. 1720 (1856) (Anace). - Hamps., Cat. Lep. Phal. 1, p. 144 , t. 5 , f. 19 (1898).

quadrinaculata Zcrny, Iris 26 (1912) (ined.).

Rendalli Rothseh., Nov. Zool. 17, p. 441 (1910).

Sjoestedti Auriv., Ark. Zool. (4) 2, p. 28, f. 24 (1904). subcanescens Rothsch. Nov. Zool. 17, p. 442 (1910). tristigma Miab., Ann. Soc. Ent. Belg. 37, p. 58 (1893) (Naclia); Nov. Lep. p. 148, t. 21, f. 2 (1895). 18 - Hamps., Cat. Lep. Phal. 1, p. 142 (1898).

Altcalabar, Kamerun, Franz.Congo

Kamerun, Franz.Congo, Congo (M. C. Vind.)

? Sierra Leone

Congo Brit.-Zentral. afrika

Kamerun Patria? Sierra Leone

\section{Metaretia Walk.}

Walk., List Lep. Ins. Br. Mus. 3, p. 769 (1855). - Hamps., Cat. Lop. Phal. 1, p. 143 (1898).

Zagaris Walk., List Lep. Ins. Br. Mus. 5, p. 1096 (1855). Decimia Walk., List Lep: Ins. Br. Mus. 7, p. 1717 (1856). Hebena Walk., List Lep. Ins. Br. Mus. 7, p. 1722 (1856). Hexaneura Wallengr., Wien. Ent. Monatsschr. 4, p. 164 (1860). - Srenska, Akad. Handl. 5 (4), p. 50 (1865).

benitensis Holl., Psyche 6, p. 395 (1893). - Hamps., Cat. Lep. Phal. 1, p. 148, t. 5, f. 27 (1898).

burraiSchaus. \& Clements, Lep. Sierra Leone p. 23, t. 1, f. 6 (1893) (Anace). - Hamps., Cat. Lep. Phal, 1, p. 147 (1898).

contrasta Beth.-Baker, Ann. \& Mag. Nat. Hist. (8) 7, p. 533 (1911).

crassa Fold., Reise Nor., Lep. 2, p. 10, t. 99, f. 16 (1868) (Zagaris). - Hamps., Cat. Lep. Phal. 1,.p. 149 (1898).

meteus Walk., List Lep. Ins. Br. Mus. 5, p. 1096 (1855) (nec Cram.).

diversa Beth.-Baker, Ann. \& Mag. Nat. Hist. (8) 7, Angola p. 532 (1911).

Erlangeri Rothsch., Nov. Zool. 17, p. 442 (1910).

lerrigera Druce, Ann. \& Mag. Nat. Hist. (8) 5, p. 395 (1910).

flaviceps Hamps., Cat. Lep. Phal. 1, p. 147, t. 5, f. 21 (1898).

flaviciliata Hamps., Ann. \& Mag., Nat. Hist. (7) 19, p. 225 (1907); - Trans. Zool. Soc. Lond. 19, t. 4, f. 40 (1909).

flavicincta Auriv., Öfvers. Svenska Akad. Förh. p. 1057 (1900).

Kamerun

Sierra Leone, Congo, Uganda

Angola

Kapland

Abyssinien, Angola

Kamerun "

Uganda

Congo 
flavivena Hamps., Aun. S. Afric. Mus. 2, p. 40 (1900); Ann. \& Mag. Nat. Hist. (7) 8, p. 169 (1901).

fulvia Hamps., Ann. \& Mag. Nat. Hist. (7) 8, p. 170 (1901).

fusea Hamps., Ann. \& Mag. Nat. Hist. (7) 8, p. 169 (1901).

Inematica Holl., Psyche 6, p. 396 (1893). - Hamps., Cat. Lep. Phal. 1, p. 147, t. 5, f. 26 (1898).

haematoëssa Holl., Psychc 6, p. 396 (1893). - Hamps., Cat. Lep. Phal. 1, p. 146, t. 5, f. 17 (1898). Oberth., Ann. Soc. Ent. France 79, p. 469 , t. 17, f. 7 (1911).

liaematosphages Holl., Psyche 6, p. 396 (1893). - Hamps., Cat. Lep. Phal. 1, p. 146, t. 5, f. 25 (1898).

haemotricha Hamps., Ann. \& Mag. Nat. Hist. (7) 15, p. 426 (1905).

ineensa Walk., List Lep. Ins. Br. Mus. 31, p. 316 (1864) (Anace). - Hamps., Cat. Lep. Phal. 1, p. 149, t. 6 , f. 13 (1898).

inconspicua Holl., The Ent. 25, Suppl. p. 93 (1892); Proc. U. S. Nat. Mus. 18, p. 250, t. 8, f. 3 (1896). - Hamps., Cat. Lep. Phal. 1, p. 148, t. 6, f. 11 (1898).

invaria Walk., List Lep. Ins. Br. Mus. 7, p. 1720 (1856) (Anace). - Hamps., Cat. Lep. Phal. 1, p. 146, t. 5, f. 20 (1898). - Rothsch., Nov. Zool. 19, p. 122 (1912) (gen. Balacra).

syntomia Plötz, Stett. Ent. Zeitg. 41, p. 85 (1880) (Plegapteryx). - Möschl., Abb. Senckenb. naturf. Ges. 15 , p. 72 (1887).

silacea Plötz, Stett. Ent. Zeitg. 41,, 4 p. 86 (1880) (Plegapteryx).

melinos Mab., Ann. Soc. Ent. France (6) 10 , p. 37 (1890) (Thyretes).

ab. erubescens Walk., List Lep. Ins. Br. Mus. 31, s. p. 315 (1864). - Hamps., Cat. Lep. Phal. 1, p. 146 (1898).

pareclecta Holl., Psjebe 6, p. 395 (1893).

ab. lutea Holl., Psyche 6, p. 396 (1893). - Hamps., Cat. Lep. Phal. 1, p. 146 (1898).

v. Conradti Oberth., Ann. Soc. Ent. France 79, p. 468, t. 17, f. 9 (1911).

- Iateritia Herr.-Schäff., Ausscrcurop. Schmett. p. 72, f. 274 (1855) (Automolis). - Hamps., Cat. Lep. Phal. 1, p. 145, f. 60 (1898).

rubra Walk., List Lep. Ins. Br. Mus. 7, p. 1720 (1856) (Anace).

ve nosa Walk., List Lep. Ins. Br. Mus. 7, p. 1723 (1856) (Hebena).
Mashonaland, Portug. u.

Brit.-

Ostafrika

Brit.- $\mathbf{u}$.

Deutsch-

Ostafrika

Brit.-

Ostafrika

Nigeria,

Franz.-

Congo,

Brit.-

Ostafrika

Franz.-

Congo

Abyssinien

Patria?

Kamerun,

Franz.-

Congo,

Congo,

Angola,

Deutsch-

Ostafrika

Sierra Leone

bis Congo

Kamerun

Kapland bis Gallaland u. Congo (M. C. Vind.) 
cinnamomea Wallengr., Wien. Ent. Monatsschr. 4, p. 164 (1860) (Hexaneura); Svenska Akad. Handl. 5 (4), p. 51 (1865).

unicolor Oberth., Ann. Mus. Genova 15, p. 186 (1880) (Automolis).

ab. Kelleni Snell., Notes Leyd. Mus. 8, p. 1 (1886) (Automolis); Tijdschr. v. Ent. 29, p. 224, t. 8, f. 1 (1886). - Hamps., Cat. Lep. Phal. 1, p. 145 (1898).

meteus Cram., Pap. Exot. 4, p. 109, t. 347, f. B. (1782) $(\operatorname{Sphinx})$. - Hamps., Cat. Lep. Phal. 1, p. 148 (1898).

bicolora Walk., List. Lep. Ins. Br. Mus. 7, p. 1718 (1856) (Decimia).

Biologie: Fawcett, Trans. Zool. Soc. Lond. 17, p. 178 , t. 7 , f. $15-19(1903)$.

nigriceps Auriv., Ark. Zool. (4) 2, p. 30 (1904).

noctis Druce, Ann. \& Mag. Nat. Hist. (8) 5, p. 394 (1910).

pallens Beth.-Baker, Ann. \& Mag. Nat. Hist. (8) 7, p. 532 (1911).

pallida Hamps., Ann. \& Mag. Nat. Hist. (7) 8, p. 170 (1901).

phaeoptcra Hamps., Ann. \& Mag. Nat. Hist. (8) 4, p. 344 (1909).

pulverea Hamps., Ann. \& Mag. Nat. Hist. (7) 19, p. 225 (1907); Trans. Zool. Soc. Lond. 19, t. 4, f. 4 (1909).

pumila Hamps., Ann. \& Mag. Nat. Hist. (8) 4, p. 344 (1909).

rosncen Beth.-Baker, Ann. \& Mag. Nat. Hist. (8) 7, p. 532 (1911).

rubribasa Beth.-Baker, Ann. \& Mag. Nat. Hist. (8) 7, p. 533 (1911).

rubrilineata Beth.-Baker, Ann. \& Mag. Nat. Hist. (8) 7, p. 533 (1911).

rubripuncta Hamps., Cat. Lep. Phal. 1, p. 147, t. 5, f. 4 (1898).

v. rosea Auriv., Ark. Zool. (1) 3, p. 13 (1905); rubripuncta ab. 1 Hamps., Cat. Lep. Phal. 1, p. 148 (1898).

rufescens Walk., List. Lep. Ins. Br. Mus. 3, p. 769 (1885). - Hamps., Cat. Lep. Phal. 1, p. 148, t. 5 f. 6 (1898).

maculifera Wallengr., Wien. Ent. Monatsschr. 4, p. 164 (1860) (Hexaneura). - Svenska, Akad. Handl. 5 (4), p. 51 (1865).

ab. paremphares Holl., Psyche 6, p. 395 (1893). - Hamps., Cat. Lep. Phal. 1, p. 148 (1898).

Biologie: Barrett, Ent. Monthl. Mag. (2) 12, p. 285 (1901); 14 (2), p. 140 (1903).

sarcosoma Hamps, Ann. \& Mag. Nat. Hist. (7) 8, p. 170 (1901).

Taymansi Rothsch., Nov. Zool. 17, p. 442, (1910).

Kapland, Transvaal, Natal,

Somaliland (Pagenst.)

Kamerun Abyssinien

Angola

Brit.-

Ostafrika Congo

Uganda

Angola

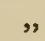

Gabun

Congo

Kapland, Natal, Transvaal, Mozambique, DeutschOstafrika, Franz.Congo

Brit.Ostafrika uniformis Beth.-Baker, Ann. \& Mag. Nat. Hist. (8) 7, p. 534 (1911).

Congo

Angola 


\section{Balacra Walk,}

Walk., List Lep. Ins. Br. Mus. 7, p. 1721 (1856). - Auriv., Ent. Tidskr. 19, p. 186 (1898). - Rothsch., Nov. Zool. 19, p. 119 (1912).

Pseuda piconoma Auriv., Ent. Tidskr. 2, p. 46 (1881). - Hamps., Cat. Lep. Phal. 1, p. 149 (1898).

Megapisa Auriv., Ark. Zool. (4) 2, p. 29 (1901).

affinis Rothsch., Nor. Zool. 17, p. 442 (1910) (Pseuda- Congo piconoma); 19 , p. 122, t. 1, f. 20 (1912).

Batesi Druce, Ann. \& Mag. Nat. Hist. (8) 5, p. 393 (1910) (Pseudapiconoma). - Rothsch., Nov. Zool. 19, p. 120 , t. 2, f. 25, 26 (1912).

จ. congoensis Rothsch., Nor. Zool. 17, p. 443 (1910) (Pseudapiconoma). - Rothsch., Nov. Zool. 19, p. 120, t. 2, f. 27 (1912).

v. Ugandae Rothsch., Nov. Zool. 17, p. 443 (1910) (Pseudapiconoma); 19, p. 120, t. 2, f. 34 (1912).

caeruleifascia Walk., List Lep. Ins. Br. Mus. 7, p. 1721 (1856). - Hamps., Cat. Lep. Phal. 1, p. 145, t. 5, f. 13 (1898). - Rothsch., Nov. Zool. 17, p. 443 (1910); 19, p. 119, t. l, f. 8, 9 (1912).

Ehrmanni Hamps., Cat. Lep. Plıal. 1, p. 145 (1898) (ncc Holl.).

compsa Jord., Nov. Zool. 11, p. 441 (1904) (Pseuda. piconoma). - Rothsch., ib. 19, p. 121, t. 2 , f. 42 (1912).

Conralti Oberth., Ann. Soc. Ent. France 79, p. 471, t. 17, f. 12 (1911) (Pseudapiconoma).

duphueua Hamps., Cat. Lep. Phal. 1, p. 150, f. 62 (1898) (Pseudapiconoma). - Rothsch., Nov, Zool. 19, p. 122, t. 1, f. 19 (1912).

Ehrmanni Holl., Psyche 6, p. 535 (1893) (Automolis). - Rothsch., Nov. Zool. 17, p. 443 (1910); 19, p. 119 , t. 1, f. 10,11 (1912).

elegans Auriv., Ent. Tidskr. 13, p. 200 (1892). - Hamps., Cat. Lep. Phal. 1, p. 152, f. 63 (1898). - Auriv., Ark. Zool. (1) 3, p. 13 (1905). - Hamps., Ann. \& Mag. Nat. Hist. 19 (7), p. 225 (1907). - Rothsch., Nov. Zool. 19, p. 121, t. 1, f. 4, 5 (1912).

damalis Holl., Psyche 6, p. 397, t. 10, f. 29 (1893). v. Curriei Dyar, Journ. New York Ent. Soc. 7, p. 174 (1899) (Pseudapiconoma).

fenestrata Jord., Nov. Zool. 11, p. 442 (1904) (Pseuda. piconoma). - Rothsch., ib. 19, p. 121, t. 2, f. 35 (1912).

- flavimacula Walk., List Lep. Ins. Br. Mus. 7, p. 1722 (1856). - Hamps., Cat. Lep. Phal. 1, p. 150, t. 6, f. 12 (1898). - Auriv., Ark. Zool. (4) 2, p. 32, f. 27 b. (1904). - Rothsch., Nov. Zool. 19, p. 120 , t. 2 , f. 28,29 (1912).

v. decora Oberth., Ann. Soc. Ent. France 79, p. 470 , t. 17, f. 10, (1911) (Pseudapiconoma).

furva Hamps., Ann. \& Mag. Nat. Hist. (8) 8, p. 394 (1911). - Rothsch., Nov. Zool. 19, p. 121, t. 1, f. 3 (1912).

Kamerun

Congo

Uganda

Sierra Leone, Kamerun, Congo

Angola

Kamerun

Nigeria, Kamerun, Franz.Congo

Liberia

Kamerun, Congo, Uganda

Liberia

Angola

Ashanti, Nigeria, Kamerun Franz.Congo

Kamerun

Goldküste, Ashanti 
rubricincta ab. 1 Hamps., Cat. Lep. Phal. 1, p. 151 (1898) (Pseudapiconoma).

germana Rothsch., Nov. Zool. 19, p. 119, t. 2, f. 24 (1912). gloriosa Jord., Nov. Zool. 11, p. 441 (1904) (Pseuda piconoma). - Rothsch., ib. 19 , p. 121, t. 2, f. 31 (1912).

Guillemei Oberth., Ann. Soc. Ent. France 79, p. 469 , t. 17, f. 11 (1911) (Pseudapiconoma).

haemalea Holl., Psyche 6, p. 397 (1893). - Hamps., Cat. Lep. Phal. 1, p. 151 (1898). - Rothsch., Nov. Zool. 19, p. 120, t. 1, f. 17, 18 (1912).

herona Druce, Proc. Lool. Soc. Lond. p. 669 (I887) (Anace). - Hamps., Cat. Lep. Phal. 1, p. 149, f. 61 (1898). — Rothsch., Nov. Zool. 19, p. 121, t. $1, \mathrm{f} .6,7$ (1912).

Humplureyi Rothsch., Nov. Zool. 19, p. 119 , t. 1, f. 14 (1912).

intermedia Rothsch., Nov. Zool. 19, p. 120, t. 2, f. 23 (1912).

melaenn Hamps., Ann. \& Mag. Nat. Hist. (7) 15, p. 426 (1905) (Pseudapiconoma).

nigripennis Auriv., Ark. Zool. (4) 2, p. 30 (1904) (Hega. pisa).

ochracen Walk., Proc. Nat. Hist. Soc. Glasgow 1, p. 331 (1869). - Hamps., Cat. Lep. Phal. 1, p. 151 (1898). - Rothsch., Nov. Zool. 19, p. 120, t. 1, f. $15,16(1912)$.

Preussi Auriv., Ark. Zool. (4) 2, p. 31, f. 26 (1904) (Pseudapiconoma). - Rothsch., Nov. Zool. 17, p. 444 (1910); 19, p. 120 (1912).

speculigera Grünberg, Deutsch. Ent. Zeitschr. p. 434 , t. 4 , f. 5 (1907) (Pseudapiconoma).

ab. brunnea Grünberg, Deutsch. Ent. Zeitschr. p. 434, t. 4, f. 4 (1907) (Pseudapiconoma). Rothsch., Nov. Zool. 19, p. 121, t. 2, f. 40 (1912). la ureola Druce, Ann. \& Mag. Nat. Hist. (8) 5, p. 393 (1910) (Pseudapiconoma).

ab. oblitterata Grünberg, Deutsch. Ent. Zeitschr. p. 435 (1907) (Pseudapiconoma).

pulchra Auriv., Ent. Tijdskr. 13, p. 200 (1892) (Pseudapiconoma). - Rothsch., Nov. Zool. 19, p. 121, t. 2, f. 32, 33 (1912).

glagoessa Holl., Psyche 6, p. 396, t. 10, f. 28 (1893). - Hamps., Cat. Iep. Phal. 1, p. 151, t. 6 , f. 14 (1898).

Rattrayi Rothsch., Nov. Zool. 17, p. 144 (1910) (Metapiconoma); 19 , p. 120 , t. 1, f. 12,13 (1912).

rubricincta Holl., Psyche 6, p. 396, t. 10, f. 27 (1893). - Hamps., Cat. Lep. Phal. 1, p. 151, t. 6, f. 15 (1898). - Rothsch., Nov. Zool. 19, p. 121, t. 1, f. 1,2 (1912).

rubrostriata Auriv., Ent. Tidskr. 19, p. 185 (1898). Rothsch., Nov. Zool. 19, p. 122, t. 2, f. 37 (1912).

rubrovitta Auriv., Ark. Zool. (4) 2, p. 31, f. 25 (1904) (Metarctia). - Rothsch., Nov. Zool. 19, p. 121, t. 2, f. 41 (1912).

stigmatiea Grünberg, Dcutsch. Ent. Zeitschr. p. 435, Uganda t. 4, f. 6 (1907) (Pseudapiconoma).

Sierra Leone

Angola

Tanganyika-

see

Franz.-Congo

Kamerun

Nigeria, Congo

Sierra Ieone

Uganda

Zentralafrika, Congo

Congo

Kamerun

\section{Kamerun, \\ Franz. - \\ Congo, \\ Congo (M. \\ C. Vind.)}

Uganda

Franz.

Congo

Togo

Kamerun 
testacea Auriv., Ent. Tidskr. 2, p. 46, f. 1 (1881) (Pseudapiconoma); Ark. Zool. (4) 2, p. 32, f. 27a (1904). - Rothsch., Nov. Zool. 19, p. 120, t. 1, f. 21, 22 (1912).

umbra Druce, Ann. \& Mag. Nat. Hist. (8) 5, p. 394 (1910) (Pseudapiconoma). - Rothsch., Nov. Zool. 19, p. 120 , t. 2 , f. 30 (1898).

vitreatu Rothsch., Nov. Zool. 17, p. 445 (1910) (Pseudapiconoma); 19, p. 121, t. 2, f. 36 (1912).

vitrina Oberth., Et. Lép. conıp. 3, p. 95, t. 11, f. 5 (1909) 32 (Pseudapiconoma).

Paxamelisa Auriv. Auriv., Ark. Zool. (1) 3, p. 13 (1905). lophura Auriv., Ark. Zool. (1) 3, p. 13, t. 1, f. 2 (1905). lophuroides Oberth., Ann. Soc. Ent. France 79, p. 468, 2 t. 17 , f. 13 (1911).

Kamerun, Gabun, Fernando Po

Kamerun<smiles></smiles>

Patria?

Kamerun

\section{Pseudomelisa Hamps.}

(Pseudmelisa) Hamps., Proc. Zool. Soc. Lond. p. 391 (1910). chalybea (chalybsa) Hamps., Proc. Zool. Soc. Lond. Congo 1 p. 391 , t. 36 , f. $5(1910)$.

\section{Melisa Walk.}

Walk., List Lep. Ins. Br. Mus. 1, p. 264 (1854). — Hamps., Cat. Lep. Phal. 1, p. 152 (1898).

atavistis Hamps., Ann. \& Mag. Nat. Hist. (8) 8, p. 395 Goldküste (1911).

diptera Walk., List Lep. Ins. Br. Mus. 1, p. 265 (1854) (Euchromia). - Butl., Illustr. Het. Br. Mus. 1, p. 19 , t. 9, f. 10 (1877). - Hamps., Cat. Lep. Phal. 1, p. 152, f. 64 (1898). croceipes Auriv., Ent. Tidskr. 13, p. 200 (1892) 2 (Balacra)*).

\section{Phacosphecia Hamps.}

Hamps., Cat. Lep. Phal. 1, p. 153 (1898).

opaca Walk., List. Isep. Ins. Br. Mus. 7, p. 1620 (1856) (Myrmecopsis). - Hamps., Cat. Lop. Phal. 1, p. 153 , f. 65 (1898).

ves pa Herr.-Schäff., Aussscreur. Schmett. p. 73, f. 426 (1856) (Pseudosphex).

Congo

Kamerun

Biologio: Gerstäcker, Stett. Ent. Zeitg. 24, p. 431

(1863) (Mimicry). - Haasc, Bibl. Zool. S (2), 1

p. 77 (1893) (Mimiary).

*) "Melisa" grandis Holl., Psycho 6, p. 394 (1893) sec. Jord., The Ent. 40, p. 127 (1907), ad Zygaenidas (Gen. Melisomimas Jord.) pertinet.

Ashanti,? Altcalabar, Gabun, Congo
Pará, Santa Catharina (M. C. Vind.) 
Orcynia Walk.

Walk., List Lep. Ins. Br. Mus. 1, p. 274 (1854). — Hamps., Cat. Lep. Phal. 1, p. 154 (1898).

ealearata Walk., List Lep. Ins. Br. Mus. 1, p. 274 (1854) (Euchromia). - Butl., Illustr. Het. Br. Mus. 1, p. 26, t. 9, f. 11 (1877). - Hamps., Cat. Lep. 1 Phal. 1, p. 154, f. 66 (1898).

Amazonas

(M. C.

Vind.),

Pará

\section{Psendosphex Hübn.}

Hülon. Verz. bek. Sclumett. p. 127 (1826). Zutr. Exot. Schmett. 1, p. 13 (1827). - Burm., Deser. Phys. Rep. Argent. 5, p. 372 (1878). - Hamps., Cat. Lep. Phal. 1, p. 154 (1898).

Myrmecopsis Newm., Zool. 8, Append. p. CXXII (1850). - Butl., Journ. Linn. Soc. Lond., Zool. 12, p. 380, t. 27, f. 8, 9 (1876). Möschl., Verh. zool.-bot. Ges. Wien 27, p. 634 (1877).

Sphecopsis Feld., Reise Nov. Lep. Atlas p. 9 (1869).

Biologie: Haase, Bibl. Zool. (2) 8, p. 77 (1893) (Mimicry).

aurlfer(-a) Klages, Proc. U. S. Nat. Mus. 29, p. 531 (1906).

cuturensis Klages, Proc. U. S. Nat. Mus. 29, p. 532 (1906). deceptans Zerny, Iris 26 (1912) (ined.).

exul (exsul) Rothsch., Nov. Zool. 18, p. 24 (1911).

Garleppi Rothsch., Nov. Zool. 18, p. 24 (1911).

hyalozonus(-a) Feld., Reise Nov., Lep. 2, p. 9, t. 102, f. 29 (1869) (Sphecopsis). - Hamps., Cat. Lep. Phal. 1, p. 156 (1898).

lehneumoneus(-a) Herr.-Schäff., Aussereur. Schmett. p. 73 , f. 225 (1854). - Hamps., Cat. Lep. Phal. 1, p. 156 (1898).

polybioides Burm., Descr. Phys. Rep. Argent. 5 , p. 372 (1878).

ab. crabronis Druce, Biol. Centr. Am., Het. 1, p. 54, t. 8, f. 9 (1881) (Myrmecopsis). - Hamps. Cat. Lep. Phal. 1, p. 156 (1898).

Biologie: Haase, Bibl. Zool. (2) 8 , p. 77 , t. 13 , f. 98 (1893) (Mimiery). - Schrottky, Iris 22, p. $127-129$ (1909) (Mimiery).

Jonesl Kaye, The Ent. 44, p. 142 (1911).

Klagesi Rothsch., Nov. Zool. 18, p. 24 (1911).

Iaticinctus(-a) Hamps., Cat. Lep. Phal. 1, p. 155, t. 6, f. 8 (1898).

fasciolatum Druce, Biol. Centr. Am., Het. 1, p. 54 (1884) (p. p.) (Sphecosoma) (nec Butl.).

noverea Schaus, Journ. New York Ent. Soc. 9, p. 40 (1901).

Biologie: Sehrottky, Iris 22, p. 127-128 (1909) (Mimicry).

pollstes Hübn., Zutr., Exot. Schmett. 1, p. 12, f. 39, 40 (1827). - Druce, Biol. Centr. Am. Het. 1, p. 53 (1884); 2 p. 341 (1897). - Hamps., Cat. Lep. Phal. 1, p. 155, f. 68 (1898).

Venezuela, Colombia

(M.C.Vind.)

Venezucla

Santa Catha. rina

? Südbrasilien

Bolivien

Colombia

Guatemala, Panama, Bolivia (M.' C. Vind.),

Süd-

brasilien,

Paraguay,

Uruguay,

Argentinien

São Paulo

Venezuela

Guatemala

Paraná, Paraguay

Mexico,

Guatemala, Venezuela, Surinam, 
eumenides Newm., Zool. 8, Append p. CXXII (1850) (Myrmecopsis).

v. melanogen Dyar, Proc. U. S. Nat. Mus. 38 , p. $229(1910)$.

Biologie: Gerstäcker, Stett. Ent. Zeitg. 24, p. 431 (1863) (Mimiery). - Druce, Biol. Centr.-Am. Het. 1, p. 53 (1884). - Hasse, Bibl. Zool. (2) 8, p. 77 (1893) (Mimicry).

polybin Kaye, The Ent. 44, p. 142 (1911).

rubripalpus Hamps., Ain. ․ Mag. Nat. Hist. (7) 8, p. 170 (1901).

serieeus(-a) Schrottky, Iris 24, p. 148 (1910).

Steinbachi Rothsch., Nov. Zool. 18, p. 24 (1911).

strigosus(-a) Druce, Biol. Centr.-Am., Het. 1, p. 46, t. 8, f. 3 (1884); 2, p. 335 (1896) (Amycles). - Hamps., Cat. Lep. Phal. 1, p. 155, f. 67 (1898).

Biologie: Druce, Biol. Centr.-Am., Het. 1, p. 46 17 (1884).

\section{Sphecosoma Butl.}

Butl., Journ. Liun. Soc. Lond., Zool. 12, p. 381 (1876). - Möschl., Verh. zool.-bot. Ges. Wien 27, p. 634 (1877). - Hamps., Cat. Lep. Phal. 1, p. 157 (1898).

Biologie: Haase, Bibl. Zool. (2) 8, p. 77 (1893) (Mimiery).

abdominale(-is) Schaus, Proc. U. S. Nat. Mus. 29, p. 184 (1905).

abnorne(-is) Hamps., Cat. 1.cp. Phal. 1, p. 159, f. 71 (1898).

angustatum(-a) Möschl., Verh. zool.-bot. Ges. Wien 27 p. 634, t. 8, f. 2 (1877). - Hamps., Cat. Lep. Phal. 1, p. 157, f. 69 (1898).

testaceum Druce, Biol. Centr.-Am., Het. 1, p. 54, t. 6, f. 27 (1884) (nec Walk.).

gracile(-is) Klages, Proc. U. S. Nat. Mus. 29, p. 532 (1906).

v. nigriferum Dyar, Proc. U. S. Nat. Mus. 38, Mexico p. 229 (1910).

Biologie: Haase, Bibl. Zoul. (2) 8, p. 77, t. 13, f. 94 (1893) (Mimicry).

arctatum Walli., List Lep. Ins. Br. Mus. 31, p. 95 (1864) (Pseudosphex). - Hamps., Cat. Lep. Phal. 1, p. 158 , f. 70 (1898).

auruntiipes Rothsch., Nov. Zool. 18, p. 25 (1911).

poguatum(-a) Walk., List Lep. Ins. Br. Mus. 7, p. 1619 (1856) (Pseudosphex). - Hamps., Cat. Lep. Phal. 1, p. 159 , f. 72 (1898).

fasciolatum Butl., Journ. Linn. Suc. Lond., Zool. 12, p. 381 (1876). - Druce, Biol. Centr.-Am., Het. 1, p. 54 (1884) (context. pr. p.); 2, p. 341

Paraná

São Paulo

Paraguay

Venezuela, Bolivia

Mexico, Guatemala
Venezuela

Unt. Amazonas Guatemala, Panama, Surinam, Venezuelà

\section{Amazonas}

Venezuela, Bolivia, Paraguay

Mexico, Colombia, Amazonas 
deceptrix Hamps., Cat. Lep. Phal. 1, p. 158 (1898).

fasciolatum Druce, Biol. Centr. - Am., Het. 1, p. 54 (pr. p.), t. 9, f. 26 (1884) (nec Butl.)

Bíologie: Haase, Bibl. Zool. (2) 8, p. 77, t. 13, f. 26 (1893) (Mimicry).

ecuadorum(-a) Druce, Proc. Zool. Soc. Lond. p. 376 (1883). - Hamps., Cat. Lep. Phal. 1, p. 160, t. 6 , f. 24 (1898).

Mathani Rothsch., Nov. Zool. 18, p. 25 (1911).

melanotum Hamps., Cat. Lep. Phal. 1, p. 161, t. 7, f. 4 (1898).

melanoperum (melapera) Dogn., Ann. Soc. Ent. Belg. 53 , p. 213 (1909).

melissa Schaus, Journ. New York Ent. Soc. 4, p. 121 (1896). - Hamps., Cat. Lep. Phal. 1, p. 160, t. 6 , f. 4 (1898).

meridionale Schrottky, Iris 24, p. 148 (1910).

Biologie: Schrottky, Iris 24, p. 148 (1910).

metamela Hamps., Ann. \& Miag. Nat. Hist. (7) 15, p. 426 (1905)

nigriceps Hamps., Ann. \& Mag. Nat. Hist. (7) 11, p. 340 (1903).

nigricorne(-is) Rebel, Bcrl. Ent. Zeitschr. 46, p. 302 (1901).

perconstrictum Zerny, Iris 26, (1912) (ined.).

rufipes Rothsch., Nov. Zool. 18, p. 25 (1911).

simile Schaus, Proc. Zool. Soc. Lond. p. 225 (189t). Hamps., Cat. Lep. Phal. 1, p. 158, t. 6, f. 2 (1898).

sparta Druce, Ann. \& Mag. Nat. Hist. (7) 5, p. 507 (1900).

surrentum Druce, Proc. Zool. Soc. Lond. p. 375, t. 39, f. 8 (1883). - Hamps., Cat. Lop. Phal. 1, p. 160 (1898).

testaceum(-a) Walk., List Lep. Ins. Br. Mus. 1, p. 196 (1854) (Glaucopis). - Hamps., Cat. Lcp. Phal. 1, p. 158 , t. 6, f. $22(1898)$.

Trinitatis Rothsch., Nov. Zool. 18, p. 24 (1911).

vicinum Schrottky, Iris 24, p. 149 (1910).

Biologie: Schrottky, Iris 24, p. 149 (1910).

24

Homiuopsyehe Hamps.

Hamps., Cat. Lep. Phal. 1, p. 161 (1898).

ruatana Druce, Ann. \& Mag. Nat. Hist. (6) 2, p. 241 (1888) (Antichloris); Biol. Centr.-Am., Het. 2, p. 351, t. 72, f. 14 (1897). - Hamps., Cat. Lep. 1 Phal. 1, p. 161, f. 73 (1898).

Pompilopsis Hamps.

Hamps., Cat. Lep. Phal. 1, p. 161 (1898).

tarsalis Walk., List Lep. Ins. Br. Mus. 1, p. 196 (1854) (Qlaucopis). - Butl., Mllustr. Het. Br. Mus. 1, p. 28 , t. 13, f. 1 (1877). - Hamps., Cat. Lep. Phal. 1, p. 162, fo 74 (1898).

Guatemala, Panama

Ecuador

Amazonas

Bolivia

Colombia

Santa

Catharina, São Paulo

Paraguay

Bolivia

Colombia

Santa

Catharina

Venezuela

,

Colombia

Bolivia

Brit.-

Guayana,

Pará

Trinidad

Paraguay

Honduras

(I.Ruatan)

Mexico,

Guatemala,

Pará 
semihyalina Walk., List Lep. Ins. Br. Mus. 1, p. 197 (1854) Glaucopis). - Butl., Mlustr. Het. Br. Mus. 1, p. 28, t. 13, f. 9 (1877). - Druce, Biol. Centr.-Am., Hot. 1, p. 53 (1884); 2, p. 340 (1897).

vespiformis Herr.-Schäff., Aussereur. Schmett. 1, 1 p. 73, f. 425 (1855) (Pseudosphex).

\section{Pompiliodes Hamps.}

Hamps., Cat. Lep. Pral. 1, p. 162 (1898). A mycles Butl., Journ. Linn. Soc. Lond., Zool. 12, p. 369, t. 28, f. 18 (1876) (nec Herr.-Schäff.).

albomarginata Druce, Biol. Centr.-Am., Het. 1, p. 45, t. 7, f. 26 (1884) (Amycles). - Hamps., Cat. Lep. Phal. 1, p. 162, f. 75 (1898).

Biologie: Haase, Bibl. 'Zool. (2) 8, p. 77 (1893) (Mimicry).

aliena Walk., List Lep. Ins. Br. Mus. 1, p. 241 (1854) (Euchromia). - Hamps., Cat. Lep. Phal. 1, p. 163, f. 76 (1898).

flavifascia Herr.-Schäff., Aussereur. Schmett. 1, p. 73, f. 231 (1854) (Glaucopis). - Druce, Biol. Centr. Am., Het. 1, p. 45 (1884).

postiea Walk., List Lep. Ins. Br. Mus. 7, p. 1630 (1856) (Pampa). - Druce, Biol. Centr.-An., Het. 1, p. 45 (1884). - Hamps., Cat. Lep. Phal. 1, p. 163 , t. 6 , f. 6 (1898).

tenebrosa Walk., List Lep. Ins. Br. Mus. 1, p. 240 (1854) (Euchromia). - Hamps., Cat. Lep. Phal. 1, 4 p. 164, t. 6 , f. 26 (1898).

\section{Paramya Hamps.}

Hamps., Cat. Lep. Phal. 1, p. 164 (1898).

aeneta(-us) Schaus, Journ. New York Ent. Soc. 4, p. 131 (1896) (Chloropsinus). - Hamps., Cat. Lep. Phal. 1, p. 164, t. 6, f. 10 (1898).

ehrysonota Hamps., Cat. Lep. Phal. 1, p. 165, t. 7, f. 3 (1898).

llavia Schaus, Journ. New York Ent. Soc. 6, p. 139 (1898). - Hamps., Cat. Lep. Phal. 1, p. 165, f. 78 (1898),

intersecta Hamps., Cat. Lep. Phal. 1, p. 164, f. 77 (1898).

pleta Druce, Ann. \& Mag. Nat. Hist. (7) 1, p. 105 (1898). - Hamps., Cat. Lep. Phal, 1, p. 165, t. 6, f. $\theta$

Costarica,

Panama

\section{Guatemala, Venezuela, Pará, Amazonas, Rio Janeiro, Peru \\ Panama,}

Pará

Pará

Paraná

Pará

Paranḱ

Pará

Franz.-

Guayena 
Homoeocera Feld.

Feld., Reise Nov., Lep. Atlas p. 5 (1869). - But]., Journ. Linn. Soc. Lond., Zool. 12, p. 375, t. 27, f. 6 (1876). - Hamps., Cat. Lep. Phal. 1, p. 165 (1898). acuminata Walk., List Lep. Ins. Br. Mus. 7, p. 1608 (1856) (Lagaria). - Hamps., Cat. Lep. Phal. 1, p. 168 (1898). - Schrottky, Iris 15, p. 134 (1902).

leucostalacta Burm., Descr. Phys. Rep. Argent. Atlas p. 59 (1879) (Claucopis).

dalsa Schaus, Am. Lep. 1, p. 4, t. 1, f. 15 (1892) (Gartha).

bombiformis Rothsch., Nov. Zool. 18, p. 156 (1911).

crassa Feld., Reise Nov., Lep. 2, p. 5, t.- 102, f. 26 (1869).

- Druce, Biol. Centr.-Am., Het. 2, p. 339 (1897).

- Hamps., Cat. Lep. Phal. 1, p. 168, f. 81 (1898).

duronia Druce, Ann. \& Mag. Nat. Hist. (8) 7, p. 287 (1910).

Garleppi Rothsch., Nov. Zool. 18, p. 156 (1911).

gigantea Druce, Biol Centr.-Am. Het. 1, p. 54, t. 8, f. 5 (1884); 2, p. 341 (1897) (Gymnetia). - Hamps., Cat. Lep. Phal. 1, p. 166 (1898).

Iophocera Druce, Ann. \& Mag. Nat. Hist. (7) 1, p. 402 (1898). - Hamps., Cat. Lep. Phal. 1, p. 166, f. 79 (1898).

magnollmbata Dogn., Hét. nouv. Am. Sud 2, p. 3 (1911).

rhodocera Schaus., Trans. Am. Ent. Soc. 30, p. 135 (1904).

Rodriguezi Druce, Proc. Zool. Soc. Lond. p. 494 (1890); Biol. Centr.-Am., Het. 2, p. 340, t. 71, f. 5 (1897). - Hamps., Cat. Lep. Phal. 1, p. 168, f. 80 (1898).

sahacon Druce, Ann. \& Mag. Nat. Hist. (6) 18, p. 29 (1896); Biol. Centr.-Am., Het, 2, p. 340, t. 71 , f. 8 (1897). - Hamps., Cat. Lep. Phal. 1, p. 167 (1898).

sandion Druce, Ann. \& Mag. Nat. Hist. (8) 7, p. 287 (1910).

stictosoma Druce, Ann. \& Mag. Nat. Hist. (7) 1, p. 402 (1898). - Hamps., Cat. Lep. Phal. 1, p. 167, t. 6, f. 17 (1898).

tolosa Druce, Proo. Zool. Soc. Lond. p. 374 (1883) (Isanthrene). - Hamps., Cat. Lep. Phal. 1, p. 167, t. 6 , f. 28 (1898).

trizona Dogn., Ann. Soc. Eist. Beig. 50, p. 178 (1906). Watkinsi Druce, Ann. Mag. Nat. Hist. (8) 8, p. 716 16 (1911).

Paraná,
Santa
Catharina,
Argentinien

Peru

Costarica, Colombia

Golombia

Peru

Guatemala, Costarica

Brasilien (ubi?)

Franz.-

Guayana

Panama

Mexico, Guatemala

Panamia

Colombia

Colombia, Panama, (M.C.Vind.)

Ecuador

Venezuela

Peru

\section{Neotrichura Druce.}

Druce, Ann. \& Mag. Nat. Hist. (6) 18, p. 33 (1896); Biol. Centr. Am., Het. 2 , p. 350 (1897).- Hamps., Cat. Lep. Phal. 1, p. 169 (1898).

penates Druce, Ann. \& Mag. Nat. Hist. (6) 18, p. 33 (1896); Biol. Centr.-Aw., Het. 2, p. 350, t. 72 , f. 10 (1897). - Hamps., Cat. Lep. Phal. 1, p. 169, f. 82 (1898).

$\because$ dukinfieldia Schaus, Journ. New York Ent. 1 Soo. 4, p. 132 (1897) (Cosmasoma).

Panama, São Paulo (M.C.Vind.), Rio Janeiro, Paraná, Paraguay 


\section{Isanthrene Hübn.}

Hübn., Verz. bek. Schmett. p. 125 (1826). - Butl., Journ. Linn. Soc. Lond., Zool. 12, p. 374, t. 27, f. 4 (1876). - Hamps., Cat. Lep. Phal. 1, p. 170 (1898).

Biologie: Haase, Bibl. Zool. (2) 8, p. 76 (1893) (Mimicry).

a terrima Walk., List Lep. Ins. Br. Mus. 31, p. 85 (1864) Ecuador, (Gymnelia). - Hamps., Cat. Lep. Phal. 1, Amazonas p. 176 , t. 6 , f. 21 (1898).

phyleis Druce, Proc. Zool. Soc. Lond. p. 375 (1883) (Erruca).

azia Druce, Biol. Centr.-Am., Het. 1, p. 53, t. 6, f. 21 (1884); 2, p. 340 (1897) (Erruca). - Hamps., Cat. Jep. Phal. 1, p. 175 (1898).

basifera Walk., List Lep. Ins. Br. Mus. 31, p. 86 (1864). - Hamps., Cat. Lep. Phal. 1, p. 176 , t. 6, f. 3 (1898).

grenadensis Butl., Journ. Linn. Soc. Lond., Zool. 12, p. 378 (1876) (Erruca).

Cajetani Rothsch., Nov. Zool. 18, p. 26 (1911).

Champloni Druce, Biol. Centr.-Am., Het. 1, p. 50, t. 8, f. 6 (1884); 2, p. 339 (1897). - Hamps., Cat. Lep. Phal. 1, p. 174 (1898).

columbiana Rothsch., Nov. Zool. 18, p. 26 (1911).

crabroniformis Stdgr., Verh. zool.-bot. Ges. Wien 25, p. 120 (1875). - Druce, Biol. Centr.-Am., Het. 1, p. 51, t. 6, f. 23 (1884). - Hamps., Cat. Lop. Phal. 1, p. 172 (1898).

Biologie: Haase, Bibl. Zool. (2) 8, p. 76 (1893) (Mimicry).

Drucei Rothsch., Nov. Zool. 18, p. 26 (1911).

echemon Druce, Biol. Centr.-Am., Het. 1, p. 50, t. 6 , f. 22 (1884). - Hamps., Cat. Lep. Phal. 1, p. 174 (1898).

Felderi Druce, Proc. Zool. Soc. Lond. p. 377, t. 39, f. 9 (1883) (Dycladia); Biol. Centr.-Am., Het. 1, p. 63 , t. 7 , f. 18 (1884); 2, p. 348 (1897). Hamps., Cat. Lep. Phal. 1, p. 170, f. 83 (1898).

fulvipuneta Hamps., Ann. \& Mag. Nat. Hist. (7) 15, p. 427 (1905)

varia Hamps., Cat. Lep. Phal. 1, p. 176 (1898) (pr. p.).

incendiaria Hübn., Samml. Exot. Schmett. 1, t. 166 (1827) (Glaucopis). - Meig., Syst. Bearb. Eur. Schmett. 3, praefatio, t. 120, f. 9 (1832). Hamps., Cat. Lep. Phal. 1, p. 174 (1898). Silva, Lep. Bras. p. 127, t. 23, f. 80 (1907).

ab. maxima Butl., Journ. Jinn. Soc. Lond., Zool. 12, p. 375 (1876), - Hamps., Cat. Lep. Phal. 1, p. 175 (1898).

Biologie: Haasc, Bibl. Zool. (2) 8, p. 76 (1893) (Mimicry).

joda Druce, Ann. \& Mag. Nat. Hist. (7) 11, p. 196 (1903).

Mathani Rothsch., Ann. \& Mag. Nat. Hist. (8) 5, p. 511

Mexico,

Guaternala

Colombia

Colombia

Zentral-

amerika

(ubi ?)

Panama

Colombia

Costarica,

Panama,

Colombia,

Venezuelı

Colombia

Guatemala

Mexico,

Guatemala,

Costarica,

Ecuador

Panama

Rio Janeiro (1910).

Peru

Colombia 
melaena (melas) Cram., Pap. Exot. 1, p. 71, t. 45, f. G (1775) (Sphinx). - Hamps., Cat. Lep. Phal. 1, p. 173 , f. 84 (1898).

flavicornis Fabr., Mant. Ins. 2, p. 104 (1787) (Zygaena).

vespoides Walk., List Lep. Ins. Br. Mus. 1, p. 155 (1854) (Glaucopis).

Biologie: Haaso, Bibl. Zool. (2) 8, p. 76 (1893) (Mimicry).

minor Butl., Journ. Linn. Soc. Lond., Zool. 12, p. 395 (1876) (Dycladia). - Hamps., Cat. Lep. Phal. 1, p. 178, t. 6, f. 20 (1898).

brom $u s$ var. Walk., List Lep. Ins. Br. Mus. 1, p. 179 (1854) (Glaucopis).

monticola Schaus, Ann. \& Mag. Nat. Hist. (8) 7, p. 173 (1911).

notipennis Butl., Journ. Linn. Soc. Lond., Zool. 12, p. 378 (1876) (Erruca). - Hamps., Cat. Lep. Phal. 1, p. 177, t. 6, f. 23 (1898).

pelor Druce, Ann. \& Mag. Nat. Hist. (6) 20, p. 302 (1897) (Homoeocera). - Hamps., Cat. Lep. Phal. 1, p. 175 , t. 6 , f. 18 (1898).

pentagona Schaus, Journ. Now York Ent. Soc. 6, p. 139 (1898). - Hamps., Cat. Lep. Phal. 1, p. 170, t. 6, f. 19 (1898).

Perboscl(-ii) Guér., Icon. Régno Animal, Ins. p. 501 (1843) (Glaucopis). - Druce, Biol. Centr.-Am., Het. 2, p. 339 (1897). - Hamps., Cat. Lep. Phal. 1, p. 173 (1818).

pompiloides Walk., List Lop. Ins. Br. Mus. 1, p. 156 (1854) (Glaucopis). - Butl., Illustr. Het. Br. Mus. 1, p. 26, t. 12, f. 10 (1877). Druce, Biol. Centr.-Am., Het. 1, p. 50 (1884); 2, p. 339 (1897).

Pertyl Herr.-Schäff., Aussereur. Schmett. 1, p. 73, f. 249 (1854) (Glancopis). - Neum. \& Dyar, Journ. New York Ent. Soc. 1, p. 107 (1893). - Hamps., Cat. Lep. Phal. 1, p. 175 (1898).

porphyria Walk., List Lep. Ins. Br. Mus. 1, p. 157 (1854) (Glancopis). - Hamps., Cat. Lep. Phal. 1, p. 178 (1898).

machile Herr.-Schäff., Aussereur. Schmett. 1, p. 73, f. 245 (1854) (Glaucopis).

profusa Hamps., Cat. Lep. Phal. 1, p. 177, t. 6, f. 5 (1898).

pyrocera Hamps., Cat. Lep. Phal. 1, p. 173, t. 6, f. 16 (1898)

incendiaria Druce, Biol. Centr.-Am., Het. 1, p. 50 (1884) (nec Huibn.).

Schausi Rothsch., Nov. Zool. 18, p. 25 (1911).

thyestes Druce, Proc. Zool. Soc. Lond. p. 374, t. 39, f. 4 (1883). - Hamps., Cat. Lep. Phal. 1, p. 172 (1898).

Tryhanei Rothsch., Nov. Zool. 18, p. 26 (1911).

ustrina Hübn., Zutr. Exot. Schmett. 3, p. 25, f. 493,494 (1827). - Hamps., Cat. Lep. Phal. 1, p. 172 (1898).

Surinam, Pará, Amazonas, Paraguay

Brasilien (ubi?)

Costarica

Amazonas

Eouador

Peru

Mexico,

Guatemala

Espiritu

Santo

(Georgia

ex err.)

Surinam,

Pará,

Amazonas

Amazonas,

São Paulu

Mexico

Ecuador

Trinidad

Cuba, São

Paulo (M.

C. Vind.) 
varia Walk., List Lep. Ins. Br. Mus. 1, p. 157 (1854) (Glaucopis). - Druce, Biol. Centr.-Am., Het. 1, p. 52 (1884). - Hamps., Cat. Lep. Phal. 1, p. 176 , t. 6 , f. 1 (1898).

vespiformis Butl., Journ. Linn. Soo. Lond., Zool. 12, p. 379 (1876) (Erruca). - Hamps., Cat. Lep. 31 Phal. 1, p. 177, t. 6, f. 25 (1898).

Panama,

Pará, Rio Janeiro (M. C. Vind.)

Amazonas

\section{Fyda Walk.}

Walk., list Lep. Ins. Br. Mus. 1, p. 181 (1854). - Hamps., Cat. Lep. Phal. 1, p. 178 (1898).

basilutea Walk., List Lep. Ins. Br. Mus. 1, p. 182 (1854) (Glaucopis). - Hamps., Cat. Lep. Phal. 1, p. 178 , f. 85 (1898).

xanthor rhina Herr.-Schäff., Aussereur. Schmett. 1, p. 73, f. 226 (1854) (Glaucopis). - Butl., Illustr. 1

Het. Br. Mus. 1, p. 35, t. 11, f. 8 (1877).

Colombia, Venezuela, Pará, Pernambuco, Rio

Janeiro

\section{Autochloris Hübn.}

Hübn., Verz. bek. Schmett. p. 125 (1826). - Hamps., Ann. \& Mag. Nat. Hist. (7) 15, p. 427 (1905).

Bombiliodes Hamps., Cat. Lep. Phal. 1, p. 179 (1898).

almon Cram., Pap. Exot. 3, p. 52, t. 224, f. F (1779) (Sphinx). - Stoll, Suppl. Cram. p. 50, t. 11, f. Id (1787). - Sepp, Surin. Vlind. 3, p. 269, t. 123 (1848). - Hamps., Ann. \& Mag. Nat. Hist. (7) 15 , p. $427(1905)$.

Biologie: Stoll, Suppl. Cram. p. 50, t. 11, f. 1, 1A, IB (1787). - Sepp, Surin. Vlind. 3, p. 269, t. 123 (1848).

aroa Schaus, Proc. Zool. Soc. Lond. p. 225 (1894) (Gymnelia ). - Hamps., Cat. Lep. Phal. 1, p. 182, t. 7 , f. 8 (1898).

bijuneta Walk., List Lep. Ins. Br. Mus. 7, p. 1602 (1856) (Gymnelia). - Hamps., Cat. Lep. Phal. 1, p. 181, t. 7, f. 1 (1898).

consociata Walk., List Lep. Ins. Br. Mus. 31, p. 85 (1864) (Gymnelia).

capistrata Fabr., Syst. Ent. p. 554 (1775) (Zygaena). - Hamps., Cat. Lep. Phal. 1, p. 180 (1898).

selecta Herr.-Schäff., Aussereur. Schmett. 1, p. 73, f. 256 (1854) (Glaucopis).

caruinata Hamps., Ann. \& Mag. Nat. Hist. (7) 7, p. 246 (1901) (Bombiliodes).

caunus Cram., Pap. Exot. 3, t. 224, f. E (1779) (Sphinx). - Hamps., Cat. Lep. Phal. 1, p. 181 (1898).

cincta Schaus, Proc. U. S. Nat. Mus. 29, p. 184 (1905) (Bombiliodes).

Surinam

Venezuela

Amazonas

Brasilien

(ubi?)

Bahama-I.

(Nassau)

Surinam,

São Paulo

Franz.-

Guayana

collocata Walk., List Lep. Ins. Br. Mus. 31, p. 84 (1864)

(Gymnelia). - Hamps., Cat. Lep. Phal. 1, p. 184,

Amazonas t. 7. f. 22 (1898). 
completa Walk., List Lep. Ins. Br. Mus. 1, p. 153 (1854) (Glaucopis). - Butl., 1llustr. Het. Br. Mus. 1, p. 29 , t. 12, f. 3 (1877). - Hamps., Cat. Lep. Phal. 1, p. 186 (1S98).

cuma Druce, Ann. \& Mag. Nat. Hist. (6) 20, p. 302 (1897) (Gymnelia). - Hamps., Cat. Lep. Phal. 1, p. 181, t. 7 , f. $2(1898)$.

ectomelaena Hamps., Cat. Lel. Phal. 1, p. 182, t. 7 , f. 7 (1898) (Bombiliodes).

magrus Cram., Pap. Exot. 3, 1. 248, f. D (1780) (Sphinx). - Hamps., Cat. Lep. Phal. 1, p. $18 \dot{\text { (1898). }}$

ab. dexamene Druce, Ann. \& Mag. Nat. Hist. (6) 20, p. 302 (1897) (Gymnelia). - Hamps., Cat. Lep. Phal. 1, p. 185 (1898).

Biologic: Stoll, Suppl. p. 63, t. 13, f. 6 (1790). Jannaicensis Schaus, Journ. Now York Ent. Soc. 9, p. 40 (1901) (Bombiliodes).

Jansonis Butl., Cist. Ent. 1, p. 116 (1872) (Gymnelia); Lep. Exot. p. 175, t. 61, f. 17 (1874). - Druce, Biol. Centr.-Am., Het. 1, p. ól (1884). - Hamps., Cat. Lep. Phal. 1, p. 180 (1898).

laennus Walk., List Lep. Ins. Br. Mus. 1, p. 154 (1854) (Glaucopis). - Butl., Illustr. Het. Br. Mus. 1, p. 28, t. 12, f. 8 (1877). - Hamps., Cat. Lep. Phal. 1, p. 183, f. 86 (1898).

Mathani Rothsch., Nov. Zool. 18, p. 27 (1911).

patagiata Dyar, Proc. Ent. Soc. Wash. 11, p. 19 (1909). gerra Schaus, Proc. Zool. Soc. Lond. p. $27 \pm(1892)$ (Gymnelia); Am. Lep. p. 3, t. 1, f. 10 (1892). - Hanps., Cat. Lep. Phal. 1, p. 184 (1898).

simplex Walk., List Lop. Ins. Br. Mus. 7, p. 1603 (1856) (Gymnelia). - Hamps., Cat. Lep. Phal. 1, p. 183 , t. 7, f. 21 (1898).

simulans Druce, Ann. \& Mag. Nat. Hist. (8) 3, p. 457 (1909) (Bombiliodes).

Whitelyi Druce, Proc. Zool. Soc. Lond. p. 376 (1883) (Gymnelia). - Hamps., Cat. Lep. Phal. 1, p. 184, t. 7, f. 28 (1898).

xantlogastroides Schaus, Journ. New York Ent. Soc. 9, p. 40 (1901) (Bombiliodes).

xenodorus Druce, Biol. Centr.-Am., Het. 1, p. 55, t. 7 , f. 1 (1884); 2, p. 341 (1897) (Gymnelia). 23 Hamps., Cat. Lep. Phal. 1, p. 182 (1898).

Pará

Colombia, . Bolivia

São Pavilo

Surinam, Amazonas

Jamaica

Costarica, Panama, Colombia

Rio Janciro

Colombia

Mexico

Rio Janeiro

Pará

Franz. -

Peru

Guayana

Paraná

Mexico, Guatemala

\section{Sarosa Walk.}

Walk., List Lep. Ins. Br. Mus. 1, p. 151 (1854). - Butl., Journ. Linn. Soc. Lond., Zool. 12, p. 377, t. 27, f. 11 (1876). - Hamps., Cat. Lep. Phal. 1, p. 185 (1898).

acutior Feld., Reise Nov., Lep. 2, p. 6, t. 102, f.27 (1.869) (Isanthrene). - Hamps., Cat. Lep. Phal. 1, p. 187 (1898).

Boenninghauseni Rothsch., Nov. Zool. 18, p. 27 (1911). eounotata Hamps., Ann. \& Mag. Nat. Hist. (7) 8, p. 171 (1901).

epona Dogn., Ann. Soc. Ent. Belg. 46, p. 226 (1902). Venezuela

Ecuador, Amazonas

? Rio Janeiro Peru 
helotes Druce, Ann. \& Marr. Nint. Hist. (7) 5, p. 507 (1900).

? lgnicolor Mén., Cat. Lép. Pétersb. 2, p. 139, t. 14, f. 3 (1857) (Laemocharis). - Hamps., Cat. Lep. Phal. 1, p. 185 (1898) (huius generis?).

Klagesi Rothsch., Nov. Zool. 18, p. 27 (1911).

? leuce Maassen in Stübel's Reise S. Am., Lep. p. 129 , t. 4, f. 18 (1890) (Dasysphin $x$ ). - Hamps., Cat. Lcp. Phal. 1, p. 188 (1898) (huius generis?).

lutibusis Hamps., Ann. \& Mag. Nat. Hist. (7) 8, p. 171 (1901).

meridensis Schaus, Trans. Am. Ent. Soc. 30, p. 135 (1904). moru Schaus, Ann. \& Mag. Nat. Hist. (8) 7, p. 173 (1911). notata Butl., Journ. Iinn. Soc. Lond., Zool. 12, p. 390 (1876) (Ilipa). - Hamps., Cat. Lep. Phal. 1, p. 186, f. 87 (1898).

ufentina Druce, Proc. Zool. Soc. Lond. p. 377 (1883) (Cosmasoma).

ozora Druce, Proc. Zool. Soc. Lond. p. 374, t. 39 , f. 6 (1883) (Homococera). - Hamps., Cat. Lep. Phal. 1, p. 187 (1898).

pompilina Butl., Journ. Linn. Soc. Lond., Zool. 12, p. 377 (1876). - Druce, Biol. Centr.-Am., Het. 1, p. 52, t. 6, f. 24 (1884). - Hamps., Cat. Lep. Phal. 1, p. 187 (1898).

seslifornis Walk., List Lep. Ins. Br. Mus. 1, p. 151 (1854) (Glaucopis). - Butl., Illustr. Het. Br. Mus. 1 , p. 27 , t. 12 , f. 5 (1877). - Hamps., Cat. Lep. Phal. 1, p. 185 (1898).

xauthobasis Druce, Ann. \& Mag. Nat. Hist. (7) 1, p. 403 (1898). - Hamps., Cat. Lep. Phal. 1, p. 186, t. 7 , f. 13 (1898).

xanthotarsis Lathy, The Ent. 32, p. 116 (1899). 17

Colombia

Minas Geraës

Venezuela

Colombia

Panama

Venezuela

Costarica

Colombien,

Bolivien

Colombien

Panama, Ecuador, EspirituSanto

Venezuela

Ecuador

Venezuela

\section{Dasysphinx Feld.}

Feld., Reise Nov., Lep. Atlas p. 3 (1869). - Boisd., Lép. Hét. 1, p. 370 (1875). - Hamps., Cat. Lep. Phal. 1, p. 188 (1898).

mugescens Feld., Reise Nov., Lep. 2, p. 3, t. 102, f. 25

Colombia (1869). - Hamps., Cat. I.ep. Phal. 1, p. 188 f. 88 (1898).

volatilis Schaus, Ann. \& Mag. Nat. Hist. (8) 6, p. 189 Costarica 2 (1910).

\section{Lasiosceles Hamps.}

Hamps., Ann. \& Mag. Nat. Hist. (8) 4, p. 345 (1909).

erythrozonata Hamps, Ann. \& Mag. Nat. Hist. (8) 4, Panama 1 p. 345 (1909).

\section{Gymnelia Walk.}

Walk., List Lep. Ins. Br. Mus. 1, p. 152 (1854). - Butl., Journ. Linn. Soc. Lond., Zool. 12, p. 382, t. 27, f. 13 (1876). - Hamps., Cat. Lep. Phal. 1, p. 189 (1898).

Andrenimorpha Butl., Journ. Linn. Soc. Lond. Zool. 12, p. 382 , t. 27 , f. $10(1876)$. 
IEnioche Druce, Ann. \& Mag. Nat. Hist. (6) 18, p. 34 (1896) (an= Pseudosphenopiera Butl.?).

? Sansaptera Schaus, Journ. New York Ent. Soc. 4, p. 132 (1896). (an = Pseudosphenopiera Butl. ?).

Biologie: Seitz, Stett. Ent. Zeitg. 51, p. 262 (1890) (Mimicry). Baroni Rothsch., Ann. \& Mag. Nat. Hist. (8) 5, p. 510 Ecuador (1910).

Boettgeri Rothsch., Nov. Zool. 18, p. 28 (1911).

Peru

Buckleyi Druce, Proc. Zool. Soc. Lond. p. 374, t. 39, f. 5 (1883) (Homoencera). - Hamps., Cat. Lep. Phal. 1, p. 192 (1898).

chimsera Rothsch., Nov. Zool. 18, p. 27 (1911).

cocho Schaus, Journ. Now York Ent. Soc. 4, p. 132 (1896) (Sansapiera). - Hamps., Cat. Lep. Phal. 1, p. 189 , t. 7 , f. 6 (1898); Ann. \& Mag. Nat. Hist. (7) 15, p. 426 (1905) (ad Pseudosphenoplera Butl. ponenda?).

Doneasteri Rothsch., Nov. Zool. 18, p. 28 (1911).

flavicornis Druce, Proc. Zool. Soc. Lond. p. 373 , t. 39, f. 3 (1883) (Calonolos). - Hamps., Cat. Lep. Phal. 1, p. 193 (1898).

gemmifera Walk., List Lep. Ins. Br. Mus. 1, p. 152 (1854) (Glaucopis). - But., Mlustr. Het. Br. Nlus. 1, p. 27, t. 12, f. 12 (1877). - Hamps., Cat. Lop. Phal. 1, p. 191 (1898).

herodes Druce, Proc. Zool. Soc. Lond. p. 372 , t. 39, f. I (1883) (Eupyra). - Hamps., Cat. Lep. Phal. 1, p. 193 (1898).

Jordaui Zerny nom. nov.

Baroni Rotisch., Nov. Zool. 18, p. 28 (1911) (nec Rothsch. 1910).

intimarginata Hamps., Cat. Lep. Phal. 1, p. 191, t. 7, f. 27 (1898).

ifrcen Druce, Proc. Zool. Soc. Lond. p. 375 (1883) (Homoeocera). - Hamps., Cat. Lep. Phal. 1, p. 190 t. 7 , f. 9 (1898).

Ockendenf Rothsch., Ann. \& Mag. Nat. Hist. (8) 5, p. 510 (1910).

parapanema Dogn., Hét. nouv. Am. Sud 2, p. 4 (1911). peralea Dogn., Hét. nouv. Am. Sud 1, p. 3 (1910).

pilosa Rothsch., Ann. \& Mag. Nat. Hist. (8) 5, p. 511 (1910).

pitthea Druce, Ann. \& Mag. Nat. Hist. (6) 18, p. 34 (1896) (Enioche). — Hamps., Cat. Lep. Phal. 1, p. 189, f. 89 (1898); Ann \& Mag. Nat. Hist. (7) 15 , p. 426 (1905) (an $=$ Pserdosphenopiera basalis Wilk.?).

piagiata Rothsch., Nov. Zool. 18, p. 156 (1911).

piumosa Rothsch., Nov. Zool. 18, p. 156 (1911).

taos Hamps., Cat. Lep. Phal. 1, p. 191, t. 7, f. 10 (1898). tarsipuneta Schaus, Proc. U. S. Nat. Nus. 29, p. 184 (1905).

torquata(-us) Druce, Proc. Zool. Soc. Lond. p. 377 (1883). Hamps., Cat. Lep. Phal. 1, p. 192, t. 7, f. 16 (1898).

xanthogastra Perty, Delcct. Anim. Artic. p. 156, t. 31, f. 5 (1834) (Glaucopis). - Herr.-Schäff., Aussereur. Schmett. 1, f. 298 (1854). - Hamps., Cat. Lep. Phal. 1, p. 191, f. 90 (1898).

Pcru

Peru,

São Paulo

Venezuela

Colombia

Venezuela

Ecuador

Ecuador, Peru

Colombia

Ecuador

Poru

São Paulo Colombia

Poru

,

Colombia

Santa"

Catharina

Rio Janeiro

Rio Janeiro, Santa Catharina (M. C. Vind.), Paraguay, Argentinien 
Mallodeta Butl.

Butl., Journ. Linn. Soc. Lond., Zool. 12, p. 398 (1876). - Hamps., Cat. Lep. Phal. 1, p. 193

Lycorea Walk., List. Lop. Ins. Br. Jlus. 1, p. 192 (1854) (neo Dbld. 1846).

ciarata Walk., List Lep. Ins. Br. Mus. 1, p. 192 (1854) (Glaucopis). - Hamps., Cat. Lep. Phal. 1, p. 195,

t. 7, f. 5 (1898).
consors Walk., List Lep. Ins. Br. Mus. 1, p. 183 (1854)

(Glaucopis). - Hamps., Cat. Lep. Phal. 1, p. 194, f. 91 (1898).

norma Herr.-Schäff., Aussereur. Schmett. 1, p. 73, f. $251 \mathrm{~b}$ (1854) (Glaucopis).

parthenii Fabr., Syst. Ent. 3, p. 402 (1793) (Zygaena). - Hamps., Cat. Lep. Phal. 1, p. 195, f. 92 (1898).

multicincta Walk., List Lep. Ins. Br. Mus. 1, p. 163 (1854) (Glaucopis). - Butl., Illustr. Het. Br. Mus. 1, p. 35, t. 11, f. 11 (1877).

sanguipuncta Druce, Ann. \& Mag. Nat. Hist. (7) 1, p. 403 (1898). - Hamps., Cat. Lep. Phal. 1, p. 194 , t. 8 , f. 13 (1898).

sortita Walk., List Lep. Ins. Br. Mus. 1, p. 184 (1854) (Glaucopis). - Hamps., Cat. Lcp. Phal. 1, p. 194 (1898).

Deyrollę Herr.-Schäff., Aussereur. Schmett. 1, p. 73, f. 247, 25la (1854) (Glaucopis) (nec Walk.). a ecyra Herr.-Schäff., Aussereur. Schmett. 1, p. 73, f. 250 (1854) (Glaucopis). - Butl., Illustr. Het. 5 Br. Mus. 1, p. 36, t. 13, f. 6 (1877).

\section{Phoenicoprocta Hamps.}

Hamps., Cat. Lep. Phal. 1, p. 196 (1898).

Hyela Walk., List Lep. Ins. Br. Mus. 1, p. 172 (1854) (neo Steph. 1850).

anaiis Schrottky, Iris 22, p. 123 (1909).

astrifera Butl., Illustr. Het. Br. Mus. 1, p. 38, t. 17, f. 2

(1877) (Iyela). - Hamps., Cat. Lep. Phal. 1, p. 199 (1898).

Baeri Rothsch., Nov. Zool. 18, p. 28 (1911).

Chamboni Dogn., Ann. Soc. Ent. Belg. 46, p. 473 (1902).

chrysorrhoen Hamps., Cat. Lop. Phal. 1, p. 196, f. 93 (1898).

cubana Druce, Ann. \& Mag. Nat. Hist. (7) 3, p. 228 (1899).

eximia Herr.-Schäff., Corresp.-Bl. min.-zool. Ver. Regensb. 20, p. 115 (1866) (Glaucopis). - Hamps., Cat. Lep. Phal. 1, p. 199, t. 17, f. 15 (1898).

fiavipicta Hamps., Ann. \& Mag. Nat. Hist. (8) 4, p. 345 (1909).

haemorrhoidalis Fabr., Syst. Ent. p. 549 (1775) (Sesia). - Hamps., Cat. Lep. Phal. 1, p. 197, t. 7, f. 14 (1898).

frontalis Walk, List Lep. Ins. Br. Mus. 1, p. 188 (1854) (Glaucopis).

Brasilien

(ubi?)

Amazonas (M. C. Vind.), São Paulo, Santa Catharina

Haiti (M.C.Vind.)

Paraguay

Rio Janeiro,

Santa

Catharina

(M.C.Vind.)

Paraguay

Amazonas

Goyaz

San Salvador

Colombia

Cuba

Cuba,

Portorico,

Sta. Cruz

Brit.-

Guayana

Brasilien

(ubi?) 
lydia Druce, Ann. \& Mag. Nat. Hist. (6) 4, p. 84 (1889) (Dycladia); Biol. Centr.-Am., Het. 2, p. 346, t. 71, f. 25 (1897). - Hamps., Cat. Lep. Phal. 1, p. 198 (1898).

mexicana Walk., List Lep. Ins. Br. Mus. 31, p. 84 (1864) (Gymnelia). - Druce, Biol. Centr.-Am., Het. 1, p. 61 , t. 7, f. 2 (1884); 2 , p. 346 (1897). - Hamps., Cat. Lep. Phal. 1, p. 200 (1898).

metachrysea Druce, Ann. \& Mag. Nat. Hist. (7) 1, p. 404 (1898). - Hamps., Cat. Lcp. Phal. 1, p. 196, t. 7 , f. 12 (1898).

nigrivalvata Dogn., Hét. nouv. Am. Sud 2, p. 4 (1911).

rubriventris(-er) Hamps., Cat. Lop. Phal. 1, p. 198, f. 94 (1898).

sa.nguinea Druce, Biol. Centr.-Am., Het. 1, p. 63 (1881) (Hyela) (nec Walk.).

sanguinen Walk., List Lep. Ins. Br. Mus. 1, p. 172 (1854) (Glaucopis). - Butl., Illustr. Het. Br. Mus. 1, p. 37, t. 11, f. 13 (1877). - Hamps., Cat. Lep. Phal. 1, p. 197 (1898).

Steinbachi Rothsch., Nov. Zool. 18, p. 29 (1911).

teda Walk., List Lep. Ins. Br. Mus. 1, p. 177 (1854) (Glaucopis). - Butl., Illustr. Het. Br. Mus. 1, p. 34, t. 11, f. 1 (1877). - Hamps., Cat. Lep. Phal. 1, p. 200 (1898).

thern Druce, Anu. \& Mag. Nat. Hist. (6) 4, p. 85 (1889) (Dycladia): Biol. Centr.-Am., Hct. 2, p. 347, t. 71, f. 26 (1897). — Hamps., Cat. Lep. Phal. 1, p. 199 (1898).

vacillaus Walk., List Lep. Ins. Br. Mus. 7, p. 1617 (1856) (Eunomia). - Hamps., Cat. Lep. Phal. 1, p. 197 , t. 7 , f. 11 (1898).
Mexico

Mexico

Franz.:

Guayana .

Argentinien

Panama

Honduras

Bolivia

Santa

Catharina,

São Paulo

(M. C.

Vind.)

Mexico

Venezuela, Colombia, São Paulo, Santa Catharina

\section{Eunomia Hübn.}

Hübn., Verz. bek. Schmett. p. 125 (1826). - Hamps., Cat. Lep. Phal. 1, p. $200(1898)$.

Marissä Walk., List Lep. Ins. Br. Mus. 1, p. 174 (1854).

caymanensis Hamps., Ann. \& Mag. Nat. Hist. (8) 8, p. 395 (1911).

columbina (colombina) Fabr., Syst. Ent. (1) 3, p. 403 (1793) (Zygaena). - Hübn., Zutr. Exot. Schmett. 1, p. 8, f. 9, 10 (1827). - Butl., Tllustr. Het. Br. Mus. 1, p. 34, t. 11, f. 3 (1877). - Hamps., Cat. Lep. Phal. 1, p. 201 , f. 95 (1898).

fasciatelìa Mén., Cat. Lép. Pćtersb. 2, p. 140, t. 14, f. 4 (1857) (Laemocharis).

insularis Grote, Proc. Ent. Soc. Philad. 6, p. 188, t. 5, f. 5 (1866). - Hamps., Cat. Lep. Phal. 1, p. 202 (1898).

elegantula Herr.-Schäff., Corresp.-Bl. min.-zool.

I. Grand

Cayman b. Cuba

Haiti, Honduras, Brasilen (ubi?) Ver. Regensb. 20, p. 114 (1866) (Glaucopis).

Cuba 
latenigra Butl, Journ. Linn. Soc. Lond., Zool. 12, p. 395 (1876) (Marissa). - Hamps., Cat. Lep. Phal. 1, p. 202 , t. 7 , f. 18 (1898).

? nitidula Herr.-Schäff., Corresp.-Bl. min.-zool. Ver. Regensb. 20, p. 114 (1866) (Glaucopis). - Hamps., Cat. Lep. Phal. 1, p. 202 (1898) (huius generis?). rubripunctata Butl., Journ. Linn. Soc. Lond., Zool. 12, p. 395 (1876) (Marissa). - Hamps., Cat. Lep. 6 Phal. 1, p. 201, t. 7, f. 17 (1898).

Bahama-I., Honduras

Cuba

Jamaica

\section{Phia Walk.}

(Pheia) Walk., List Lep. Ins. Br. Mus. 1, p. 146 (1854). - Hamps., Cat. Lep. Phal. 1, p. 203 (1898).

albisigna Walk., List Lep. Ins. Br. Mus. 1, p. 146 (1854) (Glaucopis). - Butl., Mlustr. Het. Br. Mus. 1, p. 30, t. 7, f. 14 (1877). - Druce, Biol. Centr. -Am. Het. 2, p. 343 (1897). - Hamps., Cat. Lep. Phal. 1, p. 205, f. 97 (1898).

bisigna Kaye, The Ent. 44, p. 146 (1911).

costalis Rothsch., Nov. Zool. 18, p. 29 (1911)

daphaena Hamps., Trans. Ent. Soc. Lond. p. 241 (1898); Cat. Lep. Phal. 1, p. 205, t. 7, f. 25 (1898).

discophora Dogn., Ann. Soc. Ent. Belg. 53, p. 213 (1909). elegans Druce, Biol. Centr.-Am., Het. 1, p. 59, t. 7, f. 13 (1884) (Cosmosoma). - Hamps., Cat. Iep. Phal. 1, p. 203 (1898).

Drucei Kirby, Cat. Het. 1, p. 140 (1892) (Cosmo. soma). - Druce, Biol. Centr.-Am., Het. 2, p. 344 (1897).

cingla Schaus, Proc. Zool. Soc. Lond. p. 227 (1894) (Cosmosoma).

fluviclneta Dogn., Ann. Soc. Ent. Belg. 50, p. 178 (1906).

gaudens Walk., List Lep. Ins. Br. Mus. 7, p. 1607 (1856) (Poecilosoma). - Hamps., Cat. Lep. Phal. 1, p. 203 , f. 96 (1898).

haemoperu (haemapera) Schaus, Journ. New York Ent. Suc. 6, p. 139 (1898). - Hamps., Cat. Lep. Phal. 1, p. 204, t. 7, f. 24 (1898).

haematosticta Jones, Trans. Ent. Soc. Lond. p. 143 (1908).

lateralls Klages, Proc. U. S. Nat. Mus. 29, p. 533 (1906). pyrama Dogn., Hét. nouv. Am. Sud 3, p. 3 (1911)

spcruns Walk., List Lep. Ins. Br. Mus. 7, p. 1606 (1856) (Poccilosoma). - Hamps., Cat. Lep. Phal. 1, p. 204, t. 7, f. 23 (1898).

uflca Druce, Ann. \& Mag. Nat. Hist. (6) 4, p. 85 (1889) (Dycladia); Biol. Centr.-Am. Het. 2, p. 348, t. 71 , f. 28 (1897). - Hamps., Cat. Lep. Phal. 1, p. 205 (1898).

ranthozona Dogn., Hét. nouv. Am. Sud 1, p. 3 (1910).

Honduras, Venezuela Amazonas

Brit.-

Guayana

Bolivia

Dominica, Santa Lucia

Colombia

Mexico, Guatemala, Costarica, Panama, Venezuela

Argentinien

Venezuela, Pará

Santa Catharina

Paraná

Venezuela

Franz.Guayana

Colombia, Amazonas

Mexico, Venezuela

Franz.Guayana 
IImagyra Hamps.

Hamps., Cat. Lep. Phal. 1, p. 206 (1898).

pampa Druce, Proc. Zool. Soc. Lond. p. 284 (1893) (Metastatia ). - Hamps., Cat. Lep. Phal. 1, p. 206, f. 98 (1898).

pulchella Klages, Proc. U. S. Nat. Mus. 29, p. 534 (1906). Venezuela 2

\section{Loxophlebia Butl.}

Butl., Journ. Linn. Soc. Lond., Zool. 12, p. 381, t. 27, f. 14 (1876). Hamps., Cat. Lop. Phal. 1, p. 206 (1898).

albicincta Dogn., Ann. Soc. Ent. Belg. 51, p. 223 (1907). asmodeus Druce, Proc. Zool. Soc. Lond. p. 378 (1883) (Desmidocnemis). - Hamps., Cat. Lep. Phal. 1, p. 211, t. 7 , f. 26 (1898).

aurantiaca Jones, Trans. Ent. Soc. Lond. p. 143 (1908).

broteas Schaus, Proc. Zool. Soo. Lond. p. 276 (1892) (Dycladia). - Hamps., Cat. Lep. Phal. 1, p. 207 , t. 7 , f. 19 (1898).

einctata Hamps., Ann. \& Mag. Nat. Hist. (7) 15, p. 428 $(1905)$.

cosmosomoides Rothsch., Nov. Zool. 18, p. 30 (1911).

erocata Herr.-Schäff., Aussereur. Schmett. 1, p. 73, f. 248 (1851) (Glaucopis). - Hamps., Cat. Lep. Phal. 1, p. 209 (1898).

crusmatica Dogn., Hét. nouv. Am. Sud 2, p. 5 (1911).

diaphana Sepp, Surin. Vlind. 2, p. 185, t. 81 (1848) (Glaucopis). - Hamps., Cat. Lep. Phal. 1, p. 209 (1898).

discifera Walk., List Lep. Ins. Br. Mus. 1, p. 178 (1854) (Glaucopis).

bura Buti., Illustr. Het. Br. Mus. 1, p. 33, t. 11, f. 12 (1877) (Laemocharis) (nec Herr.-Schäff.).

albifrons Möschl., Stett. Ent. Zeitg. 33, p. 344 (1872) (Chrysostola).

Biolog i e: Sepp, Surin. Vlind. 2, p. 185, t. 81 (1848). - Hamps., Cat. Lep. Phal. 1, p. 209 (1898). egregia Schaus, Ann. \& Mag. Nat. Hist. (8) 7, p. 174 (1911). eumonides Druce, Proc. Zool. Soc. Lond. p. 378 (1883) (Desmidocnemis). - Hamps., Cat. Lep. Phal. 1, p. 211 , t. 8 , f. 2 (1898).

Puinigra Kaye, The Ent. 44, p. 145 (1911).

fiavinigra Jones, Trans. Ent. Soc. Lond. p. 144 (1908).

flavipicta Schaus, Ann. \& Mag. Nat. Hist. (8) 9, p. 34 (1912).

geminata Schaus, Proc. U. S. Nat. MIus. 29, p. 185 (1905).

Imitata Druce, Biol. Centr.-Am., Het. 1, p. 62, t. 7, f. 23 (1884); 2, p. 347 (1897) (Dycladia). - Hamps., Cat. Lep. Phal. 1, p. 208 (1898).

inconsplcuata Kaye, The Ent. 44, p. 145 (1911).

Kingesi Rothsch., Nóv. Zool. 18, p. 29 (1911).

Peru

Ecuador,

Südost-

brasilien

Santa

Catharina

Venezucla

Surinam

Franz.-

Guayana,

Rio Janeiro

Frenz.-

Guayana

Surinam,

Pará

Costarica

Ecuador

São Paulo

Paraná

Costarica

Franz.-

Guayana

Guatemala, Honduras

Brit.Guayana

Trinidad, Venezuela, Surinam 
masa Druce, Ann. \& Mag. Nat. Hist. (6) 4, p. 83 (1889) (Laemocharis); Biol. Centr.-Am., Het. 2, p. 341, t. 71, f. 10 (1897). - Hamps., Cat. Lep. Phal. 1, p. 209 (1898).

metamelaenae (metamela) Dogn., Hét. nouv. Am. Sud 3, p. 3 (1911).

peralta Schaus, Ann. \& Mag. Nat. Hist. (8) 9, p. 34 (1912).

picta Walk., List Lep. Ins. Br. Mus. 1, p. 176 (1854) (Glaucopis). - Butl., Illustr. Het. Br. Mus. 1, p. 32, t. 11, f. 4 (1877). - Hamps., Cat. Lop. Phal. 1, p. 207 (1898).

postflaria Druce, Ann. \& Mag. Nat. Hist. (7) 1, p. 407 (1898). - Hamps., Cat. Lep. Phal. 1, p. 210 , t. 8, f. 4 (1898).

pyrgion Druce, Biol. Centr.-Am. Het. 1, p. 62, t. 7, f. 22 (1884) (Dycladia). - Hamps., Cat. Lep. Phal. 1, p. 208, f. 99 (1898).

rufescens Rothsch., Nov. Zool. 18, p. 29 (1911).

semiflava Rothsch., Nov. Zool. 18, p. 30 (1911).

socerrensis Dogn., Hét. nouv. Am. Sud 2, p. 4 (1911).

splendens Möschl., Stett. Ent. Zeitg. 33, p. 345 (1872) (Chrysostola). - Hamps., Cat. Lep. Phal. 1, p. 208, t. 7, f. 20 (1898).

triangulifera Feld., Reise Nov., Lep. 2, p. 9, t. 102, f. 34 (1869) (Sphenoptera). - Hamps., Cat. Lep. Phal. 1, p. 211 (1898).

vesparis Butl., Ann. \& Mag. Nat. Hist. (4) 12, p. 227 (1873) (Poecilosoma). - Hamps., Cat. Lep. Phal. 1, p. 210, f. 100 (1898).

30

\section{Mesothen Hamps.}

Hamps., Cat. Lep. Phal. 1, p. 212 (1898).

albifrons Schaus, Journ. New York Ent. Soc. 9, p. 41 (1901).

aurantiaca Dogn., Ann. Soc. Ent. Belg. 50, p. 178 (1906). caeruleicorpus Schaus, Proc. U. S. Nat. Mus. 29, p. 185 (1905).

eatheriua Schaus, Proc. Zool. Soc. Lond. p. 275 (1892) (Dycladia); Am. Lep. p. 5, t. 1, f. 12 (1892). - Hamps., Cat. Lep. Phal. 1, p. 214 (1898).

desperata Walk., List Lep. Ins. Br. Mus. 7, p. 1602 (1856) (Pseudomya). - Hamps., Cat. Lep. Phal. 1, p. 215 , t. 8 , f. 3 (1898).

doris Druce, Ann. \& Mag. Nat. Hist. (8) 8, p. 138 (1911). dorsimacula Rothsch., Nov. Zool. 18, p. 45 (1911). endoleuca Druce, Anu. \& Mag. Nat. Hist. (7) 15, p. 460 (1905).

epimetheus Sehaus, Proc. Zool. Soc. Lond. p. 275 (1892) (Dycladia); Am. Lop. p. 5, t. 1, f. 14 (1892). - Hamps., Cat. Lep. Phal. 1, p. 213 (1898).

erythraema (erythaema) Hamps., Cat. Lep. Phal. 1, p. 214 , t. 9, f. 2 (1898).

ethela Schaus, Ann. \& Mag. Nat. Hist. (8) 7, p. 175 (1911).

Ilavicostata Druce, Ann. \& Mag. Nat. Hist. (7) 18, p. 77 (1906).
Mexico,

Honduras

Südamerika

(ubi?)

Costarica

Pará

Franz.-

Guayana

Panama,

Franz. -

Guayana

Venezuela

Colombia

Surinam

Venezuela,

Amazonas, São Paulo

Peru,

Bolivia,

Colombia

(M.C.Vind.)

Colombia

Peru

Bolivia

Santa

Cutharina

Panama,

Amazonas,

Sāo Paulo,

Paraguay

Colombia

Costarica

Venezuela

Rio Janeiro

Colombia

Costarica

Peru 

llaviventris Druce, Biol. Centr.-Am., Het. 1, p. 62, t. 8,
f. 12 (1884) (Dycladia). - Hamps., Cat. Lep. Phal. 1, p. 214 (1898).

ignea Druce, Ann. \& Mag. Nat. Hist. (7) 1, p. 403 (1898). - Hamps., Cat. Lop. Phal. 1, p. 213, t. 8, f. 21 (1898).

montana Schaus, Ann. \& Mag. Nat. Hist. (8) 7, p. 175 (1911).

mysia Druce, Ann. \& Mag. Nat. Hist. (7) 18, p. 77 (1906).

nana(-um) Schaus, Proc. U. S. Nat. Mus. 29, p. 185 (1905).

nomir Druce, Ann. \& Mag. Nat. Hist. (7) 5, p. 507 (1906).

Oekendeni Druce, Ann. \& Mag. Nat. Hist. (7) 15, p. 460 (190б).

perllava Kayc, The Ent. 44, p. 145 (1911).

petosiris Druce, Proc. Zool. Soc. Lond. p. 376 (1883) (Loxophlebia). - Hamps., Cat. Lep. Phal. 1, p. 212 , t. 8 , f. 1 (1898).

pyrrha Schaus, Ent. Am. 5, p. 89 (1889) (Dycladia). Druce, Biol. Centr.-Am., Het. 2, p. 348, t. 71, f. 27 (1897). - Hamps., Cat. Lep. Phal. 1, p. 214, f. 101 (1898).

Rogenhoferi Schaus, Proc. Zool. Soc. Lond. p. 275 (1892) (Dycladia); Am. Lep. p. 5, t. 1, f. 13 (1892). - Hamps., Cat. Lep. Phal. 1, p. 212 (1898).

samina Druce, Ann. \& Mag. Nat. Hist. (6) 18, p. 30 (1896) (Homoeocera); Biol. Centr.-Am., Het. 2, p. 340, t. 71, f. 9 (1897). - Hamps., Cat. Lep. Phal. 1, p. 213 (1898).

temperata Schaus, Ann. \& Mag. Nat. Hist. (8) 7, p. 174 25 (1911).

Panama

Venezuela

Costarica

Peru

Surinam

Colombia

Peru

Sāo Paulo

Ecuador,

Bolivia

Mexico,

Franz.-

Guayana,

Colombia

Rio Janeiro

Panama

Costarica

\section{Chrostosoma Hübn.}

Hübn., Verz. bek. Schmett. p. 126 (1826). - Hamps., Cat. Lop. Phal. 1, p. 215 (1898).

Laemocharis Herr.-Schäff., Aussereur. Schmett. 1, p. 80 (1854). Heterodontia Feld., Reise Nov., Lep. Atlas p. 5 (1868).

eardinalis(-e) Schaus, Journ. New York Ent. Soc. 6, p. 139 (1898). - Hamps., Cat. Lep. Phal. 1, p. 217, t. 8, f. 8 (1898).

decisa Walk., List Lep. Ins. Br. Mus. 31, p. 81 (1864) (Pseudomya). - Hamps., Cat. Lep. Phal. 1, p. 215, f. 102 (1898).

echemus Cram., Pap. Exot. 4, p. 147, t. 367, f. B (1782) (Sphin $x$ ). - Hamps., Cat. Lep. Phal. 1, p. 216 (1898).

dolens Walk., List Lep. Ins. Br. Mus. 1, p. 148 (1854) (Glaucopis).

stulta Hcrr.-Schäff., Aussereur. Schmett. 1, p. 73, f. 258 (1854) (Glaucopis).

fumosa Schaus, Ann. \& Mag. Nat. Hist. (8) 9, p. 35 (1912). haematica Perty, Delect. Anim. Artic. p. 156, t. 31, f. 6 (1834) (Glancopis). - Hamps., Cat. Lep. Phal. 1, p. 216 (1898).

zantes Herr.-Schäff., Aussereur. Schmett, 1, p. 73, f. 240 (1854) (Glaucopis).
Colombia

Amazonas

Surinam, Pará, Rio Janeiro

Costarica

Ecuador, Brasilien (ubi?) 
fenestrina Butl., Jouin. Linn. Soc. Lond., Zool. 12, p. 383 (1876) (Laemocharis).

chalconitis Druce, Proc. Zool. Soc. Lond. p. 378, t. 39 , f. 10 (1883) (Dycladia)

maratha Druce, Ann. \& Mag. Nat. Hist. (7) 3, p. 229 (1899).

? mosea Dogn., Ann. Soc. Ent. Belg. 41, p. 24 (1897) (Laemocharis) (Inuius generis?).

patricia Schaus, Ann. \& Mag. Nat. Hist. (8) 9, p. 34 (1912).

pellueida Schaus, Proc. U. S. Nat. Kus. 29, p. 185 (1905).

Scinausi Rothsch., Nov. Zool. 18, p. 30 (1911).

scmirubra (-um) Hamps., Cat. Lep. Phal. 1, p. 217, t. 9, f. 1 (1898).

sitiona Schaus, Ann. \& Mag. Nat. Hist. (8) 7, p. 175 (1911).

tricolor Fold., Reiso Nov., Lep. 2, p. 5, t. 105, f. 25 (1868) (Heierodontia). - Hamps., Cat. Lep. Phal. 1, p. 217 , f. 103 (1898).

unxa Drucc, Ann. \& Mag. Nat. Hist. (6) 18, p. 31 (1896) (Ilipa); Biol. Centr.-Am., Het. 2, p. 346, t. 71, f. 24 (1897). - Hamps., Cat. Lep. Phal. 1, p. 216 (1898).

viridipuuctata(-um) Rothsch., Nov. Zool. 18, p. 30 (1911). 15

Ecuador

Ecuador

Costarica

Peru

Panama, Ecuador Sĩo Paulo

Costarica

Amazonas

Panama

Bolivia

\section{Nyridela Lucas.}

Lucas in Sagra, Hist. Cuba, Ins. p. 663 (1857). - Hamps., Cat. Lep. Phal. 1, p. 218 (1898).

Hochloptera Butl., Journ. Linn. Soc. Lond., Zool. 12, p. 386, t. 27 , f. $15(1876)$.

chalciope Hübn., Zutr. Exot. Schmett. 3, p. 20, f. 469, 470 (1827) (Isunthrene). - Hamps., Cat. Lcp. Plial. 1, p. 218 (1898).

acroxantha Perty, Delect. Anim. Art. p. 156, t. 31, f. 4 (1834) (Gluucopis).

Biologic: Dewitz, Nova Acta Acad. Lcop. Car. 44, p. 251, t. 1 , โ. 5 (1882).

xanthocera Walk., List Lep. Ins. Br. Mus. 7, p. 1603 (1856) (Gymnelia). - Drucc, Biol. Centr.-Am., Het. 1, p. 58 (1884); 2, p. 343 (1897). - Hamps., 2 Cat. Lep. Phal. 1, p. $21 \%$ f. 104 (1898).

Cuba, Colombia, Brasilien (ubi?)

Mexico, Guatemala, Costarica, Panama

\section{Leucotmemis Butl.}

Butl., Journ. Linn. Soc. Iond.: Zool. 12, p. 391, t. 27, f. 12 (1876). Hamps., Cat. Lep. Phal. 1, p. 219 (1898).

albiguita Schaus, Proc. U. S. Nat. Mus. 29, p. 185 (1905). bella Kaye, The Ent. 44, p. 144 (1911).

climacina Butl., Journ. Linn. Soc. Lond., Zool. 12, p. 394 (1876) (Dycladia). - Hamps., Cat. Lep. Plial. 1 , p. 223 , t. 8 , f. 22 (1898).

aiorsalis Walk., List Lep. Ins. Br. Mius. 1, p. 177 (1854) (Glancopis). - Butl., Illustr. Het. Br. Mus. 1, p. 33, t. 11, f. 6 (1877). - Hamps., Cat. Lep.

Surinam

Sño Pañlo

IEspiritı

Santo

Parḱ Phal. 1, p. 219 (1898). 
cmergens Walk., List Lcp. Ins. Br. Mus. 31, p. 92 (1864) (Eurota). - Hamps., Cat. Lep. Phal. 1, p. 223, t. 8 , f. 23 (1898).

endochrysa Dogn., Hét. nour. Am. Sud 3, p. 4 (1911).

Folderi Rothsch., Nov. Zool. 18, p. 30 (1911).

hemilenea Butl., Journ. Linn. Soc. Lond., Zool. 12, p. 393 (1876) (Dycladia). - Hamps., Cat. Lcp. Phal. 1, p. 221 , t. 8 , f. 7 (1898).

inspernta Walk., List Lep. Ins. Br. Mus. 7, p. 1606 (1856) (Poecilosoma). - Hamps., Cat. Lop. Phal. 1, p. 224, t. 8, f. 25 (1898).

interseeta Wralk., List Lep. Ins. Br. Mus. 31, p. 91 (1864) (Eurota). - Hamps,, Cat. Lep. Phal. 1, p. 222, t. 8 , f. 27 (1898).

latilines Walk., List Lep. Ins. Br. Mus. 1, p. 167 (1854) (Glaucopis ). - Butl., Illustr. Het. Br. Nus. 1, p. 32, t. 11 , f. 10 (1877). - Hamps., Cat. Lop. Phal. 1, p. 222 (1898).

margaritifera (margariphera) Butl,. Journ. Linn. Soc. Lond., Zool. 12, p. 393 (1876) (Dycladia). Hamps., Cat. Iep. Phal. 1, p. 220 , t. 8, f. 5 (1898).

nexa Herr.-Schäff., Anssereur. Schmett. 1, p. 73, f. 254 (1854) (Glancupis). - Druce, Biol. Centr.-Am. Het. 1, p. 44 (1884); 2, p. 335 (1896). - Hamps., Cat. Lep. Phal. 1, p. 224 (1898).

omole Druce, Proc. Zool. Soc. Lond. p. 377 (1883) (Cosmosoma). - Hamps., Cat. Lep. Phal. 1, p. 222, t. 8, f. 26 (1898).

oruatula Walk., List Lep. Ins. Br. Mus. 1, p. 163 (1854) (Glaucopis). - Hamps., Cat. Lep. Phal. 1, p. 224, t. 8 , f. 24 (1898).

pleursematr Hamps., Cat. Lep. Phal. 1, p. 221, t. 8, f. 6 (1898).

v. paranensis Schrottky, Iris 24, p. 150 (1910). Biologic: Schrottley, Iris 24, p. 150 (1910).

tenthredinoides (tonthredoides) Walk., List Lep. Ins. Br. Mus. 7, p. 1610 (1856) (Ilipa). - Hamps,, Cat. Lep. Phal. 1, p. 221 , f. 105 (1898).

thorneicn Schaus, Proc. U. S. Nat. Mus. 29, p. 186 (1905).

torrida Walk., List Lep. Ins. Br. Mus. 1, p. 161 (1854) (Glaucopis). - Hamps., Cat. Lep. Phal. 1, p. 223, t. 8 , f. 28 (1898).

varipes Walk., List Lep. Ins. Br. Mus. 1, p. 175 (1854) (Glaucopis). - Butl., Illustr. Het. Br. Mus. 1, p. 32, t. 11, f. 5 (1877). - Hamps., Cat. Lop. Phal. 1, p. 220 (1898).

albiventris Walk., List Lep. Ins. Br. Mus. 1, p. 176 (1854) (Glaucopis).

syrphiformis Herr.-Schäff., Ausscreur. Schmett. 20
Amazoras

Franz.-

Guayana

Amazonas

Peru

Pará

Surinam,

Amazonas

Brasilien

(ubi ?)

Pará

Mecxio,

Guatemala,

Honduras,

Costarica,

Colombia,

Amazonas,

Pará

Eenador

Pará

Santa

Catharina

Paraguay, Argentinicn

Amazonas

Franz.-

Pará

Guayana

Pará,

Rio

Janeiro 
Cosmosoma Hübn.

Hübu., Samml. Exot. Sehmett. 2 (1827). - Harris, Am. Journ. Se. 36, p. 317 (1839). - Butl., Journ. Linn. Soc. Lond., Zool. 12, p. 386, t. 27, f. 3 (1876). - Möschl., Verh. zool.-bot. Ges. Wien 27, p. 635 (1875). Hamps., Cat. Lep. Pual. 1, p. 225 (1898).

Lngaria Walk., List Lep. Ins. Br. Mus. 1, p. 164 (1854) (nce Dallas 1852).

Erruca Walk., List. Lep. Ins. Br. Mus. 1, p. 165 (1S54). - Butl., Journ. Linn. Soe. Lond., Zool. 12, p. 377, t. 27, f. 7 (1876).

llipa WValk., List. Lep. Ins. Br. Mus. 1, p. 166 (1854).

Aristoda ema Wallengr., Ofvers. Srenska Akad. Förh. 15, p. 136 (1858).

Glaucopis Burm., Descr. Phys. Rep. Argent. 5, p. 373 (1878) (nee Hiibn.).

Rezin Kirby, Cat. Het. 1, p. 149 (1892).

Biologie: Haase, Bibl. Zool. (2) 12, p. 76 (1893) (Mimicry).

aehemon Fabr., Spec. Ins. 2, p. 162 (1781) (Zygaena). - Hamps., Cat. Lep. Phal, 1, p. 247 (1898). - Klages, Proe. U. S. Nat. Mus. 29, p. 536 (1906).

tyrrhene Hübn., Zutr. Exot. Schmett. 3, p. 23, f. 483,484 (1827) (Euchromia).

voltumna Druco, Ann. \& Mag. Nat. Hist. (6) 20, p. 303 (1897).

ab. bolivarense (-is) Klages, Proc. U. S. Nat. Mus. 29, p. 536 (1906).

aehenoniles Dogn., Ann. Soe. Ent. Belg. 51, p. 223 (1907).

ada Herr.-Schäff., Aussereur. Schmett. 1, p. 73, f. 297 (1855) (Glaucopis). - Druce, Biol. Centr.-Am., Het. 2, p. 343 (1897).

aduotum(-a) Herr.-Sehäff., Aussereur. Schmett. 1, p. 73, f. 241 (1854) (Glaucopis). - Hamps., Cat. Lep. Phal. 1, p. 248 (1898).

ab.confine(-is) Herr.-Sehäff., Aussereur. Schmett. 1, p. 73, f. 255 (1854) (Glaucopis). - Druce, Biol. Centr.-Am., Het. 1, p. 59 (1884). - Hamps., Cat. Lep. Phal. 1, p. 248 (1898).

alvena Druce, Biol. Centr.-Am., Het. 1, p. 59, t. 7, f. 15 (1884); 2, p. 344 (1897). - Hamps., Cat. Lep. Phal. 1, p. 250 (1898).

analieinetum(-n) Rothseh., Ann. \& Mag. Nat. Hist. (8) 5, p. 509 (1910).

angustimargo Schaus, Ann. \& Mag. Nat. Hist. (8) 7, Costariea p. 177 (1911).

anıexum(-a) Herr.-Sehäff., Aussereur. Sehmett. 1, p. 73, f. 257 (1854) (Glaucopis). - Hamps., Cat. Lep. Phal. 1, p. 249 (1898).

anoxantlium(-a) Druce, Ann. \& Mag. Nat. Hist. (7) 15, Venezuela p. 460 (1905).

apenninum(-a) Druee, Ann. \& Mag. Nat. Hist. (7) 15, Peru p. $460(1905)$.

Jamaica,

Haiti,

Venezuela,

Pará

Venezuela

Peru

Panama, Amazonas, (M. C.

Vind.), Rio

Janeiro

Mexieo (MI.

C. Vind.), Guatemala, Pernam-

buco,

Bahia,

Espiritu

Santo

Mexico,

Guatemala

Peru

Rio Janeiro, São Paulo 
auge L., Syst. Nat. ed. 12, p. 807 (I767) (Sphinx). Druec, Biol. Centr.-Am. Het. 1, p. 60 (1884). - Hamps., Cat. Lep. Phal. I, p. 227 (1898). - Sclurottky, Iris 22, p. 124 (1909).

omphale Hübn., Samml. Exot. Schmett. 2 (1827). - Burm., Deser. Phys. Rev. Argent. 5, p. 374 (1878).

melitta Mösclıl., Verl. zool.-bot. Ges. Wien 27, p. 635 , t. 8 , f. 3 (1877).

Biologie: Dewitz, Nova Acta Acad. Leop.-Carol.44, p. 251, t. 1, f. 4 (1882). - Gundlach, Ent. Cub. p. 239 (1886).

Baroni Rothsch., Nov. Zool. 18, p. 32 (1911).

Batesi(-ii) Butl., Journ. Linn. Soc. Lond., Zool. 12, p. 394 (1876) (Dycladia). - Hamps., Cat. Lcp. Phal. 1, p. 239, t. 9, f. 7 (1898).

beatrix Druce, Biol. Centr.-Ain., Het. 1, p. 51, t. 6, f. 25 (1884); 2, p. 340 (1897) (Homoeocera). - Hamps., Cat. Lep. Phal. 1, p. 233 (I898).

scintillan Druce, Biol. Centr.-Am., Het. 1, p. 51 (1884) (llomoeocera) (nce Herr.-Schäff.).

beatum(-n) Butl., Journ. Linn. Soc. Jond., Zool. 12, p. 376 (1876) (Homoeocera). - Druce, Biol. Centr.-Am.. Het. 1, p. 52 (1884). - Hamps., Cat. Lep. Phal. 1, p. 234, t. 8, f. 15 (1898).

biseriatum Schaus, Journ. Now York Ent. Soc. 6, p. 138 (1898). - Hamps., Cat. Lep. Phal. 1, p. 251, t. 9, f. 16 (1898).

bogotense(-is) Feld., Reise Nov., Lep. Atlas p. 4, t. 102, f. 32 (1869) Euchromia). - Hamps., Cat. Lep. Phal. 1, p. 254 (1898).

Bolivari Schaus, Journ. Now York Ent. Soc. 6, p. 138 (1898). - Hamps., Cat. Lep. Phal. 1, p. 238, t. 9 , f. 3 (IS98).

braconoides Walk., List Lep. Ins. Br. Mus. 1, p. 166 (1854) (Glaucopis). - Butl., Illustr. Het. Br. Ius. 1, p. 32, t. 11, f. 15 (1877). - Druce, Biol. Centr.-Am,, Het, 2, p, 346 (1897). — Hamps., Cat. Lep. Phal. 1, p. 253 (1898).

brasiliense Rothsch., Nov. Zool. 18, p. 36 (1911).

Bricenoi Rotlusch., Nov. Zool. 18, p. 32 (1911).

Brinkleyi Rothsch., Nov. Zool. 18, p. 31 (1911).

? bromus Cram., Pap. Exot. 1, p. 56, t. 35, f. G (1775) (Sphinx). - Hamps., Cat. Lep. Phal. 1, p. 239 (1898) (huius generis?).

Bueliwaldi Rothsch., Ann. \& Mag. Nat. Hist. (8) 5, p. 509 (1910).

eaecum Hamps., Cat. Lep. Phal. 1, p. 246 (1898). impar Druce, Biol. Centr.-Am., Het. 1, p. 60 (pr. p.) (1884); 2, p. 345, t. 71, f. 22 (1897) (nce Walk.).

earabayanum Rothsch., Nov. Zool. 18, p. 32 (1911). cardinale Hamps., Cat. Lep. Phal. 1, p. 230, t. 8, f. 16 (1898).

- eentralc(-is) Walk., List Lcp. Ins. Br. Mus. 1, p. 171 (1854) (Glaucopis). - Butl., Jllustr. Het. Br.
Cuba,

Jamaica,

St. Thomas

Mexico,

Guatemala,

Costarica,

Panama,

Surinau,

Amazonas

(M.C.Vind.),

São Paulo,

Paraguay,

Uruguay,

Argentinicn

Ecuador

Pará,

São Paulo

Costarica,

Panama

Panama,

Colombia,

Brasilien

(ubi?)

Ecuador

Colombia

Ecuador

Mexico

Honduras

Santa

Catharina

Venezuela

Colombia

Surinam

Ecuador

Mexico, Guatcmaln, Costarica Panama

Peru

São Paulo

Panama, Amazonas 
Mus. 1, p. 31, t. 11, f. 9 (1ST7). - Hamps., Cat. Lep. Phal. 1, p. 243 (1898).

bura Herr.-Schäff., Aussereur. Schmett. 1, p. 73, f. 239 (1854) (Laemocharis). - Druce, Biol. Centr.-Am., Het. 2, p. 343 (1879).

cermena Dugn., Ann. Soc. Ent. Belg. 43, p. 250 (1899). chiriquense(-is) Rothsch., Ann. \& Mag. Nat. Hist. (8) 5, p. 508 (1910).

cinctuni(-a) Schaus, Proe. Zool. Soc. Lond. p. 22 ã (1894) (Homoeocera). - Hamps., Cat. Lep. Phal. 1, p. 232 , t. 8 , f. 12 (1898).

cinctutumi(-n) Schaus, Ann. \& Mag. Nat. Hist. (8) 7 , 1. 176 (1911)

citrinum Rothsch., Nov. Zool. 18, p. 34 (1911).

colona Schaus, Ann. \& Mag. Nat. Hist. (8) 7, p. 177 (1911).

consolatumi(-a) Walk., list Jep. Ins. Br. Mus. 7, p. 1601 (1856) (Pseudomya). - Hamps., Cat. Lep. Phal. 1, p. 248 , t. 9, f. 26 (1898).

contractumı(-a) Walk., List Lep. Ins. Br. Mus. 7, p. 1609 (1856) (Laemocharis). - Hamps., Cat. Lep. Phal. 1, 1). 237, t. 8, f. 20 (1898).

corvicum(-a) Dogiı., Hét. nouv. Am. Sud 1, p. 4 (1910). crathidinum Rothsch., Nov. Zool. 18, p. 34 (1911).

crucutum(-a) Perty, Delect. Anim. Artie. p. 157, t. 31, f. 8 (1834) (Glaucopis). - Hamps., Cat. Lop. Phal. 1, p. 246 (189S).

demantria Druce, Ann. \& Mag. Nat. Hist. (6) 15, p. 44 (1895). - Hamps., Cat. Lep. Phal. 1, p. 226, t. 8, f. 9 (1598).

determinatum(-a) Butl., Journ. Tinn. Soc. Lond., Zool. 12, p. 391 (1876) (Ilipa). - Hamps, Cat. Lep. Phal. 1, p. 240, t. 9, f. 13 (1898).

Deyrollei(-ii) Walk., List Lep. Ins. Br. Mus. I, p. 165 (1854) (Glancopis). - Butl., Mllustr. Het. Br. Mus. 1, 1. 28, t. 12, f. 6 (1877). - Hamps., Cat. Lep. Phal. 1, p. 238 (1898).

Iorsimaculum(-a) Sehaus, Journ. Netw York Ent. Soe. 6, p. 138 (1898). - Hamps., Cat. Lep. Phal. 1, p. 252 , t. 9 , f. 15 (1898).

dubium Rothsch., Nor. Zool. 18, p. 34 (1911).

durea Schaus, Journ. Netw York Ent. Soc. 4, p. 132 (1896). - Hamps., Cat. Lep. Phal. 1, p. 241, t. 9 , f. $23(1898)$.

? nigripes Hoyl., C. R. Soe. Ent. Bolg. 34, p. XIV (1890) (Laemocharis) (nomen prius, sedineertum).

elegans Butl., Journ. Linn. Soc. Lond., Zool. 12, p. 386 (1876). - Hamps., Cat. Lep. Plial. I, p. 241, t. 9, f. 12 (1898).

cutella Druce, Ann. \& Mlag. Nat. Hist. (7) 3, p. 229 (1899). crythrarchum(-0s) Walk., List Lep. Ins. Br. Mus. 1, p. 164 (1851) (Glaucopis). - Hamps., Cat. Lep. Phal. 1, p. 230 (1898).

vulnerata Hcrr.-Schälf., Aussereur. Schmett. I, p. 73, f. 238 (1854) (Glaucopis). - Butl., Mlustr. Het. Br. Mus. 1, p. 36, t. 12, f. 2 (1877).

ethodaea Druce, Ann. \& Mag. Kat. Hist. (6) 4, p. 84 (1889); Biol. Centr.-An., Het. 2, p. 344 , t. 71, f. 20 (1897). - Hamps., Cat. Lep. Phal. 1, p. 249 (1898).
(1I. C.

Vind.),

RioJaneiro,

São Paulo,

Paraguay

Ecuador

Panama

Venczuela

Costariea

Patria?

Costariea

Brasilien

(ubi?)

São Paulo,

Paraguay

Bolivia

Patria?

Amazonas

Dominiea

Colombia

Rio Grande do Sul

Ecuador

Jamaiea

São Paulo

lispiritu

Santo

Ecuador

Venezuela,

Rio Janeiro

Mexico 
enmelis Druce, Proc. Zool. Soc. Lond. p. 376 (1883) (Loxophlebia). - Hamps., Cat. Lep. Phal. 1, p. 249 , t. 9 , f. 28 (1898).

? evadnes Cram. Pap. Exot. 4, p. 129, t. 357, f. A (1728) (Sphinx). - Hamps., Cat. Lep. Phal. 1, p. 251 (1898) (huius gencris?).

Biologic: Stoll., Suppl. Cram. p. 68, t. 15, f. 1-3 (1790).

exomelan Zerny nom. nov.

Mathani Rotlisch., Nov. Zool. 18, p. 31 (1911) (nec Rothsch. 1910).

fenestratum(-a) Drury, Ill. Exot. Ent. 1, p. 54, t. 25, f. 3 (1770) (Glancopis). - Stoll, Suppl. Cram. p. 140 , t. 30 , f. 5 (1790). - Hamps., Cat. Lep. Phal. 1, p. 236 (1898).

steropes Göze, Ent. Beitr. 3 (2), p. 215 (1780) (Sphinx).

rubripeda Lncas in Sagra, Hist. Cuba, Ins. p. 658 (1857) (Glaucopis).

festivum(-a) Walk., List Lep. Ins. Br. Mus. 1, p. 171 (1854). - Druce, Biol. Centr.-Am., Het. 2, p. 345 (1897). - Hamps., Cat. Lep. Phal. 1, p. 243 (1898).

aleus Scliaus, Ent. Am. 5, p. 89 (1889). - Druce, Biol. Centr.-Am. Het. 2, p. 344, t. 71, f. 16 (1897)

Biologie: Druce, Biol. Centr.-Am., Het. 2, p. 344 (1897).

flaricostale Rotliscli., Nov. Zool. 18, p. 33 (1911).

flavita Schaus, Journ. New York Ent. Soe. 9, p. 41 (1901).

flavitarse(-Is) Walk., List Lep. Ins. Br. Mus. 1, p. 158 (1854) (Glaucopis). - Hamps., Cat. Lep. Phal. 1, p. 233 (18.98).

scintillans Herr.-Schäff., Aussereur. Sehmett. 1, p. 73, f. 244 (1854) (Glaucopis). - Butl., Illustr. Hot. Br. Mus. 1, p. 26, t. 12, f. 4 (1877).

flarothoracides Zerny nom. nov.

intensa. Rothsch., Ann. \& Mag. Nat. Hist. (8) 5, p. 507 (1910) (nec Walk. 1854).

flavothorax Rothsch., Ann. \& Mag. Nat. Hist. (8) 5, p. $507(1910)$.

galaten (galathea) Sehaus, Ann. \& Mag. Nat. Hist. (8) 9 , p. 35 (1912).

galhanum(-a) Schaus, Ann. \& Mag. Nat. Hist. (8) 9 , p. 35 (1912).

Garleppi Rothsch., Nor. Zool. 18, p. 31 (1911).

gaza Schaus, Proc. Zool. Soc. Lond. p. 274 (1892) (Isanthrene); $\mathrm{Am}$. Lep. j. 3, t. 1, f. 9 (1896). Hamps., Cat. Lep. Phal. 1, p. 232 (1898).

genmutumı(-a) Butl., Journ. Iinn. Soc. Lond., Zool. 12 , p. 385 (1876) (Pheia); Illustr. Het. Br. Mus. 1, p. 30, t. 17, f. 5 (1877). - Druee, Biol. Centr.-Am. Het. 2, p. 343 (1897). - Hamps., Cat. Lep. Phal. 1, p. 237 (1898). - Klages, Proc. U. S. Nat. Mus. 29, p. 535 (1906).

ab. xanthocerum(-a) Hamps., Cat. Lep. Phal. 1, p. 236 , t. 9 , f. 5 (1898). - Klages, Proc. U. S. Nat. Mus, 29, p. 535 (1906).
Ecuador,

Bolivia,

São Paulo

Surinam

Colombia

Jamaica,

Cuba

Mexico,

Guatemala, Honduras,

Costarica

Peru

Colombia

Colombia, Venezuela, Brasilicn (ubi?)

Colombia, Ecuador

Patria?

Costarica

"

Bolivia

Peru

Panama,

Colombia,

Venezuela

Amazonas, Venezuela 
grueula Dogn., Hét. nouv. Am. Sud 2, p. 5 (1911).

guapila Sehaus, Ann. \& Mag. Nat. Hist. (8) 7, p. 176 (1911).

Irampsoni Klages, Proc. U. S. Nat. Mus. 29, p. 534 (1906).

hang: Horr.-Sehäff., Aussereur. Sehmett. I, p. 73, f. 246 (1854) (Glaucopis). - Hamps., Cat. Lep. Phal. 1, p. 231 (1898).

arauna Wallengr., Wien. Ent. Monatssehr. 4, p. II (1860) (Aristodaema).

harpalyce Schaus, Proc. Zool. Soe. Lond. p. 275 (1892); Am. Lep. p. 4, t. 1, f. 11 (1896). - Hamps., Cat. Lep. Plial. 1, p. 253 (1898).

hector Stgr., Verl. zool.-bot. Ges. Wien 25, p. 120 (1875) (Glancopis). - Diuce, Biol. Centr.-Am., Het. 1, p. 60, t. 7, f. 17 (1884). - Hamps., Cat. Lep. Phal. 1, p. 229 (1898).

hereyna Druce, Biol. Centr.-Am., Het. 1, p. 55, t. 7, f. 6 (1884) (Laemocharis). - Hamps,, Cat. Lep. Phal. 1, p. 253 (1898).

hypochilus (hypocheihs) Hamps., Trans. Eut. Soe. Lond. p. 242 (1898); Cat. Lep. Phal. I, p. 247, t. 9, f. 27 (1898).

lelnenmonoides Rothseh., Nov. Zool. 18, p. 34 (1911).

ignidorsium(-a) Hamps., Cat. Lep. Phal. 1, p. 227, t. 17, f. 17 (1898).

Biologie: Schrottky, Iris 24, p. 150 (1910).

impar Walk., List Lep. Ins. Br. Mus. 1, p. 169 (1854) (Glaucopis). - Druee, Biol. Centr.-Am., Het. 1, p. 60 (pr. p.), t. 7, f. 20 (1884). - Hamps., Cat. Lep. Phal. I, p. 230 (1898).

vernana Diuee, Biol. Centr.-Am., Het. 2, p. 346 (1897).

impudicum(-a) Selıaus, Ann. \& Mag. Nat. Hist. (8) 7 , p. 177 (1911).

intensum(-a) Walk., List Lep. Ins. Br. Mus. I, p. 159 (1854) (Clanecopis). - Butl., Hlustr. Het. Br. Mus. 1, p. 30, t. 12, f. 11 (1877). - Hamps., Cat. Lep. Phal. 1, p. 255 (1898).

? juanita Neum.-Can., Ent. 26, p. 335 (1894). - Hamps., Cat. Lep. Phal. I, p. 252 (1898) (huius generis?).

Klagesi Rothseh., Ann. \& Mag. Nat. Hist. (8) 5, p. 509 (1910).

ladan Druee, Ann. \& Mag. Nat. Hist. (6) 18, p. 31 (1896) (Dycladia): Biol. Centr.-Am., Het. 2, p. 348, t. 72 , f. 2 (1887). - Hamps., Cat. J.cp. Phal. 1, p. 255 (1898).

intensa Druce, Biol. Centr.-Am., Het. 1, p. 57, t. 7, f. 16 (1884) (Pheia) (nec Walk.).

Lemoulti Rothseh., Nov. Zool. 18, p. 156 (1911).
Colombia

Costarica

Venezuela

Amazonas

(AI. C.Vind.),

Rio Janeưo,

São Paulo,

Lspiritu

Santo,

Paraná

(M. C. Vind.),

Paraguay,

Argentinien

Rio Janeiro

Panama

Mexico, Niearagua

St. Vincent

Bolivia

Minas Geraes,

Santa

Cathariua

(M.C.Vind.),

Paraguay

Mexieo,

Guatemala,

Panama

Costarica

Honduras

Cuba

Trinidad

Panama

Franz. -

Guayana 
leuconotum(-on) Hamps., Cat. Lep. Phal. 1, p. 231, t. 8 , f. $11(1898)$.

lueens Dogn., Ann. Soc. Ent. Belg. 46, p. 226 (1902).

lycopolis Druce, Proc. Zool. Soc. Lond., p. 375, t. 39, f. 7 (1883) (Erruca). - Hamps., Cat. Lep. Phal. 1, p. 231 (1898).

Mathani Rothsch., Ann. \& Mag. Nat. Hist. (8) 5, p. 507 (1910)

molanoperum(-n) Hamps., Cat. Lep. Plial. 1, p. 240, t. 9, f. 6 (1898).

melanothoracium (melathoracia) Kayc, Trans. Ent. Soc. T.ond. p. 115 , t. 5, f. 10 (1901).

meridense Rothsch., Nov. Zool. 18, p. 36 (1911).

metalleseens Mén., Cat. Lép. Pétursb. 2, p. 138, t. 14, f. 1 (1857) (Laemocharis). - Hamps., Cat. Lep. Phal. 1, p. 239 (1898).

pheres var. Walk., List Lep. Ins. Br. Mus. 1, p. 162 (1854) (Glaucopis).

chalcosticta Butl., Journ. Linn. Soc. Lond. Zool. 12, p. 387 (1876).

metallieum Rothsch., Nov. Zool. 18, p. 33 (1911).

nyyodora Dyar, Journ. New York Ent. Soc. 15, p. 226 (1907).

auge Neum. \& Dyar, Journ. New York Ent. Soc. 1, p. 101 (1893). - Holl., Moth Book p. 98, t. 13, f. 1 (1903) (nec L.).

om phale Harr., Am. Journ. Sc. 36, p. 317 (1839). - Clem., Proc. Acad. Philad. p. 544 (1860). - Stretch, Zyg. \& Bomb. N. Am. p. 153, t. 7, f. 5 (1872). - Smith, Ent. Am. 1, p. 181 (1885) (nec Hübn.).

Biologie: Smith, Ent. Am. 1, p. 186 (1886). - Dyar, Psyche 7, p. 414 (1896); Proc. Boston Nat. Hist. Soc. 27, p. 139 (1896). - Hamps., Cat. Lep. Phal. 1, p. 228 (1898) (auge).

nelea Mlöschl., Verh. zool.-bot. Ges. Wien 27, p. 635, t. 8, f. 4 (1877). - Hamps., Cat. Lep. Phal. 1, p. 251 (1898).

nigrieorne(-is) Fabr., Mant. Ins. 2, p. 104 (1787) (Zygaena). - Hamps., Cat. Lep. Phal. 1, p. 234, t. 8 , f. 17 (1898).

Stretchii Butl., Journ. Linn. Soc. Lond., Zool. 12, p. 375 (1876) (Homoeocera).

nobile(-is) Schaus, Ann. \& Mag. Nat. Hist. (8) 7, p. 175 (1911).

notostictum(-a) Dogn., Ann. Soc. Ent. Belg. 53, p. 214 (1909).

Ockendeni Rothsch., Ann. \& Mag. Nat. Hist. (8) 5, p. 009 (1910).

oratlua Dince, Proc. Zool. Soc. Lond. p. 281 (1893) (Autochloris). - Hamps., Cat. Lep. Phal. 1, p. 241, f. 108 (1898).

orathidium(-a) Druce, Ann. \& Mag. Nat. Hist. (7) 1, p. 405 (1898). - Hamps., Cat. Lep. Phal. 1, p. 242 , t. 9 , f. 25 (1898).

Colomuia,

Amazonas

(M.C.Vind.)

Colombia,

Peru

(Ml.C.Vind.)

Ecuador

Colombia

Bolivia

Trinidad

Venezucla

Mexico, Panama

(MI. C.

Vind.),

Amazonas,

Pará

Colombia, Amazonas Florida

Surinam

Colombia

Costarica

Colombia

Peru

Brit.-

Guayana

Nicaragua 
oroyanum Rothsch., Nov. Zool. 18, p. 31 (1911).

Paranbac Rothsch., Nov. Zool. 18, p. 35 (1911).

pellucidum(-a) Lathy, Tho Ent. 32, p. 117 (1899).

Peru

Ecuador

Brasilien

(ubi ?)

perfenestratum Dyar,.Journ. New York Ent. Soc. 7,

Patria? p. 175 (1899).

pheres Cram., Pap. Fxot. 4, p. 183, t. 382 , f. C (1782) (Sphinx). - Hamps., Cat. Lep. Phal. 1, p. 239 (1898).

Biologic: Stoll, Suppl. Cram. p. 62, t. 13, f. 5 (1790).

phoenicophorum(-a) Dogn., Ann. Soc. Ent. Belg. 53, p. 214 (1909).

plagiatum(-a) Rothsch., Ann. \& Mag. Nat. Hist. (8) 5, p. 509 (1910)

plebceula Dogn., Ann. Soc. Ent. Belg. 46, p. 226 (1902).

plutonium (plutona) Schaus., Proc. Zool. Soc. Lond. p. 226 (1894). - Hamps., Cat. Lep. Phal. 1, p. 240 , t. 9 , f. 8 (1898).

protum(-us) Druce, Ann. \& Mag. Nat. Hist. (6) 13, p. 171 (1894); Biol. Centr.-Am., Het. 2, p. $3 \pm 4$, t. 71, f. 19 (1897). - Hamps., Cat. Lep. Phal. 1, p. 242 , f. 109 (1898).

psendothia Zerny nom. nov.

thia Schaus, Ann. \& Mag. Nat. Hist. (8) 9, p. 35 (1912) (nec Druce 1899).

pndicuu(-a) Druec, Ann. \& Nag. Nat. Hist. (6) 13, p. 353 (1894); Biol. Centr.-Am., Het. 2, p. 345, t. 71, f. 21 (1897). - Hamps., Cat. Lcp. Phal. 1, p. 251 , t. 9 , f. 14 (1898).

pytua Drucc, Ann. \& Mag. Nat. Hist. (7) 18, p. 78 (1906).

?quiuqucpunctatum(-n) Heyl., C. R. Soc. Ent. Belg. 34, p. XIII (1890) (Laemocharis). - Hamps., Cat. Lep. Phal. 1, p. 234 (1898) (huius generis?).

regium(-a) Schaus, Proc. Zool. Soc. Lond. p. 227 (1894) (Marissa). - Hamps., Cat. Lcp. Phal. 1, p. 254, t. 9, f. 10 (1598).

nicippe Druce, Ann. \& Mag. Nat. Hist. (6) 20 , p. 303 (1897) (Eunomia).

remotum(-a) Walk., List Lep. Ins. Br. Nus. 1, p. 170 (1854) (Claucopis). - Hamps., Cat. Lep. Plal. 1, p. 248 , t. 9, f. 22 (1898).

? riea Dogn., Ann. Soc. Ent. Belg. 41, p. 24 (1897) (Pheia) (huius generis?).

Rosenbergi Rothseh., Ann. \& Mag. Nat. Hist. (8) 5, p. 508 (1910).

rubrobasale(-is) Lathy, Tho Fint. 32 , p. 116 (1899).

rubriguttum(-4) Skinner, Ent. News 17, p. 96 (1906).

rubrlscapuluu(-ac) Kayc, Trans. Ent. Soc. Lond. p. 116, t. 5 , f. $9(1901)$.

rubritase(-is) Schaus, Ann. \& Mag. Nat. Hist. (8) 7 , p. 176 (1911).

SaIvini Butl., Journ. Linn. Soc. Lond., Zool. 12, p. 376 (1876) (Homoeocera); Illustr. Het. Br. Mus. 1, p. 27, t. 17, f. 4 (1877). - Druce, Biol. Centr.-Am., Het. 1, p. 52 (18S4). - Hamps., Cat. Lep. Phal. 1, p. 233 (1898).

Surinam,

Rio Janeiro

Franz.-

Guayana

Pcru

Colombia

Paraná

Mexico

Costariea

Mexico,

Costarica,

Panama

Trinidad

São Paulo

Venczucla,

Pcru

Venezuela

Ecuador

Ecuador,

Colombia

Ecuador

Arizona

'rinidad

Costariea

Panama 
sarou Druce, Biol. Centr.-Am., Het. 1, p. 59; t. 7, f. 14 (1884). - Hamps., Cat. Lep. Phal. 1, p. 238 (1898).

meres Druce, Ann. \& Mag. Nat. Hist. (6) 18, p. 30 (1896): Biol. Centr.-Am., Het. 2, p. 346 , t. 71, f. 23 (1897).

seitum(-n) Walk., List Lep. Ins. Br. Mus. 7, p. 1611 (1856) (Ilipa). - Hamps., Cat. Lep. Phal. 1, p. 250 , t. 9 , f. 9 (1898).

hilaris Walk., List Lep. Ins. Br. Mus. 31, p. 88 (1864) (Poecilosoma).

sectinotum(-a) Hamps., Cat. Lep. Phal. 1, p. 228, t. 8, f. $10(1898)$.

auge Druce, Biol. Centr.-Am., Het. 1, p. 60 (1884) (pr. p.)

semfiulvum(-n) Druce, Biol. Centr.-Am., Het. 1, p. 62, t. 8, f. 11 (1884); 2, p. 348 (1897) (Dycladia). - Hamps., Cat. Lcp. Phal. 1, p. 229 (1898).

sephela Druce, Proe. Zool. Soc. Lond. p. 375 (1883) (Erruca). - Hamps., Cat. Lep. Phal. 1, p. 235, t. 8 , f. 18 (1898).

? soruphinum(-n) Herr.-Schäff., Ausscreur. Schmett. 1, p. 74, f. 429 (1854) (Glancopis). - Hamps., Cat. Lep. Phal. 1, p. 236 (1898) (huius gencris?). sicula Dyar, Journ. Now York Ent. Soc. 7, p. 175 (1899). simillimum Rothsch., Nor. Zool. 18, p. 33 (1911). Stcinbachi Rothsch., Nor. Zool. 18, p. 33 (1911). stilbostictum(-a) Butl., Journ. Linn. Soc. Lond., Zool. 12, p. 391 (1876) (Ilipa). - Druec, Biol, Centr.-Am., Het. 1, p. 61 (1881). - Hamps., Cat. Lep. Phal. 1, p. 253, t. 9, f. 11 (1898).

stryua Druce, Biol. Centr.-Am., Het. 1, p. 55, t. 7, f. 5 (1884); 2, p. 341 (1897) (Lacmocharis). - Hamps., Cat. Lep. Phal. 1, p. 255 (1898).

Stuarti Rothsch., Nov. Zool. 18, p. 31 (1911).

subflammum(-a) Walk., List Lep. Ins. Br. Mus. 1 , p. 159 (1854) (Glancopis). - Hamps., Cat. Lep. Phal. 1, p. 227, f. 106 (1898).

panopes Herr.-Schäff., Aussereur. Schmett. 1, p. 73, f. 243 (1854) (Glaucopis); Butl., Illustr. Het. Br. Mius. 1, p. 31, t. 12, f. 9 (1877). Möschl., Verh. zool.-bot. Ges. Wien 27, p. 635 (1877).

v. lucia Schaus, Journ. Netw York Ent. Soc. 4, p. 132 (1896). - Hamps., Cat. Lep. Phal. 1, p. 227 (1898).

tarapotense(-is) Drucc, Ann. \& Mag. Nat. Hist. (6) 20, p. 302 (1597) (Homoeocera). - Hamps., Cat. Lep. Phal. 1, p. 232, t. 8, f. 14 (1898).

telephus Walk., List Lep. Ins. Br. Mus. 1, p. 170 (1854) (Glaucopis). - Herr.-Schäff., Ausscreur. Schmett. 1, p. 73, f. 242 (1854). - But1., Journ. Linn. Soc. Lond., Zool. 12, p. 388 (1876). - Burm., Descr. Phys. Rep. Argent. 5, p. 374 (1878). Hamps., Cat. Lcp. Phal. 1, p. 247 (1898).

coccine um Butl., Journ. Linn. Soc. Lond., Zool. 12, p. 388 (1876).
Panama

Amazonas

Mexico, Guatemala

Mexico,

Panama

Ecuador

Pará

Venezuela

Amazonas

Bolivia

Panama,

Colombia,

Ecuador

Mexico, Nicaragua

Amazonas

Surinam,

Venezuela, Amazonas (M.C.Vind.)

Santa Lucia

Peru,

Brasilicn

(ubi?, M. C. Vind.)

Colombia, Venezuela, Amazonas (II. C. Vind.), Espiritu Santo, Argentimien 
pyrrhostethus Butl., Journ. Linn. Soo. Lond., Zool. 12, p. 388 (1876).

tengyra Walk., List Lep. Ins. Br. Hus. 1, p. 167 (1854) (Glaucopis). - Hamps., Cat. Lep. Phal. 1, p. 252 (1898).

fulviventris Mén., Cat. Lóp. Pétersb. 2, p. 14l, t. 14, f. 5 (1857) (Laemocharis).

- teuthras Walk., List Lep. Ins. Br. Mus. 1, p. 168 (1854) (Glaucopis). - Butl., Illustr. Het. I3r. Mus. 1, p. 31, t. 13, f. 5 (1877). - Druce, Biol. Centr.-Am., Het. 1, p. 60 (1884). - Hamps., Cat. Lep. Phal. 1, p. 245 , f. 110 (1898). - Klages, Proc. U. S. Nat. Mus. 29, p. 536 (1906).

v. cingulatum Butl., Journ. Linn. Soc. Lond., Zool. 12, p. 389 (1876). - Dituce, Biol. Centr.-Am., Het. 1, 1. 60 (1881); 2, p. 345 (1S97). - Hamps., Cat. Lep. Phal. 1, p. 246 (1898).

v. erubescens Butl., Journ. Linn. Soc. Lond., Zool. 12, p. 389 (1876). - Hamps., Cat. Lcp. Phal. 1, p. 246 (1898).

v. lignicolor Rrutlisch., Nov. Zool. 18, p. 35 (1911). v. nigrescens Rothseh., Nov. Zool. 18, 1) 35 (1911). v. restrictum Butl., Journ. Linn. Soc. Lond., Zool. 12, p. 389 (1876). - Hamps., Cat. Lep. Phal. 1, p. 245 (1898).

thin Druec, Ann. \& Mag. Nat. Hist. (7) 3, p. 229 (1899). thoracieum Schaus, Proc. U. S. Nat. Mus. 29, p. 186 (1905).

tigris Schaus, Proc. Zool. Soc. I.ond. p. 226 (1894). Hamps., Cat. Lop. Phal. 1, p. 237, f. 107 (1898). venatum Rothsch., Nov. Zool. 18, p. 34 (1911).

resplarium(-a) Perty, Delect. Anim. Artic. p. 157, t. 31, f. 9 (1834) (Glancopis). - Hamps., Cat. Lep. Phal. 1, p. 250 (1898).

odyneroides Walk., List Lep. Ins. Br. Mus. 7, p. 1604 (1856) (Isanthrene).

villia Druce, Ann. \& Mag. Nat. Hist. (7) 18, p. 78 (1906). viridieingulatuu Rothsch., Nov. Zool. 18, p. 32 (1911).

Watsoui Rothsch., Ann. \& Mag. Nat. Hist. (8), 5 p. 508 (1910).

xanthistis Hamps., Cat. Lep. Phal. 1, p. 231, t. 9, f. 4 (1898).

xuntlomelan Zcrny nom. nov.

Rosenbergi. Rotliseli., Nov. Zool. 18, p. 35 (1911) (nec. Rothsch. 1910).

xunthostietum Hamps., Cat. Lep. Phal. 1, p. 240, t. 9, f. 21 (1898).

metallescens Druce, Biol. Ccntr..Am., Het. 1 , p. 58 (1884); 2, p. 343 (1897) (nec. Mlén.).

zelosum(-a) Dogn., Ann. Soc. Ent. Belg. 43, p. 251 (1899).

Zureheri Druec, Ann. \& Mag. Nat. Hist. (6) 13, p. 353 (1894); Biol. Centr. Am., Het. 2, p. 345, t. 71, f. 18 (1897). - Hamps., Cat. Lep. Phal. 1, p. 229, 150 t. 8 , f. 19 (1898).

Pará

Mexico,

Panama,

Culombia,

Venezuela,

Amazonas

(M.C.Vind.),

Bahia

(M.C.Vind.),

Paraguay,

Argentinien

Mexieo,

Guatemala

Costarica,

Panama

São Paulo

Ecuador

Colombia

Pará

Ecuador

Franz.-

Guayana

Venezucla

Peru

Amazonas

Peru

Eouador

Panama

São Paulo

Ecuador

Mexico, Guatemala, Panama

\section{Colombia}

Costarica 
Poecilosoma Hübn.

Hübn., Vcrz. bek. Schmett. p. 126 (1826). - Butl., Journ. Línn. Soc. Lond., Zool. 12, p. 389, t. 27, f. 5 (1876). - Hamps., Cat. Lep. Phal. 1, p. 256 (1898).

Agerocha Hübn., Zutr. Exot. Schmett. 3, p. 10 (1827).

chrysis Hïbn., Zutr. Exot. Schmett. 2, p. 8, f. 211, 212

(1827). - Hamps., Cat. Lep. Phal. 1, p. 256, f. 111 (1898).

megaspila Walk., List Lep. Ins. Br. Mus. 31, p. 90 (1864) (Cosmosoma).

eone Hüibn., Zuir. Exot. Schmett. 3, p. 10, f. 417, 418

(1827) (Agerocha ). - Hamps., Cat. Lep. Phal. 1, 1. 257, f. 112 (1898).

eusebia Druce, Proc. Zool. Soc. Lond. p. 373 (1883) (Isanthrene). - Hamps., Cat. Lep. Phal. 1, p. 257 , t. 9, f. 18 (1898).

marginata(-1u1) Walk., List Lep. Ins. Br. Mus. 7, p. 1613, (1856) (Cosmosoma). - Hamps., Cat. Lep. Phal. 1, p. 258, t. 9, f. 19 (1898).

nigerrima Walk,, Iist Lep. Ins. Br. Mrs. 31, p. 86 (1864) (Gymnelia). - Hamps., Cat. Lep. Phal. 1, p. 256, t. 9, f. 17 (1898).

vespoldes Schaus, Proc. U. S. Nat. Mus. 29, p. 186 (1905). Peru

6

\section{Mystrocneme Herr.-Schäff.}

Herr.-Schäff., Ausscreur. Schmett. p. 21 (1856). - Hamps., Cat. Lep. Phal. 1, p. 258 (1898).

albicorpus Kaye, The Ent. 44, p. 143 (1911).

atavia Hamps., Cat. Lep. Phal. 1, p. 259, t. 9, f. 20 (1898). duleieordis Dyar, Journ. New York Ent. Soc. 15, p. 51 (1907).

flavieinetata Dogn., Hét. nouv. An. Sud 2, p. 6 (1911).

rubricorpus Kayc, The Ent. 44, p. 143 (1911).

sectum Kaye, The Ent. 44, p. 143 (1911).

varipes Walk., List Lop. Ins. Br. Mus. 1, p. 235 (1854) (Euchromia). - Hamps., Cat. Lep. Phal. 1, p. 258, f. 113 (1898). - Klages, Proc. U. S. Nat. Mus. 29, p. 537 (1906).

Amazonas

Amazonas,

Pará,

Santa

Catharina

(M.C.Vind.)

Ecuador

Amazonas

geminata Herr.-Schäff., Aussereur. Schmett. 1, 7 p. 72 , f. 419 (1856).

\section{Dixophlebia Butl.}

Butl., Journ. Linn. Soc. Lond., Zool. 12, p. 397, t. 28, f. 8 (1876). - Hamps.; Cat. Lep. Phal. 1, p. 259 (1898).

holophaea Hamps., Ann. \& Mag. Nat. Hist. (8) 4, p. 346 Brit.(1909).

Brit.-

Guayana

Amazonas

Mexico

Franz.-

Guayana

Brit.-

Guayana

,

Venezucla, Surinam,

Pará

Guayana 
quadristrigata Walk, List Lep. Ins. Br. Mus. 31, p. 82 (1864) (Pseudomya). - Hamps., Cat. Lep. Phal. 1, p. 259, f. 114 (1898).

2

Amazonas,

Minas

Geraës,

Santa

Catharina

(M.C.Vind.)

\section{Phoeniostaeta Hamps.}

Hamps., Cat. Lep. Phal. 1, p. 259 (1898).

Laematobasis (haematabasis) Hanps., Cat. Lep. Phal. 1, I p. 260, f. 115 (1898).

Santa

Catharina

\section{Iehoria Butl.}

Butl., Journ. Linn. Soc. Lond., Zool. 12, p. 370, t. 28, f. 9 (1876). - Hamps., Cat. Lep. Pha!. 1, p. 260 (1898).

Brieenoi Rothseh., Nov. Zool. 18, p. 36 (1911).

chaleomedusa Druee, Proe. Zool. Soe. Lond. p. 282 (1893). - Hamps., Cat. Lep. Phal. 1, p. 261, t. 10, f. 1 (1898).

chrostosomides Schaus, Proe. U. S. Nat. Mus. 29, p. 187 (1905).

deuon a Druee, Biol. Centr.-Am., Het. 2, p. 347, t. 71, f. 29 (1897) (Dycladia). - Hamps., Cat. Lep. Phal. 1, p. 260 (1898).

multigutta Schaus, Proe. Zool. Soe. Lond. p. 226 (1894) (Laemocharis). - Hamps., Cat. Lep. Phal. 1, p. 260, t. 9, f. 24 (1898).

quadrigutta Walk., List Lep. Ins. Br. Mus. 1, p. 245 (1854) (Euchromia). - Butl., Illustr. Het. Br. Mus. 1 , p. 25, t. 10, f. 1 (1877). - Druee, Biol. Centr.-Am., Het. 1, p. 47 (1884). - Hamps., Cat. Lep. Phal. 1, p. 261, f. 116 (1898).

semioprea Dogn., Ann. Soe. Ent. Belg. 50, p. 179 (1906). trieincta Herr.-Sehäff., Ausserenr. Selımett. 1, p. 74, f. 300 (1855) (Glaucopis). - Hamps., Cat. Lep. Plial. 1, p. 261 (1898).

Venezuela

Amazonas

(M.C.Vind.),

Santa

Catharina,

Paraná

Franz.-

Guayana

Mexieo

Venezuela

Mexico,

Guatemala,

Costariea

São Paulo

Colombia (MI.

C. Vind.),

São Paulo

(M.C.Vind.),

Rio Janeiro

8

Pseudomya Hübn.

Hübn., Verz. bek. Sehmett. p. 124 (1826). - Hamps., Cat. Lep. Phal. 1, p. 262 (1898).

Pseudocharis Druce, Biol. Centr.-Am., Het. 1, p. 56 (1884).

melauthus Cram., Pap. Exot. 4, p. 147, t. 367, f. C (1782) Colombia,

(Sphinx). - Hamps., Cat. Lep. Phal. 1, p. 264, Surinam, f. 117 (1898).

trigutta Walk., Iist Lep. Ins. Br. Mus. 1, p. 145 (1854) (Glaucopis). - Buti., Illustr. Het. Br. Brasilien

(ubi?)

IIus. 1 , p. 29 , t. 7 , f. 10 (1877).

(M.C.Vind.) 
miniua Grote, Proc. Ent. Soc. Philad. 6, p. 298, t. 3, f. 6 (1867) (Hippola). - Hamps., Cat. Lep. Phal. 1, p. 262, t. 10, f. 7 (1898). - Holl., Moth Book p. 99, t. 13, f. 6 (1903).

Biologie: Dyar, Psyche 8, p. 42 (1897). - Hamps., Cat. Lep. Plial. 1, p. 263 (1898).

naenia Druce, Biol. Centr.-Am., Het. 1, p. 57, t. 7, f. 12 (1884) (Pseudocharis). - Hamps., Cat. Lep. Phal. 1, p. 264 (1898).

nigrozona(-um) Schaus, Proc. U. S. Nat. Mus. 29, p. 187 (1905).

oelreiventris Dogn., Ann. Soc. Ent. Belg. 46, p. 227 (1902).

picta Schaus, Proc. Zool. Soc. Lond. p. 226 (1894). Hamps., Cat. Lep. Phal. 1, p. 263, t. 10, f. 24 (1898).

sanguiceps Hamps., Cat. Lep. Phal. 1, p. 264, t. 10, f. 4 (1898).

sithon Druce, Biol. Centr.-Am., Het. 1, p. 57, t. 7, f. 7 (1884); 2, p. 342 (1897) (Pseudocharis). Hamps., Cat. Lep. Phal. 1, p. 263 (1898).

splendens Druce, Ann. \& Mag. Nat. Hist. (6) 2, p. 240 (1888) (Mastigocera). - Hamps., Cat. Lep. Phal. 1, p. 262 , t. 10 , f. 2 (1898).

trabea Druce, Anm. \& Mag. Nat. Hist. (7) 3, p. 230 (1899).

trauslueida Dogn., Le Nat. (2) 4, p. 91 (1890) (Pseudocharis); Lcp. Loja p. 48 , t. 4, f. 2 (1891). 11 Hamps., Cat. Lep. Phal. 1, p. 264 (1898).

\section{Holophaea Hamps.}

Hamps., Cat. Lep. Phal. 1, p. 265 (1898).

eaerulea Druce, Ann. \& Mag. Nat. Hist. (7) 1, p. 408 (1898). - Hamps., Cat. Lep. Phal. 1, p. 266, t. 10, f. 3 (1898).

endoleuea Dogn., Aun. Soc. Ent. Belg. 53, p. 214 (1909). gentilicia Schaus, Ann. \& Mag. Nat. Hist. (8) 7, p. 178 (1911).

lugens Jones, Trans. Ent. Soc. Lond. p. 144 (1908).

lycone Druce, Biol. Centr.-Am., Het. 1, p. 47, t. 6, f. 9 (1884); 2, p. 335 (1896) (Ychoria). - Hamps., Cat. Lep. Phal. 1, p. 265, f. 118 (1898).

melita Druce, Ann. \& Mag. Nat. Hist. (7) 3, p. 230 (1899). prometina Druce, Ann. \& Mag. Nat. Hist. (6) 13, p. 172 (1894) (Thrinacia); Biol. Centr.-Am., Het. 2, p. 342 , t. 71 , f. 13 (1897). - Hamps., Cat. Lep. Phal. 1, p. 265 (1898).

ruatana Druce, Biol. Centr.-Am., Het. 2, p. 342, t. 71, f. 17 (1897) (Thrinacia). - Hamps., Cat. Lep. Plial. 1, p. 266, f. 119 (1898).

vesta Möschl., Verh. zool.-bot. Ges. Wien 27, p. 637, t. 8, f. 7 (1877) (Hysia). - Hamps., Cat. Lep. Phal. 1, p. 265 (1898).

Florida,

Cuba

Guatemala

Franz.Guayana

Ecuador

Venezuela

Panama

Mexico, Guatemala

Bahama-I., (New Providence)

Brit.Guayana, Ecuador

Ecuador

Ecuador

Colombia

Costarica

São Paulo

Mexico, Costarica Panama

Eeuador

Mexico

Honduras

(I. Ruatan)

Franz.-

Guagana,

Surinam,

Brasilien

(ubi?)

M. C. Vind.) 
Hypatia Kirby.

Kirby, Cat. Het. 1, p. 147 (1892). - Hamps., Cat. Lep. Phal. 1, p. 266 (1898). Hysia Walk., List Lep. Ins. Br. Mus. 1, p. 172 (1854) (nec Muls. 1851).

deleeta Butl., Journ. Linn. Soc. Lond., Zool. 12, p. 396 (1876) (Hysia). - Hamps., Cat. Lep. Phal. 1, p. 266, f. 120 (1898).

astyoche Walk., List Lep. Ins. Br. Mus. 1, p. 173

(1854) (Glaucopis) (nee Hübn.-Gey.).

melnleuen Walk., List Lep. Ins. Br. Mus. 1, p. 173 (1854)

(Glaucopis). - Butl., Illustr. Het. Br. Mus. 1,

p. 35, t. 11, f. 2 (1877). - Hamps., Cat. Lep. 2

Phal. 1, p. 267 (1898).

\section{Psilopleura Hamps.}

Hamps, Cat. Lep. Phal. 1, p. 267 (1898).

dolens Schaus, Ann. \& Mag. Nat. Hist. (8) 7, p. 178 (1911). hymenopteridia Rothsel., Nov. Zool. 18, p. 36 (1911). KRlagesi Rothsch., Nov. Zool. 18, p. 36 (1911).

Pentheri Zerny, Iris 26 (1912) (ined.).

polia Druce, Ann. \& Mag. Nat. Hist. (7) 1, p. 407 (1898). - Hamps., Cat. Lep. Phal. 1, p. 268, t. 10, f. 8 (1898).

sanguipuneta Hamps., Cat. Lep. Phal. 1, p. 268, t. 10, f. 6 (1898).

vittata Walk., Iist Lep. Ins. Br. Mus. 31, p. 83 (1864) (Pheia). - Druce, Biol. Centr.-Am., Het. 1, p. 61, t. 7, f.21 (1884); 2, p. 347 (1897). - Hamps., 7 Cat. Lep. Phal. 1, p. 267, f. 121 (1898).

\section{Pará}

Pará

\section{Costarica}

Bolivia

Venezuela

Bahia

Espiritu

Santo

Rio Grande do Sul

Mexico,

Guatemala

\section{Hhynchopyga Feld.}

Feld., Reise Nov., Lep. Atlas, p. 8 (1869). - Hamps., Cat. Lep. Phal. 1, p. 268 (1898).

braconida Kaye, The Ent. 44, p. 143 (1911).

flavicollis Druec, Biol. Centr.-Am., Het. 1, p. 46, t. 7, f. 11 (1884) (Amycles). - Hamps., Cat. Lep. Phal. 1, p. 270 (1898).

ielneumonea Fcld., Reise Nov. Lep. 2, p. 2, t. 102, f. 30 (1869). - Hamps., Cat. Lep. Phal. 1, p. 269, f. 122 (1898).

Meisteri Berg, An. Soe. Cient. Argent. 16, p.153 (1883) (Pooloptera). - Hamps., Cat. Lop. Phal. 1, p. 270 , t. 10 , f. 11 (1898).

Biologie: Sclirottky, Iris 22, p. 127-128 (1909) (Mimicry).

metaphaen Hamps., Cat. Lep. Phal. 1, p. 271, f. 124 (1898).

fla vicoll is Druce, Biol. Centr.-Am., Het. 2, p. 335 (1897) (Amycles).

rubricineta Hamps., Cat. Lep. Phal. 1, p. 269, t. 10, Bolivia

São Paulo

Guatemala

Colombia

Pará,

Paraná,

Paraguay,

Uruguay,

Argentinien

Mexico,

Honduras f. 10 (1898). 
semirufa Druec, Ann. \& Mag. Nat. Hist. (7) 18, p. 78 Peru (1906).

steniptera Hamps., Ann. \& Mag. Nat. Hist. (8) 4, p. 346 Brit. (1909).

Guayana

subflamma Druce, Biol. Centr.-Am., Het. 1,·p. 56, t. 8,

Panama f. 10 (1884) (Gymnopoda). - Hamps., Cat. Lep. Phal. 1, p. 270, f. 123 (1898).

xanthospila Hamps., Cat. Lep. Phal. 1, p. 269, t. 10, Bolivia 10 f. 9 (1898).

\section{Hypoclaaris Hamps.}

Hamps., Cat. Lep. Phal. 1, p. 271 (1898).

elusia Druee, Ann. \& Mag. Nat. Hist. (6) 20, p. 303 (1897) (Laemocharis). - Hamps., Cat. Lop. 1

Phal. 1, p. 271, f. 125 (1898).

Amazonas

\section{Saurita Herr.-Schäff.}

Herr.-Schäff., Aussercur. Sehmett. p. 21 (1854). - Hamps., Cat. Lep. Phal. 1, p. 271 (1898).

Gymnopoda Feld., Reise Nov., Lep. Atlas p. 5 (1869).

Echoneura Butl., Journ. Linn. Soe. Lond., Zool. 12, p. 383 (1876).

Thrinacia Butl., Journ. Linn. Soc. Lond., Zool. 12, p. 384 (1876).

Biologic: Seitz, Stett. Ent. Zoitg. 51, p. 262 (1890) (Mimicry).

anthracina Klages, Proc. U. S. Nat. Mus. 29, p. 537 (1906).

attenuata Hamps., Ann. \& Mag. Nat. Hist. (7) 15, p. 428

Venezucla (1905).

tenuis Butl., Illustr. Hct. Br. Mus. 1, p. 30, t. 17, f. 1 (1877) (Psendomya). - Hamps., Cat. Lep. Phal. 1, p. 273 (1898) (nec Butl. 1876).

astyoche Hübn.-Gey., Zutr. Exot. Schmett. 4, p. 41, f. 793, 794 (1832) (Euchromia). - Hamps., Cat. Lep. Phal. 1, p. 279 (1898).

bicolor Dogn., Ann. Soe. Fint. Belg. 46, p. 227 (1902).

bipuneta Hamps., Cat. Lep. Phal. 1, p. 280, t. 10, f. 27 (1898).

biradiata Feld., Rcise Nov., Lep. 2, p. 8, t. 102, f. 37 (1869) (Pseudomya). - Hamps., Cat. Lep. Phal. 1, p. 273 (1898).

cardinalis Rothsel., Nov. Zool. 18, p. 37 (1911).

cassandra L., Syst. Nat. ed. 10, p. 494 (1758) (Sphinx). Cram., Pap. Exot. 4, p. 218, t. 391, f. G (1782).

- Hübn., Samml. Exot. Sehmett. 1, t. 165 (1827).

- Sepp., Surin. Vlind. 2, p. 193 , t. 85 (1848).

- Hamps., Cat. Lep. Phal. 1, p. 274, f. 127 (1898). - Silva, Lep. Bras. p. 128, t. 23, f. 81 (1907).

Amazonas

Surinam, Rio Grande do Sul

Argentinien

Amazonas (M. C. Vind.), Santa

Catharina, Rio Grande do Sul, Paraguay, Argentinicn

Amazonas

Costariea

Trinidad, Venezuela, Surinam, Franz.Guayana (II. C. Vind.), 
Biologie: Sepp, Surin. Vlind. 2, p. 193, t. 85 (1848). - Schrottky, Iris 22, p. 124 (1909).

clandestina Zerny nom. nov.

cryptoleuca Hamps., Ann. \& Mag. Nat. Hist. (7)

I1, p. 340 (1903) (nec Walk. 1854).

concisa Walk., List Lep. Ius. Br. Mus. 1, p. 243 (1854) (Euchromia). - Hamps., Cat. Lep. Phal. 1, p. 279, t. 10, f. 19 (1898).

afflicta Druce, Biol. Cuntr.-Am., Het. 1, p. 56 (1884) (Thrinacia) (nec Walk.).

eryptoleuea Walk., List Lep. Ins. Br. Mus. 1, p. 239 (1854) (Euchromia). - Hamps., Cat. Lep. Phal. 1, p. 272, t. 10, f. 21 (1898).

diapliana Dogn., Ann. Soc. Fnt. Belg. 50, p. 179 (1906). diffusa Sehaus, Ann. \& Mag. Nat. Hist. (8) 7, p. 179 (1911). erythrogyia (-guia) Hamps., Cat. Lep. Phal. 1, p. 274, t. 10 , f. 5 (1898).

hamou Druce, Ann. \& Mag. Nat. Hist. (7) 9, p. 322 (1902). hemiphaea Dogn., Ann. Soc. Ent. Belg. 53, p. 215 (1909).

improvisa Schaus, Ann. \& Mag. Nat. Hist. (8) 9, p. 36 (1912).

incerta Walk., List Lop. Ins. Br. Mus. 7, p. 1627 (1856) (Tipulodes). - Hamps., Cat. Lep. Phal. I, p. 277 (1898).

corallonota Feld., Reise Nov., Lep. 2, p. 5, t. 102, f. 23 (1874) (Gymnopoda).

intermedia Druce, Biol. Centr.-Am., Het. 1, p. 56 (1884); 2, p. 342 (1897) (Thrinacia).

intrienta Walk., list Lep. Ins. Br. Mus. 1, p. 237 (1854) (Euchromia). - Hamps., Cat. Lep. Phal. 1, p. 280 , t. 10 , f. 26 (1898).

cata stibina Butl., Journ. Linn. Soc. Lond., Zool. 12, p. 384 (1876) (Echoneura).

ab. a ngusta Butl., Journ. Linn. Soc. Lond., Zool. 12, p. 384 (1876) (Echoneura). - Hamps., Cat. Lep. Phal. 1, p. 281 (1898).

lacteata Butl., Illustr. Hot. Br. Mus. 1, p. 34, t. 17, f. 3 (1877) (Dycladia). - Hamps., Cat. Lep. Phal. 1, p. 276, f. 128 (1898).

lasiophlebia (lasiphlebia) Dogn., Ann. Soe. Ent, Belg. 50, p. 180 (1906).

latens Seliaus, Ann. \& Mag. Nat. Hist. (8) 7, p. 178 (1911).

mecrida Druce, Ann. \& Mag. Nat. Hist. (6) 4, p. 84 (1859) (Gymnopoda); Biol. Centr.-Am., Het. 2, p. 342 , t. 71, f. 12 (1897). - Hamps., Cat. Lep. Phal. 1, p. 272 (1898).

mediorubra Kayc, The Ent. 44, p. 144 (1911).

melanifera Kayc, Tho Ent. 44, p. 144 (1911).

moru Druce, Biol, Centr.-Am., Het. 2 , p. 348 , t. 72 , f. 8 (1897) (Dycladia). - Hamps., Cat. Lep. Phal. 1, p. 275 (1898).

inyrrha Druee, Biol. Centr.-Am., Het. 1, p. 59, t. 7, f. 10 (1884) (Cosmosoma). - Hamps., Cat. Lep. Phal. 1, p. 278 (1898).

nigripalpla Hamps., Cat. Lep. Phal. 1, p. 274, t. 10, f. 20 (1898).

cassandra Druce, Biol. Centr.-Am., Het. 1, p. 46 (1884); 2, p. 355 (1896) (nec L.).
Paraguay, Argentinien

Rio Janeiro

Panama,

Pará

Venezuela, Pará

Peru

Costarica

São Paulo

(M.C.Vind.)

Peru

Franz.-

Guayana

Costarica

Panama,

Colombia

Rio Janeiro, Espiritu

Santo

Trinidad,

Amazonas

Peru

Costariea

Mexico

São Paulo

Panama

Guatemaia

Mexico,

Honduras,

Costarica 
nox Druee, Ann. \& Mag. Nat. Hist. (6) 18, p. 30 (1896) (Laemocharis); Biol. Centr.-Am., Het. 2, p. 341 , t. 71, f. 11 (1897). - Hamps., Cat. Lop. Phal. 1, p. 277 (1898).

ochracea Feld., Reise Nov., lep. 2, p. 5, t. 102, f. 22 (1869) (Gymnopoda). - Druec, Biol. Centr.-Am., Het. 2, p. 342 (1897). - Hamps., Cat. Lep. Phal. 1, 1. 272 (1898).

musca Sohaus, Proe. Zool. Soe. Lond. p. 275 (1892) (Pseudomya).

pontia Iruee, Ann. \& Mag. Nat. Hist. (6) 13, p. 172 (189.1) (Thrinacia).

pellucida Selıaus, Proc. Zool. Sue. Lond. p. 274 (1892) (Pseudomya); Ann. Lep. p. 4, t. 1, f. 4 (I892). verspicua Sehaus, Proc. U. S. Nat. Mus. 29, p. 187 (1905). phoenicosticta Hamps., Cat. Lep. Phal. 1, p. 277, t. 10, f. 17 (1898).

trigutta Druee, Biol. Centr.-Am., Het. 1, p. 55 (1884); 2, p. 341 (1897) (Laemocharis) (nee Walk.).

rubripuneta Selsaus, Ann. \& Mag. Nat. Hist. (8) 7, p. 178 (1911).

sulta Sehaus, Proc. Zool. Soe. Lond. p. 226 (1894) (Thrinacia). - Hamps., Cat. Lep. Phal. 1, p. 277, t. 10 , f. 12 (1898).

anguinea Druec, Biol. Centr.-Am., Het. 1, p. 56, t. 7 , f. 9 (1884) (Thrinacia). - Hamps., Cat. Lep. Phal. 1, p. 278 (1898).

sanguisecta Hamps., Cat. Lep. Phal. 1, p. 275, t. 10, f. $14(1898)$.

sericea Herr.Sohidf., Aussereur. Sehmett. p. 73, f. 253 (1854) (Glaucopis). - Druee, Biol. Centr.-Am., Het. 2, p. 335 (1896). - Hamps., Cat. Lep. Phal. 1, p. 279 (1898).

demoanassa Druce, Ann. \& Mag. Nat. Hist. (6) 18, p. 29 (1896) (Pseudosphenoptera); Biol. Centr.Am., Het. 2, p. 336, t. 71, f. 1 (1896).

submacula Sehaus, Ann. \& Mag. Nat. Hist. (8) 7, p. 179 (1911).

temenus Cram., Pap. Exot. 4, p. 148, t. 367, f. D (1782) $(\operatorname{Sphinx})$ - Hamps., Cat. Lop. Phal. 1, p. 279 (1898).

afflicta Walk., List Lep. Ins. Br. Mus. 1, p. 144 (1854) (Glaucopis). - Butl., Illustr. Het. Br. Mus. 1, p. 29, t. 7, f. 12 (1877).

tenuis Butl., Joun'n. Linn. Soe, Lond., Zool. 12, p. 384 (1876) (Echoneura). - Hamps., Cat. Lep. Plial. 1, p. 278 , t. 10, f. 18 (1898).

thoracica Klages, Proc. U. S. Nat. Mus. 29, p. 538 (1906). tijuea Sehaus, Proe. Zool. Soc. Lond. p. 275 (1892) (Pseridomya); Am. Lep. p. 4, t. 1, f. 3 (1892).

tlpullua Hübn., Samml. Exot. Sehmett. 1, t. 163 (1827) (Glaucopis). - Butl., Illustr. Het. Br. Mus. 1, p. 29, t. 7, f. 7 (1877). - Druee, Biol. Centr.-Am., Het. 1, p. 57 (1884); 2, p. 342 (1897). - Hamps., Cat. Iep. Phal. 1, p. 281 (1898).

bibia Walk., List Iep. Ins. Br. Mus. 1, p. 143 (1854) (Glaucopis).
Honduras, Venczuela

Mexico, Colombia

Rio Janciro

Trinidad

Guatemala,

Costarica

Costarica

Venezuela

Guatemala

Colombia

Panama,

Rio

Janeiro,

Argentinien

Costariea

Trinidad, Surinam, Pará

Espiritu Santo

Venezuela

Rio Janciro

Mexieo, Guatemala Panama, Pará, São Paulo, (M. C. Vind.), 
Biologie: Druce, Biol. Centr.-Am., Het. 1, p. 57 (1884) (Mimicry).

triangulifera Druce, Ann. \& Mag. Nat. Hist. (7) 1, p. 207 (1898) (Pseudosphenoplera). - Hanps., Cat. Lep. Phal. 1, p. 280, t. 10, f. 22 (1898).

tricolor Schaus, Proc. U. S. Nat. Mus. 29, p. 187 (1905).

tristissima Perty, Delect. Anim. Artic. p. 156, t. 31, f. 7 (1834) (Glaucopis). - Hamps., Cat. Lep. Phal. 1, p. 273 , f. $126(1898)$.

venezuelensis Klages, Proc. U. S. Nat. Mus. 29, p. 538 (1906).

ab. obscura Klnges, Proc. U. S. Mus. 29, p. 538 (1906).

vludonissa Druec, Proc. Zool. Soc. Lond. p. 378 (1883) (Dycladia). - Hamps., Cat. Lep. Phal. 1, p. 278, t. 10, f. 15 (1898).

vitristriga Druce, Ann. \& Mag. Nat. Hist. (6) 20, p. 301 (1897). - Hamps., Cat. Lep. Phal. 1, p. 280, t. 10, f. 25 (1898). - Klages, Proc. U. S. Nat. Mus. 29, p. 537 (1906).

Watsonl Rothsch., Nov. Zool. 18, p. 37 (1911). 51

Espiritu

Santo,

Santa

Catharina

Amazonas,

Sז̃o Paulo

Franz.-

Guayana

Pará

'Venezuela

Ecuador

Venezuela,

Brit.-

Guajana

Panama

\section{Sauritinia Dyar.}

Dyar, Proc. U. S. Nat. Mus. 29, p. 173 (1905).

dubiosa Schaus, Proc. U. S. Nat. Mus. 29, p. 188 (1905). Franz.-

1

Guayana

\section{Scena Walk.}

Walk., List Lep. Ins. Br. Mus. 1, p. 263 (1854). - Hamps., Cat. Lep. Phal. 1, p. 281 (1898).

potentia Druce, Ann. \& Mag. Nat. Hist. (6) 13, p. 171 Mexico,

(1894) (Chloropsinus); Biol. Centr.-Am., Het. 2, Costarica

p. 351, t. 72, f. 12 (1897). - Hamps., Cat. Lep. Phal. 1, p. 281, f. 129 (1898).

styx Walk., List Lep. Ins. Br. Mus. 1, p. 264 (1854) Mexico

(Euchromia). - Hamps., Cat. Lep. Phal. 1, p. 282 , f. 130 (1898).

propylea Druce, Ann. \& Mag. Nat. Hist. (6) 13,

p. 170 (1894); Biol. Centr.-Am., Het. 2, p. 334, 2

t. 70 , f. 26 (1896).

\section{Mieragyrta Butl.}

Butl., Cist. Ent. 2, p. 114 (1870). - Hamps., Cat. Lep. Phal. 1; p. 282 (1898).

diminuta Walk., List Lep. Ins. Br. Mus. 2, p. 328 (1854) Amazonas, (Dioptis). - Hamps., Cat. Lep. Plial. 1, p. 283, f. 131 (1898).

gavisa Walk., List Lop. Ins. Br. Mus. 31, p. 147 1 (1864) (Agyrta). 
Metaloba Hamps.

Hamps., Cat. Lep. Phal. 1, p. 283 (1898).

argante Druce, Ann. \& Mag. Nat. Hist. (6) 20, p. 301 (1897) (Calonotos). - Hamps., Cat. Lep. Phal. 1, p. 284 , t. 10, f. 13 (1898).

nana Druce, Ann. \& Mag. Nit. Hist. (6) 20, p. 300 (1897) (Calonotos). - Hamps., Cat. Lep. Phal. 1, 2 p. 283, f. $132(1898)$.

Eeuador

Colombia

\section{Psoloptera Butl.}

Butl., Juurn. Linn. Soc. Lond., Zool. 12, p. 369 (1876). - Möschl., Verh. zool.-Jot. Ges. Wien 27, p. 633 (1877). - Hamps., Cat. Lep. Plial. 1, p. 284 (1898).

aurifera Herr.-Schäff., Aussereur. Schmett. p. 72, f. 252 (1854) (Copaena ). - Hamps., Cat. l.ep. Phal. 1, p. 285 (1898).

basifulva Schaus, Proc. Zool. Soc. Lond. p. 225 (1894). - Hamps., Cat. Lep. Plial. 1, p. 284, t. 10, f. 23 (189S).

thoracica Druce, Biol. Centr.-Am., Het. 1, p. 46 (1884) (pr. p.).

lencosticta Hülın., Sinuml. Exot. Schmett. 1, t. 162 (1827) (Glaucopis). - Hamps., Cat. Lep. Phal. 1, p. 285 (1898).

thoraciea Walk., List Lep. Ins. Br. Mns. 1, p. 243 (1854) (Euclercmia). - Butl., Illustr. Het. Br. Mus. 1, p. 24, t. 8, f. 6 (1877). - Druce, Biol. Centr.-Am., Het. 1, p. 46 (1884) (pr. p.). - Hamps., Cat. 4 Lep. Phal. 1, p. 285, f. 133 (1898).

Amazonas (M.C.Vind.), São Paulo

Panama, Peru

Venezuela, Surinam, Pará Guatemala, Panama, Ecuador Amazonas

\section{Eurota Walk.}

Walk., List Lep. Ins. Br. Mus. 1, p. 180 (1854). - Burm., Descr. Phys. Rep. Argent. 5, p. 377 (1878). - Hamps., Cat. Lep. Phal. 1, p. 285 (1898).

Endera Walk., List Lep. Ins. Br. Mus. 1, p. 228 (1854).

Fregella Walk., List Lep. Ins. Br. Mus. 1, p. 272 (18i5)

Baeri Rothsch., Nov. Zool. 1S, p. 37 (1911).

elegaus Druce, Aun. \& Mag. Nat. Hist. (7) 18, p. 78 (1906).

helena Hel'.-Schäff., Ansscreur. Schmictt. 1). 73, f. 230 (1854) (Claucopis). - Hamps., Cat. Lep. Phal. 1, p. 291 (1898).

laetifica Manss. in Stübel's Reise Südum., Lep. p. 129 , t. 4, f. 19 (1890) (Laemocharis).

hermione Burm., Mescr. Phys. Rep. Argent. 5, p. 380 (1878). - Hamps., Cat. Iep. Phal. 1, p. 286 , f. 134 (1898). - Schrottky, Tris 24, p. 150 (1911). v. paraguayensis Sclirottky, Iris 24, p. 150 (1910). Biolugic: Schrottky, Iris 24, p. 1 bi (1910).

Herrichi (Herrickii) Butl., Journ. Linn. Soc. Lond., Zool. 12, p. 366 (1876). - Berg, An. Soc. Cient. Argent. 13, p. 178 (1882). - Hamps., Cat. Lep. Phal. 1, p. 287 (1898).
Argentinien

Paraguay

Bolivia

Rio Grande do Sul, Argentinien

Argentinien, Uruguay (M.C.Vind.)

Paraguay

Paraguay, Argentinien 
sericaria Herr.-Schäff., Aussereur. Schmett. p. 73, f. 229 (1854) (Glaucopis) (nec Perty). - Burm., Descr. Pliys. Rep. Argent. 5, p. 378 (1878).

histrio Guér., Icon. Régne Animal, Ins. "p. 502 (1843) (Glaucopis). - Burm., Descr. Phys. Rep. Argent. 5, p. 381 (1878). - Hamps., Cat. Lep. Phal. 1, p. 289 , t. 10 , f. 28 (159S).

igniventris Burm., Descr. Phys. Rep. Argent. 5, p. 378 (1878). - Hamps. Cat. Lep. Plıal. 1, p. 289 (1898). Inaritana Schaus, Joun. New York Ent. Soc. 4, p. 130 (1896). - Hamps., Cat. Lep. Hhal. 1, p. 290, t. 11, f. 16 (1898).

minerva Sehaus, Journ. Now York Ent. Soc. 9, p. 41 (1901).

nigrieincta Hamps., Ann. \& Mag. Nat. Hist. (7) 19 , p. 226 (1907).

Parishi Rothsch., Nov. Zool. 18, p. 37 (1911).

patagiata Burm., Descr. Phys. Rep. Argent. 5, p. 379 (1878). - Hamps., Cat. Lep. Phal. 1, p. 290 (1898).

picta Herr.-Schäff., Ausscreur. Schmett. p. 73, f. 52 (1853) (Glaucopis). - Butl., Illustr. Het. Br. Mus. 1, p. 22, t. 11, f. 14 (1877). - Hamps., Cat. Lep. Phisl. I, p. 290 (1898).

pictula Walk., List lep. Ins. Br. Mus. 1, p. 181 (1854) (Glaucopis).

Schausi Haups., Cat. Lep. Phal. 1, p. 286, t. 10, f. 16 (1898).

helena Schals, Journ. New York Ent. Soc. 4, p. 130 (1896) (ncc, Herr.-Schäff.).

selva Herr.-Schäff., Aussercur. Schmett. p. 73, f. 227 (1854) (Glaucopis). - Burm., Deser. Phys. Rep. Argent. 5, p. 380 (1878). - Hamps., Cat. Lep. Phal. 1, p. 287 (1598).

semlluna Walk., List Lep. Ins. Br. Mus. I, p. 272 (185t) (Euchromia). - Butl., Illustr. Het. Br. Mus. 1, p. 22 , t. 9, f. 8 (1877). - Hamps., Cat. Lep. Phal. 1, p. 287 (1898).

sericaria Perty, Dolect. Anim. Artic. p. 159, t. 31, f. 14 (1834) (Glaucopis). - Herr.-S’chäff., Ausscieur. Schmett. p. 73, f. 229 (1854). — Hamps., Cat. Lep. Phal. I, p. 288 (1898).

stictibasis Hamps., Cat. Lep. Plial. 1, p. 290, t. 11, f. 1 (1898).

strigiventris Gućr., Voy. Coquillo, Zool. 2 (2), p. 283, t. 19, f. 8 (1830) (Giaucopis). - Burin., Descr. Phys. Rep. Argent. 5, p. 381 (1878). - Hamps., Cat. Lep. Phal. 1, p. 291 (1898).

tisamena Dogn., Ann. Soc. Ent. Belg. 46, p. 227 (1902). rulcanus Walk., List Lep. Ins. Br. Mus. I, p. 228 (1854) (Euchromia). - Hcrr.-Schäff., Aussereur. Schmett. p. 73, f. 295 (1854). - Druce, Biol. Centr.-Am., Het. I, p. 43 (1884); 2, p. 333 (1896). - Hamps., Cat. Lep. Phal. 1, p. 298, f. 135 (1898). xanthosolna Hamps., Ann. \& Mag. Nat. Hist. (7) 15, p. 428 (1905).

Bolivia,

São Paulo,

Paraguay

Argentinien

Paraná

Argentinien

Cubà

Argentinien

Venezucla, São Paulo (M.C.Vind.), Matto

Grosso (M.C.Vind.), Paraguay (M. C. Vind.)

Paraná

Paraguay

(M.C.Vind.),

Uruguay,

Argentiuien

Brasilien

(ubi?)

Minas Gcraës, Rio Janeiro

São Paulo

Sïdbrasilien, Paraguay, Argentinicn, Bolivia São Paulo Mexico

Argentinien 22 


\section{Dyeladin Feld.}

Feld., Reise Nov., Lep. Atlas p. 4 (1874). - Hamps., Cat. Lep. Phal. 1, p. 292 (1898).

correbioides Feld., Reise Nov., Lep. 2, p. 4, t. 102, f. 20

(1869). - Druce, Biol. Centr.-Am., Het. I, p. 61 (1884); 2, p. 347 (1897). - Hamps., Cat. Lep. Phal. 1, p. 293, f. 136 (1898).

lucetius Cram., Pap. Exot. 4, p. 129, t. 357, f. D (1782) (Sphinx). - Herr.-Schäff., Aussereur. Schmett. p. 73, f. 296 (1855). - Hamps., Cat. Lep. Phal. I, p. 292 (1898).

transiens Walk., List Lep. Ins. Br. Mus. 7, p. 1615 (1856) (Eurota).

lydia Druce, Ann. \& Mlag. Nat. Hist. (7) 6, p. 64 (1900). melaena Hamps., Cat. Lep. Phal. 1, p. 292, t. 11, f. 8 (1898).

vitrina Rothsch., Nov. Zool. 18, p. 37 (1911).

xantlobasis Hamps., Ann. \& Mag. Nat. Hist. (8) 4, p. 346 (1909).

Mexico,

Guatemala, Honduras,

Costarica,

Panama,

Colombia

Pará,

São Paulo,

Paraguay

(M.C.Vind.)

Südbrasilien

Bolivia,

São Paulo

(M.C.Vind.),

Santa

Catharina

Honduras, Ecuador 6

Brit.-

Guayana

\section{Euchromia Hiibn.}

Hübn., Verz. bok. Schmett. p. 121 (1826). - Butl., Journ. Linn. Soc. Lond., Zool. 12, p. 363, t. 28, f. 20 (1876). - Mcyr., Proc. Linn. Soc. New South Wales (2) 1, p. 786 (1886). - Hamps., Moths Ind. 1, p. 226 (1892); Cat. Lep. Phal. 1, p. 293 (189S). - Picp. \& Snell. Tijdschr. v. Ent. 47, p. 60 (1904). - Turner, Proc. Linn. Soc. New South Wales 29, p. 857 (1905).

Phalanna Walk., List Lep. Ins. Br. Mus. 1, p. 218 (1854).

Hira Walk., List Lep. Ins. Br. Alus. 1, p. 220 (1854).

\section{a) Äthiopische Arten.}

- amoena Möschl., Stett. Ent. Zcitg. 33, p. 350 (1872) (Phalanna). - Hamps., Moths Ind. 1, p. 228 (1892); Cat. Lep. Phal. 1, p. 295, t. II, f. 2 (1898). a rica na Butl., Journ. Linn. Soc. Lond., Zool. 12, p. 361 (1876).

Biologie: Barrett, Ent. Monthl. MIog. (2) 12, p. $193(1901)$.

formosa Gućr., Icon. Régne Animal, Ins. p. 501, t. 81 bis, f. 10 (1843) (Glaucopis). - Boisd., Faune Madag. p. 82, t. 11, f. 3 (1833). - Wallengr., Svenska Akad. Handl. 5 (4), p. 10 (1865). Hamps., Cat. Lep. Phal. 1, p. 295 (1898).

Deutsch- u.

Brit.-

Ostafrika,

Delagoabai,

Natal,

Zululand,

Transvaal,

Madagaskar

(MI.C.Vind.)

Sierra Leone

(M.C.Vind.),

Brit.- u.

Deutsch-

Ostafrika, 
Folletii Gray, Griff. Anim. Kingd, 15, t. 120, f. 10 (1833) (Glaucopis).

guineensis Fabr., Syst. Ent. p. 551 (1775) (Zygaena). - Auriv., Ent. Tidskr. 18, p. 153 (1897); Ark. Zool. 2 (4), p. 33 (1904).

ab. sperchia (-us) Cram., Pap. Exot. 2, p. 79, t. 146, f. C (1777) (Sphinx). - Hamps., Cat. Lep. Phal. p. 296 (1898) (f. prineip.).

inierstans Walk., List Lep. Ins. Br. Mus. 1, p. 221 (1854).

leonis Butl., Journ. Linn. Soc. Lond., Zool. 12, p. 363 (1876); Illustr. Het. Br. Mus. 1, p. 22, t. 10 , f. 2 (1877).

bellula Mab., Ann. Soe. Ent. Franec (6) 10, p. 36 (1890).

ab. discifera Zerny, Iris 26 (1912) (ined.).

ab. interrupta Grünberg, Sitzungsber. Ges. uaturf. Fi. Berlin, p. 176 (1910).

v. splendens Butl., Trans. Ent. Soe. Lond. p. 113, t. 4, f. 4 (1888). - Hamps., Cat. Lep. Phal. 1, p. 297 (1898).

Jacksoni Beth.-Baker, Ann. \& Mag. Nat. Hist. (8) 7 p. 531 (1911).

- letle Fabr., Syst. Ent. p. 553 (1775) Zygaena). - Hamps., Cat. Lep. Phal. 1, p. 296 (1898).

eumolphus Cram., Pap. Exot. 3, p. 8, t. 197, f. D (1779) (Sphinx).

fulvida Butl., Trans. Ent. Soe. Lond. p. 112, t. 4, f. 5 (1888).

Biologie: Sehaus \& Clements, Lep. Sierra Leone p. 22 (1893). - Auriv., Ark. Zool. 2 (4), p. 32, f. 28 (1904).

madagascariensis Boisd., Faune Madag. p. 83, t. 11, f. 4 (1833) (Glaucopis). - ? Wallengr., Svenska Akad. Handl. (5) 4, p. 10 (1865). - Hamps., Cat. Lep. Phal. 1, p. 294 (1898).

\section{-}

\section{b) Orientalische Arten.}

Horsfieldi Moore, Proe. Zool. Soe. Lond. p. 200, t. 60, f. 13 (1859) (Phalanna). - Hamps., Cat. Lep. Plial. 1, P. 297 (1898). - Sncll., Tijdsehr. v. Ent. 47, p. 61 (1904).

Biologie: Moore, Cat. lep. Wast Ind. Comp. 2, p. 329 , t. 14, f. 9, 9a (1859). - Hamps., Cat. Lep. Phal. 1, p. 297 (1898). - Piep. \& Snell., Tijdsehr. v. Ent. 47 , p. 62 , t. 10 , f. 13 (1904).

Bomeo,

Sumatra,

Java,

Bawean,

Bali,

Sumbawa,

Flores,

Christmas -

I. (Hamps.)

bique,

Natal,

Tapland,

Komoren,

Seychellen

C. Vind.)

bis Congo

Congo

Kamerun,

Alt-Calabar

Uganda

Senegam-

Congo $u$.

gola,

Po,

I. do

Principe,

(M.C.Vind.)

Madagaskar,

Tatal

(Wallengr.) 
magna Swinh., Trans. Ent. Soc. Lond. p. 133, t. 8, f. 1 (1891) (Syntomis). - Hamps., Moths Ind. 1, p. 227 (1892); Cat. Lcp. Phal. 1, p. 305 (1898).

- polymena L., Syst. Nat. ed. 10, p. 494 (1858) (Sphinx). - Drury, Ill. Exot. Ent. 1, p. 55, t. 26, f. 1 (1770). - Cram., Pap. Exot. 1, p. 20, t. 13, f. D (1775). - Lefebvre, Ann. Soc. Ent. France 11, p. 24, t. 11, f. 4 (1842). - Moore, Lep. Ceyl. 2, p. 39 , t. 9., f. 6, 6a (1882). - Meyr., Proc. Linn. Soc. Now South Wales (2) 1, p. 787 (1886). Hamps., Moths Ind. 1, p. 227, f. 143 (1892); Cat. Lep. Phal. 1, p. 297, f. $137^{\circ}$ (1898). - 'Turner, Proc. Linn. Soc. Now South Wales 29, p. 858 (1905).

v. celebensis Butl., Journ. Linn. Soc. Lond., Zool. 12, p. 364 (1876). - Hamps., Cat. Lep. Phal. 1, p. 298 (1898).

Butleri Röber, Iris 1, p. 202, 339 (1887).

v. diffusihelvola Sehultze, Philipp. Journ. Sc. (Gen. Sc.) 3, p. 29, t. I, f. 3 (I908).

v. elegantissima Wallengr., Lop. Eug. Resa p. 360 , (1861). - Semp., Schmott. Philipp. 2, p. 424 (1898).

orientalis Butl., Journ. Linn. Soc. Lond., Zool. 12, p. 364 (1876); Trans. Ent. Soc. Lond. p. 114, t. 4, f. 6 (1888). - Hamps., Moths lnd. 1, p. 227 (1S92); Cat. Lep. Phal. 1, p. 298 (1898). ab. formosana Butl., Trans. Ent. Soc. Lond. p. 114, t. 4, f. 7 (1888). - Hamps., Cat. Lep. Phal. 1, p. 298 (1898).

ab. fraterna Butl., Journ. Linn. Soc. Lond., Zool.12, p. 364 (1876). - Hamps., Cat. Lep. Phal. 1, p. 298 (18:9).

ab. laura Butl., Journ. Linn. Soc. Lond., Zool. 12, p. 364 (1876). - Hamps., Moths Ind. 1, p. 228 (1892); Cat. Lop. Phal. 1, p. 298 (1898).

ab. siamensis Butl., Journ. Linn. Soc. Lond., Zool. 12, p. 365 (1876). - Hanps., Cat. Lep. Phal. 1, p. 208 (1898).

Biologie: Mooro, Cat. Lop. East Ind. Comp. 2, p. 328 , t. 4, f. 8, 8a, b (1859); Lop. Coyl. 2, p. 39, t. 94, f. 6a (1882) (polymona). - Semp., Verh. zool.-bot. Ges. Wien 17, p. 701 (1867); Sclimett. Philipp. 2, p. 424, t. K, f. 3-5 (IS98) (elegantissima). - Hamps., Moths Ind. 1, p. 209, f. 136 , p. 227 (1892); Cat. Lep. Phal. 1, p. 22, f. 7, p. 298 (189S) (polymena).

\section{c) Australische Arten.}

aemulina Butl., Proe. Zool. Soc. Lond. p. 473 (1877). - Hamps., Cat. Lep. Plial. 1, p. 300, t. 11, f. $18(1898)$.

amboinica Hamps., Cat. Lep. Phal. 1, p. 302, t. 11, f. 6 (1898).

auranticincta Hamps., Cat. Lep. Phal. 1, p. 304, t. 11, f. 22 (1898).
Südindien :

Canara,

Ceylon

Bombay bis

Burma,

Ceylon,

Andama-

nen,

Negros,

Cebu,

Sangir,

Cclebes,

Ternate,

? Nord-

australien

Celebes

Luzon

Ccylon (MI.

C. Vind.),

Burma,

Siam,

Malakka,

Java (M.

C. Vind.),

Formosa,

Philippinen

Neuguinea

(ubi ?)

Amboina

Neuguinea (ubi?) 
brillantina Rothsch., Nov. Zool. 18, p. 38 (1911).

burien (bourica) Boisd., Voy. Astrolabe, Lép. p. 194, (1832) (Glaucopis). - Hamps., Cat. Lep. Phal. 1, p. 302 , t. 11 , f. 21 (1898).

coelipennis Walk., List Lep. Ins. Br. Mus. 31, p. 99 (1864) (Hira).

Pagensteeheri Röber, Iris 1, p. 201, 338, t. 7, f. 10 (1887) (Glaucopis).

caelipunctata Lathy, The Iint. 32, p. 117 (1899).

cincta Montr., Ann. Soe. Linn. Lyon 11, p. 252 (186t) (Glaucopis) ). - Hamps., Cat. Lep. Phal. 1, p. 300 (1898).

collaris Swinh., Ann. \& Mag. Nat. Hist. (7) 15, p. 496 (1905).

creusa L., Syst. Nat. ed. 10, p. 494 (1758) (Sphinx). - Clerek, Ioon. Ins. rar. t. 46, f. 5 (1759-64). - Boisd., Mon. Zyg. p. 121, t. 7, f. 7 (1829). - Hamps., Cat. Lep. Phal. 1, p. 304 (1898). - Turner, Proc. Linn. Soc. New South Wales 29, p. 858 (1905).

thelebas Cram., Pap. Exot. 2, p. 86, t. 150, f. D (1777) (Sphinx).

irus Gram., Pap. Exot. 4, p. 150, t. 368, f. A (1782) (Sphinx). - Meyr., Proc. Linn. Soc. New Sontl Wales (2) 1, p. 787 (1886).

ganymede Dbld. in Stokes, Austral. 1, p. 519, t: 3, f. 3 (1846) (Glaucopis).

cyanilis Meyr., Trans. Ent. Soc. Lond. p. 457 (1889). Hamps., Cat. Lop. Phal. 1, p. 301, t. 11, f. 5 (1898).

Dohertyi Druce, Ann. \& Nag. Nat. Hist. (7) 3, p. 230 (1899).

dubia Röber, Iris 1, p. 201, 339, t. 7, f. 12 (1887) (Glaucopis). - Hamps., Cat. Lep. Phal. 1, p. 302, t. 11 , f. 20 (1898).

Ekcikei Beth.-Baker, Nov. Zool. 15, p. 181 (1908).

epa Beth.-Baker, Nov. Zool. 15, p. 180 (1908).

Iulgens Lathy, The Ent. 32, p. 117 (1899).

gemmunta Butl., Ann. \& Mag. Nat. Hist. (5) 19, p. 216 (1887). - Hamps., Cat. Lep. Phal. 1, p. 299 , t. 11 , f. 17 (1898).

Horsfieldi Moore (ef. p. 87).

irius Boisd., Voy. Astrolabe, Lép. p. 192, t. 5, f. 8 (1832) (Glaucopis). - Meyr., Proc. Linn. Soc. New South Wales (2) 1, p. 788 (1886). - Hamps., Cat. Lep. Phal. 1, p. 303 (1898). - Turner, Proe. Linn. Soe. New South Wales 29, p. 858 (1905).
Buru

Amboina,

Ceram,

Buru

Buru

Neu-

kaledonien

Salomonen: Shortland

Celebes bis

Neusüd-

wales und

Neu-

hebriden,

Fidij-I.

(MI.C. Vind.)

Neuguinea

(ubi?)

Buru

Ceram,

Buru

(M.C.Vind.)

Brit.-

Neuguinea

I. Mafor b.

Holl. -

Neuguinea

Halmaheira

(M.C.Vind.),

Salomonen:

Alu, Lifu

(M. C. Vind

Macroma-

layana,

Sumbatwa,

Flores,

Christmas-

I. (Hamps.)

Molukken,

Aru-I.,

Mysol,

Holl. - und

Deutsch-

Nouguinea 
Boisduvalii Montr., Ann. Soc. Sc. Phys. Lyon (2) 8, p. 409 (1856) (Glaucopis).

a ruica Walk., List Iop. Ins. Br. Nus. 31, p. 98 (1864) (Hira).

isis Boisd., Voy. Astrolabe, Lép. p. 193 (1832) (Glaucopis). - Hamps., Cat. Lep. Phal. 1, p. 301, t. 11, f. 4 (1898).

Iurlina Butl., Trans. Ent. Soc. Lond., p. 110 (1858). Hamps., Cat. Lcp. Phal. 1, p. 301, t. 11, f. 19 (1898). - Turner, Proc. Linn. Soc. Netw South Wales 29, p. 859 (1905).

Mathewi Butl., Trans. Ent. Soc. Lond. p. 111, t. 4, f. 3 (1888). - Himps., Cat. Lcp. Phal. 1, p. 299 (1898). negleeta Rothsch., Nov. Zool. 18, p. 38 (1911).

v. occidentalis Rothsch., Nov. Zool. 18, p. 38 (1911).

oenone Butl., Journ. Linn. Soc. Lond., Zool. 12, p. 365 (1876); Trans. Ent. Soc. Lond. p. IIl, t. 4, f. 2 (1888). - Hamps., Cat. Lep. Phal. 1, p. 299 (1895).

paula Röber, Iris 1, p. 202, 339, t. 7, f. 11 (1887) (Glau. copis). - Hamps., Cat. Lep. Phal. 1, p. 303, t. 17 , f. 8 (1898).

pelewalla Swinh., Ann. \& Mag. Nat. Hist. (7) 18, p. 403 (1906).

plagosa Swinh., Ann. \& Mag. Nat. Hist. (7) 15, p. 495 (1905).

polymena I. (cf. p. 88).

v. celebensis Butl. (cf. p. 88).

Pratti Beth.-Baker, Nov. Zool. 15, p. 181 (1908).

rubricollis Walk., List Lep. Ins. Br. Mus. 31. p. 99 (1864) (Hira). - Hamps., Cat. Lep. Phal. 1, 1. 300 , t. 11 , f. 3 (1898).

Salomonis Swinh., Ann. \& Mag. Nat. Hist. (7) 15, p. 496 (1905).

shortlandiea Swinl., Ann. \& Mag. Nat. Hist. (7) 15, p. $496(1905)$.

vitiensis Hamps., Ann. \& Mag. Nat. Hist. (7) 11, p. 340 (1903).

(M.C.Vind.), Trobriand-

I.,

Cap York

Duke of York-I.,

Bismarck.

Archipel

Fergusson-I. ThursdayI.

Salomonen

Salomoncn :

Florida,

Guadal-

canar,

Bougainville, Isabel

Salomonen:

Vella-La.

Vella,

Kulam-

bangra

Salomonen:

Alu,

Malaita,

SantaAnna,

Guadal-

cinar

Celebes

Karolinen

Neupommern

Bombay bis

Philippi-

nen, Sangir,

Celcbes,

Ternate,

? Nordaustralien

Celebes

Brit.-

Neuguinea

Neuhebriden,

Lifu (M. C.

Vind.),

Salomonen

Salomonen:

Isabcl

Salomonen;

Shortland

Fidji-I. 
Wahuesi Rothsch., Nov. Zool. 18, p. 38 (1911).

Walkeri Hamps., Cat. Lep. Phal. 1, p. 303, t. 11, f. 7 $(1898)$.

Deutsch. Neuguinea Tornate, Batjan (M. C. Vind.), Obi (M. C. 41 Vind.)

\section{Syntonueida Harr.}

Harr., Am. Junrı. Sc. 36, p. 316 (1839). - Butl., Journ. Linn. Soc. Lond., Zool. 12, p. 366, t. 28, f. 16 (1876). - Möschl., Verh. zool.-bot. Ges. Wien 27, p. 633 (1877). - Hamps., Cat. Lep. Phal. 1, p. 305 (1898).

Hi ppola Walk., List Lep. Ins. Br. Mus. 1, p. 222 (1854).

a ustera Dogn., Arn. Soc. Ent. Belg. 46, p. 228 (1902). befana Skinner, Lint. Notws 17, p. 379 (1906).

epilais Walk., List Lep. Ins. Br. Mus. 1, p. 227 (1854) (Euchromia). - Butl., Illustr. Het. Br. Mus. 1, p. 23 , t. 8 , f. 5 (1877). - Druce, Biol. Centr.Am., Het. 1, p. 43 (1884); 2, p. 333 (1896). Hamps., Cat. Lep. Phal. 1, p. 308 (1898).

v. jucundissima Dyar, Journ. New York Ent. Soc. 15 , p. $226(1907)$.

epilais Ncum. \& Dyar, Journ. New York Ent. Soc. 1, p. 101 (1893). - Holl., Moth Book, p. 99 , t. 13 , f. 2 (1903).

Biologie: Dyar, Journ. Netw York Ent. Soc. 4, p. 72 , t. 3, f. 10 (1896); Ins. Life 2, p. 360 (1890); Proc. Boston Soc. Nat. Hist. 27, p. 138 (1863). - Hamps., Cat. Lop. Phul. 1, p. 308 (1898).

Hampsoui Barncs, Can. Ent. 36, p. 165 (1904).

ipomeae Harr., Am. Journ. Sc. 36, p. 316 (1839) (Glaucopis). - Noum. \& Dyar, Journ. New Yurk Ent. Soc. 1, p. 101 (1893). - Hamps., Cat. Lep. Phal. 1, p. 307, f. 139 (1898). - Holl., Moth Book p. 99, t. 13, f. 3 (1903).

ferox Walk., List Lep. Ins. Br. Mus. 1, p. 223 (1854) (Euchromia). - Butl., Illustr. Het. Br. Mus. 1, p. 23 , t. 10, f. 7 (1877).

euter pe Herr.-Schäff., Ausscreur. Schmett. p. 73, f. 430 (1856) (Glaucopis).

Biologic: Dyar, Proc. Boston Soc. Nat. Hist. 27, p. 138 (1896).

joda Druce, Biol. Centr.-Am., Hct. 2, p. 333, t. 71, f. 15 (1896). - Hamps., Cat. Lep. Plial. 1, p. 305 (1898).

melantlius Gram., Pap. Exot. 3, p. 94, t. 248, f. C (1780) (Sphinx). - Herr.-Schäff., Aussorcur. Schmett. p. 73, f. 228 (1854). - Möschl., Verh. zool.-bot. Gos. Wien 27, p. 633 (1877). - Dreue, Biol. Centr.-Am., Het. 1, p. 42 (1884); 2, p. 332 (1896). - Hamps., Cat. Lep. Phal. 1, p. 306, f. 138 (1898).

apricans Walk., List Lep. Ins. Br. Mus. 1, p. 224 (1854) (Euchromia).

ab. nycleus Cram., Pap. Exot. 4, p. 74, t. 325, f. $\quad$ (1780) (Sphinx). - Hamps., Cat. Lep. Phal. 1, p. 306 (1898).

\section{Paraguay \\ Arizona \\ Mexico, \\ Brit.- \\ Honduras, \\ Honduras}

Florida

Arizona

Süidl. Vereinigte

Staaten

Mexico

Mexico bis Panama, Venezuela, Surinam, Bahia (N) C. Vind.),

Uruguay

(M. C. Vind.), Peru 
ab. albifasciata Butl., Journ. Linn. Soc. Lond., Zool. 12, p. 366 (1876). - Druce, Biol. Centr. -Am., Het. 1, p. 42 (1884). - Hamps., Cat. Lep. Phal. 1, p. 306 (1898).

syntomoides Boisd., Spéc, Gén. Lép. I, t. 16, f. 4 (1836) (Glaucopis). - Himps., Cat. Lep. Phal. 1, p. 306 (1898).

Saulcyi Gnér., Icon. Régne Animal, Ins. p. 502 (1843) (Glaucopis). - Druce, Biol. Centr.-Am., Het. 1, p. 43 (1884).

niveifascia Walk., List Lep. Ins. Br. Mus. 7, p. 1628 (1856) (Calonota).

Wrighti Gundl., Ent. Cuba, Lep. p. 243 (1881) (Hippola).

ab. Angasi Druce, Proc. Zool. Soc. Lond. p. 321, t. 30, f. 5 (1884). - Hamps., Cat. Lop. Phal. I, p. 307 (1898).

ab. vidua Mén., Cat. Lép. Pétersb. 2, p. 141, t. 14, f. 6 (1857) (Glancopis). - Hamps., Cat. Lep. Phal. 1, p. 307 (1898).

ab. s piracula Mén., Cat. Iep. Pétersb. 2, p. 142 (1857) (Glaucopis). - Hamps., Cat, Lep. Phal. 1, p. 307 (1898).

vulcana Druce, Ann. \& Mag. Nat. Hist. (6) 4, p. 83

(1889); Biol. Centr.-Am., Hut. 2, p. 333, t. 70, f. 25 (1896). - Hamps., Cat. Lep. Phal. 1, p. 307 9 (1898).

\author{
Mexico, \\ Cuba, \\ Bahama-I., \\ Santa \\ Lucia, \\ Dominica, \\ Barbados, \\ Martinique
}

\section{Enope Walk.}

Walk., List Lep. Ins. Br. Mus. 1, p. 208 (1854). - Hamps., Cat. Lep. Phal. 1, p. 309 (1898).

Trichela Herr.-Schäff., Aussereur. Schmett. p. 78 (1853).

tolumnensis Herr.-Schäff., Aussercur. Sehmett. p. 73, Colombia f. 53 (1853) (Glaucopis). - Butl., Illustr: Het. Br. Mns. I, p. 24, t. 13, f. 10 (1877). - Hamps., Cat. Lep. Phal. I, p. 309, f. 140 (1898).

hirsuta Walk., List Lep. Ins. Br. Jius. 1, p. 208 I (1854) (Euchromia).

\section{Xanthomis Hamps.}

Hamps., Cat. Lep. Phal. 1, p. 309 (1898).

grandis Druce, Biol. Centr.-Am., Het. 1, p. 75, t. 8, f. 25 Costarica (1884) (Automolis). - Hamps., Cat. Lep. Phal. I, 1 p. 310 , f. 141 (1898).

Iistiaea Walk.

Walk., List Lep. Ins. Br. Mus. 1, p. 216 (1854). - Butl., Journ. Linn. Soc. Lond., Zool. I2, p. 36I, t. 28, f. 21 (I876). - Möschl., Verh. zool.-bot. Ges. Wien 27, p. 632 (1877). - Hamps., Cat. Lep. Phal. 1, p. 310 (1898). Sphenoptera Fied., Reise Nov., Lep. Atlas 1). 9 (1869) (nec Sol. 1833). 
amazoniea Butl., Journ. Linn. Soc. Lond., Zool. 12, p. 362 (1876); Illustr. Het. Br. Mus. 1, p. 21, t. 18, f. 1 (1877). - Hamps., Cat. Lop. Phal. 1, p. 314 (1898).

ab. inferioris Butl., Journ. Iinn. Soc. Lond., Zool. 12, p. 362 (1898). - Hamps., Cat. Lep. Phal. 1, p. 314 (1895).

bellatrix Walk., List Lep. Ins. Br. Mus. 1, p. 217 (1854) (Euchromia). - Butl., Iullstr. Het. Hr. Mus. 1, p. 21 , t. 8 , f. 8 (1877). - Hamps., Cat. Lep. Phal. 1, p. 312 (1898).

ab. Columbiae (Colombiae) Butl., Journ. Linn. Soc. Lond., Zool. 12, p. 362 (1876). - Hamps., Cat. Lep. Phal. 1, p. 312 (1898).

Biologic: Hahnel, Iris 3, p. 147 (1890).

boliviana Druce, Proc. Zool. Soc. I.ond. p. 493 (1890). - Hamps., Cat. Lep. Plial. 1, p. 313, t. 11, f. 10 (1898).

cepheus Cram., Pap. Exot. 3, t. 197, f. E (1779) (Sphinx). - Sepp, Snrin. Vlind. 1, p. 89, f. 41 (1848). - Hamps., Cat. Lop. Plial. 1, p. 313 (1898).

Biologie: Sepp, Surin. Vlind. 1, p. \$9, t. 41 (1848). glaneozona Druce, Ann. \& Mag. Nat. Hist. (7) 1, p. 405 (1898). - Hamps., Cat. Lep. Phal. 1, p. 315, t. 11 , f. 12 (1898).

Hoffmanusi Rothsch., Nov. Zool. 18, p. 39 (1911).

imaon Hamps., Cat. Lcp. Phal. 1, p. 311, t. 11, f. 13 (1898).

maon Druce, Ann. \& Mag. Nat. Hist. (6) 18, p. 29 (1896). - Hamps., Cat. Lep. Phal. 1, p. 313, t. 11, f. 11 (1898).

Melilolae Butl., Journ. Linn. Soc. Lond., Zool. 12, p. 362 (1876). - Druce, Biol. Centr.-Am., Hct. 1, p. 42, t. 6, f. 14 (1884). - Waterh., Aid to Identif. 1, t. 63 (1880-82). - Hamps., Cat. Lep. Plal. 1, p. 311 (1898).

bellatrix var. Walk., List Lep. Ins. Br. Mus. 1, p. 218 (1854) (Euchromin).

Biologic: Druce, Biol. Centr.-Am., Het. 1, p. 42 (1884).

monticola Klages, Proc. U. S. Nat. Mus. 29, p. 538 (1906). pauliua Walk., List Lep. Ins. Br. Mus. 35, p. 1808 (1866). - Hamps., Cat. Lep. Phal. 1, p. 310, f. 142 (1898).

phalerina (falerina) Druce, Ann. \& Mag. Nat. Hist. (7) 20 , p. $506(1907)$

proserpina Hübn., Zutr. Exot. Sclimett. 2, p. 10, f. 221, 222 (1827) (Euchromia). - Feld., Tien. Ent. Monatssclir. 6, p. 53 (1862) (var.). - Preiss, Abbild. Nachtschin. p. 7, t. 9, f. 5 (1888). Hamps., Cat. Lep. Phal. 1, p. 314, f. 143 (1898). Biologic: Hahnel, Iris 3, p. 308 (1890).

tima Walk., List Lep. Ins. Br. Mus. 1, p. 233 (1854) (Euchromia). - Butl., Illustr. Het. Br. Mus. 1, p. 23 , t. 8, f. 9 (1877). - Hamps., Cat. Lep. Phal. 1, p. 315 (1898).

Batesi Feld., Reise Nov., Lep. 2, p. 9, t. 102, f. 35 14
Amazonas

Colombia, Venezuela

Bolivia

Trinidad,

Venezuela,

Surinain

Amazonas

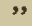

"

Bolivia

Panama, Venezuela,

Trinidad

Venezuela

São Paulo

Peru

Amazonas, Peru

Amazonas, Rio Grande do Sul 


\section{Agyrtidia Hamps.}

Hamps., Cat. Lep. Phal. 1, p. 315 (1898).

uranophila Walk., List Lep. Ins. Br. Mus. 35, p. 1874 (1866) (Eucyane). - Butl., Illustr. Het. Br. Mus. 1, p. 21, t. 18, f. 2 (1877). - Hamps., Cat. 1 Lep. Phal. 1, p. 316, f. 144 (1898).

Peru,

São Paulo

\section{Macrocneme Hübn.}

Hübn., Verz. bek. Schmett. p. 124 (1826). - Möschl., Verh. zool.-bot. Ges. Wien 27, p. 634 (1877). - Hamps., Cat. Lep. Phal. 1, p. 316 (1898).

Copaena Herr.-Schäff., Ausscreur. Schmett. 1, p. 22 (1854). Burm., Descr. Phys. Rep. Argent. 5, p. 387 (1878).

Biologie: Scitz, Stett. Ent. Zeitg. 5l, p. 261 (1890) (Mimicry). - Haase, Bibl. Zool. 8 (2), p. 76 (1893) (Mimicry).

adonis Druce, Biol. Centr.-Am., Het. 1, p. 48, t. 6, f. 16 (1884); 2, p. 337 (1897). - Hamps., Cat. Lep. Phal. 1, p. 318 (1898).

aeneus (eacus) Cram., Pap. Exot. 4, p. 129, t. 357, f. B (1781) (Sphinx). - Hamps., Cat. Lep. Phal. 1, p. $326(1898)$.

errans Hübn., Vorz. bck. Schmett. p. 124 (1826) (Pseudomya).

pusilla Butl., Journ. Iinn. Soc. Lond., Zool. 12, p. 372 (1870) (Mastigocera).

affinis Klages, Proc. U. S. Nat. Mus. 29, p. 539 (1906). albitarsia Hamps., Cat. Lep. Phal. 1, p. 325, t. 12, f. 6 (1898).

alesa Druce, Proc. Zool. Soc. Lond. p. 493 (1890). Hamps., Cat. Lep. Phal. 1, p. 325 , t. 12, f. 3 (1898).

auripes Walk., List Lep. Ins. Br. Mus. 1, p. 250 (1854) (Euchromia). - Butl., Illustr. Het. Br. Mus. 1, p. 25, t. 8, f. 4 (1877). - Druce, Biol. Centr.-Am., Het. 1, p. 47, t. 6, f. 15 (1884). - Hamps., Cat. Lep. Phal. 1, p. 323 (1898).

caerulcscens Dogn., Ann. Soc. Ent. Belg. 50, p. 180 (1906). caurensis Klages, Proc. U. S. Nat. Mus. 29, p. 540 (1906). chrysitis Gnér., Icon. Règne Animal, Ins. p. 502 (1843) (Glaucopis). - Druco, Biol. Centr.-Am., Het. 2, p. 337 (1897). - Hamps., Cat. Lep. Phal. 1, p. 319 (1898).

iole Druce, Biol. Centr.-Am., Het. 1, p. 48, t. 6 , f. 17 (1884); 2, p. 337 (1897).

clirysotarsia Hamps., Cat. Lep. Phal. 1, p. 324, t. 12 , f. 22 (1898). - Klages, Proc. U. S. Nat. Mus. 29, p. 540 (1906).

eluyras Schaus, Ent. Am. 5, p. 88 (1889). - Druce, Biol. Centr.-Am., Het. 2, p. 337, t. 71, f. 4 (1897).

- Hamps., Cat. Lep. Phal. 1, p. 319 (1898).

cupreipennis Walk., List Lep. Ins. Br. Mus. 7, p. 1632 (1856). - Hamps., Cat. Lep. Phal. 1, p. 318, t. 11 , f. 15 (1898).

Mexico, Guatema a, Panama

Surinam, Pará

Venezuela

Amazonas

Peru,

Bolivia

Honduras, Guatemala, Costarica, Panama, Colombia

Venezucla

Mexico bis

Panama,

Surinam, Rio Grande do Sul

Panama (I. Taboga),

Venezucla

Mexico

Brasilien (ubi?, M. C. Vind.) 
eyanea Butl., Journ. Linn. Soc. Lond., Zool. 12, p. 372 (1876) (Mastigocera). - Hamps., Cat. Lep. Phal. 1, p. 317 , t. 11, f. 14 (1898).

esmeralda Butl., Journ. Linn. Soc. Lond., Zool. 12 , p. 371 (1876). - Hamps., Cat. Lep. Phal. I, p. 325 , t. 12 , f. 4 (1898).

evelina Druce, Biol. Centr.-Am., Het. 1, p. 48, t. 8, f. 4 (1884); 2, p. 337 (1897). - Hamps., Cat. Lep. Phal. 1, p. 320 (IS98).

Biologie: Haasc, Bibl. Zool. (2) 8, p. 76 (1893) (Mimicry).

guyanensis Dogn., Het. nouv. Am. Sud 2, p. 6 (1911).

lesione Druce, Ann. \& Mag. Nat. Hist. (6) 2, p. 239 (1888); Biol. Centr.-Am., Het. 2, p. 337, t. 71, f. 3 (1897). - Hamps., Cat. Lep. Phal. 1, p. 326 (1898).

imman is Hamps., Cat. Lcp. Phal. 1, p. 320 , t. 12, f. 1 (1898).

- indistineta Butl., Journ. Linn. Soc. Lond., Zool. 12 , p. 371 (1876). - Hamps., Cat. Lep. Phal. 1, p. 324 , f. 148 (1898).

splendida Butl., Journ. Linn. Soc., Zool. 12, p. 371 (1876).

cyllarus Druce, Ann. \& Mag. Nat. Hist. (6) 18, p. 29 (1896); Biol. Centr.-Am., Het. 2, p. 336, t. 71, f. 2 (1896).

ab. Hampsoni Schrottky, Iris 24, p. 151 (1910). indistincta ab. I Hamps., Cat. Lep. Phal. 1, p. 324 (1898).

jalapensis Schaus, Ent. Am. 5, p. 89 (1889) (Callicarus). - Druce, Biol. Centr.-Am., Het. 2, p. 338 , t. 71 , f. 7 (1897). - Hamps., Cat. Lep. Phal. 1, p. 323 (1898).

laciades Schaus, Ent. Am. 5, p. 88 (1889) (Callicarus). - Druce, Biol. Centr.-Am., Het. 2, p. 338 (1597). - Hamps., Cat. Lep. Phal. 1, p. 323, t. 12 , f. 2 (1898).

laconia Druce, Fiol. Centr.-Am., Het. 1, p. 49, t. 6, f. 18 (1881); 2, p. 338 (1897) (Callicurus). - Hamps., Cat. Lep. Phal. 1, p. 319, f. 146 (J898).

- lades Cram., Pap. Exot. 1, p. 131, t. 83, f. E (1776) (Sphinx). - Hamps., Cat. Lop. Phal. 1, p. 317, f. 145 (1898).

leucostigma Perty, Delect. Anim. Artic. p. 158, t. 31, f. 11 (1834) (Glaucopis). - Druce, Biol. Centr.-Am., Het. 1, p. 47 (1881); 2, p. 336 (1896). naja Burm., Descr. Phys. Rep. Argent. 5, p. 387 (1878) (Copaena) (nec Z.).

ab. ferrea Butl., Journ. Linn. Soc. Lond., Zool. 12, p. 371 (1876). - Drucc, Biol. Centr.-Am., Hot. 2, p. 337 (1897). - Hamps., Cat. Lep. Phal. 1, p. 318 (1898).

Biologie: Schrottky, Iris 22, p. 128-130 (1909) (Mimicry).
Rio Janeiro

Panama (I.

Taboga),

Amazonas,

Mexico, Panama

Franz.-

Guayana

Panama

Bolivia

Panama, Colombia,

Amazonas

(M.C.Vind.),

Pará,

Rio Janeiro

(M.C.Vind.),

Santa

Catharina

(M.C.Vind.)

Rio Grande

do Sul,

Paraguay

Mexico

,

Mexico, Guatemala

Mexico bis Pcru u.

Argentinien 
uaju Fabr, Mant. Ins. 2, p. 106 (1787) (Zygaena). Hïbn., Zutr. Exot. Selnmett. 1, p. 15, f. 65, 66 (1827). - Feld., Wien. Ent. Monatsschr. 6, p. 54 (1862). - Hamps., Cat. Lep. Phal. 1, p. 322 , f. 147 (1898).

maroniensis Solıaus, Proe. U. S. Nat. Mus. 29, p. 188 (1905).

misitra Seliaus, Ent. Am. 5, p. 88 (1889) (Callicarus). - Druce, Biol. Centr.-Am., Het. 2, p. 338 t. 71, f. 6 (1897). - Hanps., Cat. Lep. Phal. 1, p. 323 (1898).

nigritarsia Hamps., Cat. Lep. Plıal. 1, p. 326, t. 12, f. 24 (1898).

vittata Druce, Biol. Centr.-Am., Het. 1, p. 48 (1884); 2, p. 336 (1896) (nee Waik.).

nordlua Schaus, Journ. New York Ent. Soc. 9, p. 41 (1901).

semiviridis Druce, Ann. \& Mag. Nat. Hist. (8) 7, p. 287 (1910).

sura Schaus, Journ. New York Ent. Soc. 9, p. 42 (1901). thyra Möschl., Verh. zool.-bot. Ges. Wien 32, p. 334, t. 18, f. 24 (1883). - Hamps., Cat. Lep. Phal. 1, p. 321 (1898).

thyridia Hamps., Cat. Lep. Phal. 1, p. 321 , t. 11, f. 9 (1898). - Klages, Proc. U. S. Nat. Mus. 29, p. 539 (1906).

viridifusa Schaus, Trans. Am. Ent. Soc. p. 135 (1904).

vittata Walk., List Lep. Ins. Br. Mus. 1, p. 249 (1854) (Euchromia). - Hamps., Cat. Iep. Phal. 1, 34 p. 325 , t. 12 , f. 23 (1898).
Surinam, Amazonas, Pará

Franz.-

Guayana

Mexico

Mexico,

Brit.-

Honduras,

Honduras,

Guatemala,

Trinidad

Mexico

Colombia

Rio Janeiro

Trinidad, Surinam, Pará, Colombia,

Peru, Bolivia

Venezuela, Surinam

Paraná

Pará,

Venezuela

\section{Phaeo Neum.}

(Phaio) Neum., Can. Ent. 26, p. 334 (1894). - Hamps., Ann. \& Mag. Nat. Hist. (7) 15, p. 428 (1905).

Mydropastea Hamps., Cat. Lep. Phal. 1, p. 326 (1898).

aequigutfata (acquiguttata) Dogn., Ann. Soe. Ent. Belg. Colombia p. $216(1909)$.

albicheta Schaus, Journ. New York Ent. Soc. 4, p. 131 (1896) (Eupyra). - Hamps., Cat. Lep. Phal. 1, p. 328 , f. 150 (1898).

aurata Schaus, Proc. Zool. Soc. Lond. p. 273 (1892) (Eupyra); Am. Lep. p. 2, t. 1, f. S (1892). Hamps., Cat. Lep. Phal. 1, p. 329 (1898).

bacelıans Schaus, Proc. Zool. Soc. Lond. p. 274 (1892) (Eupyra); Am. Lep. p. 3, t. 1, f. 5 (1892). Hamps., Cat. Lep. Phal. 1, p. 329 (1898).

eaeruleonigra Schaus, Proo. U. S. Nat. MLus. 29, p. 188 (1905).

cephalena Druce, Proc. Zool. Soc. Lond. p. 372, t. 39 , f. 2 (1883) (Eupyra). - Hamps., Cat. Lep. Phal, 1, p. 327, f. 149 (1898).

Ecuador

Peru

Colombia, Ecuador 
geminiguttata Dogn., Hét. nouv. Am. Sud. 2, p. 7 (1911). longipenu is Neum., Can. Ent. 26, p. 335 (1891).

piruclı Dogn., Ann. Soe. Ent. Belg. 41, p. 22 (1897) (Eupyra) (huius generis?).

quadriguttafa Dogn., Ann. Soc. Ent. Bolg. 53, p. 216 (1909).

Salmoni Druee, Proc. Zool. Soc. Lond. p. 372 (1883) (Eupyra). - Hamps., Cat. Lep. Phal. I, p. 328, t. 12, f. 13 (1898).

silva (sylva) Sehaus, Journ. New York Fint. Soc. 4, p. 131 (1896) (Eupyra). - Hamps., Cat. Iep. Phal. 1, p. 328 , t. 12 , f. 11 (1898).

unimacula Rothseh., Nov. Zool. 18, p. 39 (1911).

Colombia

Cuba

Eeuador

,

Colombia

Rio Janeiro 13

\section{Chrysocale Walk.}

Walk., List Lep. Ins. Br. Mus. 1, p. 208 (1854). - Hamps., Cat. Lep. Phal. 1, p. 329 (1898).

corax Hamps., Ann. \& Mag. Nat. Hist. (7) 8, p. 171 (1901).

iercns Schaus, Journ. New York Ent. Soc. 4, p. 131 (1896) (Eupyra). - Hamps., Cat. Lep. Phal. 1, p. 332 , t. 12, f. 21 (1898).

gigantea Druee, Proc. Zool. Soc. Lond. p. 493 (1890) (Eupyra). - Hamps., Cat. Lop. Phal. 1, p. 331, t. 12 , f. 16 (1898).

gigas Rothsch., Nor. Zool. 18, p. 39 (1911).

ignita Herr.-Sehäff., Aussercur. Schmett. p. 73, f. 55 (1853) (Glaucopis). - Butl., Illustr. Het. Br. Mus. 1, p. 23, t. 10, f. 8 (1877). - Hamps., Cat. Iep. Phal. 1, p. 332, f. 152 (1898).

pleheja Herr.-Schäff., Aussercur. Sehmett. p. 73, f. 56 (1883) (Glaucopis). - Butl., Illustr. Het. Br. Mus. 1, p. 24, t. 10, f. 5 (1877). - Hamps., Cat. Lep. Phal. 1, p. 331 (1898).

opulenta Walk., List Lep. Ins. Br. Mus. 1, p. 210 (1851) (Euchromia).

prineIpalis Walk., List Lep. Ins. Br. Mus. 3I, p. 97 (1864) (É upyra). - Druee, Biol. Centr.-Am., Het. 1, p. 43 (1884); 2, p. 333, t. 70 , f. 27 (1896). - Hamps., Cat. Iep. Phal. 1, p. 330, f. 151 (1898).

quadruplex Dogn., Ann. Soc. Ent. Belg. 50, p. 180 (1906). rogalis Boisd., Spéc. Gén. Lép. I, t. 16, f. 3 (1836) (Glaucopis). - Herr.-Schäff., Aussereur. Sehmett. p. 73, f. 57 (1853). - Burm., Descr. Phys. Rep. Argent. 5, p. 386 (1878). - Hamps., Cat. Lep. Phal. 1, p. 330 (1898).

ab. quadripunctata Dogn., Ann. Soc. Ent. Belg. 41, p. 23 (1897) (Eupyra).

splendens Dogn., Le Nat. (2) 2, p. 152 (1888) (Eupyra); Lép. Loja p. 47, t. 4, f. I (1891). - Hamps., Cat. Lep. Phal. 1, p. 331 (1898).

10

Peru

Bolivia,

Peru

Colombia

Colombia, Eeuador, Peru

Venezuela, Colombia, Brasilien (ubi ?) (M. C. Vind.)

Venezuela

Mexico,

Guatemala

Colombia

Eeuador,

Argen-

tinien

Eenador

, 


\section{Calonotus Hübn.}

(Calonotos) Hübn., Vcrz. bek. Schmett. p. 123 (1826). - Möschl., Verh. zool.-bot. Ges. Wien 27, p. 633 (1877). — Hamps., Cat. Lep. Phal. 1, p. 332 (1898). antennatus(-a) Rothsch., Nov. Zool. 18, p. 39 (1911).
auratus(-a) Walk., List Lep. Ins. Br. Mus. 1, p. 250 (1854) (Euchromia). - Hamps., Cat. Lep. Phal. 1, p. 335 , f. 155 (1898).

ehaleipleurus(-a) Hamps., Cat. Lep. Phal. 1, p. 334, t. 12 , f. 8 (1898).

ehryseis Druee, Ann. \& Mag. Nat. Hist. (7) 1, p. 401 (1898). - Hamps., Cat. Lep. Phal. 1, p. 336, t. 12 , f. 28 (1898).

helymus Cram., Pap. Exot. 1, p. 4, t. 2, f. D, E (1775) $(\operatorname{Sphin} x)$. - Sepp., Surin. Vlind. 2, p. 125, t. 59 (1848). - Hamps., Cat. Lep. Phal. 1, p. 335 (1898).

aterrima Sepp, Surin. Vlind. 2, p. 217, t. 97 (1848) (Glancopis).

Biologie: Sepp, Surin. Vlind. 2, p. 125, t. 59; p. 217 , t. 97 (1848).

Hoffmannsi Rothsch., Nov. Zool. 18, p. 40 (1911).

longipennis Rothsch., Nov. Zool. 18, p. 40 (1911).

metalliens Druce, Biol. Centr.-Am., Het. 1 , p. 45 , t. 8 , f. 2 (1884). - Hamps., Cat. Lep. Phal. 1, p. 333, f. $153(1898)$.

opalizans Rothseh., Nor. Zool. 18, p. 40 (1911).

phlegmon Gram., Pap. Exot. 1, t. 35, f. H (1775) (Sphinx). - Hamps., Cat. Lep. Phal. 1, p. 334 (1898).

plumulatus Klages, Proe. U. S. Nat. Mus. 29, p. 541 (1906). tiburtus Cram., Pap. Exot. 3, p. 75, t. 237, f. C (1780) (Sphinx). - Druce, Biol. Contr.-Am., Het. 1, p. 44 (1884). - Hamps., Cat. Lep. Phal. 1, p. 333 , f. 154 (1898).

triplagus (-n) Hamps., Ann. \& Mag. Nat. Hist. (8) 4, p. $346(1909)$.

tripunctatus Hamps., Cat. Lep. Phal. 1, p. 335 t. 12 , f. 7 (1898) (nec Druce).

tripunetatus (-n) Druce, Ann. \& Mag. Nat. Hist. (7) 1, 14 p. 401 (1898).

Venczucla

,"

,

Colombia, Bolivia

Surinam

Amazonas

Surinam

Costariea, Panama

Venezuela

Surinam, Pará

Venczuela

Costarica, Panama, Venezuela, Surinam Amazonas

St. Vineent, Trinidad

\section{Poliopastea Hamps:}

Hamps., Cat. Lep. Phal. 1, p. 336 (1898).

obscura Wallengr., Wien. Ent. Monatsschr. 4, p. 40 (1860) (Tipulodes). - Hamps., Cat. Lep. Phal. 1, p. 337, f. 157 (1898).

nox Druce, Ann. \& Mag. Nat. Hist. (7) 1, p. 208 (1898) (Chloropsinus).

Dekendeni Rothsch., Nov. Zool. 18, p. 40 (1911).

Eeuador

paro Dogn., Ann. Soc. Ent. Belg. 37, p. 368 (1893) (Thysanoprymna). - Hamps., Cat. Lep. Phal. 1 , p. 336 , f. 156 (1898).

Brit.-

Guayana,

Peru

Venezuela 
plumben Hamps., Cat. Lep. Phal. 1, p. 337, t. 12, f. 26

Trinidad, (1898)

Rosenbergi Rothsch., Nov. Zool. 18, p. 40 (1911). viridis Druec, Proc. Zool. Soc. Lond. p. 380 (1883) (Chloropsinus). - Hamps., Cat. Lep. Plial. 1, p. 337, t. 12 , f. 27 (1898).

viridivittata (verdivittata) Klages, Proc. U. S. Nat. Venczuela Mus. 29, p. 541 (1906).

ab. fenestrata Klages, Proc. U. S. Nat. Mus. 29, 7 p. 542 (1906).

\section{Dinia Walk.}

Walk., List Lep. Ins. Br. Mus. 1, p. 189 (1854). - Hamps., Cat. Lep. Phal. 1, p. 338 (1911).

Ha ematerion Herr.-Schäff., Aussereur. Schmett. p. 22 (1854). Burm., Deser. Phys. Rep. Argeut. 5, p. 376 (1878).

La sio procta Wallengr., Ofvers. Sveuska Akad. Förh. 15, p. 135 (1858).

aeagrus (eagrus) Cram., Pap. Exot. 3, p. 10, t. 198, f. C (1779) (Sphinx). - Druce, Biol. Centr.-Am., Hct. 1, p. 63 (1884). - Hamps., Cat. Lep. Phal. 1, p. 338 , f. 158 (1898).

auge Walk., List Lep. Ins. Br. Mius. 1, p. 189 (1854) (Glaucopis) (nec. L.). - Burm., Descr. Phys. Rep. Argent. z. 5, p. 377 (1878).

Biologie: Meldola, Proc. Ent. Soc. Lond. p. XXIV, fig. (1883). - Seitz, Stett. Ent. Zeitg. 51, p. 263 (1890) (Mimicry).

mena Hübn., Samml. Exot. Schmett. 2 (1827) (Eunomia). - Hamps., Cat. Lep. Phal. 1, p. 339 (1898).

saucia Walk., List Lep. Ins. Br. Mus.' 1, p. 190 (1854) (Glaucopis).

subapicalis Walk., List Lep. Ins. Br. Mus. 1, p. 190 (1854) (Glaucopis). - Hamps., Cat. Lep. Phal. 1, p. 339 , t. 12 , f. 12 (1898).

merra Wallengr., Wien. Ent. Monatsschr. 4, p. 41 (1860) (Lasioprocta).

3

Mesolasia Hamps.

Hamps., Cat. Lep. Phal. 1, p. 339 (1898).

Folderi Rothsch., Nov. Zool. 18, p. 41 (1911).

haemorrhoidalis Stoll, Suppl. Cram. p. 53, t. 12, f. 1 (1790) (Sphinx). - Hübn., Samml. Exot. Schmett. 2, (1827). - Burm., Dascr. Phys. Rep.

Mexico bis Panama, Surinam, Rio Janeiro, São Paulo (M.C.Vind.), Santa Catharina (M. C. Vind.), Paraguay, Argentinicn Panama ( $\mathrm{M}$. C. Vind.), Colombia, Trinidad, Venezuela, Brasilien (ubi?)

Peru
Surinam, Brasilien (ubi ?)

Brit.-

Honduras, Ecuador, 7* 
Argent. 5, p. 376 (1878). - Druce, Biol. Centr.Am., Het. 1, p. 64 (1884). - Hamps., Cat. Lep. Phal. 1, p. 340 , f. 159 (1898).

haemorrhusa Hübn., Verz. bek. Schmett. p. 120 (1826) (Aelhria).

Hampsoni Dogn., Auu. Soc. Ent. Bclg. 46, p. 228 (1902). melanobasis Druce, Ann. \& Mag. Nat. Hist. (6) 20, p. 304 (1897) (Haematerion). - Hamps., Cat. Lep. Phal. 1, p. 340 , t. 13, f. 8 (1898).

Brit.-Guayana (M. C. Vind.), Surinam, RioJanciro

Venezuela

Santa

Catharina,

Paraná

? ornata Mén., Cat. Lep. Pétersb. 2, p. 139, t. 14, f. 2 (1857) (Laemocharis). - Hamps., Cat. Lep. Phal. 1, p. 341 (1898) huius goneris?).

paula Sehaus, Proc. Zool. Soe. Lond. p. 227 (1894) (Aethria). - Hamps., Cat. Lop. Phal. 1, p. 340 , 6 t. 13, f. 15 (189s).

Minas Geraës

São Paulo

\section{Trichura Hübn.}

Hübn., Verz. bek. Schinctt. p. 126 (1826). - Hamps., Cat. Lep. Phal. 1, p. 341 (1898).

Cercophora Herr.-Schüff., Aussercur. Schmett. 1, p. 22 (1854).

Biologie: Seitz, Stett. Ent. Zeitg. 51, p. 263 (1890) (Mimicry). - Haasc, Bibl. Zool. 8 (2), p. 77 (1893) (Mimicry).

aurifera Butl., Journ. Iinn. Soc. Lond., Zool. 12, p. 405 (1876). - Hamps., Cat. Lep. Phal. 1, p. 345, t. 12 , f. 18 (1898).

melas var. Walk., Iist Lep. Ins. Br. Mus. 1, p. 194 (1854).

cerberus Pall., Spic. Zool. 9, p. 27, t. 2, f. 8 (1772) (Sphinx). - Hamps., Cat. Lep. Phal. 1, p. 342, f. 160 (1898).

caudata Fabr., Gen. Ins. p. 277 (1777) (Zygaena). urophora Horr.-Sehäff., Aussercur. Selimett. 2, p. 74, f. 266 (1855) (Cercophona).

Biologie: Haase, Bibl. Zool. 8 (2), p. 77 (1893) (Mimicry).

coaretata Drury, Ill. Exot. Ent. 2, p. 46, t. 27, f. 2 (1773) (Sphinx). - Hamps., Cat. Lep. Phal. 1, p. 344, f. 161 (1898).

Biologie: Haase, Bibl. Zool. \& (2), p. 77 (1893) (Mimicry).

eyanea Sehaus, Proc. Zool. Soc. Iond. p. 276 (1872). Hamps., Cat. Icp. Phal. 1, p. 345, t. 12, f. 25 (1898).

dixanthia Hamps., Cat. Lep. Phal. 1, p. 315 , t. 17, f. 18 (1898).

- Druryi Hübn., Verz. bek. Schmett. p. 126 (1826). Druce, Biol. Centr.-Am., Het. 1, p. 65 (1881); 2, p. 349 (1897). - Hamps., Cat. Lep. Phal. 1, p. 344 (1898).

coarctata Cram., Pap. Exot. 1, p. 7, t. 4, f. F, G (1775) (Sphinx) (nee Drury).

Venczucla, Surinam, Pará

Venezuela (AT. C. Vind.), Trinidad, Surinam, Ob. Amazouas (M. C. Vind.), Rio Janeiro: São Paulo

Venezucla, Pará,

Pernam-

buco, Rio Janeiro (M.C.Vind.) Rio Janeiro

Minas Geraës

Mexieo, Brit.Honduras, Honduras, Guatemala, Panama, Brit.Guayana 
esmeralda Walk., List Lcp, Ins. Br. Mís. 1, p. 194 (1854) (Glaucopis). - Butl., Hlustr. Het. Br. Mus. 1, p. 39, t. 13, f. 11 (1877). - Druce, Biol. Centr.-Am., Het. 1, p. 65, t. 7, f. 4 (1884). Hamps., Cat. Lep. Phal. 1, p. 342 (1898).

fasciata Rothsch., Nov. Zool. 18, p. 41 (1911).

? frigida Burm., Descr. Puep. Argent. 5, p. 516 (1878); Atias t. 24, f. 6 (1879) (Eurota). - Hamps., Cat. Lep. Phal. 1, p. 316 (1848) (huius generis?). fulvicaudata Lathy, The Ent. 32 , p. 118 (1899). grandis Kaye, The Ent. 44, p. 144 (1911).

latifascia Walk., List Lep. Ins. Br. Mus. 1, p. 193 (1854) (Glaucopis). - Butl., Mlustr. Het. Br. Mus. 1, p. 38, t. 11, f. 7 (1877). - Hamps., Cat. Lep. Plial. 1, p. 343 (1898).

v. ismene Mösch1., Verl. zool.-bot. Ges. Wien 27 , p. 639, t. 8, f. 9 (1877). - Hamps., Cat. Lep. Phat. 1, p. 343 (1898).

Ribbei Druce, Biol. Centr.-Am., Het. 1, p. 65, t. 8 , f. 15 (1884).

mathina Druce, Ann. \& Nag. Nat. Hist. (7) 1, p. 402 (1898). - Hamps., Cat. Lep. Phal. 1, p. 345, t. 12 , f. 17 (1898).

melanosoma Hamps., Cat. Lep. Phal. 1, p. 342, t. 12 , f. 5 (1898).

monstrabilis Klages, Pıoc. U. S. Nat. Mus. 29, p. 542 (1906).

pusilla Rothseh., Nov. Zool. 18, p. 157 (1911). 16
Brit.-

Honduras,

Honduras,

Guatemala, Colombia

Peru

Argentinien

\section{Paraguay}

São Paulo

Pará,

Rio Janciro

(MI.C. Vind.)

Panama, Colombia,

Surinam

Venezuela, Pará

Santa Catharina

Venezucla

Peru

\section{Corematura Butl.}

Butl., Journ. Linn. Soc. Lond., Zool. 12, p. 403 (1876). - Hamps., Cat. Lep. Phal. 1, p. 346 (1898).

aliaria Druce, Proe. Zool. Soe. Lond. p. 494, t. 42, f. 3 (1890) (Trichura). - Hamps., Cat. Lep. Phal. 1, p. 347, f. 163 (1898).

chrysogastra Perty, Delect. Anim. Artic. p. 157, t. 31, f. 10 (1834) (Glaucopis). - Hamps., Cat. Lep. Phal. 1, p. 346, f. 162 (1898).

postflava Guér., leon. Règne Animal, Ins. p. 501 (1843) (Glaucopis).

abdominalis Walk., List Lep. Ins. Br. Mus. 7, 2 p. 1617 (1856) (Eunomia).

\section{Pezoptera Butl.}

(Pezaptera) Butl., Journ. Linn. Soc. Lond., Zool. 12, p. 404 (1876). Hamps., Cat. Lep. Pilal. 1, p. 347 (1898).

sordida Walk., List Lep. Ins. Br. Mus. 7, p. 1617 (1856) (Eunomia). - Hamps., Cat. Lep. Phal. 1, p. 348, f. 164 (1898).

divisa Walk., List Lep. Ins. Br. Mus. 31, p. 83 1

Amazonas, Pará (1864) (Pleia). 
Homoneuronia Dyar.

Dyar, Proe. U. S. Nat. Mus. 29, p. 174 (1905). modesta Sehaus, Proc. U. S. Nat. Mus. 29, p. 188 (1905). Franz.1 Guayana

\section{Aethria Hübn.}

Hübn., Verz. bck. Schmett. p. 120 (1826). - Hamps., Cat. Lep. Phal. 1, p. 348 (1898).

Scytale Feld., Reise Nov., Lep. Atlas p. 9 (1869) (nee Latr. 1802). Hyela Mösehl., Verh. zool.-bot. Ges. Wien 27, p. 637 (1877) (nee Walk.).

analis Schaus, Journ. New York Ent. Soe. 9, p. 42 (1901). andromacha Fabr., Syst. Ent. p. 830 (1775) (Zygaena). - Butl., Illustr. Het. Br. Mus. 1, p. 38, t. 13, f. 3 (1877). - Hamps., Cat. Lep. Phal. 1, p. 349, f. 165 (1898).

sanguiflua Hübn.-Gcy., Zutr. Exot. Schmett. 4, p. 25, f. 697, 698 (1832) (Eunomia).

finalis Walk., List Lep. Ins. Br. Mus. 1, p. 190 (1854) (Glaucopis).

pennata Möschl., Vcrh. zool.-bot. Ges. Wien 27, p. 638 , t. 8 , f. 8 (1877) (Eunomia).

aller Hamps., Ann. \& Mag. Nat. Hist. (7) 15, p. 428 (1905). carnicauda Hamps., Cat. Lop. Phal. 1, p. 349, t. 12, f. 9 (1898) (nec Butl.).

carnicauda Butl., Journ. Linn. Soc. Lond., Zool. 12, p. 400 (1876) (Eunomia).

sanguiflua Walk., List Lup. Ins. Br. Mus. 1, p. 187 (185t) (Glaucopis) (nee Hübn.).

daltha Drucc, Ann. \& Mag. Nat. Hist. (6) 15, p. 44 (1895) (Eunomia). - Hamps., Cat. Lep. Phal. 1, p. 352 , t. 12 , f. 14 (1898).

dorsilincata Hamps., Cat. Lep. Phal. 1, p. 351, t. 12, f. 19 (1898).

eburneifera Feld., Reise Nov., Lep. 2, p. 5, t. 102, f. 21

(1869) (Glaucopis). - Hamps., Cat. Lep. Phal. 1, p. 351 (1898).

elisa (eliza) Klages, Proe. U. S. Nat. Mus. 29, p. 543 (1906).

fulvieauda Butl., Journ. Linn. Soc. Lond., Zool. 12, p. 401 (1876) (Eunomia). - Hamps., Cat. Lop. Phal. 1, p. 350 , t. 12 , f. 10 (1898).

gracilis Mösehl., Verh. zool.-bot. Ges. Wien 27, p. 637, t. 8, f. 6 (1877) (Hyela). - Hamps., Cat. Lep. Phal. 1, p. 351 (1898).

innotata Sehaus, Trans. Am. Ent. Soe. 30, p. 135 (1904). Langleyi Klages, Proc. U. S. Nat. Mus. 29, p. 543 (1906). loucaspis Cram., Pap. Exot. 1, p. 83, t. 52, f. C, D (1775) (Sphinx). - Mösehl., Verh. zool.-bot. Ges. Wien 27, p. 638 (1877). - Hamps., Cat. Lep. Phal. 1, p. 352, f. 166 (1898).

Peru

Venezuela, Surinam, Pará

Venezuela

Trinidad, Venezuela, Pará,

Santa Catharina (M.C.Vind.)

Pará

Jamaica

Amazonas

Venezuela

São Paulo

Surinam, Rio Janeiro

Rio Janeiro

Venezuela

Venezuela, Surinam, Brit.- \&

Franz.-

Guayana, Brasilien (ubi?), Bolivia 
ocina Druoc, Proc. Zool. Soc. Lond. p. 379, t. 39, f. 11 (1883) (Eunomia). - Hamps., Cat. Iep. Phal. 1, p. 353 (1898).

platyzona Feld., Roise Nov., Lep. 2, p. 9, t. 102, f. 24 (1569) (Scytale). - Hamps., Cat. Lep. Phal. 1, p. 353 (1898).

rubripectus (rubipectus) Schaus, Journ. New York Ent. Soc. 6, p. 139 (1898). — Hamps., Cat. Lep. Phal. 1, p. 353, t. 12, f. 20 (1898).

sanetula Dogn., Hét, nour. Am. Sud 2, p. 7 (1911).

sareosoma Butl., Journ. Linn. Soc. Lond., Zool. 12 , p. 401 (1876) (Eunomia). - Hamps., Cat. Lep. Phal. 1, p. 348 (1898).

laudamia Druce, Proc. Zool. Soc. Lond. p. 494, t. 42 , f. 1 (1890) (Dinia).

stipata Walk., List Lep. Ins. Br. Mus. 1, p. 184 (1854) (Glaucopis). - Butl., Illustr. Het. Br. Mus. 1, p. 38, t. 13, f. 8 (1877). - Hamps., Cat. Lep. 18 Phal. 1, p. 350 (1898).

\section{Paraethria Hamps.}

Hamps., Cat. Lep. Phal. 1, p. 353 (1898).

angustipenuis Rothsch., Nov. Zool. 18, p. 41 (1911).

flarosignata Rothsch., Nov. Zool. 18, p. 41 (1911).

triseriata Herr.-Schäff., Ausserour. Schmett. p. 74,

f. 261 (1855) (Gnophaela). - Hamps., Cat. Lcp. Phal. 1, p. 354, f. 167 (1898).

Biologie: Schrottky, Iris 24, p. 151 (1910).

3

\section{Aethriopsis Schrottky.}

Schrottky, Iris 24, p. 151 (1910).

barbata Schrottky, lris 24, p. 151 (1910).

1

Argyroides Butl.

(Argyroeides) Butl., Journ. Linn. Soc. Lond., Zool. 12, p. 403 (1876). Hamps., Cat. Lcp. Phal. 1, p. 354 (1898).

affinis Rothsch., Nov. Zool. 18, p. 41 (1911).

augiades Druce, Ann. \& Mag. Nat. Hist. (6) 18, p. 33 (1896) (Trichura). - Hamps., Cat. Lep. Phal. 1, p. 359 , t. 13 , f. 25 (1898).

auranticincta Klages, Proc. U. S. Nat. Mus. 29, p. 544 (1906).

boliviana Druce, Proc. Zool. Soc. Lond. p. 379, t. 39 , f. 12 (1883). - Hamps., Cat. Lep. Phal. 1, p. $356(1898)$.

braco Herr.-Schäff., Aussereur. Schmett. p. 74, f. 262 (1855) (Haematerion). - Hamps., Cat. Lep. Phal. 1, p. 356 (1898).

Biologie: Schrottky, Iris 22, p. 125, 128 (1909).
Bolivia

Colombia

Haiti

São Paulo

Colombia

Pará

Amazonass

Bolivia

Paraná,

Santa

Catharina,

Paraguay,

Argentinien

Paraguay
Minas Geraës

Colombia

(M.C.Vind.),

Bolivia

Venezuela

Bolivia

Venezucla, Sao Paulo, Paraguay 
ceres Drucc, Proc. Zool. Soc. Lond. p. 283 (1893) (Syntrichura ). - Hamps., Cat. Iep. Phal. 1, p. 357, t. 13 , f. 4 (1898).

eurypon Druce, Biol. Centr.-Am., Het. 1, p. 64, t. 7, f. 3 (1834); . 2, p. 349 (1897). - Hamps., Cat. Lep. Phal. 1, p. 358 (1898).

flavicineta Druce, Ann. \& Mag. Nat. Hist. (7) 15, p. 461 (1905).

flavieornis Rothsch., Nov. Zool. 18, p. 42 (1911).

flavipes Hamps., Cat. Lep. Phal. 1, p. 359, t. 13, f. 5 (1898).

Puscipes Rothsch., Nov. Zool. 18, p. 42 (1911).

liadassa Druce, Proc. Zool. Soc. Lond. p. 379 (1883) (T'richura). - Hamps., Cat. Lep. Phal. 1, p. 360, t. 13 , f. 29 (1898).

laurium (-on) Druce, Biol. Centr.-Am., Het. 1, p. 64, t. 8, f. 13 (1884). - Hamps., Cat. Lep. Phal. 1, p. 359, f. 170 (1898).

lydia Druce, Ann. \& Mag. Nat. Hist. (7) 11, p. 197 (1903).

Inagon Schaus, Proc. Zool. Soc. Lond. p. 276 (1892). Hamps., Cat. Lep. Phal. 1, p. 360, t. 13, £. 26 (1898).

gyas Druce, Ann. \& Mag. Nat. Hist. (6) 18, p. 32 (1896).

menephron Druce, Biol. Centr.-Am., Het. 1, p. 65, t. 8 , f. 14 (1884). - Hamps., Cat. Lep. Phal. 1, p. 355, f. 168 (1898).

Biologie: Haase, Bibl. Zool. (2) 8, p. 77 (1893) (Mimicry).

minuta Druce, Ann. \& Mag. Nat. Hist. (6) 2, p. 240 (1888); Biol. Centr.-Am., Het. 2, p. 349, t. 72, f. 3 (1897). - Hamps., Cat. Lep. Phal. 1, p. 358 (1898).

notha Schaus, Ann. \& Mag. Nat. Hist. (8) 7, p. 179 (1911).

ophion Walk., List Lep. Ins. Br. Mus. 1, p. 191 (1854) (Glaucopis). - Hamps., Cat. Lep. Phal. 1, p. 356, f. 169 (1898).

ortona Druce, Proc. Zool. Lond. p. 282 (1893). - Hamps., Cat. Lcp. Phal. 1, p. 355, t. 13, f. 3 (1898).

placida Druce, Ann. \& Mag. Nat. Hist. (6) 20, p. 304 (1897). - Hamps., Cat. Lep. Phal. 1, p. 357, t. 13 , f. 24 (1898).

quindiens is Dogn., Hét. nouv. Am. Sud 3, p. 4 (1911). rubricauda Dogn., Hét. nouv. Am. Sud 2, p. 8 (1911). sanguinea Schaus, Journ. Not York Ent. Soc. 4, p. 132 (1896). - Hamps., Cat. Lep. Phal. 1, p. 357, t. 13 , f. 23 (1898).

Biologie: Schrottky, Iris 22, p. 125, 128 (1909).

speetrum Schaus, Ann. \& Mag. Nat. Hist. (8) 7, p. 180 (1911).

strigula Druce, Ann. \& Mag. Nat. Hist. (6) 18, p. 32 (1896). - Hamps., Cat. Lep. Phal. 1, p. 354, t. 13 , f. 2 (1898).

Venezuela, Brit.-

Guayana

Guatemala,

Panama

Venezuela

Amazonas

Paraná

Minas Gerä̈s

Ecuador

Pauama

Rio Grande do Sul

Santa

Catharina

Panama

Honduras

Costarica

Honduras (M.

C. Vind.),

Vcnezuela,

Santa

Catharina

Ecuador

,

Colombia

Sĩo P’Paulo

(M.C.Vind.),

Paraná,

Rio Grande

do Sul,

Paraguay

Costarica

São Paulo 
suapurensis Klages, Proe. U. S. Nat. Mus. 29, p. 545 (1906).

?tricolor Pack., Rep. Peabody Acad. 1, p. 62 (1869) (Glaucopis). - Hamps., Cat. Lep. Phal. 1, p. 356 (1898) (huius gencris?).

variegata Kaye, The Ent. 44, p. 145 (1911).

vespina Schaus, Journ. Now York Ent. Soo. 9, p. 42 30

(1901). - Schrottky, Iris 24, p. 152 (1910).

Venezuela

Amazonas

Paraná

Paraná,

Paraguay

\section{Didasys Grote.}

Grote, Can. Ent. 7, p. 174 (1875). - Hamps., Cat. Lcp. Phal. 1, p. 360 (1898).

Belae Grote, Can. Ent. 7, p. 175 (1875). - Neum. \& Dyar, Florida Journ. New York Ent. Soe. 1, p. 103 (1893). - Hamps., Cat. Lep. Plial. 1, p. 360, f. 171 (1898). - Holl., Moth. Book p. 99, t. 13, f. 7, 8 1 (1903).

\section{Burtia Grote.}

Grote, Proc. Ent. Soc. Philarl. 6, p. 185 (Jul. 1866); Can. Ent. 33, p. 339 (1901); 34, p. 66 (1902). - Hamps., Ann. \& Mag. Nat. Hist. (7) 15, p. 429 (1905).

Gundlachia Herr.-Sehäff., Corresp.-Bl. min.-zool. Ver. Regensb. 20, p. 108 (Aug. 1860). - Hamps., Cat. Lep. Phal. 1, p. 361 (1898).

cruenta Herr.-Schäff., Corresp.-Bl. min.-zool. Ver. Regensb. 20, p. 108 (1866) (Gundlachia) (pr. p.). - Hamps., Cat. Lep. Phal. 1, p. 36l, f. 172 (1898).

rubella Grote, Proe. Ent. Soc. Philad. 6, p. 186, t. 5, f. 1 (1866). - Hamps., Cat. Lop. Phal. 1, p. 362, t. 13 , f. 9 (1898).

cruenta Herr.-Schäff,, Corresp.-Bl. min.-zool. Ver. 2 Regensb. 20, p. 108 (1866) (Gundlachia) (pr. p.).

\section{Hypocladia Hamps.}

Hamps., Cat. Lep. Phal. 1, p. 362 (1898).

alhipuncta Druce, Ann. \& Mag. Nat. Hist. (7) 15, p. 461 Peru (1905).

ealita Dogn., Hét. nouv. Am. Sud 2, p. 9 (1911).

elongata Druee, Ann. \& Mag. Nat. Hist. (7) 15, p. 461 (1905).

Cuba

militaris Butl., Illustr. Het. Br. Mus. 1, p. 33, t. 16, f. 1

Colombia

Venezuela p. 362 , f. 173 (1898).

parelpuneta Hamps., Ann. \& Mag. Nat. Hist. (8) 4, Brit. p. 347 (1909).

restricta Hamps., Ann. \& Mag. Nat. Hist. (7) 8, p. 172 Panama

Amazonas (1901). 


\section{Hyalomis Hamps.}

Hamps., Ann. \& Mag. Nat. Hist. (7) 15, p. 429 (1905).

Desmidocnemis Hamps., Cat. Lep. Phal. 1, p. 363 (1898) (nee Möschl.).

? espia Dogn., Ann. Soe. Ent. Belg. 41, p. 24 (1897) Eeuador (Desmidocnemis) (huius generis?).

hypochryseis Hamps., Cat. Lep. Phal. 1, p. 363, t. 13, São Paulo f. 27 (1898) (Desmidocnemis).

platyleuea Walk., List Lep. Ins. Br. Mus. 1, p. 198 Venezuela (1854) (Glaucopis). - Butl., Illustr. Het. Br. Mus. 1 , p. 35 , t. 13, f. 2 (1877). - Hamps., Cat. Lep. Plial. 1, p. 364, f. 174 (1898).

thyria Druce, Ann. \& MIag. Nat. Hist. (7) 1, p. 207 (1898) (Desmidocnemis). - Hamps., Cat. Lep. Phal. 1, 4 p. 363 , t. 13, f. $6(1898)$

Eeuador,

Bolivia

\section{Diptilum Prittw.}

(Diptilon) Prittw., Stett. Ent. Zeitg. 31, p. 349 (1870). - Hamps., Cat. Lep. Phal. 1, p. 364 (1898).

atereum(-a) Sehaus, Journ. Neฬw York Ent. Soe. 9, São Paulo p. 42 (1901).

aurantiipes Rothsch., Nov. Zool. 18, p. 42 (1911).

bivittatum(-a) Walk., List Lep. Ins. Br. Mus. 31, p. 90 (1864) (Cosmosoma). - Hamps., Cat. Lep. Phal. 1, p. 366, f. 177 (1898).

Biolog ie: Sclurottky, Iris 24, p. 152 (1910).

chrysocraspis Hamps., Cat. Lcp. Phal. 1, p. 365, t. 13, f. 7 (1898).

crassum Zerny, Iris 26 (1912) (ined.).

Docri Schans, Proc. Zool. Soc. Lond. p. 276 (1892) (Syntrichura); Am. Lep. p. 6, t. 1, f. 16 (1892).Hamps., Cat. Lep, Phal. 1, p. 365, f. 176 (1898). Biologic: Sohrottky, Iris 24, p. 152 (1910).

flavipalpe (-is) Hamps., Ann. \& Mag. Nat. Hist. (8) 8, p. 395 (1911).

halteratum (-a) Fabr., Syst. Ent. p. 555 (1775) (Zygaena). - Hamps., Cat. Lep. Plial. 1, p. 365, f. 175 (1898).

dieides Prittw., Stett. Ent. Zeitg. 31, p. 350 (1870). Biologie: Schrottky, Iris 22, p. 125,128 (1909).

Hoffmannsi Rothselı., Nov. Zool. 18, p. 42 (1911). philocles Druee, Ann. \& Mag. Nat. Hist. (6) 18, p. 34 (1896) (Syntrichura). - Druce, Biol. Centr.-Am., Hot. 2, p. 350, t. 72 , f. 9 (1897). - Hamps., Cat. Lep. Phal. I, p. 366 (1898).

proleueum(-n) Druce, Ann. \& Mag. Nat. Hist. (7) 15, p. $462(1905)$.

silpha (sylpha) Dogn., Ann. Soe. Ent. Belg. 46, p. 228 (1902).

telainonophorum Prittw., Stett. Ent. Zeitg. 31, p. 350, t. 2 , f. 2 (1870). - Hamps., Cat. Lep. Phal. I, p. 364 (1898).

Amazonas

Brasilicn

(ubi),

Paraguay

Bolivia

Colombia

Santa

Catharina,

Paraguay

Argentinien

Venezuela,

RioJanciro,

São Paulo,

Paraná,

Paraguay

Peru

Prunama

São Paulo

Venezuela

Eeuador

Minas Geraës, Rio Janeiro 


\section{Syntrichura Butl.}

Butl., Journ. Linn. Soc. Lond., Zool. 12, p. 405 (1876). - Hamps., Cat. Lep. Phal. 1, p. 366 (1898).

melaena Dogn., Ann. Soc. Ent. Belg. 51, p. 224 (1907).

placida Druce, Biol. Centr.-Am., Het. 1, p. 66, t. 7, f. 8 (1884). - Hamps., Cat. Lep. Phal. 1, p. 367, f. 178 (1898).

virens Butl., Journ. Linn. Soc. Lond., Zool. 12, p. 405 (1876). - Hamps., Cat. Lep. Phal. 1, p. 367 (1898).

v. reba Druce, Ann. \& Mag. Nat. Hist. (6) 18, p. 33 (1896); Biol. Centr.-Am., Het. 2, p. 350, t. 72 , f. 7 (1897). - Hamps., Cat. Lep. Phal. 1, 3 p. 367 (1898).

Peru

Guatemala

São Paulo

Panama,

Colombia, Venezuela

\section{Herea Walk.}

Walk., List Lep. Ins. Br. Mus. 1, p. 197 (1854). - Hamps., Cat. Lep. Phal. 1, p. 367 (1898).

Desmidoonemis Möschl., Stett. Ent. Zeitg. 33, p. 346 (1872).

metaxantha(-us) Walk., List Lep. Ins. Br. Mus. 1, p. 198

(1854) (Glaucopis). - Butl., Illustr. Het. Br.

Mus. 1, p. 39, t. 12, f. 7 (1877). - Hamps., Cat.

Lep. Phal. 1, p. 368, f. 179 (1898).

Prittwitzi Möschl., Stett. Ent. Zeitg. 33, p. 348 (1872)

(Desmidocnemis). - Hamps., Cat. Lep. Phal. 1,

p. 363 (1898); Ann. \& Mag. Nat. Hist. (7) 15, p. 429 (1905).

xanthogaster Druce, Ann. \& Mag. Nat. Hist. (7) 1,

p. 404 (1898). - Hamps., Cat. Lep. Phal. 1, p. 368 , t. 13 , f. 28 (1898).

ruliceps Walk., List Lep. Ins. Br. Mus. 1, p. 198 (1854)

(Glaucopis). - Butl., Illustr. Het. Br. Mus. 1,

Pará

p. 39, t. 13, f. 4 (1877). - Hamps., Cat. Lep.

Phal. 1, p. 368 (1898).

collaris Herr.-Schäff., Aussereur. Schmett. p. 74, f. 422 (1856) (Cercophora). - Möschl., Verh. 3 zool.-bot. Ges. Wien 27, p. 639 (1877).

\section{Cumenogaster Herr.-Schäff.}

Herr.-Schäff., Aussercur. Sehmett. 1, p. 22, 23 (1854). - Hamps., Cat. Lep. Phal. 1, p. 368 (1898).

affinis Rothsch., Nov. Zool. 18, p. 42 (1911).

eumenes Herr.-Schäff., Ausscreur. Schmett. 1, p. 74, f. 423 (1856). - Hamps., Cat. Lcp. Phal. 1, p. 370 (1898).

haemocera (haemacera) Hamps., Cat. Lep. Phal. 1, p. 369, t. 13 , f. 1 (1898).

Venezuela

Surinam, Brasilien (ubi?)

Venezuela,

Franz.-

Guayana,

Amazonas

(M.C.Vind.) 
nigricauda Dogn., Hét. nouv. Am. Sud 2, p. 8 (1911).

notabilis Walk., List Lep. Ins. Br. Mus. 31, p. 94 (1864)

Franz.-

Guayana (Pseudosphex). - Hamps., Cat. Lep. Phal. 1, p. 369 , f. 180 (1898).

v. caurensis Klages, Proc. U. S. Nat. Mus. 29 , p. $546(1906)$.

pseudopolybia Schrottky, Iris 22, p. 135 (1909).

Biologie: Selurottky, Iris 22, p. 135 (1909) (Mimicry).

psendospheeia Hamps., Cut. Lep. Phal. 1, p. 369, t. 13, Pará f. $22(1898)$.

7

Urolasia Hamps.

Hamps., Cat. Lep. Plial. 1, p. 370 (1898).

albipnneta Druce, Ann. \& Mag. Nat. Hist. (7) 15, p. 462 (1905).

broden Sehaus, Journ. New York Ent. Soc. 4, p. 132 (1896) (Syntrichura). - Hamps., Cat. Lep. Phal. 1, p. 370 , f. 181 (1898).

opalocineta Druee, Ann. \& Mag. Nat. Hist. (7) 1, p. 404 (1898). - Hamps., Cut. Lep. Phal. 1, p. 370, t. 12 , f. 15 (1898).

Pará

Venezuela

Paraguay 3

Sesiura Hamps.

Hamps., Cat. Lep. Phal. 1, p. 371 (1898).

smaragdina Walk., List Lep. Ins. Br. Mus. 31, p. 92 (1864) (Eunomia). - Mösehl., Verh. zool.-bot. Ges. Wien 27, p. 638 (1877). - Hainps., Cat.

Lep. Pinal. 1, p. 371, f. 182 (1898). saturatissima Walk., List Lep. Ins. Br. Mus. 31, 1 p. 93 (186:) (Aethria).

Venezuela

Trinidad

Franz.-

Guayana

Venezuela, Surinam, Franz."

Guayana, Amazonas

Metastatia Butl.

Butl., Cist. Ent. 2, p. 115 (1876). - Hamps., Cat. Lep. Phal. 1, p. 371 (1898).

azurea Lathy, The Ent. 32, p. 118 (1899). pyrrhorrhoea Hübn., Zutr. Exot. Schmett. 1, p. 12,
f. 43, 44 (1827) (Hyelosia). - Hamps., Cat. 2 Lep. Phal. 1, p. 372, f. 183 (1898).

Eeuador

Brit.-

Guayana,

Pará

\section{Abrochia Herr.-Schäff.}

Herr.-Schäff., Ausserour. Schmctt. p. 21 (1854). - Burm., Deser. Phys. Rep. Argent. 5, p. 371 (1878).

Chrysostola Herr.-Schäff., Aussereur. Sehmett. p. 22 (1854). Hamps., Cat. Lep. Phal. 1, p. 372 (1898). 
aequalis Walk., List Lep. Ins. Br. Mus. 31, p. 87 (1864) (Isanthrene). - Hamps., Cat. Lep. Phal. 1, p. 377 , t. 13 , f. 14 (1898).

atridorsata Hamps., Ann. \& Mag. Nat. Hist. (8) 4, p. 347 (1909) (Chrysostola).

nugusta Druce, Biol. Centr.-Am., Het. 1, p. 62, t. 7, f. 19 (1S84); 2, p. 347 (1897) (Dycladia). Hamps., Cat. I.op. Phal. 1, p. 374 (1898).

consobriua Walk., List Lop. Ins. Br. Mus. 7, p. 1619 (1856) (Pseudosphex). - Hamps., Cat. Lep. Phal. 1, p. 376, t. 13, f. 11 (1898).

cosmosomoides Rothsch., Nov. Zool. 18, p. 157 (1911) (Chrysostola).

discoplaga Schaus, Proe. U. S. Nat. Mus. 29, p. 189 (1905) (Chrysostola).

Iycladioides Hcyl., C. R. Soc. Ent. Belg. 34, p. XIV (1890) (Haematerion). - Hamps., Cat. I.cp. Phal. 1, p. 373 , t. 13 , f. 18 (1898).

Inveriu Druee, Ann. \& Mag. Nat. Hist. (6) 18, p. 32 (1896) (Argyroeides); Biol. Centr.-Am., Het. 2, p. 349, t. 72 , f. 5 (1897). - Hamps., Cat. Lep. Phal. 1, p. 373 (1898).

fulvisplies Druee, Ann. \& Mag. Nat. Hist. (7) 1, p. 404 (1898) (Chrysostola). - Hamps., Cat. Lop. Plial.1, p. 377 , t. 13 , f. 13 (1898).

humilis Herr.-Sohäff., Aussereur. Sohmett. p. 73, f. 264 (1854) (Chrysostola). - Hamps., Cat. Lep. Phal. 1, p. 374 (1898).

julumito Dogn., Ann. Soc. Ent. Eelg. 42, p. 344 (1898) (Chrysostola).

melina (mellina) Herr.-Schäff., Aussereur. Schmett. p. 74, f. 427 (1855) (Chrysostola). - Hamps., Cat. Lep. Phal. 1, p. 376 (1898).

mellita Sehaus, Ann. \& Mag. Nat. Hist. (8) 7, p. 180 (1911) (Chrysostola).

noza Druce, Ann. \& Mag. Nat. Hist. (6) 18, p. 32 (1896) (Argyroeides); Biol. Centr.-Am., Het. 2, p. 349, t. 72 , f. 4 (1897). - Hamps., Cat. Lop. Phal. 1, p. 378 (1898).

munda Walk., Iist Lep. Ins. Br. Mus. 7, p. 1605 (1856) (Isanthrene). - Hamps., Cat. Lep. Phal. 1, p. 376 , t. 13 , f. 16 (1898).

pelopia Druee, Ann. \& Mag. Nat. Hist. (6) 20, p. 303 (1897) (Dycladia). - Hanps., Cat. Lep. Phal. 1, p. 375 , t. 13 , f. 20 (1898).

postiea Walk, List Lep. Ins. Br. Nus. 1, p. 178 (1854) (Glaucopis). - Hamps., Cat. Iep. Phal. 1, p. 375 , t. 13 , f. 19 (1898).

sanguiceps Druee, Ann. \& Mag. Nat. Hist. (7) 1, p. 405 (1898) (Chrysostola). - Hamps., Cat. Lep. Phal.1, p. 735 , t. 13 , f. 12 (1898).

sanguitarsia Hamps., Cat. Lep. Phal. 1, p. 374, t. 13 , f. 10 (1898) (Chrysostola).

singnlaris Walk., Tist Lep. Ins. Br. Mus. 1, p. 182 (1854) (Glaucopis). - Hamps., Cat. Lep. P'lial. 1, p. 376, t. 13, f. 21 (1898).

tetrazona Hamps., Cat. Lep. Phal. 1, p. 375, t. 13 , f. 17 (1898) (Chrysostola).
Venezuela, Amazonas

Brit.-

Guayana

Mexieo, Guatemala

Pará

Venczuela

Venezuela

São Paulo

Panama

Franz.Guayana

Brasilien (ubi?)

Eeuador

Venezuela, Pará

Costariea

Panama

Pará

Panama, Bolivia

Pará

Franz. Guayana

Bolivia

Pará

Bolivia, Peru, Minas Geraës 
zethus Hübn., Zutr. Exot. Schmett. 1, p. 13, f. 49, 50 (1827) (Pseudosphex). - Burm., Descr. Phys. Rep. Argent. 5, p. 371 (1878). - Hamps., Cat. 32

Lep. Phal. 1, p. 377, f. 181 (1898).

\section{Psendargyroides Klages.}

(Pseudargyroeides) Klages, Proc. U. S. Nat. Mus. 29, p. 546 (1906). eaurensis Klages, Proc. U. S. Nat. Mus. 29, p. 547 (1906). Venezuela 1

\section{Ecdemus Herr.-Schäft.}

Herr.-Schäff., Aussereur. Schnctt. p. 22, 23 (1854). - Hamps., Cat. Lep. Phal. 1, p. 378 (1898).

hypoleucus Herr.-Schäff., Aussercur. Schmett. p. 74, f. 420 Amazonas, (1854). - Hamps., Cat. Lep. Phal. 1, p. 378, Pará f. 185 (1898).

obscuratus (-um) Schaus, Ann. \& Mag. Nat. Hist. (8) 7, Costarica 2 p. 180 (1911).

\section{Agunaix Hamps.}

Hamsp., Cat. Lep. Phal. 1, p. 378 (1898).

laerimans (lacrumans) Schaus, Journ. New York Ent. Peru, Soc. 6, p. 139 (1898). - Hamps., Cat. Lep. Bolivia 1 Phal. 1, p. 379, f. 186 (1898).

\section{Cercopimorpha Butl.}

Butl., Journ. Linn. Soc. Lond., Zool. 12, p. 424, t. 29, f. 16 (1876). - Hamps., Cat. Lep. Phal. 1, p. 379 (1898).

homopteridia Butl., Journ. Linn. Soc. Lond., Zool. 12, Pará p. 424 (1876). - Hamps., Cat. Lep. Phal. 1, p. 379 , f. 187 (1898).

pectinata Walk., List Lep. Ins. Br. Mus. 1, p. 254 (1854) (Euchromia) (neo Fabr.).

meterythra Hamps., Cat. Lep. Phal. 1, p. 380, t. 14, f. 1 Bolivia (1898).

tetragonia Hamps., Cat. Lep. Phal. 1, p. 379, t. 14, f. 21 Venezuela 3 (1898).

\section{Teucer Kirby.}

Kirby, Cat. Het. p. 165 (1892). - Hamps., Cat. Lep. Phal. 1, p. 380 (1898).

Telioneura Fcld., Reise Nov., Lep. Atlas p. 9, (1869) (nec Rond. 1868). - Butl., Journ. Linn. Soc. Lond. Zool. 12, p. 419, t. 29, f. $12(1876)$. 
albapcx Druce, Ann. \& Mag. Nat. Hist. (7) 1, p. $40 \%$ (1898). - Hamps., Cat. Lep. Phal. 1, p. 381, t. 14 , f. 22 (1898).

apicalis Rothsch., Nov. Zool. 18, p. 42 (1911).

brevipennis Butl., Illustr. Het. Br. Mus. 1, p. 45, t. 16, f. 9 (1877) (Telioneura). - Hamps., Cat. Lcp. Phal. 1, p. 381, f. 188 (1898).

brunneus(-a) Hamps., Ann. \& Mag. Nat. Hist. (7) 11, p. 341 (1903).

carmania Druce, Proc. Zool. Soc. Lond. p. 379 (1883) (Pezaptera). - Hamps., Cat. Lep. Phal. 1, p. 382 , f. 190 (1898).

distinctus(-a) Rothsch., Nov. Zool. 18, p. 43 (1911):

germanus(-a) Rothsch., Nov. Zool. 18, p. 43 (1911).

glaucopis Feld., Reiso Nov., Lep. 2, p. 9, t. 102, f. 31 (1869) (T'elioneura). - Hamps., Cat. Lep. Phal. 1, p. 3S1, f. 189 (1898).

lypophaeus(-a) Hamps., Ann. \& Mag. Nat. Hist. (7) 15, p. $429(1905)$.

rubrothorax Rothsch., Nov. Zool. 18, p. 43 (1911).

subplenus(-a) Walk., List Lep. Ins. Br. Mus. 1, p. 266 (1854) (Euchromia). - Hamps., Cat. Lep. Phal. 1, 11 p. 380 , t. 14, f. 2 (1898).

\section{Epanyeles Butl.}

Butl., Journ. Linn. Soc. Lond., Zool. 12, p. 425, t. 29, f. 18 (1876). Hamps., Cat. Lep. Phal. 1, p. 382 (1898).

imperialis Walk., List Lep. Ins. Br. Mus. 1, p. 241 (1854) (Euchromia). - Drucc, Biol. Centr..Am., Het. 1, p. 80 (1884). - Hamps., Cat. Lep. Phal. 1, 1 p. 382, f. 191 (1898).

Guatemala,

Venezuela,

Amazonas,

Pará

\section{Pterygopterus Butl.}

Butl., Journ. Linn. Soc. Lond., Zool. 12, p. 411, t. 29, f. 10 (1876). Hamps., Cat. Lep. Phal. 1, p. 383 (1898).

eaerulcus Hamps., Cat. Lep. Phal. 1, p. 384 (1898). clavipennis Druce, Biol. Centr.-Am., Het. 2, p. 351 , t. 72 , f. 11 (1897) (nco Butl.).

clavipennis Butl., Journ. Linn. Soe. Lond., Zool. 12, p. 411 (1876). - Hamps., Cat. Lep. Phal. 1, p. 384, f. 192 (1898).

flavicornis Kaye, The Ent. 44, p. 142 (1911).

leucomelas (leucomela) Walk., List Lep. Ins. Br. Mus. 7, p. 1636 (1856) (Automolis). - Druce, Biol. Centr.-Am., Het. 2, p. 351 (1897). - Hamps., Cat. Lcp. Phal. 1, p. 383 (1898).

superbus Druce, Biol. Centr.-Am., Het. 1, p. 67, t. 8 , f. 7 (1884).

Biologie: Haase, Bibl. Zool. 8 (2), p. 76 (1893)

Mexico

(Mimicry).

Espiritu

Santo

Brit.-

Guayana

Panama, Ob. Ama-

zonas,

Pará 
Episcepsis Butl.

Butl., Illustr. Het. Br. Mus. 1, p. 49 (1877). - Hamps., Cat. Lep. Phal. 1, p. 384 (1898).

Centronia Möschl., Verh. zool.-inot. Ges. Wien 27, p. 646 (1877) (nee Hübn.).

eapysea Sehaus., Ann. \& Mag. Nat. Hist. (8) 6, p. 190 (1910).

eapyscoides Dogn., Hét. nouv. Am. Sud 2, p. 10 (1911).

dacmonis (demonis) Drucc, Ann. \& Miag. Nat. Hist. (6) 18, p. 38 (1896) (Eucereon); Biol. Centr.-Am., Het. 2 , p. 364 , t. 73 , f. 15 (1897). - Hamps., Cat. Lep. Phal. 1, p. 390 (1898).

dodaba Dyar, Proc. U. S. Nat. Nus. 38, p. 230 (1910).

dominicensis Rotlisch., Nov. Zool. 18, p. 44 (1911).

endodasia Hamps., Cat. Lep. Phal. 1, p. 389, t. 14, f. 24 (1898).

Prances Dyar, Proc. U. S. Nat. Mus. 38, p. 230 (1910).

gnoma Butl., Illustr. Het. Br. Mus. 1, p. 44, t. 16, f. 2 (1877) (Heliura). - Hamps., Cat. Lep. Phal. 1, p. 387 (1898).

gnomoides Sehaus, Ann. \& Mag. Nat. Hist. (8) 6, p. 190 (1910).

Irampsoni Rothsch., Nov. Zool. 18, p. 43 (1911).

hypolener Hamps., Cat. Lop. Phal. 1, p. 388, t. 14, f. 4 (1898).

lamia Druce, Biol. Centr.-Am., Het. 1, p. 74 (1884) (Heliura) (pr. p.) (neo Butl.).

inornata Walk., List Lep. Ins. Br. Mus. 7, p. 1636 (1856) (Automolis). - Hamps., Cat. Lep. Phal. J, p. 388, t. 14, f. 23 (1898).

lamia Druce, Biol. Centr.-Am., Het. 1, p. 74(1884); 2, p. 354 (1897) (Heliura) (pr. p.) (nee Butl.).

Klagesi Rothsch., Nov. zuol. 18, p. 44 (1911).

lamia Butl., Hllustr. Het. Br. Mus. 1, p. 44, t. 16, f. 3 (1877) (Heliura). - Hamps., Cat. Lep. Phal. 1, p. 387 , f. 195 (189S).

lenaeus Cram., Pap. Exot. 3, p. 95, t. 248, f. G (1780) (Sphin $x$ ). - Möschl, Verh. zool.-bot. Ges. Wien 27, p. 642 (1877). - Druce, Biol. Centr.-Am., Het. 1, p. 74 (1881) (pr. p.); 2, p. 354 (1897). Hamps., Cat. Lep. Phal. $1_{2}$ p. 385 (1898).

Bi ol ogie: Stoll, Suppl. Cram. p. 68 , t. 15, f. 4-6 (1790).

litoralis Rothseh., Nov. Zool. 18, p. 44 (1911).

melanitis Hübn., Zutr. Exot. Sehmett. 1, p. 19, f. 93, 94 (1827) (Centronia). - Hamps., Cat. Lcp. Phal. 1, p. 385 , f. 193 (1898).

melanota Hamps., Ann. \& Mag. Nat. Hist. (8) 4, p. 347 (1909).

Moloneyi Druce, Biol. Centr.-Am., Het. 2, p. 352, t. 72 , f. 16 (1897) (Aclytia). - Hamps., Cat. Lep. Phal. I, p. 389, f. 197 (I898).

obsoleta Burm., Deser. Phys. Rep. Argent. 5, p. 516 (1878) (Charidea). - Hamps., Cat. Lep. Phal. I, p. 389, f. 196 (1898).

Costariea

Franz.-

Guayana

Panama

Mexico, Venezucla

Dominiea

Paraná

Mexico

Amazonas, Rio Janeiro

Costariea

Surinam

Costariea

Mexieo, Guatemala, Costariea, Panama

Venezuela

Amazonas

Mexico, Costariea, Brit.Guayana, Surinam

Venezuela

Brit.Guayana, Surinam

Brit.-

Guayana

Brit.-

Honduras

Argentinien 
Ockendeni Rothisch., Nov. Zool. 18, p. 44 (1911). redunda Schaus, Am. \& Mag. Nat. Hist. (8) 6, p. 190 (1910).

rlypoperas (rypoperas) Hamps., Cat. Lep. Phal. 1, p. 386, t. $14, \mathrm{f} .3$ (1898).

lenaeus Druce, Biol. Centr.-Am., Het. 1, p. 34 (1884) (Heliura) (pr. p.).

scintillans Rothsch., Nov. Zool. 18, p. 44 (1911).

sixola Schaus, Ann. \& Mag. Nat. Hist. (8) 6, p. 190 (1910). sordida(-us) Rothsch., Nov. Zool. 18, p. 44 (1911).

thetis L., Mant. Plant. p. 539 (1771) (Sphinx). - Drury, Ill. Exot. Ent. 1, p. 57, t. 26, f. $4(1770)$. Hamps., Cat. Lep. Phal. 1, p. 386, f. 194 (1S98). thoos Fabr., Spec. Ins. 2, p. 166 (1781) (Zygaena). lenaeus Druce, Biol. Centr.-Am., Het. 1, p. 74 (1884) (Heliura) (pr. p.).

venata Butl., Illustr. Het. Br. Nius. 1, p. 49, t. 16, f. 7 (1877). - Schaus, Ain. Lep. p. 7, t. 1, f. 20 (1892). - Druce, Biol. Centr.-Am., Het. 2, p. 358 (1897). - Hamps., Cat. Lep. Phal. 1, p. 388 (1898).

a elia Schaus, Ent. Am. 5, p. 90 (1889) (Heliura). vinasia Schaus, Ann. \& Mag. Nat. Hist. (8) 6, p. 189 (1910). 29

\section{Androcharta Feld.}

Feld., Wien. Ent. Monatsschr. 6, p. 53 (1862). - Hamps., Cat. Cep. Phal. 1, p. 390 (1898).

eassotis Druce, Proc. Zool. Soc. Lond. p. 382 , t. 40 , f. 11 (1883). - Hamps., Cat. Lep. Phal. 1, p. 392 (1898).

diversipenn is Walk., List Lep. Ins. Br. Mus. 1, p. 225 (1854) (Euchromia). - Butl., Illustr. Het. Br. Mus. 1, p. 48 , t. 10, f. 9 (1877). - Hamps., Cat. Lep. Phal. 1, p. 392 (1898).

parvipennis Butl., Journ. Linn. Soc. Lond., Zool. 12, p. 427 (1876).

ab. brasiliensis Butl., Journ. Linn. Soc. Lond., Zool. 12, p. 427 (1876). - Hamps., Cat. Lep. Phal. 1, p. 393 (1898).

ab. clavipennis Butl., Trans. Ent. Soc. Lond. p. 46 (1878). - Hamps., Cat. Lep. Phal. I, p. 393 (1898).

gigantea(-um) Druce, Ann. \& Mag. Nat. Hist. (7) 3, p. 230 (1899).

meones Cram., Pap. Exot. 4, p. 73, t. 325, f. F. (1780) (Splinx). - Feld., Wien. Int. Monatsschr. 6, p. 53 (1862). - Hamps., Cat. Lep. Phal. 1, 1). 391 , f. 108 (1898).
Peru

Mexico,

Costarica,

Trinidad,

Venezucia,

Brit.- u.

Guayana, Peru

Brit.:

Honduras

Venezuela, Surinam, Amazonas

Costarica

Venezuela

Panama,

Venezuela,

Argentinien

Mcxico,

Amazonas

Costarica

Ecuador, Amazonas

Venezucla, Amazonas, Pará, São Paulo, Pcru

Fcuador

Colombia, Amazonas, Venczuela, Surinam
Tranz.- 
compta Sepp, Surin. Vlind. 1, p. 81, t. 37, (1848) (Glaucopis).

meonioides Herr.-Schäff., Aussereur. Schmett. 1, p. 74, f. 237 (1854) (Charidea).

Biologie: Scpp, Surin. Vlind. 1, p. 81, t. 37, (1848).

- Hahnel, Iris 3, p. 308 (1890).

rubricincta Burm., Descr. Phys. Rep. Argent. 5, p. 386 (1878) (Charidea). - Berg, An. Soc. Cient. Argent. 13, p. 178 (1882). - Hamps., Cat. Lep.

Amazonas, Phal. 1, p. 391 , t. 14 , f. 13 (1898).

Leechi Druco, Proc. Zool. Soc. Lond. p. 283 (1893). Stretehi(-ii) Butl., Journ. Linn. Soc. Lond., Zool. 12, p. 427 (1876); Illustr. Het. Br. Mus. 1, p. 48, t. 18, f. 7 (1877). - Hamps., Cat. Lep. Phal. 1, 6 p. 393 (1898).

Argentinien

Peru,

Rio Janciro,

(M.C.Vind.),

Uruguay

Eriphioides Kirby.

Kirby, Cat. Het. p. 159 (1892). - Hamps., Cat. Lep. Phal. 1, p. 393 (1898). Eriphia Butl., Journ. Linn. Soc. Lond., Zool. 12, p. 414, t. 29, f. 16 (1876) (nec IIerr.-Schäff.).

albipunctata Latliy, The Ent. 32, p. 119 (1899). surinamensis Möschl., Verl. zool.-bot. Ges. Wien 27, p. 640, t. 8 , f. 11, 11a (1877) (Eriphia). Hamps., Cat. Lep. Phal. 1, p. 394 (1898).

tractipennis Druce, Biol. Centr.-Am., Het. 1, p. 69 (1884) (Eriphia) (pr. p. context.).

tractipennis Butl., Journ. Linn. Soc. Lond. 12, p. 414 (1876) (Eriphia). - Druce, Biol. Centr.-Am., Het. 1, p. 69 (pr. p.), t. 7, f. 27 (1884). - Hamps., Cat. Lep. Phal. 1, p. 394 (1898).

ustulata Feld., Reise Nov., Lep. 2, p. 4, t. 102, f. 17 (1869) (Eriphia). - Hamps., Cat. Lep. Phal. 1, p. 395, f. 199 (1898).

v. columbina Dyar, Journ. New York Ent. Soc. 7, p. 175 (1899).

viridis Lathy, The Ent. 32, p. 118 (1899).

Ecuador

Panama,

Colombia,

Surinam

Honduras,

Guatemala,

Nicaragua,

Costarica,

Panama,

Rio Janeiro

(M.C.Vind.)

Colombia

Patria?

Ecuador 5

\section{Ceramidia Butl.}

But1., Journ. Linn. Soc. Lond., Zool. 12, p. 412, t. 29, f. 3 (1876). Hamps., Cat. Lep. Phal. 1, p. 395 (1898).

Passineura Butl., Journ. Linn. Soc. Lond., Zool. 12, p. 412, t. 29, f. 4 (1876).

Butleri Möschl., Verh. zool.-bot. Ges. Wien 27, p. 640, t. 8, f. 12, 12a (1877) (Eriphia). - Hamps., Cat. Lep. Phal. 1, p. 396, f. 200 (1898). ? Cockle, Can. Ent. 36, p. 204 (1904); 42, p. 95 (1910).

criphia Drucc, Biol. Centr.-Am., Het. 1, p. 68 (1884) (Antichloris) (nec Fabr.).

Guatemala, Venezucla, (II. C. Vind.), Surinam, Biologie: Cockle Can. Ent. 42, p. 95 (1910). 
caca Hübn., Zutr. Exot. Schmett. 1, p. 24, f. 133, 134 (1827) (Antichloris). - Hamps., Cat. Lep. Phal. 1, p. 396 (1898).

cntaleuca Butl., Journ. Linn. Soc. Lond., Zool. 12, p. 412 (1876). - Hamps., Cat. Lep. Phal. 1, p. 397 , t. 14, f. 5 (1898).

caurensis Klages, Proc. U. S. Nat. Mus. 29, p. 548 (1906). chalcoviridis Hamps., Ann. \& Mag. Nat. Hist. (7) 8, p. $172(1901)$.

chloroplegia Druce, Ann. \& Mag. Nat. Hist. (7) 15, p. 462 (1905).

cuprea Schaus., Journ. Ncw'York Ent. Soc. 9, p. 42 (1901).

eyanopasta Dogn., Hét. nouv. Am. Sud 2, p. 10 (1911).

flammea Dogn., Bull. Soc. Fint. France 60, p. CLV (1891) (Antichloris); Lép. Loja p. 82, t. 8, f. 2 (1894). - Hamps., Cat. Lep. Phal. 1, p. 398 (1898).

fumipenn is Walk., List Lep. Ins. Br. Mus. 1, p. 241 (1854) (Euchromia). - Hamps., Cat. Lep. Phal. 1, p. 398, t. 14, f. 26 (1898).

musicola Cockerell, Can. Ent. 12, p. 60 (1910) (Butleri var?).

obscura Butl., Illustr. Het. Br. Mus. 1, p. 40, t. 16, f. 5 (1877). - Hamps., Cat. Lep. Phal. 1, p. 396 (1898).

ornata Druce, Proc. Zool. Soc. Lond. p. 380 (1883) (Illipula). - Hamps., Cat. Lep. Phal. 1, p. 397 , t. 14 , f. 16 (189s).

phemonoides Möschl., Vorh. zool.-bot. Ges, Wion 27, p. 639 , t. 8, f. 10,10 a (1854) (Antichloris). Hamps., Cat. Lep. Phal. 1, p. 397 (1898).

fusiformis Butl., Journ. Linn. Soc. Lond., Zool. 12, p. 413 (1876) (Passineura) (nec Walk.).

viridis Druce, Biol. Centr.-Am., Het 1, p. 68, t. 7, f. 25 (1884) (Antichloris). - Dyar, Can. Ent. 42, 15
Rio Janeiro Argentinien

Ecuador, Ob.-

Amazonas, Surinam

Venezuela

Brasilien

Venezuela

Nexico

Venezuela

Colombia, Ecuador, Bolivia

Amazonas

Colorado (importata ex Honduras?

Amazonas

Colombia, Ecuador, Bolivia

Venczuela, Surinam, Pará

Panama

\section{Amyeles Herr.-Schäff.}

Herr.-Schäff., Aussereur. Schmett. p. 80 (1854). - Hamps., Cat. Lep. Phal. 1, p. 398 (1898).

Dipteromorpha Feld., Reise Nov., Lep. Atlas p. 4 (1869).

anthracina Walk., List Lep. Ins. Br. Mus. 1, p. 253 (1854) (Euchromia). - Herr.-Schäff., Aussereur. Schmett. p. 72, f. 259 (1854). — Butl., Illustr. Het. Br. Mus. 1, p. 40, t. 9, f. 7 (1877). - Druce, Biol. Centr.-Am., Het. 1, p. 68 (1884). - Hamps., Cat. Lep. Phal. 1, p. 398, f. 201 (1898). — Klages, Proc. U. S. Nat. Mus. 29, p. 548 (1906). adusta Feld., Reise Nov., Lep. 2, p. 4, t. 102 , f. 28 (1869) (Dipteromorpha). - Druce, Biol. Centr.-Am., Het. 1, p. 67 (1884).
Cubr (M. C.

Vind.),

Mexico,

Guatemala,

Panama,

Venezuela.

Brasilien

(ubi?) 
Biologie: Druce, Biol. Centr.-Am., Het. 1, p. 67 (1884). - Haase, Bibl. Zool. 8 (2), p. 76 (1893) (Mimicry).

dolosia Walk., List Lcp. Ins. Br. Mus. I, p. 238 (1854) Venezucla, (Euchomia). - Hmaps., Cat. Lep. Phal. 1, Pernam2 p. 399 , f. 14, f. 6 (1898).

buco

\section{Antichloris Hiubn.}

Hübn., Ver\%. bek. Schmctt. p. 124 (1826). - Butl., Journ. Linn. Soc. Lond., Zool. 12, p. 413, t. 29, f. 5 (1876). - Hamps., Cat. Lep. Phal. 1, p. 399 (1898).

Illipula Walk., List Lcp. Ins. Br. Mus. 1, p. 237 (IS54). - But]., Journ. Linn. Soc. Lond. Zool. 12, p. 410, t. 29, f. 2 (1876).

Eriphia Herr.-Schäff., Aussereur. Schmctt. p. 16 (1854) (nec Mcig. 1838).

eriphin Fabr., Gen. Ins. p. 276 (1776) (Zygaena). Burm., Deser. Phys. Rep. Argent. 5, p. 388 (1878). - Hamps., Cat. Lep. Phal. I, p. 400, f. 203 (1898). - Schrottky Iris 24, p. 152 (1910). alecton Cram., Pap. Exot. 4, p. 181, t. 382, f. D (1782) (Sphinz).

phemonoe Hübn., Zutr. Exot. Schmett. 1, p. 9, f. $15,16(1827)$.

melanochloros Sepp, Surin. Vlind. 2, p. 145, t. 69 (1848) (Sesia).

scapularis Herr.-Schäff., Aussereur. Schmett. f. 260 (1856) (Amycles).

helus Herr.-Schäff., Aussereur. Schmett. p. 74, f. 263 (1856) (Charidea).

Biologie: Sepp, Surin. Vlind. 2, p. 145, t. 69 (1848). - Schrottky, Iris 24, p. 250 (1910).

panace a Druce, Biol. Centr.-Am., Het. 1, p. 68, t. 8, f. 17 (1884). - Hamps., Cat. Lep. Phal. 1, p. 401, f. 204 (1898).

puriseal Schaus, Ann. \& Mag. Nat. Hist. (8) 7, p. 181 (1911).

purpurea Lathy, The Ent. 32, p. 119 (1899).

quadricolor Walk., List Lep. Ins. Br. Mus. 35, p. 1867 (1866) (Charidea). - Hamps., Cat. Lep. Phal. I, p. 399 , f. 202 (1898).

Quartzi Klages, Proc. U. S. Nat. Mus. 29, p. 548 (1906).

Seudueri(-ii) Butl., Journ. Linn. Soc. Lond., Zool. 12, p. 413 (1876). - Hamps., Cat. Lep. Phal. 1, 7 p. 400 , t. 14 , f. 25 (1898).

Trinidad,

Venezueja,

Brit.-

Guayari,

Surinam,

Amazonas

(M.C.Vind.),

Pará

Pernam-

buco, Rio

Janeiro,

Santa

Catharina

(M.C.Vind.), Paraguay,

Bolivia, (M.C.Vind.)

Costarica

Ecuador

Jamaica

Venezuela

Pará

\section{Methysia Butl.}

Butr., Journ. Linn. Soc. Lond., Zool. 12, p. 397 (1876). — Hamps., Cat. Lep. Phal. 1, p. 401 (1876).

hilda Druce, Ann. \& Mag. Nat. Hist. (7) 18, p. 79 (1906). Peru melanota Hamps., Ann. \& Mag. Nat. Hist. (8) 4, p. 347 Brit.(1909). 
notabilis Walk., List Lep. Ins. Br. Mus. 1, p. 144 (1854)

(Glaucopis). - But1., Illustr. Het. Br. Mus. 1,

Pará

p. 36, t. 7, f. 11 (1877). - Hamps., Cat. Lep. 3

Phal. 1, p. 401, f. 205 (1898).

\section{Pseudaclytia Butl.}

Butl., Journ. Linn. Soc. Lond., Zool. 12, p. 409 (1876). - Hamps., Cat. Lep. Phal. 1, p. 402 (1898).

flavidorsia Hamps., Ann. \& Mag. Nat. Hist. (7) 19, p. 226 Venezuela (1907).

major Druce, Ann. \& Mag. Nat. Hist. (7) 18, p. 79 (1906). minor Schaus, Proc. U. S. Nat. Mlus. 29, p. 189 (1905).

opponens Walk., List Lep. Ins. Br. Mus. 31, p. 100 (1864) (Pampa). - Hamps., Cat. Jep. Phal. 1, p. 402, f. 207 (1898).

rufogularis Möschl., Stett. Ent. Zeitg. 33, p. 349 (1872) (Charidea).

umbrica Druce, Ann. \& Mag. Nat. Hist. (7) 1, p. 208 (1898). - Hamps., Cat. Lop. Phal. 1, p. 402, f. 206 (1898).

unimacula Schaus, Proc. U. S. Nat. Mus. 29, p. 189 (1905).

6

Peru

Franz:-

Guayana

Amazonas

\section{Brasilien}

(Cabo)

Franz.-

Guayana

\section{Atyphopsis Butl.}

Butl., Trans. Ent. Soc. Lond. p. 49 (1887). - Hamps., Cat. Lep. Phal. 1, p. $403(1898)$.

modesta Butl., Trans. Ent. Soc. Lond. p. 49 (1878).
Hamps., Cat. Lep. Plial. 1, p. 403, f. 208 (1898).

obscura Hamps., Cat. Lep. Plaal. 1, p. 404, t. 14, f. 7 (1898).

roseiceps Druce, Ann. \& Mag. Nat. Hist. (7) 1, p. 408 (1898). - Hamps., Cat. Lep. Phal. 1, p. 403, 3 t. 14, f. 27 (1898).

Amazonas

Espiritu Santo

Rio Janeiro

\section{Amaphlebia Feld.}

Fold., Roisc Nov., Lep. Atlas p. 1 (1869). - Hamps., Cat. Lep. Phal. 1, p. 404 (1898).

caudatula Feld., Reise Nov., Tep. 2, p. 1, t. 102, f. 36 (1869). - Hamps., Cat. Lep. Phal. 1, p. 404, 1 f. 209 (1898).

Amazonas

\section{Sciopsyche Butl.}

Butl., Journ. Linn. Soe. Lond., Zool. 12, p. 426, t. 29, f. 20 (1876). - Hamps.

Cat. Lep. Pinal. 1, p. 404 (1898).

aurantiocauda (auranticanda) Klages, Proc. U. S. Nat. Venczucla Mus. 29, p. 519 (1906).

remissa Dogn., Ann. Soc. Ent. Belg. 46, p. 229 (1902). " 
tropiea Walk., List Lep. Ins. Br. Mus. 1, p. 236 (1854) (Euchromia). - Hamps., Cat. Lep. Phal. 1, p. 405. f. 210 (1898).

cinerea Butl., Journ. Linn. Soc. Lond., Zool. 12, p. 426 (1876).

? Honduras, Colombia, Amazonas, Pará, Espiritu Santo 3

\section{Napata Walk.}

Walk., List Lep. Ins. Br. Mus. 1, p. 231 (1854). - Hamps., Cat. Lep. Phal. 1, p. 405 (1898).

Diabaena Feld., Reise Nov., Lep. Atlas p. 4 (1869).

Metriophyla Butl., Illustr. Het. Br. Mus. 1, p. 43 (1877).

albimneulata Hamps., Ann. \& Mag. Nat. Hist. (7) 8, Panama p. $172(1901)$.

albiplaga Walk., List Lep. Ins. Br. Mus. I, p. 218 (1854) (Euchromia). - Hamps., Cat. Lep. Phal. 1, p. 409, f. 212 (1898).

a picalis Herr.-Schäff.: Aussereur. Schmett. p. 74, f. 236 (1854) (Charidea). - Butl., Journ. Linn. Soc. Iond., Zool. 12, p. 417 , t. 29 , f. 9 (1876); Illustr. Hot. Br. Mus. 1, p. 43, t. 8, f. 7 (1877). - Druec, Biol. Centr.-Am., Het. 1, p. 73 (1884); 2, p. $353(1897)$.

lelex Druce, Proc. Zool. Soc. Lond. p. 495 (1890) (Heliuira) (ठ̃).

atalanta Druce, Ann. \& Mag. Nat. Hist. (7) 3, p. 230 (1899).

atricincta Hamps., Ann. \& Mag. Nat. Hist. (7) 8, p. 172 (1901).

Bocttgeri Druce, Ann. \& Mag. Nat. Hist. (8) 3, p. 457 (1909).

Broadwayi Schaus, Journ. New York Ent. Soc. 4, p. 130 (1896) (Syntomeida). - Hamps., Cat. Lep. Phal. 1, p. 413 , f. 213 (1898).

castra Hamps., Cat. Lep. Plal. 1, p. 409, t. 14, f. 14 (1898).

chalybea Hübn., Zutr. Exot. Schmett. 3, p. 14, f. 439, 440 (1827) (Uranophora).

eincticollis Feld., Reise Nov., Lep. 2, p. 4, t. 103, f. I (1869) (Diabaena ). - Hamps., Cat. Lep. Phal. I, p. 412 (1898).

cuchloa Hamps., Ann. \& Mag. Nat. Hist. (7) 15, p. 429 (1905).

chalybea Hamps., Cat. Lep. Phal. 1, p. 409 (1898) (nec Hübn.).

Felderi Zerny nom. nor.

eucyane Fold., Reiso Nov., Lep. 2, p. 3, t. 102, f. 16 (1869) (Cyanopepla) (nec f. 14). - Hamps., Cat. Lep. Phal. 1, p. 407 (1898).

fenestrata Druce, Ann. \& Mlag. Nat. Hist. (6) 18, p. 30 (1896) (Laemocharis). Hamps., Cat. Lep. Phal. 1, p. 405, f. 211 (1898).

Ilaviceps Hamps., Ann. \& Mag. Nat. Hist. (7) 8, p. 173 (1901).

guatemalena Druce, Biol. Centr.-Am., Het. 1, p. 66 (1884); 2, p. 351, t. 72, f. 6 (1897). - Hamps., Cat. Lep. Phal, 1, p. 411 (I898).

Inexieo,

Nicaragua,

Costarica,

Panama,

Venczuela,

Ecuador,

Rio Janeiro

(M.C.Vind.)

Amazonas

Bahia

Peru

Trinidad

Paraná

Cuba

Colombia

Jamaica

Brasilien

(ubi?)

São Paulo

Panama

Mexico, Guatemala 
iridis Hamps., Cat. Lep. Phal. 1, p. 408, t. 14, f. 10 (1898). jynx Hübn.-Gey., Zutr. Exot. Schmett. 4, p. 39, f. 781, 782 (1832) (Calosoma). - Hamps., Cat. Lep. Phal. 1, p. 408 (1898).

lelex Druce, Proc. Zool. Soc. Lond. p. 495 (1890) (Heliura) (9); Biol. Centr.-Am., Hct. 2, p. 353 (1897). - Hamps., Cat. Lep. Phal. 1, p. 410, t. 14, f. 28 (1898).

lena Schaus, Proc. Zool. Soc. Lond. p. 277 (1892) (Mctriophyla); Am. Lep. p. 7, t. 1, f. 24 (1892). Hamps., Cat. Lep. Phal. 1, p. 412 (1898).

leueotela(-us) Butl., Journ. Linn. Soe. Lond., Zool. 12, p. 409 (1876). - Druce, Biol. Centr. -Am., Het. 1, p. 66, t. 7 , f. 24 (1884); 2, p. 350 (1897). Hamps., Cat. Lep. Phal. 1, p. 411 (1898).

metamelaena (metamcla) Dogn., Hét. nouv. Am. Sud 3. p. 4 (19I1).

uunda Walk., List Lep. Ins. Br. Mus. 7, p. 1680 (1856) (Ardonea). - Hamps., Cat. Lep. Phal. 1, p. 412, t. 14 , f. 9 (1898).

quadrimacnIata Möschl., Stett. Ent. Zeitg. 33, p. 349 (1872) (Euagra); Verh. zool.-bot. Ges. Wien 27, t. 9, f. 24 (1877). - Hamps., Cat. Lep. Phal. 1, p. 412 (1898).

qual ristrigata Hamps., Cat. Lep. Phal. 1, p. 409, t. 14, f. 8 (1898).

rhabdonotu (rabdonota) Hamps., Cat. Lep. Phal. 1, p. 410, t. 14, f. 11 (1818).

sanguieineta Hamps., Ann. \& Mag. Nat. Hist. (7) 8, p. $173(1901)$

splendida Herr.-Schäff., Ausscreur. Schmett. p. 74, f. 232 (1854) (Charidea). - Hamps., Cat. Lcp. Phal. 1, p. 407 (1898).

eucyane Fcld., Reisc Nor. Lep. 2, p. 4, t. 102, f. 14 (1869) (Entomis).

argentiflua Martyn, Psyche t. 29, f. 82, 83 (1797) (non ed.); Exp. Ausl. Schmett. Sphing. exot. t. 2, adscit. t. 1, f. 1 (1830) (non ed.). - Möschl., Verh. zool.-bot. Ges. Wien 27, p. 641 (1877).

subflareseens Kaye, The Ent. 44, p. 143 (1911).

superba Druce, Ann. \& MIag. Nat. Hist. (7) 18, p. 79 (1906)

terminalis Walk., List Lep. Ins. Br. Mus. 1, p. 231 (1854) (Euchromia). - Hainps., Cat. Lep. Phal. 1, p. 411 , t. 14 , f. 12 (1898).

unifaseia Schaus, Journ. New York Ent. Soc. 6, p. 140 (1898). - Hamps., Cat. Lep. Phal. 1, p. 408, t. 14 , f. 15 (1898).

venezuelensis Kllages, Proc. U. S. Nat. Mus. 29, p. 549 (1906).
Bolivia

Amazonas (M.

C. Vind.),

Paraná,

Santa

Catharina

(M.C.Vind.)

Mexico,

Guatemala,

Panama,

Colombia,

Venezucla,

Ecuador

Peru

Mexico,

Guatemala,

Brit. -

Honduras,

Honduras,

Costarica,

Venezuela,

Surinam

Franz.-

Guayana

Haiti

Venezuela,

Surinam,

Franz.-

Guayana

Santa Lucia, Dominica

Dominica

Peru

Colombia, Venezucla, Surinam,

Franz. -

Guayana,

Bolivia,

Santa

Catharina

Brit. -

Guayana

Peru

Pcrnambuco

Ecuador

Venezucla 
Walkeri Druce, Ann. \& Mag. Nat. Hist. (6) 4, p. 86

(1889) (Evius); Biol. Centr.-Am., Het. 2, p. 365,

t. 73, f. 21 (1897). - Hamps., Cat. Lep. Phal. 1, 32 p. 407 (1898).

Costarica, Panama (I. Taboga), Trinidad

\section{Trichromia Hübn.}

Hübn., Verz. bek. Schmett. p. 164 (1826). - Hamps., Ann. \& Mag. Nat. Hist. (7) 15, p. 429 (1905).

Pa.treliura Hamps., Cat. Lep. Phal. 1, p. 413 (1898).

capys Cram., Pap. Exot. 1, p. 88, t. 56, f. E (1775) Brit.(Sphinx). - Hamps., Cat. Lep. Phal. 1, p. 413, Guayana, f. 214 (1898).

lacteinota Butl., Journ. Linn. Soc. Lond., Zool. 12, p. 417 (1876) (Heliura).

1

Surinam,

Amazonas, Pará

\section{Lymixe Walk.}

Walk., List Lep. Ins. Br. Mus. 2, p. 489 (1854). - Hamps., Cat. Lep. Phal. 1, p. 414 (1898).

Mallostethus Butl., Journ. Linn. Soc. Lond., Zool. 12, p. 408 (1876).

albipennis Herr.-Scliäff., Corresp.-Bl. zool.-min. Ver. Regensb. 20, p. 117 (1866) (Echeta). - Hamps., Cat. Lep. Phal. 1, p. 416, t. 14, f. 20 (1898).

Edwardsi Grote, Papilio 1, p. 4 (1881) (Scepsis). Neum. \& Dyar, Joum. Now York Ent. Soc. 1, p, 104 (1893). - Hamps., Cat. Lep. Phal. 1, p. 415, t. 14, f. 18 (1898). - Holl., Moth. Book p. 100 , t. 13 , f. 11 (1903). - Hanps., Ann. \& Mag. Nat. Hist. (7) 14 , p. 165 (1904).

Biologie: Hy. Edw., Lnt. Am. 3, p. 167 (1887). - Dyar, Ins. Life 2, p. 361 (1880); Proc. Boston Soc. Nat. Hist. 27, p. 138 (1896). - Hamps., Cat. Lep. Phal. 1, p. 415 (1898).

melanoeephala Walk., List Lep. Ins. Br. Mus. 2, p. 490 (1854). - Hamps., Cat. Lep. Phal. 1, p. 415, t. 14 , f. 19 (1898).

metamelaena(-mclas) Walk., List Lep. Ins. Br. Mus. 1, p. 145 (1854) (Glaucopis). - But1., Illustr. Het. Br. Mus. 1, p. 39, t. 7, f. 10 (1877). - Hamps., Cat. Lep. Phal. 1, p. 414, f. 215 (1898).

strigivenia Druce, Ann. \& Mag. Nat. Hist. (7) 1, p. 407 (1898). - Hamps., Cat. Lep. Phal. 1, p. 415, 5 t. 14 , f. 17 (1898).

Cuba

Florida,

Bahama-I.

Jamaica

Pará

Franz.

Guayana

Loxozona Hamps.

Hamps., Cat. Lep. Phal. 1, p. 416 (1898).

aeuminata Walk., List Lep. Ins. Br. Nus. 7, p. 1629 (1856) (Pampa). - Hamps., Cat. Lep. Phal., 1, p. 417 (1898).

Brasilien

(ubi?) 
Hartmanni Schaus, Proc. Zool. Soc. Lond. p. 280

(1892) (Sycheria); Am. Lcp. p. 11, t. 3, f. 10 (1892).

lanceolata Walk., I.ist Lep. Ins. Br. Mus. 2, p. 307 (1854) (Josia). - Hamps., Cat. Lep. Phal. 1, 2 p. 416 , f. 216 (1898).

Pará

\section{Horama Hübn.}

Hübn., Verz. bek. Schmett. p. 124 (1826). - Hamps., Cat. Lep. Phal. 1, p. 417 (1898).

Mastigocera Harr., Am. Journ. Sc. 36, p. 289, 315 (1839).

Phylloecia Guér., Icon. Régne Animal Ins. p. 504 (1843).

Callicarus Grote, Proc. Ent. Soc. Philad.6, p. 182 (1867).

Drucea Kirby, Cat. Het. p. 130 (1892).

Biologie: Haase, Bibl. Zool. 8 (2), p. 77 (1893) (Mimicry).

castrensis Jones, Trans. Ent. Soc. Lond. p. 144 (1908). clavipes Boisd., Iép. Guatem. p. 81 (1870) (Mastigocera).

- Druce, Biol. Centr.-Am., Het. 1, p. 49, t. 6, f. 20 (1884); 2, p. 339 (1897). - Hamps., Cat. Lop. Phal. 1, p. 420, f. 219 (1898).

diffissa Grote, Proc. Ent. Soc. Philad. 6, p. 181, 310 t. 5, f. 2 (1886). - Hamps., Cat. Lep. Phal. 1, p. 421 (1898).

pretellus Herr.-Schäff., Corresp.-Bl. zool.-min. Ver. Regensb. 20, p. 113 (1866).

flarata Jones, Trans. Ent. Soc. Lond. p. 144 (1908).

flavicans Dogn., Hét. nouv. Am. Sud 2, p. Il (1911).

Grotei Butl., Journ. Linn. Soc. Lond. 12, p. 374 (1876). - Hamps., Cat. Lep. Phal. 1, p. 421, f. 221 (1898).

oedipus (oedippus) Boisd., Lép. Guatem. p. 81 (1870) (Mostigocera). - Druce, Biol. Centr.-Am., Het. 1, p. 49, t. 6, f. 19 (1884); 2, p. 339 (1897). - Hamps., Cat. Lcp. Phal. 1, p. 418 (1898).

Biologie: Haase, Bibl. Zool. (8) 2, p. 77 (1893) (Mimicry). - Druce, Biol. Centr.-Am., Het. 2, p. 339 (1897) (Mimicry).

pantalon (panthalon) Fabr., Syst. Ent. p. 405 (1793) (Zygaena). - Hamps., Cat. Lep. Phal. 1, p. 417, t. 15 , f. 9 (1898).

tibialis Butl., Journ. Linn. Soc. Lond., Zool. 12, p. 373 (1876) (Mastigocera).

pennipes Grote, Proc. Ent. Soc. Philad. 6, p. 182 (1867) (Callicarus). - Hamps., Cat. Lep. Phal. 1, p. 419 (1898).

plumipes Drury, Ill. Exot. Ent. 2, p. 47, t. 27, f. 3 (1773) (Sphinx). - Butl., Illustr. Hot. Br. Mus. 1, p. 25, t. 8, f. 2 (1877). - Druce, Biol. Centr.-Am., Het. 1, p. 49 (1884); 2, p. 338 (1897). - Hamps., Cat. Lep. Phal. 1, p. 419, f. 218 (1898).

punctata Gnér., Icon. Régne Animal, Ins. p. 504 (1843) (Phylloecia). — Druce, Biol. Centr.-Am., Het. 2, p. 338 (1897).

jalapensis Neum., Ent. Am. 6, p. 63 (1890). Druce, Biol. Centr.-Am., Het. 2, p. 339 (1897).

Paraná

Mexico, Guatemala, Costarica, Venezucla Cuba

Paraná

Colombia

Jamaica

Mexico, Guatemala

Haiti, Venczuela

Cuba

Mexico, Honduras

Guatemala, Nicaragua 
pretus Cram., Pap. Exot. 2, p. 121, t. 175, f. E, F (1777) (Sphinx). - Hübn., Samml. Exot. Schmett. 2 (1827). - Hamps., Cat. Lep. Phal. 1, p. 422, f. 222 (1898).

eumolphus Fabr., Spec. Ins. 2, Append. p. 505 (1787) (Zygaena).

vespina Harr., Am. Journ. Sc. 36, p. 315 (1839) (Mastigocera).

Biologic: Haasc, Bibl. Zool. 8 (2), p. 77 (1893) (Mimicry).

tarsalis Walk., List Lep. Ins. Br. Mus. 7, p. 1633 (1856). - Hamps., Cat. Lop. Phal. 1, p. 420, f. 220 (1898).

texana(-us) Grote, Proc. Ent. Soc. Philad. 6, p. 184 (1867) (Callicarus). - Druec, Biol. Centr.-Am., Het. 1, p. 49 (1884); 2, p. 338 (1897). - Ncum. \& Dyar, Journ. Netw York Ent. Soc. 1, p. 100

St. 'Thomas, Venczuela (1893). - Hamps., Cat. Lep. Phal. 1, p. 418, f. 217 (1898). - Holl., Moth Book p. 100, f. 13, 13 f. $9(1903)$

Patria?

Texas, Mexico, Honduras

Empyreuma Hübn.

Hübn., Verz. bck. Schmett. p. 120 (1826). - Butl., Journ. Linn. Soc. Lond. 12, p. 361, t. 28, f. 19 (1877). - Hamps., Cat. Lep. Phal. 1, p. 422 (1893).

muero Zerny nom. nov.

lichas Fabr., Spec. Ins. 2, Appond. p. 505 (1781) (Zygaena) (nce Cram. 1775). - Hamps., Cat. Lep. Phal. 1, p. 423, f. 223 (1898). pugione Ilübn., Zutr. Exot. Schmett. 1, p. 12, f. 41,42 (1827) (neo L.).

sanguinosa Martyn, Psyche t. 26, f. 18, 19 (1797)

(Sphinx) (non ed.). - Druce, Biol. Centr.-Am., Het. 2, p. 334 (1896).

pugione L., Syst. Nat. ed. 12, p. 807 (1767) (Sphinx). - Hamps., Cat. Lep. Phal. 1, p. 423 (1898).

lichas Cram., Pap. Exot. 1, p. 70 , t. 45, f. B

Bahama-I., Haiti, Cuba, Honduras

Jamaica, St. Thomas (1775) (Sphinx).

Biologie: Hamps., Cat. Lep. Phal. 1, p. 22, f. 8, 2 p. 423 (1898).

Harecidia Schaus.

Schaus, Journ. Now York Ent. Soc. 9, p. 43 (1901). sanguipuncta Sohaus, Journ. Netw York Ent. Soc. 9, Santa 1 p. 43 (1901).

Catharina

\section{Teucopleura Hamps.}

Hamps., Cat. Lep. Phal. 1, p. 423 (1898).

cucadma Drucc, Ann. \& Mag., Nat. Hist. (6) 13, p. 171, Panama (1894) (Cosmosoma); Biol. Centr.-Am., Het. 2, p. 335 , t. 70, f. 22 (1896). - Hamps., Cat. Lep. Phal. I, p. 424, f. 224 (1898). 
Psendophenoptera Butl.

Butl., Journ. Linn. Soc. Lond., Zool. 12, p. 370, t. 28, f. 4 (1876). Hamps., Cat. Lep. Phal. 1, p. 424 (1808).

Chloropsinus Butl., Journ. Linn. Soc. Lond., Zool. 12, p. 409, t. 29 , f. 1 (1876).

basalis Walk., List Lep. Ins. Br. Mus. 1, p. 251 (1854) (Euchromia). - Hamps., Cat. Lep. Phal. 1, p. 424, f. 225 (1898); Ann. \& Mag. Nat. Hist.

Amazonas, (7) 15, p. 427 (1005).

lanceolatus Butl., Journ. Linn. Soc. Lond., Zool. 12, 1

p. 409 (1876) (Chloropsinus).

Pseudopompilia Hamps.

Hamps., Cat. Lep. Phal. 1, p. 425 (1898).

mimica Druce, Ann. \& Mag. Nat. Hist. (7) 1, p. 401 (1898). - Hamps., Cat. Lep. Phal. 1, p. 425, f. 226 1 (1808).

Xanthoplen'a Hamps.

Hamps., Cat. Lop. Phal. 1, p. 426 (1898).

? flavocineta Guér., Icon. Régne Animal, Ins. p. 503 (1813) (Glaucopis). - Hamps., Cat. Lep. Phal. 1, p. 427 (1898) (huius generis?).

perspicua Walk., List Lep. Ins. Br. Mus. 7, p. 1627 (1856) (Calonotos). - Hamps., Cat. Lep. Phal. 1, p. 426 (1898).

sandion Druce, Proc. Zool. Soc. Lond. p. 373 (1883) (Calonotos).

Troctschi (Trotschi) Druce, Biol. Centr.-Am., Het. 1, p. 44, t. 8, f. 8 (1884) (Calonotos). - Hamps., 3 Cat. Lep. Phal. 1, p. 426, f. 227 (1898).

Amazonas

Franz.-

Guayana

Colombia,

Ecuador,

Amazonas,

Pará

Panama

Chlorostola Hamps.

Hamps., Cat. Lcp. Phal. 1, p. 427 (1898).

corydon Druce, Biol. Centr.-Am., Het. 1, p. 44, t. 6, f. 13 (1884); 2, p. 333 (1896) (Eupyra). - Hamps., Cat. Lep. Phal. 1, p. 428, f. 228 (1898).

interrupta Walk., List Lep. Ins. Br. MIus. 7, p. 1628 (1856) (Calonotos). - Druce, Biol. Ccntr.-Am., Het. 1, p. 44 (1884). - Hamps., Cat. Lep. 2 Phal. 1, p. 427 , t. 15, f. 13 (1898).

Mexico,

Guatemala

Mexico

\section{Ixylasia Butl.}

Butl., Journ. Linn. Soc. Lond., Zool. 12, p. 410, t. 29, f. 8 (1876). Hamps., Cat. Lep. Phal. 1, p. 428 (1898).

Kelleri Klagcs, Poc. U. S. Nat. Mus. 29, p. 550 (1906). Venezuela pyroproct is Druce, Ann. \& Mag. Nat. Hist. (7) 15, p. 462 (1905). 
Schausi Druce, Biol. Centr.-Am., Het. 2, p. 334, t. 70, f. 21 (1896) (Eupyra). - Hamps., Cat. Lep. Phal. 1, p. 429, f. 230 (1898).

semivitreata Hamps., Ann. \& Mag. Nat. Hist. (7) 15, p. 429 (1905).

almon Hamps., Cat. Lep. Phal. 1, p. 432, f. 233 (1898) (Autochloris) (nec Cr.).

trogonoides Walk., List Lep. Ins. Br. Mus. 31, p. 101 (1864) (Aclytia). - Hamps., Cat. Lep. Phal. 1, 5 p. 429 , f. 229 (1898).

Mexico

Rio Janeiro, Santa Catharina

Brasilien (ubi?)

\section{Phaenarete Druce.}

Druce, Ann. \& Mag. Nat. Hist. (6) 18, p. 31 (1896); Biol. Centr.-Am., Het. 2, p. 348 (1897). - Hamps., Cat. Lep. Phal. 1, p. 430 (1898).

diana Druce, Ann. \& Mag. Nat. Hist. (6) 18, p. 31 (1896); Panama Biol. Centr.-Am., Het. 2, p. 349, t. 72, f. 1 (1897). - Hamps., Cat. Lep. Phal. 1, p. 430, f. 231 1 (1898).

\section{Cacostatia Hamps.}

Hamps., Cat. Lep. Phal. 1, p. 430 (1898).

discalis Walk., List Lep. Ins. Br. Mus. 7, p. 1651 (1856) (Euagra). - Hamps., Cat. Lep. Phal. 1, p. 432, t. 15, f. $2(189 \mathrm{~s})$.

flavirentralis Dogn., Ann. Soc. Ent. Belg. 53, p. 215 (1909).

ossa Druce, Proc. Zool. Soc. Lond. p. 285 (1893) (Metastatia); Biol. Centr.-Am., Het. 2, p. 359, t. 73 , f. 1 (1897). - Hamps., Cat. Lep. Phal. 1, p. 431 (1898).

sapphira (saphira) Stdgr., Verh. zool.-bot. Ges. Wien 25, p. 122 (1875) (Agyrta). - Druce, Biol. Centr.Am., Het. 1, p. 81 (1884); 2, p. 359 (1897). Hamps., Cat. Lep. Phal. 1, p. 431, f. 232 (1898). umbraticola Klages, Proc. U. S. Nat. Mus. 29, p. 551 (1906).

Amazonas

Colombia

Panama, Brit.- u.

Franz.Guayana, Colombia

Mexico, Guatemala, Panama

Verezuela 5

\section{Schasiura Butl.}

Butl., Illustr. Het. Br. Mus. 1, p. 37 (1877). - Hamps., Ann. \& Mag. Nat. Hist. (7) 15 , p. 430 (1905).

A utochloris Hamps., Cat. Lep. Phal. 1, p. 432 (1898) (pr. p.) (nec Hübn.).

gymnelioides Zerny, Tris 26 (1912) (ined.).

Südamerika (ubi?)

nimica Bntl., lllustr. Het. Br. Mus. 1, p. 37, t. 16, f. 6 Amazonas (1877). - Hamps., Cat. Lep. Phal. 1, p. 433, f. 234 (1898). 
Gupyra Herr.-Schäff.

Herr.-Schäff., Aussereur. Schmett. p. 78 (1853). - Hamps., Cat. Lep. Phal. 1, p. 433 (1898).

consors Schaus, Proc. Zool. Soc. Lond. p. 273 (1892).; Peru, Arn. Lep. p. 2, t. 1, f. 7 (1892). - Hamps., Cat. Bolivia Lep. Phal. 1, p. 435 , f. 236 (189S).

disticta Hamps., Cat. Lep. Phal. 1, p. 433, t. 15, f. 15 Bolivia (1898).

dorata Dogn., Ann. Soc. Ent. Belg. 41, p. 23 (1897) (huins generis?).

imperialis Herr.-Schäff., Ausscreur. Schmett. p. 73, f. 54 (1858) (Ginucopis). - Hamps., Cat. Lcp. Phal. 1, p. 434, f. 235 (1898).

florella Butl., Ann. \& Hag. Nat. Hist. (4) 12, p. 227 (1873) (Chrysocale).

v. ducalis Maasson in Stübel, Reiso Südamcrika p. 43 (1890). - Hamps., Cat. Lep. Phal. 1, p. $434(1898)$.

psittacus Schaus, Proc. Zool. Soc. Lond. p. 273 (1892); Am. Lep. p. 1, t. 1, f. 6 (1892). - Hamps., Cat. Lep. Phal. I, p. 436 (1898).

sages Druce, Am. \& Mag. Nat. Hist. (6) 15, p. 43 (1895). - Hamps., Cat. Lep. Phal. 1, p. 435 , t. 15, f. 3 (1898).

sarama Dogn., Lc Nat. 13, p. 109 (1891). - Hamps., Venezuela 7 Cat. Lep. Plial. 1, p. 435, t. 15, f. 22 (1898).

Ecuador

Venezuela, Peru

Colombia

Peru,

Bolivia

Bolivia

Diaxanthia Hamps.

Hamps., Cat. Lep. Phal. 1, p. 436 (1898).

Iucinia Druce, Biol. Centr.-Am., Het. 1, p. 75, t. 8, f. 26 Costarica (1884) (Automolis). - Hamps., Cat. Lep. Plial. 1, 1 p. 436 , f. 237 (1898).

Tipulodes Boisd.

Boisd., Voy. Astrolabe, Lép. p. 195 (1832). - Hamps., Cat. Lep. Phal. 1, p. 437 (1898).

- ima Boisd., Voy. Astrolabe, Lép. p. 196, t. 3, f. 7 (1832). São Paulo - Hamps., Cat. Lep. Phal. 1, p. 437, f. 238 (M.C.Vind.), (1898).

1

Rio Janciro, Espiritu

Santo,

Rio Grande do Sul,

Paraguay

\title{
Callopepla Hamps.
}

Hamps., Cat. Lep. Phal. 1, p. 437 (1898).

emarginata Walk., List Lep. Ins. Br. Mus. 2, p. 295 (1854) (Josia). - Hamps., Cat. Tep. Phal. 1, p. 438 , t. 15 , f. $10(1898)$.

\author{
Amazonas \\ (M.C.Vind.), \\ Matto \\ Groeso (M. \\ C. Vind.), \\ S̃̃o Paulo
}


Ilammula Hübn.-Gey., Zutr. Exot. Schmett. 4, p. 14, Espiritu f. 637, 638 (1832) (Calosoma). - Hamps., Cat. Santo Lep. Phal. 1, p. 438 (1898).

inachia Schaus, Proc. Zool. Soc. Lond. p. 277 (1892) São Paulo (Charidea); Am. Lep. p. 6, t. 1, f. 19 (1892). (M. G. - Hamps., Cat. Lep. Phal. 1, p. 438, f. 239 Vind.), (1898).

katima Schaus, Journ. New York Ent. Soc. 4, p. 133 (1896) (Charidea).

? similis Heyl., C. R. Soc. Ent. Belg. 34, p. XV (1890) Rio Janeiro, Santa Catharina, Paraguay (Charidea). - Hamps., Cat. Lep. Phal. 1, 4 p. 438 (1898) (huius generis?).

São Paulo

\section{Cyanopepla Clem.}

Clem., Proc. Acad. Philad. p. 545 (1860). - Hamps., Cat. Lep. Phal. 1, p. 439 (1898).

Charidea Butl., Journ. Linn. Soc. Lond., Zool. 12, p. 415, t. 29, f. 11 (1876) (nec Dalm.). - Burm., Descr. Phys. Rep. Argent. 5, p. $382(1878)$.

agyrtidia Hamps., Cat. Lep. Phal. 1, p. 450, t. 15, f. 21 (1898).

alonzo Butl., Journ. Linn. Soc. Lond., Zool. 12, p. 415 (1876) (Charidea); Illustr. Het. Br. Mus. 1, p. 42 , t. 10 , f. 11 (1877). - Hamps., Cat. Lep. Phal. 1, p. 443 (1898).

imogena Butl., Journ. Linn. Soc. Lond., Zool. 12, p. 415 (1876) (Charidea).

amata Druce, Proc. Zool. Soc. Lond. p. 494 (1890) (Charidea). - Hamps., Cat. Lep. Phal. 1, p. 448, t. 15, f. 27 (1898).

arrogans Walk., List Lep. Ins. Br. Mus. 1, p. 214 (1854) (Euchromia). - Butl., Illustr. Hot. Br. Mus. I, p. 42 , t. 13, f. 12 (1877). - Druce, Biol. Centr.Am., Het. 1, p. 71 (1884). - Hamps., Cat. Lep. Phal. 1, p. 444 (1898).

ab. costaricensis Druce, Biol. Centr.-Am., Het. 1, p. 71 , t. 8, f. 20 (1898) (Charidea). - Hamps., Cat. Lep. Plial. 1, p. 444 (1898).

basimacula Hamps., Cat. Lep. Phal. 1, p. 449, t. 15, f. 24 (1898).

bella Guér., Icon. Régne Animal, Ins. p. 502 (1843) (Glaucopis). - Druce, Biol. Centr.-Am., Het. I, p. 72 (1884); 2, p. 352 (1897). - Hamps., Cat. Lep. Phal. 1, p. 446, f. 240 (1898).

cruenta Clem., Proc. Acad. Philad. p. 545 (1860). haematodes Boisd., Lép. Guatem. p. 82 (1870) (Charidea).

ab. gloriosa Walk., List Lep. Ins. Br. Mus. I, p. 215 (1854) (Euchromia). - Butl., Mlustr. Het. Br. Mus. 1, p. 43 , t. 10, f. 10 (1877). Hamps., Cat. Lep. Phal. 1, p. 446 (1898).

Bolivia

Colombia, Venezuela, Ecuador, Peru

Colombia

Mexico,

Brit.-

Honduras, Guatemala, Costarica, Panama, Venezuela

Costarica, Panama

\section{Amazonas}

Mexico,

Brit.-

Honduras, Guatemala 
ab. bivulnerata Grote \& Rob., Ann. Lyc. Nat. Hist. Netw York 8, p. 365, t. 13, f. 2 (1867) (Charidea). — Hamps., Cat. Lcp. Phal. 1, p. 446 (1898).

ab. eximia Boisd., Lép. Guatem. p. 82 (1870) (Charidea). - Hamps., Cat. Lep. Phal. 1, p. 446 (1898).

Biologie: Hamps., Cat. Lep. Phal. 1, p. 446 (1898).

bertha Druce, Proc. Zool. Soc. Lond. p. 381, t. 40, f. 1 (1883) (Charidea). — Hamps., Cat. Lep. Phal. 1, p. 449 (1898).

Buckleyi Druce, Proc. Zool. Soc. Lond. p. 381, t. 40, f. 4 (1883) (Charidea). - Hamps., Cat. Lep. Phal. 1, p. 450 (1898).

chelidon Druce, Proc. Zool. Soc. Lond. p. 255, t. 19, f. 3 (1893) (Netastatia). - Hamps., Cat. Lep. Phal. 1, p. 450 (1898).

chloe Druce, Proc. Zool. Soc. Lond. p. 381 (1883) (Charidea). - Hamps., Cat. Lep. Phal. 1, p. 450 , t. 15, f. 1 (1898).

eilctipennis Walk., List Lep. Ins. Br. Mus. 31, p. 97 (1864) (Charidea). - Hamps., Cat. Lep. Phal. 1, p. 442 , t. 15 , f. 5 (1898).

azetas Druce, Ann. \& Mag. Nat. Hist. (6) 18, p. 35 (1896) (Charidea).

fastuosu Walk., List Lep. Ins. Br. Mus. 1, p. 215 (1854) (Euchromia). - Hamps., Cat. Lep. Phal. 1, p. 443 (1898).

fuigida Herr.-Schäff., Ausscrcur. Schmett. p. 74 , f. 235 (1851) (Charidea).

Girardi Dogn., Ann. Soc. Ent. Bclg. 46, p. 229 (1902) glancopoides Walk., List Lep. Ins. Br. Mus. 2, p. 325 (180̄4) (Dioptis). - Hamps., Cat. Lep. Phal. 1, p. 451 , t. 15 , f. 11 (1898).

griseldis Druce, Biol. Centr.-Am., Het. 1, p. 72, t. 8, f. 21 (1884) (Charidea). - Hamps., Cat. Lcp. Phal. 1, p. 445 (1898).

liuramn Butl., Journ. Linn. Soc. Lond., Zool. 12, p. 416 (1876) (Charidea); Ilustr. Het. Br. Mus. 1, p. 42, t. 18, f. 8 (1877). - Hamps., Cat. Lep. Phal. 1, p. 448 (1898).

ab. subgloriosa Stgr. Iris 7, p. 83 (1894) (Charidea). - Hamps., Cat. Lep. Phal. 1, p. 448 (1898).

Iyaloptera Dogn., Hét. nouv. Am. Sud 1, p. 4 (1910).

imperialis Druce, Proc. Zool. Soc. Lond. p. 380, t. 40, f. 2 (1883) (Charidea). - Hamps., Cat. Lcp. Phal. 1, p. 447 (1898).

julia Druce, Proc. Zool. Soc. Lond. p. 381 (1883) (Charidea). - Hamps., Cat. Lcp. Plial. 1, p. 447, t. 15 , f. 25 (1898).

Biologie: Schrottky, Iris 24, p. 153 (1910).

jueunda Walk., List Lep. Ins. Br. Mus. 2, p. 216 (1854) (Euchromia). - Burm., Descr. Phys. Rep. Argent. 5, p. 383 (1878). - Hamps., Cat. Iep. Phal. 1, P. 445 (1898).

Ecuador

Ecuador, Bolivia

Colombia, Ob.-

Amazonas

Ecuador

Colombia, Venezuela (M. C. Vind.)

Jamaica, Venezuela, São Paulo (M.C.Vind.)

Argentinien

Venezuela.

Pará

Mexico

Amazonas, Ecuador, Bolivia

Colombia

Ecuador

Peru,

S̃̃o Paulo,

Paraguay

Amazonas (M. C. Vind.), São Paulo (M.C.Vind.), 
fastuosa Mén., Cat. Lép. Pétersb. 2, p. 143, t. 14, f. 8 (1857) (Charidea) (nec Walk.).

lystra Drucc, Ann. \& Mag. Nat. Hist. (6) 18, p. 35 (1896) (Charidea). - Hamps., Cat. Lep. Phal. 1, p. 441, t. 15, f. 23 (1898).

masia Dogn., Le Nat. 11, p. 58 (1889) (Euagra); Lép. Loja p. 52, t. 4, f. 18 (1891). — Hamps., Cat. Lep. Phal. 1, p. 451 (1898).

melinda Dyar, Journ. Ncw York Lnt. Soc. 7, p. 175 (1899).

micans Herr.-Schäff., Aussercur. Schmett. p. 74, f. 233 (1854) (Charidea). - Hanps., Cat. Lep. Phal. 1, p. 443 (1898).

ab. fulgens Herr.-Schiiff., Aussercur. Schmett. p. 74, f. 234 (1854) (Charidea). - Hamps., Cat. Lep. Phal. 1, p. 442 (1898) (f. princip.).

obseura Druce, Ann. \& Mag. Nat. Hist. (7) 1, p. 406 (1898). - Hamps., Cat. Lep. Phal. 1, p. 449, t. 15 , f. 20 (1898).

orbona Druce, Proc. Zool. Soc. Iond. p. 283 , t. 19, f. 2 (1893) (Charidea). - Hamps., Cat. Lep. Phal. 1, p. 447 (1898).

panamensis Druce, Biol. Centr.-Am., Het. 1, p. 71 (1884) (Charidea). - Hamps., Cat. Lep. Phal. 1, p. 441, t. 15, f. 4 (1898).

perilla Druce, Proc. Zool. Soc. Lond. p. 381 (1883) (Charidea). - Hamps., Cat. Lep. Phal. 1, p. 449, t. 15 , f. 8 (1898).

perspicua Schaus, Proc. U. S. Nat. Mus. 29, p. 189 (1905). phocnicia Hamps., Cat. Lcp. Phal. 1, p. 447, t. 15, f. 6 (1898).

? pretiosa Burm., Descr. Phys. Rep. Argent, Atlas p. 59 (1880) (Charidea). - Hamps., Cat. Lcp. Phal. 1, p. 448 (1898) (huius generis?).

quadricolor Feld., Reise Nov., Lep. 2, p. 3, t. 102, f. 15 (1869). - Hamps., Cat. Lep. Phal. 1, p. 441 (1898).

Ribbei Druce, Biol. Centr.-Am., Hct. 1, p. 153, t. 14 f. 14 (1885) (Euagra). - Hamps., Cat. Lep. Phal. 1, p. 451 (1898).

samarea Schaus, Trans. Am. Ent. Soc. 30, p. 135 (1904). scintillans Butl, Cist. Ent. 1, p. 87 (1872) (Euchromia); Lep. Exot. p. 175, t. 61, f. 16 (1874). - Druce, Biol. Centr.-Am., Het. 1, p. 71 (1884). - Hamps., Cat. Lep. Phal. 1, p. 440 (1898).

submacula Walk, List Jop. Ins. Br. Mus. 1, p. 214 (1854) (Euchromia). - Butl., Illustr. Het. Br. Mus. 1, p. 41 , t. 13, f. 7 (1877). - Druce, Biol. Centr.-Am., Het. 1, p. 70 (1884); 2, p. 352 (1897). - Hamps., Cat. Lep. Phal. 1, p. 444 (1898).

Espiritu

Santo,

Rio Janeiro,

Santa

Catharina,

Paraguay,

Uruguay,

Argentinien

Colombia

Ecuador

Rio Janeiro

Colombia,

Venczuela

Peru

Santa

Catharina,

Argentinien

Panama

Ecuador

Bolivia

"

Argentinien

Colombia

Panama

Bolivia

Costarica,

Panama

Mexico,

Guatemala,

Costarica,

Panama,

Trinidad,

Venezuela,

Colombia

(M.C.Vind.) 
xenodice Druce, Biol. Centr.-An., Hct. 1, p. 72, t. 8,

f. 18,19 (1881) (Charidea). - Hamps., Cat. Lep. 37

Phal. 1, p. 444 (1898).

Costarica

Desmotricha Hamps.

Hamps., Ann. \& Mag. Nat. Hist. (8) 8, p. 396 (1911).

Trichodesma Hamps., Cat. Lep. Phal. 1, p. 451 (1898) (nec Lecontc 1861).

albicineta Hamps., Ann. \& M Iag. Nat. Hist. (7) 15, p. 430 (1905) (Trichodesma).

aurimacula Sehaus, Proe. U. S. Nat. Mas. 29, p. 190 (1905) ('Trichodesma).

imitata Druce, Proc. Zool. Soc. Lond. p. 382 (1883) (Diabaena ). - Hamps., Cat. Lep. Phal. 1, p. 452, f. 242 (1898).

obliquia Schaus, Journ. New York Ent. Soe. 6, p. 140 (1898) (Trichodesma). - Hamps., Cat. Lep. Phal. 1, p. 453, t. 15, f. 18 (1898).

oeeola Dyar, Proc. U. S. Nat. Mus. 38, p. 230 (1910) (Trichodesma).

trita Dugn., Hét. no uv. Am. Sud 3, p. 5 (1911) (Trichodesma).

uniformis Hamps., Cat. Lep. Plial. 1, p. 453, t. 15, f. 19 (1898) (Trichodesma).

ursula Cram., Pap. Exot. 4, p. 107, t. 345, f. E (1782) (Noctua). - Möselll,, Verh. zool.-bot. Ges. Wicn 27, p. 647 (1877). - Druce, Biol. Centr. Am., Het. 2, p. 360 (1897). - Hamps., Cat. Lep. Phal. 1, p. 452, f. 241 (1898).

Venczuela

"

Eeuador

São Paulo (M.

C. Vind.),

Rio Janeiro

Mexico,

Venezucla

Colombia,

Ecuador

Rio Janeiro

Costarica,

Surinam,

Pará,

Amazonas,

Santa

Catharina

(M.C.Vind.)

8

\section{Coreura Walk.}

Walk., List Lep. Ins. Br. Mlus. 31, p. 142 (1864). - Hamps., Cat. Lep. Phal. 1, p. 453 (1898).

Pseudocharidea Druec, Ann. \& Mag. Nat. Hist. (6) 18, p. 36 (1896); Biol. Centr.-Am., Hot. 2, p. 353 (1897).

Adamsi La'hy, The Ent. 32, p. 119 (1899).

eiou Druce, Ann. \& Hag. Nat. Hist. (6) 18, p. 36 (1896).

(Pseudocharidea); Biol. Centr.-Am., Het. 2, p. 353,

t. 72, f. 26 (1897). - Hamps., Cat. Lep. Phal. 1, p. 454 (1898).

euchromioides Walk., List Lop. Ins. Br. Mus. 31, p. 142 , (1864). - Hamps., Cat. Lep. Phal. 1, p. 453, f. 243 (1898).

fida Hübn., Zutr. Exot. Schmett. 3, p. 15, f. 445, 446, (1827) (Calodesma). - Grünberg, Berl. Ent. Zeitschr. 52, p. 66 (1907).

a tavia Hamps., Cat. Lep. Plaal, 1, p. 454, f. 244 (1898).

\author{
Ecuador \\ Costariea, \\ Panama \\ Colombia, \\ Venezucla \\ (M.C.Vind.) \\ Mexico (M. \\ C. Vind.) \\ Espiritu \\ Santo, \\ RioJaneiro, \\ Santa \\ Catharina \\ (M.C.Vind.)
}


Hampsoni Dogn., Ann. Soe. Ent. Belg. 42, p. 212 (1898).

interposita Hampi., Ann. \& Mag. Nat. Hist. (7) 8, p. 173 (1901).

lysimachides Druce, Ann. \& Mag. Nat. Hist. (6) 20 , p. 304 (1897). - Hamps., Cat. Lep. Phal. 1, 7

p. 454 , t. 15 , f. 14 (1898)

Ecuador

Venezuela

Teuarior, Peru

\section{Aclytia Hübn.}

Hübn., Verz. bek. Sehmett. p. 123 (1826). - Butl., Journ. Linn. Soc. Lond., Zool. 12, p. 414, t. 29, f. 17 (1876). - Hamps., Cat. Lep. Pha!. 1, p. 455 (1898).

Percote Walk., List Lep. Ins. Br. Mis. 2, p. 492 (1854).

albistriga Schaus, Ann. \& Mag. Nat. Hist. (8) 6, p. 191 (1910); (8) 7, p. 181 (1911).

bractea Möschl., Verh. zool.-bot. Ges. Wien 27, p. 645, t. 8, f. 15 (1877) (Sciopsyche). - Hamps., Cat. Lcp. Phal. 1, p. 458 (1898).

flavigutta Walk., List Lep. Ins. Br. Mns. 1, p. 246 (1854) (Euchromia). - Butl., Illustr. Het. Br. Mus. 1, p. 41 , t. 8, f. $3(1877)$. - Hamps., Cat. Lcp. Phal. 1, p. 456 (1898).

simulatrix Walk., List Lep. Ins. Br. Mus. 31, p. 106 (1864) (Pelochyta).

gynaecomorpln (gynamorpha) Hamps., Cat. Lep. Phal. 1, p. 457 (1898).

flavigutta Butl., Illustr. Het. Br. Mus. 1, p. 41, t. 8 , f. 3 (1877) (nec Walk.).

heber Cram., Pap. Exot. 3, p. 169, t. 287, f. A (1780) $(\operatorname{Sphin} x)$. - Druce, Biol. Centr.-Am., Het. 1, p. 69 (1884); 2, p. 352 (1897). - Hamps., Cat. Lep. Phal. 1, p. 457, f. 245 (18s8).

halys Crain., Pap. Exot. 4, p. 129, t. 357, f. C (1782) (Sphinx). - Burm. Deser. Phys. Rep. Argent. 5, 13. 385 (1878). - Bntl., Illustr. Het. Br. Mus. 1, p. 41 , t. 10 , f. 6 (1877).

flaviventris Möschl., Stett. Lint. Zeitg. 33, p. 349 (1872).

Biologie: Stoll, Suppl. Cram. p. 48, t. 10, f. 2,3 (1790).

mariamne Druce, Proc. Zool. Soc. Lond. p. 519, t. 32 , f. 3 (1885) (Charidea). - Hamps., Cat. Lcp. Phal. 1, p. 456 (1898).

petra Schaus, Proc. Zool. Soc. Lond. p. 276 (1892); Am. Am. Lep. p. 6, t. 1, f. 17 (1892). — Hamps., Cat. Lep. Phal. 1, p. 457 (1898).

punctata Butl., Journ. Linn. Soc. I.ond., Zool. 12, p. 414 (1876). - Druce, Biol. Centr.-Awi., Ilct. 1, p. 70 (1884); 2, p. 352 (1897). - Hamps., Cat. Icp. Phal. 1, p. 456 , t. 15 , f. 12 (1898).

heber Walk., List Lep. Ins. Br. Mus. 1, p. 244 (1854) (Euchromia) (nec Cram.).

pydna Druee, Am. \& Nag. Nat. Hist. (7) 3, p. 231 (1899).

Costarica

Surinam

São Paulo

Amazonas, Pará

Cuba,

Trinidad,

Hexico,

Guatemala,

Brit.-

Honduras,

Honduras,

Costarica,

Surinam,

Amazonas

(M.C. Vind.),

Pará,

Rio Janciro,

Paraguay,

Argentimien

Colombia

Pern

Brit.-

Honduras, Guatemala, Costarica, Pará

Eonador 
signatura Walk., List. Lep. Ins. Br. Mus. 2, p. 493 (1854) (Percoie). - Hamps., Cat. Lep. Phal. 1, p. 450, t. 15, f. 16 (1898).

terra Schaus, Journ. Now York Ent. Soc. 4, p. 133 (1896). - Hamps., Cat. Lep. Phal. 1, p. 458, t. 15 , f. 17 (1898).

ventralis Guér., Icon. Régne Animal, Ins. p. 503 (1843) (Glaucopis). - Druce, Biol. Centr.-Am., Het. 1, p. 70 (1884). - Hamps., Cat. Lep. Plial. 1, p. 458 (1898).

conspicua Druce, Biol. Contr.-Am., Het. 1, p. 70.

t. 8 , f. $23(1884)$; 2. p. $352(1897)$.

lucania Schaus, Ént. Am. 5, p. 89 (1889) (Glaucopis).

12

Westindien (ubi?)

São Paulo, Paraguay

Mexico, Guatemala, Costarica, Panama

\section{Procalypta Butl.}

Butl., Journ. Linn. Soc. Lond., Zool. 12, p. 411 (1876). - Hamps., Cat. Lep. Phal. 1, p. 459 (1898).

subeyanea Walk., List Iop. Ins. Br. Mus. 1, p. 230 (1854) (Euchromia). - Butl., Illustr. Het. Br. Mus. 1, p. 40, t. 8, f. 11 (1877). - Druce, Biol. Centr.-Am., Fict. 1, p. 67 (1884); 2, p. 351 (1897).

- Hamps., Cat. Lcp. Phal. 1, p. 459, f. 246 (1898). r. victorina Drucc, Biol. Centr.-Am., Het. 1, p. 67 , t. 8, f. 16 (1884). - Preiss, Abb. Nachtschw. p. 7 , t. 9, f. 6 (1888). - Hamps., Cat. Lep. 1 Phal. 1, p. 460 (1898).

Mexico

Costarica, Panama, Amazonas (M.C.Vind.)

\section{Uraga Walk.}

Walk., List Lcp. Ins. Br. Mus. 2, p. 464 (1854). - Hamps., Cat. Lep. Phal. 1, p. 460 (1898).

haemorrhoa Walk., List Lep. Ins. Br. Mus. 2, p. 465 (1854). - Hamps., Cat. Lep. Phal. 1, p. 460, f. 247 (1898).

rubrieollis Hamps., Ann. \& Mag. Nat. Hist. (7) 8, p, 173 (1901).

trilidn Dogn., Ann. Soc. Ent. Belg. 52, p. 153 (1908). Peru 3

\section{Gaugamela Walk.}

(Gangamela) Walk., List Lep. Ins. Br. Mus. 31, p. 140 (1864). - Hamps., Cat. Lep. Phal. 1, p. 460 (1803).

U xela Walk., List Lep. Ins. Br. Mus. 35, p. 1982 (1866).

ira Druce, Ann. \& Mag. Nat. Hist. (6) 18, p. 34 (1896) Panama

(Charidea); Biol. Centr.-An., Het. 2, p. 353, t. 72, f. 15 (1897). - Hamps., Cat. Lep. Phal. 1, p. 461 (1898).

saturnta Walk., List Lep. Ins. 13r. Mus. 31, p. 140 (1864). Brasilien 


\section{Euagra Walk.}

Walk., List Lep. Ins. Br. Mus. 2, p. 323 (1854). - Hamps., Cat. Lep. Phal. 1, p. 461 (1898).

Nepe Walk., List Lep. Ins. Br. Mus. 2, p. 336 (1854).

Callagra Butl., Cist. Ent. 2, p. 111 (1876).

angeliea Butl., Cist. Ent. 2, p. 110 (1876). - Hamps., Cat. Lep. Plial. 1, p. 463, t. 15, f. 26 (1898).

azurea Walk., List Lep. Ins. Br. Mus. 2, p. 325 (1854) (Dioptis). - Hanzss., Cat. Lep. Phal. 1, p. 464, t. 16, f. 2 (1898).

eaelestina Cram., Pap. Exot. 4, p. 107, t. 345, f. 9 (17S2) (Bombyx). - Hamps., Cat. Lcp. Phal. 1, p. 462 , f. 249 (1898).

interclusa Walk., List Lcp. Ins. Br. Mus. 2, p. 328 (1854) (Dioptis).

Biologie: Stoll, Suppl. p. 101, t. 21, f. 2 (1790).

eaernlea (caerula) Dogn., Lo Nat. 13, p. 125 (1891); Lép. Loja p. 83, t. 8, f. 5 (1894). - Hamps., Cat. Lep. Phal. 1, p. 464 (1898).

cerymica Druce, Proc. Zool. Soc. Lond. p. 284 (1893). - Hamps., Cat. Lep. Phal. 1, p. 462, t. 15, f. 7 (1898).

chien Hamps., Cat. Lep. Phal. 1, p. 463, t. 16, f. 1 (1898).

delectans Schaus, Ann. \& Mag. Nat. Hist. (8) 7, p. 612 (1911).

fenestra Walk., List Lep. Ins. Br. Mus. 2, p. 337 (1854) (Dioptis). - Hamps., Cat. Lep. Phal. 1, p. 465, t. 16, f. 7 (1898).

ha emantlhus Walk., List Lep. Ins. Br. Mus. 2, p. 324 (1854) (Dioptis). - Drucc, Biol. Centr.-Am., Het. 1, p. 81 (1884). - Hamps., Cat. Lep. Phal. 1, p. 463, f. 250 (1898).

intereisa Butl., Cist. Ent. 2, p. 111 (1876). - Druce, Biol. Centr.-Am., Het. 2, p. 358 (1897). Hamps., Cat. Lep. Phal. 1, p. 464, t. 16, f. 8 (1898).

a uxo Walk., List Lep. Ins. Br. Mus. 2, p. 327 (1854) (Dioptis) (ncc Hübn.).

latera Druce, Proc. Zool. Soc. Lond. p. 496, t. 42, f. 7 (1890) (Erchia). - Hamps., Cat. Lep. Phal. 1, p. 465 (1898).

splendida Butl., Cist. Ent. 2, p. 112 (1876) (Callagra). - Hamps., Cat. I.cp. Phal. 1, p. 464, t. 16, f. 3 (189S).

Colombia

Brasilien (ubi?)

Surinam, Pará

Ecuador

Panama

Peru (M. C. Vind.), Bolivia

Costarica

Brasilien (ubi?)

Mexico, Guatemala, Costarica, Panama

Panama, Venezuela, Bolivia 12

Ecuador, São Paulo

Santa Catharina (M.C.Vind.)

\section{Mydromera Butl.}

Butl., Cist. Ent. 2, p. 112 (1876). - Hamps., Cat. Lep. Phal. 1, p. 465 (1898). istlumia Feld., Reise Nov., Lep. 2, p. 4, t. 105, f. 24 (1868) Guatemala, (Euagra). - Druce, Biol. Centr.-Am., Het. 1, Brit.p. 81 (1884); 2, p. 359 (1897).

Honduras, 
notochloris Boisd., Lép. Guatem. p. 87 (1870)

Nicaragua (Euagra). - Hamps., Cat. Lep. Phal. 1, p. 466, f. 251 (1898).

Nolckeni Strlgr., Verh. zool.-bot. Ges. Wien 25, 1 1. 121 (1875) (Agyrta).

\section{Micragra Hamps.}

Hamps., Cat. Lop. Phal. 1, p. 466 (1988). vehrea Hamps., Ann. \& Mag. Nat. Hist. (7) 8, p. 174 (1901). sanguiceps Hamps., Cat. Lep. Phal. 1, p. 466, f. 252 (1898).

2

liio Grande do Sul

Brasilion (Iguarasuseo)

\section{Mevania Walk.}

Walk., List Lep. Ins. Br. Mus. 2, p. 442 (1854). - Hamps., Cat. Lep. Phal. 1, p. 467 (1898).

quadrieolor Walk., List Lep. Ins. Br. MLus. 2, p. 443 Venezuela, (1854). - Hamps., Cat. Lep. Phal. 1, p. 467 Eeuador 1 f. 253 (1898).

\section{Microgiton Feld.}

Feld., Reiso Nov., Lep. Atlas p. 6 (1869). - Hamps., Cat. Lep. Phal. 1, p. 467 (1898).

submacula Walk., List Lop. Ins. Br. Mus. 2, p. 472 (1854) (Virbia). - Hamps., Cat. Lep. Phal. 1, p. 467, f. 254 (1898).

selene Feld., Reise Nov., Lep. 2, p. 6, t. 103, f. 17 1 (1869).

Venezuela,

Colombia

(M.C.Vincl.),

Amazonas

(M.C.Vind.)

\section{Hyperphara Hamps.}

Hamps., Cat. Lep. Phal. 1, p. 468 (1898).

junctura Walk., List Lep. Ins. Br. Mus. 31, p. 211 (1864) Colombia (Melanchroia). - Hamps., Cat. Lep. Phal. 1, p. 468 , f. 255 (1898).

cingulosus Feld., Reise Not. Lep. 2, p. 6, t. 103, 1

f. 18 (1869) (Microgiton).

\section{Parastatia Hamps.}

Hamps., Cat. Lep. Phal. 1, p. 468 (1898).

parnassia Möschl., Verh. zool.-bot. Ges. Wien 27, p. 656,

Surinam

t. 8, f. 5 (1877) (Marissa). - Hamps., Cat. Lep.

Phal. 1, p. 468, f. 256 (1898). 


\section{Agyrta Hübn.}

Hübn., Verz. bek. Sehmett. p. 177 (1826). - Hamps., Cat. Lep. Phal. 1, p. 469 (1898).

Corydalla Walk., Iist Lep. Ins. Br. Mus. 7, p. 1650 (1857).

albisparsa Hamps., Cat. Lep. Phal. 1, p. 472, t. 16, f. 20 (1898).

auxo L., Syst. Nat. ed. 12, p. 805 (1767) (Sphinx). - Hübn., Samml. Exot. Selhmett. 1, t. 185 (1827). - Hamps., Cat. Lep. Phal. 1, p. 471 (1898).

chena Druce, Proe. Zool. Soe. Lond. p. 281 (1893). Hamps., Cat. Lep. Phal. 1, p. 470 , t. 16, f. 19 (1898).

conspicua Sehaus, Ann. \& Mag. Nat. Hist. (8) 7, p. 181 (1911).

dux Walk., List Lcp. Ins. Br. Mus. 2, p. 327 (1854) (Dioptis). - Hamps., Cat. Lep. Phal. 1, p. 469, f. 257 (1898).

aestiva Butl., Cist. Ent. 2, p. 113 (1870). (Druec, Biol. Centr.-Am., Het. 2, p. 332 (1896). phylla Druce, Proc. Zool. Soc. Lond. p. 282 (1893).

ab. superba Druce, Biol. Centr.-Am., Het. 1, p. 115, t. 12, f. 5 (1884); 2, p. 388 (1897) (Isostola). - Hamps., Cat. Lcp. Phal. 1, p. 470 (1898).

flavitincta Hamps., Cat. Lcp. Phal. 1, p. 471, t. 16, f. 21 (1898).

micilia Cram., Pap. Exot. 3, p. 62, t. 228, f. G (1780) (Bombyx). - Druce, Biol. Centr.-Am., Het. 2, p. 332 (1896). - Hamps., Cat. Lop. Phal. 1, p. 470 (1898).

Biologie: Stoll, Suppl. Cram. p. 101, t. 21, f. 3 (1790).

monoplaga Druce, Ann. \& Mag. Nat. Hist. (7) 1, p. 208 (1898). - Hanps., Cat. Lop. Phal. 1, p. 471, t. 16 , f. 25 (1898).

pandemin Druce, Proc. Zool. Soe. Jond. p. 282 (1893). - Hamps., Cat. Iop. Phal. 1, p. 472, t. 16, f. 26 (1898).

porphyria Cram., Pap. Exot. 4, p. 227, t. 397, f. E (1782) (Bombyx). - Möschl., Verh. zool.-bot. Ges. 27, p. 642 (1877). - Hamps., Cat. Lep. Phal. 1, p. 472 (1898).

cryptoleuca Walk., List Lep. Ins. Br. Mus. 7, p. 1650 (1856) (Corydalla).

varuna Druce, Ann. \& Mag. Nat. Hist. (7) 19, p. 299 (1907).

vitrea Sehaus, Ann. \& Mag. Nat. Hist. (8) 6, p. 191 (1910).

Santa

Catliarina

Venezuela,

Surinam,

Pará

Ob.-Ama-

zonas,

Bolivia

Costarica

Santa

Lueia,

Honduras,

Guatemala,

Panama,

Venezucla

Bolivia

Panama,

Venezuela,

(II.C.Vind.),

Surinam,

Franz.-

Guayana,

(M.C.Vind.)

Pará,

Eenador

Eeuador

Pará

Venezuela,

Surinam,

Franz.-

Guayana,

(M.C.Vind.),

Pará,

Amazonas

(M.C.Vind.),

Bolivia

Peru

Costariea 12 
Phara Walk.

Walk., List Lep. Ins. Br. Mus. 2, p. 321 (1854). - Hamps., Cat. Lep. Phal. 1, p. 473 (1898).

Olina Walk., Charact. Undoscr. Hot. p. 5 (1869).

flavieosta Herr.-Sehäff., Aussercur. Schmett. v. 74, f. 421 (1855) (Ecdemus). - Hamps., Cat. Lep. Phal. 1, p. 473, f. 258 (1898).

nyehthemeroides (nyctemeroides) Wall., Char. Undeser. Hot. p. 6 (1869) (Olina). - Hamps., Cat. Lep. Phal. 1, 1) 474 , t. 16, f. 4 (1898).

sanguipalpis Dogn., Ann. Soc. Ent. Belg. 5I, p. 224 (1907).

trivittata Walk., List. Lep. Ins. Br. Mus. 2, p. 322 (1854) (Dioptis). - Hawps., Cat. Lep. Phal. 1, p. 473,

4 f. $\left.259(1898){ }^{*}\right)$

Siao Paulo

(M.C.Vind.),

Rio Grando do Sul

Brasilicn

(ubi?)

Peru

(M.C.Vind.)

Patria?

\section{Ptychotrichus Schaus.}

(Ptychotrichos) Schaus, Proc. Zool. Soc. Lond. p. 227 (1894). - Hamps., Cat. Lep. Phal. 1, p. 477 (1898).

elongatus(-a) Schaus, Proc. U. S. Nat. Mus. 29, p. 190 (1905).

zeus Schaus, Proc. Zool. Soc. Lond. p. 228 (1894). - Venezuela 2 Hamps., Cat. Lep. Phal. 1, p. 477, f. 203 (1898).

Rio Janciro

\section{Delphyre Walk.}

Walk., List Lep. Ins. Br. Mus. 2, p. 537 (1854). - Hamps., Ann. \& MIag. Nat. Hist. (7) 15, p. $430(1905)$.

Neacerea Hamıs., Cat. Lep. Phal. 1, p. 478 (1898).

Nodoza Schaus, Journ. Now York Lint. Soc. 4, p. 150 (1890).

aelytioides Hamps., Ann. \& Mag. Nat. Hist. (7) 8, p. 174 Tranz.(1901).

albiventa(-us) Druce, Anu. \& Mag. Nat. Hist. (7) 1, p. 400 (1898) (Neacerea). - Hatsps., Cat. Lep. Phal. 1, p. 480 , t. 16, f. 22 (1898).

atara Druce, Biol. Centr.-Am., Het. 1, p. 74 (1884) (Heliura). - Hamps., Cat. Lcp. Phal. 1, p. 481, t. 16, f. $24(1898)$.

brunnea Druce, Ann. \& Mag. Nat. Hist. (7) 2, p. 220 (1898) (Neacerea ). - Hamps., Cat. Lep. Phal. 1, p. 478, f. $264(1898)$.

discalis Druce, Ann. \& Itag. Nat. Hist. (7) 15, p. 463 (1905) (Neacerea).

dizona Druce, Ann. \& Mag. Nat. Hist. (7) 1, p. 400 (1898) (Neacerea). - Hamps., Cat. Lep. Pial. 1, p. 481, t. 16 , f. $12(1898)$.

elegans Lathy, The Ent. 32, p. 119 (1899) (Neacerea). Guayana

Amazonas (M. C. Vind.),

Ninas Geračs, Rio Janciro

Panama

Esspiritu

Santo

Venezuela

Franz.-

Guayana

Ecuador flaviecps Druce, Ann. \& Nag. Nat. Hist. (7) 15, p. 463 Venezucla (1905) (Neacerea).

*) Genera Azatrephes Hmps., Antaxia Hmps., Gonotrephes Hmps., ut jam W. Rothschild iudieavit (Nov. Zool. 17, p. 1, 1810) potius ad Artitas referenda sunt. 
flaviventralis Hamps., Ann. \& Mag. Nat. Hist. (7) 8, Venczucla p. 174 (1901.)

liebes Walk., Tist Lep. Ins. Br. Mus. 2, p. 537 (1854). Honduras, - Hamps., Ann. \& Mag. Nat. Hist. (7) 15, São Paulo p. $430(1905)$.

tristis Schaus, Journ. New York Ent. Soc. 4, p. 150 (1896) (Nodoza).

macella Dogn., Hét. nouv. Am. Sud 2, p. 11 (1911) (Neacerea).

maculosa Hamps., Cat. Lep. Phal. 1, p. 479, f. 265 (1898) (Neacerea).

minuta(-min) Möschl., Verh. zool.-bot. Ges. Wien 27, p. 651, t. 9, f. 19 (1877) (Eucereon). - Hamps., Cat. Lep. Phal. 1, p. 479 (1898).

monotona Dyar, Proc. U. S. Nat. Mus. 38, p. 231 (1910).

nigra Schaus, Trans. Am. Ent. Soc. 30, p. 136 (190t) (Neacerea).

pusilla Butl., Trans. Ent. Soc. Lond. p. 44 (1878) (Acridopsis). - Hamps., Cat. Lep. Phal. 1, p. 480, t. 16 , f. 23 (1898).

? dares Cram., Pap. Exot. 1, p. 76, t. 48, f. F (1775) (Sphinx) (nomen prius, sed incertum).

pyroperas Hamps., Ann. \& Mag. Nat. Hist. (8) 8, p. 396 (1911).

pyrozona Druce, Ann. \& Mag. Nat. Hist. (7) 15, p. 463 (1905) (Neacerca).

rhodocrypta Druce, Ann. \& Mag. Nat. Hist. (7) 15, p. 463 (1905) (Neacerea).

ruseiceps Dogn., Ann. Soc. Ent. Belg. 53, p. 217 (1909).

rubricincta Hamps., Cat. Lep. Phal. 1, p. 479, t. 16, f. 5 (1898) (Neacerea).

ruliventris Schaus, Proc. Zool. Soc. Lond. p. 228 (1894) (Aclytia). - Druce, Biol. Centr.-Ann., Het. 2, p. 352 , t. 72 , f. 13 (1897). - Hamps., Cat. Lep. Phal. 1, p. 481, f. 266 (1898).

subapienlis Jones, Trans. Fint. Soc. Lond. p. 145 (1908). suffusa Lathy, The Ent. 32, p. 120 (1899) (Neacerea).

testacea Druee, Biol. Centr.-Am., Hct. 1, p. 74 (1884) (Heliura ). - Hamps., Cat. Lep. Phal. 1, p. 482, t. 16, f. 6 (1898).

tetilla Dogn., Ann. Soc. Ent. Belg. 42, p. 344 (1898) (Nercerea).

variaus Hamps., Ann. \& Mag. Nat. Hist. (7) 8, p. 174 Amazonas 27 (1901).

Franz. -

Pará

Guayana

Venezucla, Surinam

Bolivia

Mexico

Panama

Amazonas,

?Westindien

Sao Paulo (MI. C. Vind.), Rio Grande do Sul, Paraguay

Venezuela

Franz.-

Guayana

Panama (J.Taboga)

Mexico, Bolivia

Paraná

Brit.-

Guayana

Gitatemala, Panama

Ecuador

\section{Heliuma Butl.}

Butl., Journ. Linn. Soc. Lond., Zool. 12, p. 417, t. 29, f. 13, 17 (1876). Hamps., Cat. Lep. Phal. 1, p. 482 (1898).

balia (-um) Hamps., Cat. Lep. Phal. 1, p. 494, t. 16, f. 13 Honduras (1898) (Eucereon); Ann. \& Mag. Nat. Hist. (7) 15, p. 430 (1905). 
dolens Schaus, Proc. U. S. Nat. Mus. 29, p. 191 (1905). emerentia Dogn., Ann. Soc. Ent. Bclg. 42, p. 344 (1898). fulvipicta Kaye, Tho Ent. 44, p. 146 (1911).

hecale Schaus, Proc. Zool. Soc. Lond. p. 277 (1892) (Aclytia); Am. Lep. p. 6, t. 1, f. 18 (1892). Hamps., Cat. Lep. Phal. 1, p. 483 (1898).

phraeosoma Druce, Ann. B Mag. Nat. Hist. (7) 15, p. 464 (1905).

pierus Cram., Pap. Exot. 4, p. 148, t. 367, f. I (1782) (Sphinx). - Hamps., Cat. Lep. Phal. 1, p. 484 (1898).

lucis Butl., Trans. Ent. Soc. Lond. p. 45 (1878) (Acridopsis).

rhodophila Walk., List Lop. Ins. Br. Mus. 7, p. 1638 (1856) (Eucerea). - Hamps., Cat. Lep. Phal. 1, p. 483, f. 267 (1898).

tetragramma var. Walk., List Lep. Ins. Br. Mus. 1, p. 268 (1854) (Euchromia). - Druce, Biol. Centr.-Am., Het. 1, p. 86 (1884).

pyrrhosoma Butl., Journ. Linn. Soc. Lond., Zool. 12, p. 418 (1876). - Druce, Biol. Centr.Am., Het. 2, p. 354, t. 72, f. 19 (1897).

solicauda Butl., Journ. Linn. Soc. Isond., Zool. 12, p. 418 (1876); Illustr. Het. Br. Mus. 1, p. 44, t. 9, f. 4 (1877). - Dreue, Biol. Centr.-Am., Het. 1, p. 74 (1884).

sanguipalpia Hamps., Cat. Lep. Phal. I, p. 484, f. 268 (1898).

tetragramma Walk., I.ist Lep. Ins. Br. Mus. 1, p. 268 (1854) (Euchromia). - Hamps., Cat. Lep. Phal. 1, p. 482 , t. 16 , f. 9 (1898).

thisbe (thysbe) Möschl., Verh. zool.-bot. Ges. 27, p. 643, t. 8, f. 14 (1877) (Acridopsis). - Hamps., Cat. Lop. Phal. 1, p. 484 (1898).

umbrimacula Schaus, Proc. U. S. Nat. Mus. 29, p. 191 (1905).

zonata Druce, Ann. \& Mag. Nat. Hist. (7) 15, p. 464 (1905).

Venezucla

Ecuador

Brit. -

Guayana

Rio Janeiro

Venezucla

Surinam, Amazonas

Honduras,

Costarica,

Surinam,

Pará

Santa

Catharina

Pará

Surinam

Franz.-

Guayana 13

\section{Encereum Hübn.}

(Eucereon) Hübn., Verz. bek. Schmett. p. 123 (1826). - Hamps., Cat. Lep. Phal. 1, p. 485 (1898.)

Erithales Pocy, Cent. Lep. Cuba p. 16, 1832).

Theages Walk., List Lep. Ins. Br. Mus. 3, p. 721 (1855).

Acrido psis Butl., Journ. Linn. Soc. Lond., Zool. 12, p. 418 , t. 29, f. 14 (1876). Hlöschl., Verh. zool.-bot. Ges. Wien 27, p. 643 (1877). Galethalea Butl., Journ. Linn. Soc. Jond., Zool. 12, p. 424 (1876).

abdominale(-is) Walk., List Lep. Ins. Br. Mus. 3, p. 731 (1855) (Carales). - Hamps., Cat. Lep. Phal. I, p. 509 , t. 17, f. 12 (1898).

aeolun Hamps., Cat. Lep. Phal. 1, p. 498, t. 16, f. $16(1898)$.

rhodophila Druec, Biol. Centr.-An., Het. 1, p. 86 (1884) (nec Walk).

aetolia Druce, Ann. \& Mag. Nat. Hist. (7) 5, p. 507 (1900). albinotum(-a) Dogn., Hét. nouv. Am. Sud 1, p. 5 (1910).

Venezuela

Mexico, Guatemala

Colombia 
album (-a) Druce, Ann. \& Mag. Nat. Hist. (6) 13, p. 172 (1894) (Idalus); Biol. Centr.-Am., Het. 2, p. 366, t. 73, f. 22 (1897). - Hamps., Cat. Lep. Phal. 1, p. 514 (1898).

amadis Schaus, Journ. New York Ent. Soc. 4, p. 133 (1896). - Druce, Biol. Cintr.-Am., Het. 2, p. 362 , t. 73, f. 10 (1897). - Hamps., Cat. Lep. Phal. 1, p. 503 (1898)

antonia Druce, Ann. \& Mag. Nat. Hist. (7) 18, p. 80 (1906).

aoris Môsschl., Verh. zool.-bot. Ges. Wien 27, p. 647, t. 8, f. 16 (1877). - Hamps., Cat. Lep. Phal. 1, p. 509 (1898).

alppunctatum (-a) Doga., Bull. Soc. Ent. France 60, p. CLXXV (1892); Lép. Loja p. 83, t. 8, f. \& (1894). - Hamps., Cat. Lep. Phal. 1, p. 491 (1898).

arehias Stoll, Suppl. Cram. p. 66, t. 14, f. 9, 10 (1790) (Sphinx). - Sepp, Surin. Vlind. 3, p. 271, t. 124, (1848). - IIamps., Cat. Lep. Phal. 1, p. 485, f. 269 (1898).

Biologie: Stoll, Suppl. Cram. p. 66, t. 14, f. $6-8$ (1790). - Sepp, Surin. Vlind. 3, p. 271, t. 124 (1848). - Hamps., Cat. Lep. Phal. 1, p. 485 (1898).

arenosum Buti., Illustr. Het. Br. Mus., 1 p. 51, t. 17, f. 10 (1877). - Druce, Biol. Centr.-Am., Het. 1, p. 87 (1884) (pr. p.); 2, p. 363 (1897). - Hamps., Cat. Lop. Pha!. 1, p. 492 (1898).

aroa Schaus, Proc. 'Zool. Soc. Lond, p. 229 (1894). Druce, Biol. Contr.-Am., Het. 2, n. 363 (1897). - Hamps., Cat. Lep. Phal. 1, p. 511, t. 17, f. 15 (1898). costulatum Druce, Biol. Centr.-Am., Het. 1, p. 87 (1884 (pr. p.)

atriguttum (-a) Druce, Ann. \& Mag. Nat. Hist. (7) 15 , p. 464 (1905).

baleris Dyar, Proc. U. S. Nat. Mus. 38, p. 232 (1910).

Birchelli Druce, Ann. \& Mag. Nat. Hist. (7) 7, p. 74 (1901).

carabayanum(-a) Schaus, Proc. U. S. Nat. Mus. 29, p. $191(1905)$.

casca Dogn., Ann. Soc. Ent. Belg. 38, p. 680 (1894). - Hamps., Cat. Lep. Phal. 1, p. 501, t. 17, f. 1 (1898).

chalcodon Druce, Proc. Zool. Soc. Lond. p. 287, t. 19, f. 6 (1893). - Hamps., Cat. Lep. Phal. 1, p. 496 (1898).

cimonis Schaus, Ann. \& Mag. Nat. Hist. (8) 6, p. 193 (1910).

cinctum Schaus, Journ. Now York Ent. Soc. 4, p. 134 (1896).

Clementsi Schaus, Am. Lep. p. 10, t. 1, f. 25 (1892). - Hamps., Cat. Lep. Phal. 1, p. 496 (1898).

coenobita Möschl.. Abl. Senekenb. Ges. 14, p. 20, f. 1 (1886) (Galethalea). - Hamps., Cat. Lep. Phal. 1, p. 505 (1898).
Mexice,

Fio Grande do Sul (MI.

C. Vind.)

Mexico,

Colombia

Poru

Surinam

Ecuador

Surinam, Amazonas

(M. C.

Vind.),

Südost-

Brasilien

(ubi?)

Mexico,

Guatemala,

Panama,

Amazonas,

Espiritu

Santo

Mexico

Guatemala,

Venezuela,

Santa

Catharina

(M.C.Vind.)

Venezuela

Mexico

Colombia

Peru

Écuador, Bolivia

Rio Janeiro, São Paulo

Costarica, Venezuela, Ecuador

Trinidad

Santa lucia

Jamaica 
eomplientum Butl., Illustr. Het. Br. Mius. 1, p. 50, t. 16, f. 12 (1877). - Hamps., Cat. Lep. Phal. 1, p. 503 (1898).

confine (-is) Herr.-Schäff., Aussereur. Schmett. p. 74, f. 277 (1855) (Charidea). - Druce, Biol. Centr.Am., Het. 1, p. 80 (1884); 2, p. 358 (1897). Hamps., Cat. Lep. Phal. 1, p. 508 (1898).

v. curolina Fy. Edw., Ent. Am. 2, p. 166 (1887) (Nelphe). - Dyar, Proc. U. S. Nat. Mus. 23, p. $262(1900)$.

confine Holl., Moth. Book p. 100, t. 13, f. 10 (1903).

Biologie: Dyar, Proc. U. S. Nat. Mus. 23, p. 262 $(1900)$

consortum (-a) Schaus, Ann. \& Mag. Nat. Hist. (8) 6, p. $192(1910)$.

costimotram Dogn., Amn. Soc. Ent. Belg. 44, p. 436 (1900).

costulatum (-a) Herr.-Schäff., Aussercur. Schmett. 1, p. 74, f. 276 (1855) (Charidea). - Druce, Biol. Centr.Am., Het. 1, p. 87 (1884) (pr. p.). - Hamps., Cat. Lep. Phal. 1, p. 510 (1898).

cubense (-is) Schaus, Trans. Am. Ent. Soc. 30, p. 136 (1904).

darantasia Drucc, Ann. \& Mag. Nat. Hist. (6) 15, p. 45 (1895).; Biol. Centr.-Am., Het. 2, p. 363, t. 73, f. 12 (1897). - Hamps., Cat. Lep. Phal. 1, p. 501 (1898).

Davidi Dogn., Le Nat. (2) 3, p. 11 (1889) (Galethalea); Lép. Loja p. 48, t. 4, f. 4 (1891). - Hamps., Cat. Lep. Phal. 1, p. 490 (1898).

decorum Schaus, Ann. \& Mag. Nat. Hist. (8) 6, p. 193 (1910).

diseolor Walk., List Lop. Ins. Br. Mus. 7, p. 1640 (1856). - Druce, Biol. Centr.-Am., Het. 2, p. 362 (1897). - Hamps., Cat. Le1). Phal. 1, p. 513 (1898).

rububa Druce, Ann. \& Mag. Nat. Hist. (6) 15 , p. 46 (1895); Biol. Centr.-Am., Het. 2, p. 364, t. 73 , f. 17 (1897).

dorsipunetum (-a) Hamps., Ann. \& Mag. Nat. Hist. (7) 15, p. 430 (1905).

erythrolepe (-is) Dyar, Proc. U. S. Nat. Mus. 38, p. 232 (1910).

excaratum(-a) Dogn., Hét. nouv. Am. Sud 1, p. 5 (1910). ab. flavum (-a) Dogn., Hét. nouv. Am. Sud 1, p. $6(1910)$.

fallum Druce, Ann. \& Mag. Nat. Hist. (7) 6, p. 65 (1900). flavieaput Hamps., Cat. Lep. Phal. 1, p. 501 (1898). leucophaea, Druce, Biol. Centr.-Am., Het. 1, 1) 84, t. 9 , f. 9 (1884); 2. p. 361 (1897) (Theages) (ncc. Walk.).

Ilavieinetum (-a) Schaus, Proc. U. S. Nat. Mus. 29, p. 192 (1905).
Amazonas

Mexico,

Guatemala,

Costarica,

Panama,

Venezuela,

Brasilien

(ubi?)

Florida

Costarica

Brasilien (ubi?).

Panama, Venezuela

Cuba

Costarica

Ecuador, Bolivia, Peru

Costarica

Mexico, Costarica,

Paulama, São Paulo,

Rio Janeiro.

Bolivia

Venezuela, Brasilien (ubi ?, M. C. Vind.), Paraguay

Mexico

Franz.Guayana

Venezucla

Mexico, Guatemala. Costarica, Ecuador Peru 
flavopunctatum Dogn., Hét. nouv. Am. Sud 2, p. 12 (1911).

formosum Dogn., Le Nat. 27, p. 9 (1905).

giganteum Druce, Ann. \& Mag. Nat. Hist. (7) 6, p. $6 \tilde{\text { I }}$ (1900).

guacolda Poey, Cent. Lep. Cuba p. 14 (1832) (Erithales). - Hamps., Cat. Lep. Phal. 1, p. 509 (1808) (pr.p.).

Hoegei Drucc, Biol. Centr.-Am., Het. 1, p. 86 (1884). - Hamps., Cat. Lep. Phal. I, p. 492, t. 16, f. I1 (1898).

hyalinum Kaye, Trans. Ent. Soc. Lond. p. 119, t. 5, f. 11 (1901).

ignotum (-a) Druce, Ann. \& Mag. Nat. Hist. (7) 18, p. $80(1906)$.

Imriei Druce, Proc. Zool. Soc. Lond. p. 322, t. 25, f. 6 (1884). - Hamps., Cat. Lep. Phal. I, p. 496 (1898).

inconspicu um Lathy, The Ent. 32, p. 120 (1899).

ino Druce, Ann. \& Mag. Nat. Hist. (7) 5, p. 508 (1900).

intranotatum Dogn., Ann. Soc. Ent. Belg. 44, p. 436 (1900).

irroratum (-a) Schaus, Trains. Am. Ent. Soc. 30, p. 136 (1904).

ladas Schaus, Proc. Zool. Soc. Lond. p. 278 (1892); Am. Lep. p. 10, t. 1, f. 26 (1592). - Hamps., Cat. Lep. Phal. 1, p. 510 (1898).

latifascium(-a) Walk., List Lep. Ins. Br. Mus. 7, p. 1639 (1856). - Druco, Biol. Centr.-Am., Het. 1, p. 85 (1881). - Hamps., Cat. Lop. Phal. 1, p. 498 , t. 16, f. 14 (1898).

Lemoulti Schaus, Proc. U. S. Nat. Mus. 29, p. 192 (1905).

leprotum (-a) Druce, Ann. \& Mag. Nat. Hist. (7) 15, p. 465 (1905).

Ieria Druce, Biol. Centr.-Am., Het. 1, p. 87, t. 9, f. 14 (1884); 2, p. 363 (1897). - Hamps., Cat. Lep. Phal. I, p. 493 (1898).

lerioides Schaus, Journ. New York Ent. Soc. 9, p. 44 (1901).

leucophacum (-a) Walk., List Lep. Ins. Br. Mrs. 3, p. 722 (1855) (Theages). - Hanips., Cat. Lep. Phal. 1, p. 502, t. 17, f. 2 (1898).

lineatum (-a) Dogn., Le Nat. 11, p. 173 (1889) (Theages); Lép. Loja p. 48, t. 4, f. 5 (1891). - Hamps., Cat. Lep. Phal. 1, p. 512 (1898).

lutetia Druce, Biol. Centr.-Am., Het. 1, p. 88, t. 9, f. 11 (1884). - Hamps., Cat. Lep. Phal. 1, p. 502 (1898).

Colombia

Peru

Colombia

Cuba

Mexico

Trinidad

Peru

Guadeloupe, Dominica

Brit.-

Guayana

Colombia

Ecuador

Cuba

Rio Janeiro

Mexico,

Guatemala,

Brit.-

Honduras,

Panama,

Trinidad,

Venezuela

Colombia,

(M.C.Vind.),

Pará, Peru

Franz.-

Guayana

Venezuela

Mexico, Panama, Ecuador, Bolivia

Mexico

Rio Janeiro, Paraguay

Ecuador, Bolivia

Panama 
maja Drucc, Biol. Centr.-Am., Hct. 1, p. 86, t. 9, f. 13 (1884); 2, p. 363 (1897). - Hamps., Cat. Lep. Phal. 1, p. 499 (1898).

mareatum(-a) Schaus, Journ. New York Ent. Soc. 9, p. 43 (1901).

marieum(-a) Cram., Pap. Exot. 1, p. 31, t. 20, f. F, G (1775) $(\operatorname{Sphin} x)$. - Butl., Illustr. Het. Br. Mus. 1, p. 45, t. 9, f. 6 (1876). - Hamps., Cat. Lep. Phal. 1, p. 490 (1898).

grylloides Walk., List Lep. Ins. Br. Mus. 1, p. 271 (1854) (Euchromia).

tralassica Feld., Reise Nov., Lop. 2, p. 4, t. 102 f. 18 (1869).

virescens Möschl., Verl. zool.-bot. Ges. Wien 27, p. 644 , t. 10 , f. 53 (1877) (Acridopsis).

marmoratun Butl., Illustr. Het. Br. Mus. 1, p. 50, t. 16. f. 4 (1877). - Hamps., Cat. Lep. Phal. 1, p. 502 (1898).

flavofasciatum Möschl., Verh. zool.-bot. Ges. Wien 27, p. 651, t. 9, f. 20 (1877).

Mathani Schaus, Journ. New York Ent. Soc. 9, p. 44 (1901).

melanoperas Hamps., Cat. Lep. Phal. 1, p. 495, t. 16, f. 10 (1898).

merula Dogn., Lo Nat. (2) 5, p. 278 (1891) (Theages); Lép. Loja p. 83, t. 8, f. 9 (1894). - Hamps., Cat. Lep. Phal. 1, p. 513 (1898).

meruloldes Schaus, Proc. U. S. Nat. Mus. 29, p. 192 (1905). metalobum(-a) Hamps., Ann. \& Mag. Nat. Hist. (8) 4, p. $348(1909)$.

metocdesis (metoidesis) Hamps., Ann. \& Mag. Nat. Hist. (7) 15, p. 430 (1905).

cinctum Hamps., Cat. Lep. Phal. 1, p. 486, f. 271 (1898) (nec Schaus).

latifascia Butl., Journ. Linn. Soc. Zool. 12, p. 419 (1876) (nec Walk.).

mintmm Druce, Biol. Centr.-Am., Het. 1, p. 87 (1884); 2, p. 363 , t. 73 , f. 7 (1897). - Hamps., Cat. Lep. Phal. 1, p. 485 , f. 270 (1898).

Drucci Kirby, Cat. Het. p. 201 (1892).

mizar Druce, Ann. \& Mag. Nat. Hist. (7) 3, p. 231 (1899). myrina Druce, Biol. Centr.-Am., Het. 1, p. 84, t. 9, f. 10 (1884); 2, p. 362 (1897). - Hamps., Cat. Lcp. Phal. 1, p. 508 (1898).

myrtusa Druce, Biol. Centr.-Am., Het. 1, p. 85 (1884); 2, p. 362, t. 73, f. 14 (1897). - Hamps., Cat. Lep. Phal. 1, p. 500 (1898).

nebulosimm Dogn., Lo Nat. 11, p. 58 (1889); Lép. Loja, p. 49 , t. 4, f. 6 (1891). - Hamps., Cat. Lep. Phal. 1, p. 513 (1898).

obscurum(-a) Möschl., Stctt. Ent. Zcitg. 33, p. 348 (1872) (Aclytia). - Druce, Biol. Centr.-Am., Het. 1, p. 80 (1884); 2, p. 358 (1897). - Hamps., Cat. Lep. Phal. 1, p. 490 (1898).
Mexico, Guatemala, Costarica, Panama, Ecuador, Trinidad, Surinam (M.C.Vind.)

Paraguay

Surinam, Franz.Guayana, Amazonas, Pará

\section{Surinam,}

Amazonas

Ecuador

Amazonas

Ecuador

Peru

"

Pará

Ecuador

Mexico, Guatemala

Mexico, Costarica

Ecuador

Mexico, Costarica, Panama, Surinam, 
stellifera Butl., Illustr. Het. Br. Mus. 1, p. 48, t. 16, f. 10 (1877) (Epanycles).

Amazonas, Paraguay ochrotum(-a) Hamps., Ann. \& Mag. Nat. Hist. (7) 15, p. 431 (1905).

guacolda Hamps., Cat. Lep. Phal. 1, p. 509 (1898) (pr. p.).

Ockendeni Druce, Ann. \& Mag. Nat. Hist. (7) 18, p. 80 (1906).

palladu Druce, Ann. \& Mlag. Nat. Hist. (7) 18, p. 80 (1906). papayanum Dogn., Ann. Soc. Ent. Belg. 46, p. 229 (1902) patrona Schaus, Journ. Now York Ent. Soc. 4, p. 134 (1896). - Druce, Biol. Centr.-Am., Het. 2, p. 364 , t. 73, f. 13 (1897). - Hamps., Cat. Lep. Phal. 1, p. 500 (1898).

patulum(-a) Dogn., Hét. nouv. Am. Sud 3, p. 5 (1911). perstriatum(-n) Hamps., Ann. \& Mag. Nat. Hist. (8) 4, p. 349 (1909).

phaeophlebium(-a) Hamps., Ann. \& Mag. Nat. Hist. (7) 19, p. 226 (1907).

phaeoproetum Hamps., Cat. Lcp. Phal. 1, p. 512, t. 17, f. $16(1898)$.

costulatum Druce, Biol. Centr.-Am., Het. 1, p. 87 (1884) (pr. p.).

pica Walk., List Lep. Ins. Br. Míus. 3, p. 743 (1855) (Halesidota). - Druce, Biol. Centr.-Ain., Het. 2, p. 358 (1897). - Hamps., Cat. Lep. Phal. 1, p. 506, t. 17 , f. 21 (1898).

v. tigratum (-a) Herr.-Schäff., Aussereur. Schmett. p. 74, f. 278 (1855) (Charidea). - Hamps., Cat. Lep. Phal. 1, p. 506 (1898).

peruviana Schaus, Proc. Zool. Soc. Iond. p. 277 (1892) (Galethalea); Am. Lep. p. 8, t. 1, f. 22 (1892).

pilati(-ii) Walk., List Lep. Ins. Br. Mus. 1, p. 270 (1854) (Euchromia). - Druce, Biol. Centr.-Am., Het. 1, p. 86 (1884); 2, p. 363 (1897). 一 Hamps., Cat. Lep. Phal. 1, p. 499 (1898).

sylvius Sepp, Surin. Vlind. 2, p. 149, t. 71, (1848) (nec Stoll) (Sericaria).

conjunctum Möschl., Verh. zool.-bot. Ges. Wien 27, p. 649, t. 8, f. 17 (1877).

Biologie: Sepp, Surin. Vlind. 2, p. 149, t. 71 (1848). - Hamps., Cat. Lep. Phal. 1, p. 497 (1898) (sylvius).

plumbieollum Hamps., Cat. Lep. Phal. 1, p. 512, t. 17, f. 20 (1895).

pometinum(-a) Druce, Ann. \& Mag. Nat. Hist. (6) 13, p. 172 (1894); Biol. Centr.-Am., Het. 2, p. 363, t. 73, f. 11 (1897). - Hamps., Cat. Lep. Phal. 1, p. 512 (1898).

pseudarehias Hamps., Cat. Lep. Phal. 1, p. 497, f. 272 (1898).

archias Druce, Biol. Centr.-Am., Het. 1, p. 85 (1884) (nec Stoll).

punetatum(-a) Guér., Icon. Régne Animal, Ins. p. 515 (1844) (Chelonia). - Hamps., Cat. Lep. Phal. 1, p. 494 (1898).

Peru

Paraná

Colombia

Mexico,

Venezuela

Peru

Peru

Argentinien

Guatemala, Costarica

Panama

Rio Janeiro, Szo Pauli, (M. C.

Vind.)

Costarica,

Panama,

Venezuela

(M.C.Vind.),

Peru,

Brasilien

(ubi?),

Argentinien

Mexico, Guatemala,

Honduras,

Colombia,

Surinam

Santa

Catharina

Panama

Mexico, Guatemala, Honduras, Amazonas

Guatemala, Surinam, Amazorias, 
mitigata Walk., List Lep. Ins. Br. Mus. 7, p. 1639 (1857).

cribrum Mïschl., Verh. zool.-bot. Ges. Wicn 27 , p. 648 (1877).

zamorae Dogn., Ann. Soc. Ent. Belg. 38, p. 681 (1894).

reticulatum Butl., Illustr. Het. Br. Mus. 1, p. 50, t. 17 , f. 9 (1877).

arenosum Druce, Biol. Centr.-Am., Het. 1, p. 87 (1884) (pr. p.) (nec Butl.).

pyrozonum(-a) Hamps., Ann. \& Mlag. Nat. Hist. (8) 8, p. 396 (1911).

quadrieolor Walk., List Lep. Ins. Br. Mus. 3, p. 722 (1855) (Theages). - Druce, Biol. Centr.-Am., Hct. 1, p. 83 (1884); 2, p. 361 (1897). - Hamps., Cat. Lep. Phal. 1, p. 495 , t. 16, f. 15 (1898).

rabuseulum Dogn., Le Nat. 27, p. 10 (1905).

relegatum Schaus, Ann. \& Mag. Nat. Hist. (8) 7, p. 182 (1911).

reniferum Hamps., Cat. Lep. Phal. 1, p. 505, t. 17, f. 26 (1898).

resina Druce, Ann. \& Mag. Nat. Hist. (7) 5, p. 508 (1900).

Rogersi Druce, Biol. Centr.-Am., Het. 1 , p. 88, t. 9, f. 15 (1884). - Hamps., Cat. Lep. Phal. 1, p. 507 (1898).

rosa Wall., List Lep. Ins. Br. Mís. 1, p. 271 (1854) (Euchromia). - Druce, Biol. Centr.-Am., Het. 1 p. 86 (1884); 2, p. 363 (1897). - Hamps., Cat. Lep. Phal. 1, p. 491, t. 16, f. 17 (1898).

rosadora Dyar, Proc. U. S. Nat. Mius. 38, p. 231 (1910). v. xanthodora Dyar, Proc. U. S. Nat. Mus. 38, p. $233(1910)$.

rosina Walk., List Lep. Ins. Br. Mus. 1, p. 270 (1854) (Euchromia). - Hamps., Cat. Lep. Phal. 1, p. 492 , t. 16 , f. 18 (1898).

rhodo phila Druce, Biol. Centr.-Am., Het. 1, p. 80 (1884) (pr. p.).

ab. imprimatum (-a) Walk., List Lep. Ins. Br. Mus. 31, p. 305 (1864) (Carales). - Hamps., Cat. Lep. Phal. 1, p. 492 (1898).

rufieolle(-is) Lathy, The Ent. 32, p. 120 (1899).

sadana Druce, Ann. \& Aíag. Nat. Hist. (7) 18, p. 81 (1906).

seyton Cram., Pap. Exot.2, p. 5, t. 99, f. B (1777) (Sphinx). - Drucc, Biol. Centr.-Am., Het. 2, p. 361 (1897). - Hamps., Cat. I.ep. Phal. 1, p. 511 (1898).

setosum(-a) Sepp, Surin. Vlind. 1, p. 25, t. 9 (1848) (Phalaena). - Druce, Biol. Centr.-Am., Het. 1 , p. 85 (1884); 2, p. 362 (1897). - Hamps., Cat. Lep. Phal. 1, p. 507 (1898)

Biologie: Sepp, Surin. Vlind. 1, p. 25, t. 9 (1848). - Hamps., Cat. Lep. Phal. 1, p. 507 (1898).

silvius (sylvius) Stoll, Suppl. Cram. p. 65, t. 14, f. 4, 5 (1790) (Sphinx). - Druce, Biol. Centr.-Am., Het. 2, p. 362 (1897). - Hamps., Cat. Lep. Phal. 1, p. 497 (1898).

Ecuador,

Santa

Catharina

(M. C.

Vind.),

Paraguay

Venezuela,

Brit.-

Guayana

Mexico,

Costarica,

Panama

Rio Janeiro

Peru

Costarica

Peru

Colombia

Costarica,

Panama

Mlexico,

Honduras,

Paraná

Mexico

,

Mexico,

Venezuela,

Trinidad,

Rio Janeiro

Venezuela

Peru

Mexico,

Brit.

Hondurss,

Surinam,

São Paulo

Mexico,

Guatemala,

Panama,

Venezuela,

Surinam,

Bahia

Mexico,

Surinam,

Pará,

Amazonas 
lutulentum Möschl., Vcrh. zool.-bot. Ges. Wicn 27, p. 650 , t. 8 , f. 18 (1877).

Biologie: Stoll, Suppl. Cram. p. 65, t. 14, f. 1-3 (1790).

striatum(-n) Druce, Ann. \& Mag. Nat. Hist. (6) 4, p. 86 (1889) (Theages); Biol. Centr.-Am., Het. 2, p. 361 , t. 73 , f. 9 (1897). - Hamps., Cat. Lep. Phal. 1, p. 504 (1898).

sureatum(-a) Dogn., Ann. Soc. Ent. Belg. 41, p. 25 (1897) (Theages).

tarona Hamps., Cat. Lep. Phal. 1, p. 493, t. 17, f. 7 (1898).

tenellulum Schaus, Ann. \& Mag. Nat, Hist. (8) 6, p. 195 (1910).

tessellatum Schaus, Ann. \& MIag. Nat. Hist. (8) 6, p. 194 (1910).

testaceum Druce, Ann. \& Mag. Nat. Hist. (7) 6, p. 65 (1900).

trinita Schaus, Journ. New York Ent. Soc. 9, p. 44 (1901).

tripumetatum Druce, Biol. Centr.-Am., Het. 1, p. 85, t. 9, f. 12 (1884); 2, p. 362 (1897). - Hamps., Cat. Lep. Plial, 1, p. 504 (1898).

Farium(-a) Walk., List Lep. Ins. Br. Mus. 1, p. 266 (1854) (Euchromia). - Butl., Illustr. Het. Br. Mus. 1, p. 49, t. 9, f. 5 (1877). - Drucc, Biol. Centr.-Am., Het. 1, p. 84 (1884). - Hamps., Cat. Lep. Phal. 1, p. 506 (1898).

velutinum Schaus, Journ. New York Ent. Soc. 4, p. 134 (1896). - Hamps., Cat. Lep. Phal. 1, p. 504, t. 17, f. $14(1898)$.

renosum(-a) Schaus, Ann. \& Mag. Nat. Hist. (8) 6, p. 195 (19I0).

vestale(-is) Schaus, Proc. Zool. Soc. Lond, p. 278 (1892) (Theages); Am. Lep. p. 11, t. 1, f. 23 (1892).

xanthoperas Hamps., Cat. Lep. Phal. 1, p. 510, t. 17, f. 13 (1898).

abdominale Drucc, Biol. Centr.-Am., Hct. 1, p. 84 (1884); 2, p. 362 (1897) (nec Walk.).

xanthurum(-a) Schaus, Ann. \& Mag. Nat. Hist. (8) 6, p. $192(1910)$.

zephyrum Schaus, Ann. \& Mag. Nat. Hist. (8) 6, p. 194 (1910).

zizana Dogn., Ann. Soc. Ent. Belg. 4l, p. 24 (1897). Ecuador 121

\section{Metacrocea Dyar.}

Dyar, Proc. U. S. Nat. Mus. 29, p. 173 (1905).

postflava Schaus, Proc. U. S. Nat. Mus. 29, p. 193 (1905). Rio Janeiro 


\section{Apocerea Dyar.}

Dyar, Proc. U. S. Nat. Mus. 29, p. 174 (1905).

sobria Schaus, Proc. U. S. Nat. Mus. 29, p. 193 (1905). Franz.-

Guayana

1

\section{Correbia Herr.-Schäff.}

Herr.-Schäff., Aussereur. Schmett.p. 22 (1853). - Hamps., Cat. Lep. Phal. 1, p. 514 (1898).

Pionia Walk., List Lep. Ins. Br. Mus. 1, p. 255 (1854).

M imica Oberth., Et. Ent. 6, p. 33 (1881).

Biologie: Haase, Bibl. Zool. 8 (2), p. 74 (1893) (Mimicry).

affinis Durce, Biol. Centr.-Am., Het. 1, p. 77, t. 9, f. 4 (1884); 2, p. 356 (1897) (Pionia). - Hamps., Cat. Lep. Phal. 1, p. 517 (1898).

agnonides Druce, Biol. Centr.-Am., Het. 1, p. 77 (1884) (Pionia). - Hamps., Cat. Lep. Phal. 1, p. 516, t. 17, f. 19 (1898).

? elongata(-us) Dogn., Le Nat. (2) 4, p. 91 (1890) (Chloropsinus); Lep. Loja p. 48 , t. 4, f. 3 (189I). Hamps., Cat, Lep. Phal. 1, p. 517 (1898) (huius generis?).

flavata Druce, Ann. \& Mag. Nat. Hist. (8) 3, p. 458 (1909).

lycoides Walk., List Lep. Ins. Br. Mus. 1, p. 256 (1584) (Euchromia). - Butl., Illustr. Het. Br. Mus. 1, p. 47 , t. 8 , f. 10 (1877). - Druce, Biol. Centr.-Am., Het. 1, p. 76 (1884); 2, p. 356 (1897). - Hamps., Cat. Lep. Phal. 1, p. 515, f. 273 (1898).

subochrea Herr.-Schäff., Corresp.-BI. zool.-min. Ver. Regensb. 29, p. 115 (1866).

ab. ceramboides Herr.-Schäff., Aussereur. Schmett. p. 74, f. 265 (1855) (Pionia). - Hamps., Cat. Lep. Phal. 1, p. 515 (1898).

Biologie: Haase, Bibl. Zool. 8 (2), p. 74, t. 13 , f. 101 (1893) (Mfimicry). - Schrottky, Iris 22, p. 128,129 (1909).

minima Druce, Ann. \& Mag. Nat. Hist. (7) 15, p. 465 $(1905)$.

Oberthueri Hamps., Cat. Lep. Phal. 1, p. 515 (1898).

lycoides Oberth., Et. Ent. 6, p. 33, t. 10, f. 9 (1881) (Himica) (nec Walk.).

Biologie: Haase Bibl. Zool. 8 (2), p. 74 (1893) (Mimicry).

obscura Schaus, Proc. U. S. Nat. Mus. 29, p. 193 (1905).

obtusa Druce, Biol. Centr.-Am., Het. 1, p. 77 (1884) (Pionia). - Hamps., Cat. Lep. Phal. 1, p. 516, t. 17, f. 9 (1898).

raca Druce, Ann. \& Mag. Nat. Hist. (6) 18, p. 37 (1896) (Pionia); Biol. Centr.-Am., Het. 2, p. 356, t. 72, f. 18 (1897). - Hamps., Cat. Lep. Phal. 1, p. 517 (1898).

semitransversa Schaus, Ann. \& Mag. Nat. Hist. (8) 7 , p. $182(1911)$.

Mexico, Guatemala

Nicaragua

Ecuador

Colombia

Cuba, Jamaica,

Mexico,

Brit.-

Honduras,

Panama,

Venezuela,

Ecuador,

RioJaneiro,

Santa

Catharina

(M.C.Vind.),

Paraguay

Venezuela

Peru,

Bolivia

Franz.-

Guayana

Guatemala

Panama

Costarica 
nndulata Druce, Biol. Centr.-Am., Het. 1, p. 77, t. 9, f. 3 (1884); 2, p. 356 (1897) (Pionia). - Hamps., Cat. Lep. Phal. 1, p. 516 (1898).

Biologie: Haase, Bibl. Zool. 8 (2) p. 74 (1893) 12 (Mimiory).

\section{Correbidia Hamps.}

Hamps, Cat. Lep. Phal. 1, p. 517 (1898).

apicalis Schaus, Trans. Am. Ent. Soc. 30, p. 136 (1904). calopteridia Butl., Proc. Zool. Soc. Jond. p. 381 (1878) (Pionia). - Hamps., Cat. Lep. Phal. 1, p. 518, t. 17, f. 22 (1898).

costinotata Schaus, Ann. \& Mag. Nat. Hist. (7) 7, p. 183 (1911).

elegans Druce, Biol. Centr.-Am., Het. 1, p. 78, t. 9, f. 6 (1884); 2, p. 356 (1897) (Pionia). - Hamps., Cat. Lep. Phal. 1, p. 519 (1898).

notata Butl., Trans. Ent. Soc. Lond. p. 45 (1878) (Pionia). - Hamps., Cat. Lep. Phal. 1, p. 518, t. 17, f. 3 (1898).

striata Druce, Biol. Centr.-Am., Het. 1, p. 78, t. 9, f. 5 (1884) (Pionia). - Hamps., Cat. Lep. Phal. 1, p. 519 (1898).

terminalis Walk., List Lep. Ins. Br. Mus. 7, p. 1633 (1856) (Pionia). - Hamps., Cat. Lep. Phal. 1, p. 519, f. 274 (1898).

cimicoides Herr.-Schäff., Corresp.-Bl. zool.-min. Ver. Regensl. 20, p. 116 (1866) (Charidea). calopteridia Druce, Biol. Centr.-Am., Het. 1, p. 77 (1884); 2, p. 356 (1897) (Pionia) (nec Butl.). Biologie: Dew., Nova Acta Acad. Leop.-Carol. 44, p. 252 , t. 1 , f. $14,14 a(1882)$.

tristitia Kaye, The Ent. 44, p. 145 (1911).

8

\section{Scepsis Walk.}

Walk., List Lep. Ins. Br. Mus. 2, p. 285 (1854). - Pack., Proc. Essex Inst. 4, p. 40 (1864). - Hamps., Cat. Lep. Phal. 1, p. 520 (1898).

discopuncta Hamps., Ann. \& Mag. Nat. Hist. (7) 8, p. 175 (1901).

fulvicollis Hübn., Samml. Exot. Schmett. 1, t. 164 (1827) (Glaucopis). - Stretch, Zyg. \& Bomb. N. Am. p. 21 , t. 1, f. 9 (1872). - Noum. \& Dyar, Journ. New York Ent. Soc, 1, p. $10+$ (1893). - Hamps., Cat. Lep. Phal. 1, p. 520 , f. 275 (1898).-Beutenm., Bull. Am. Jlus. Nat. Hist. 10, p. 353, t. 15, f. 13 (1898). - Holl., Moth Book p. 101, t. 13, f. 12 (1903).

semidiaphana Harr., Am. Journ. Sc. 36, p. 318 (1839) (Glaucopis).

v. pallens Hy. Edw., Ent. Am. 2, p. 8 (1886). - Neum. \& Dyar, Journ. New York Ent. Soc., p. 104 (1893). - Hamps., Cat. Lep. Phal. 1, p. 520 (1898).
Mexico, Guatemala

Cuba

Pará

Costarica

Mexico, Costarica,

Panama

Amazonas

Nicaragua,

Panama

Cuba, Mexico, Guatemala, Costarica,

Panama,

Venezuela

Brit. -

Guayana
Bolivia

Vereinigte

Staaten,

Alberta 
Biologie: Coquillett, Gan. Ent. 12, p. 44 (1880). Trans. Dept. Agric. III. 18, Append. p. 171 (1880). - Beutenm., Bull. Am. Mus. Nat. Hist. 10, p. 353 (1898). - Dyar, Proc. U. S. Nat. Mus. 23, p. $264(1900)$.

Packardi Grote, Proc. Ent. Soc. Philad. 4, p. 318 (1865). - Neum. \& Dyar, Journ. New York Ent. Soc. 1, p. 104 (1893). - Hamps., Cat. Lep. Phal. 1, p. 520 (1898).

Mathewi Hy. Edw., Proc. Calif. Acad. 5, p. 184 (1874).

v. Cocklei Dyar, Proc. U. S. Nat. Mus. 27, p. 792 (1904).

subhyalina Hamps., Ann. \& Mag. Nat. Hist. (7) 8, p. 175 (1901).

Wrighti(-ii) Streteh, Ent. Am. 1, p. 101 (1885). Neum. \& Dyar, Journ. New York Ent. Soc. 1, p. 104 (1893). - Hamps., Cat. Lep. Phal. 1, p. 521, t. 17, f. 6 (1898). - Holl., Moth Book p. 101 , t. 13 , f. 13 (1903).

ab. gravis Hy. Edw., Ent. Am. 2, p. 8 (1886). Neum. \& Dyar, Journ. New York Ent. Soc. 1, p. 104 (1893). - Hamps., Cat. Lep. Phal. 1, p. 521 (1898).

Biologie: Coquillett, Journ. Netw York Ent. Soc.6, 5 p. 249 (1898).

\section{Propyria Hamps.}

Hamps., Cat. Lep. Phal. 1, p. 521 (1898).

atroxantha Schaus, Proc. U. S. Nat. Mus. 29, p. 193 (1905). flora Schaus, Ann. \& Mag. Nat. Hist. (8) 7, p. 183 (1911). Normani Schaus, Ann. \& Mag. Nat. Hist. (8) 7, p. 613 (1911).

ptychoglene Hamps., Gat. Lep. Phal. 1, p. 521, f. 276 (1898).

a equalis Druce, Biol. Centr.-Am., Het. 1, p. 129 (1884) (Ptychaglene) (nec Walk.).

Schausi Dyar, Journ. New York Ent. Soc. 6, p. 34 (1898) (Lycomorpha). - Hamps., Cat. Lep. Phal. 1, p. 521 (1898).

fulgens. Neum. \& Dyar, Journ. New York Ent. Soc. 1, p. 103 (1893) (Lycomorpha) (nec Hy.

5 Edw.)

Nordwestl.

Vereinigte

Staaten

Brit.-

Columbia

Bolivia

Kaliformien
Mexico

Costarica

,

Mexico, Guatemala

\section{Lycomorpha Harr.}

Harr., Am. Journ. Sc. 36, p. 317 (1839). - Pack., Proc. Essex Inst. 4, p. 43 , t. 2, f. 4 (1864). - Hamps., Cat. Lep. Phal. 1, p. 522 (1898). Anatolmis Pack., Proc. Essex Inst. 4, p. 45 (1864). Biologie: Haase, Bibl. Zool. (8) 2, p. 73 (1893) (Mimiory).

Drueel Hamps., Ann. \& Mag. Nat. Hist. (7) 8, p. 175 Mexico (1901).

fulgens Hy., Edw. Papilio 1, p. 116 (1881) (Anatolmis). Neumexico, - Neum. \& Dyar, Journ. New York Ent. Soc. 1, Arizona 
p. 103 (1893). - Hamps., Cat. Lep. Phal. 1, p. 522 , t. 17 , f. 11 (1898).

Grotei Pack., Proc. Esscx Inst. 4, p. 47 (1864) (Anatolmis). - Stretch, ZJg. \& Bomb. N. Am. p. 44, t. 2, f. 4 (1872). - Neum. \& Dyar, Journ. New York Ent. Soc. 1, p. 103 (1893). - Hamps., Cat. Lep. Phal. 1, p. 522 (1898). - Holl., Mloth Book p. 101, t. 13, f. 14 (1903).

Palmerii Pack., Rep. Peabody Acad. 4, p. 84 (1872).

ab. pulchra Dyar, Journ. New York Ent. Soc. 6, p. 34 (1898). - Hamps., Cat. Lep. Phal. 1, p. 522 (1898).

pholus Drury, Ill. Exot. Ent. 2, p. 49, t. 28, f. 3 (1773). (Sphinx). - Stretch, Zyg. \& Bomb. N. Am. p. 42 , t. 2 , f. 3 (1872). - Neum. \& Dyar, Journ. New York Ent. Soc. 1, p. 102 (1893). - Hamps., Cat. Lop. Phal. 1, p. 523, f. 277 (1898). Beutenm., Bull. Am. Mus. Nat. Hist. 10, p. 355, t. 15, f. 9 (1898). - Holl., Motl Book p. 101, t. 13, f. 15 (1903).

จ. miniata Pack., Rep. Peabody Acad. 4, p. 84 (1872). - Neum. \& Dyar, Journ. New York Ent. Soc. 1, p. 102 (1893). - Hamps., Cat. Lep. Phal. 1, p. 523 (1898).

Biologie: Harr., Am. Journ. Sc. 36, p. 318 (1839); Ins. Inj. Veget., Flints ed. p. 341 (1862). Haase, Bibl. Zool. 8 (2), p. 73, t. 13, f. 100 (1893) (Mimicry). - Dyar, Proc. Boston Soc. Nat. Hist. 27, p. 137 (1896); Psyche 8, p. 82 (1897). - Hamps., Cat. Lep. Plal. 1, p. 523 (1898). - Beutenm., Bull. Am. Mus. Nat. Hist. 10, 4 p. 355 (1898).

\author{
Westl. \\ Vereinigte \\ Staaten \\ Canada, \\ Vereinigte \\ Staaten
}

Kalifornien

\section{Ctenucha Kirby.}

Kirby, Fauna Bor.-Am. 3, p. 305 (1837). - Pack., Proc. Essex Inst. 4, p. 33, t. 1 (1864). - Hamps., Cat. Lep. Phal. 1, p. 523 (1898).

Compsoprium Blanch., in Gay, Nat. Hist. Chil., Zool. 7, p. 67 (1852).

Caralisa Walk., List Lep. Ins. Br. Mus. 7, p. 1660 (1856).

Euctenucha Grote, Bull. Buff. Soc. 1, p. 33 (1873).

afflnis Druce, Biol. Centr.-Am., Het. 1, p. 83 (1884); 2 , p. 359 , t. 73 , f. 3 (1897). - Hamps., Cat. Lep. Phal. 1, p. 530 (1898).

albolineata Druce, Ann. \& Mag. Nat. Hist. (7) 13, p. 241 Peru (1904)

albipars Hamps., Ann. \& Mag. Nat. Hist. (7) 8, p. 175 (1901).

annulata Schaus, Trans. Am. Ent. Soc. 30, p. 137 (1904).

aymara Schaus, Proc. Zool. Soc. Lond. p. 285 (1892) Peru (Gangamela); Am. Lep. p. 16, t. 2, f. 9 (1892). - Hamps., Cat. Lep. Phal. 1, p. 525 (1898).

bilormis Dogn., Ann. Soc. Ent. Belg. 51, p. 224 (1907). Peru 
braganza Schaus, Proc. Zool. Soc. Lond. p. 288 (1892) (Melanchroia); Am. Lep. p. 8, t. 2, f. 26 (1892). - Hamps., Cat. Lep. Phal. 1, p. 526 (1898).

brunnea Stretch, Zyg. \& Bomb. N. Am. p. 30, t. 1, f. 11 (1872). - Neum. \& Dyar, Journ. New York Ent. Soc. 1, p. 106 (1893). - Hamps., Cat. Lep. Phal. 1, p. 529 (1898). - Holl., Moth Book p. 102 , t. 11, f. 6 (1903).

circe Cram., Pap. Exot. 3, p. 124, 147, t. 263, f. D, t. 274, f. E (1780) (Bombyx). - Druce, Biol. Centr.Am., Het. 1, p. 83 (1884). - Hamps., Cat. Lep. Phal. 1, p. 526 (1898).

affin is Boisd., Lép. Guitem.p. 88 (1870) (Euagra). pylotis Möschl., Verh. zool.-bot, Ges. Wien 27, p. 646 (1877) (nec Fabr.).

clavia Druce, Proc. Zool. Soc. Lond. p. 383 (1883) (Hop. larctia). - Hamps., Cat. Lep. Phal. 1, p. 531, t. 17, f. 27 (1898).

eressonana Grote, Proc. Ent. Soc. Philad. 2, p. 64, t. 8, f. 5 (1863). - Stretch, Zyg. \& Bomb. N. Am. p. 28, t. 1, f. 14 (1872). - Neum. \& Dyar, Journ. New York Ent. Soc. 1, p. 106 (1893). - Dyar, Can. Ent. 31, p. 159 (1899). - Grote, ib. 34, p. 104 (1902); 35, p. 7 (1903). - Holl., Moth Book p. 102 , t. 13 , f. 21 (1903).

ab. lutea Grote, Gan. Fnt. 34, p. 104 (1902).

₹. sanguinaria Strecker, Rep. Chief of Engineers, Append. p. 1858, t. 2, f. 2 (1878). - Neum. \& Dyar, Journ. New York Ent. Soc. 1, p. 106 (1893). - Druce, Biol. Centr.-Am., Het. 2, p. 360 (1897). - Harmps., Cat. Lep. Phal. 1, p. 528 (1898).

cyaniris Hamps., Cat. Lep. Phal. 1, p. 525, t. 17, f. 5, (1898).

divisa (devisum) Walk., List Lep. Ins. Br. Mns. 7, p. 1644 (1856) (Compsoprium). - Hamps., Cat. Lep. Phal. 1, p. 527, t. 17, f. 25 (1898).

marita Schaus, Journ. New York Ent. Soc. 4, p. 133 (1896) (Philoros).

editha Walk., List Lep. Ins. Br. Mus. 7, p. 1661 (1856) (Caralisa). - Hamps., Cat. Lep. Phal. 1, p. 527, t. 17 , f. $10(1898)$.

mennisata Dogn., Ann. Soc. Ent. Belg. 44, p. 213 (1900). mortin Schaus, Journ. New York Ent. Soc. 9, p. 44 (1901).

multifaria Walk., List Lep. Ins. Br. Mus. 2, p. 479 (1854) (A pistosia). - Streteh, Zyg. \& Bomb. N. Am. p. 28 , t. 1, f. 12 (1872). - Neum. \& Dyar, Journ. New York Ent. Soc. 1, p. 106 (1893). - Hamps., Cat. Lep. Phal. 1, p. 529 (1898). - Holl., Moth. Book p. 102, t. 13, f. 19 (1903).
Amazonas

(M.C.Vind.),

São Paulo

(M.O.Vind.),

RioJanciro,

Santa

Catharina

Kalifornien

Nicaragua,

Ecuador,

Venezuela,

Surinam,

São Paulo

(M.C.Vind.)

Ecuador

Rooky

Mountains

Rocky
Mountains,
Mexico

Ecuador

Amazonas (M.

C. Vind.),

São Paulo,

Rio Grande

do Sul (M.

C. Vind.),

Argentinien

Haiti

Bolivia

Paraná,

Paraguay

(M.C.Vind.)

Kalifornien 
ab. luteoscapa (-us) Ncum. \& Dyar, Journ. New York Ent. Soc. 1, p. 106 (1893).

Biologie: Hy. Edw., Proc. Calif. Acad. p. 344 (1874).

nautana (nantana) Walk., List Lep. Ins. Br. Mus. 31, p. 282 (1864) (Ammalo). - Butl., Illustr. Het. Br. Mus. 1, p. 54, t. 19, f. 2 (1877). - Hamps., Ann. \& Mag. Nat. Hist. (7) 15, p. 431 (1905). rubicunda Dogn., Le Nat. 27, p. 10 (1905).

palmeira Schaus, Proc. Zool. Soc. Lond. p. 288 (1892) (Melanchroia); Am. Lep. p. 8, t. 2, f. 24 (1892). - Hamps., Cat. Lep. Phal. 1, p. 526 (1898).

popayana Dogn., Hét, nouv. Am. Sud 2, p. 12 (1911). projecta Dogn., Le Nat. 26, p. 67 (1904).

refuIgens Dogn., Ann. Soc. Ent. Belg. 43, p. 327 (1899). Reimoseri Zerny. Iris 26 (1912) (ined.).

rubroseapa(-us) Mén., Cat. Lép. Pétersb. 2, p. 142, t. 14, f. 7 (1857) (Glaucopis). - Neum. \& Dyar, Journ. New York Ent. Soc. 1, p. 106 (1893). - Hamps., Cat. Lep. Phal. 1, p. 529 (1898). - Holl., Moth Book p. 102, t. 13, f. 22 (1903). Walsinghami Hy. Edw., Proc. Calif. Acad. 5, p. 112 (1873). - Stretch, Zyg. \& Bomb. N. Am. p. 213, t. 9, f. I (1572).

ab. ochroscapa (-us) Grote \& Rob., Trans. Am. Ent. Soc. 1, p. 330 (1868). - Stretch, Zyg. \& Bomb. N. Am. p. 29, t. 1, f. 13 (1872). Neum. \& Dyar, Journ. New York Ent. Soc. 1, p. 106 (1893). - Hamps., Cat. Lep. Phal. 1, p. 529 (1898).

corvina Boisd., Anu. Soc. Lint. Belg. 12, p. 71 (1869).

rufieeps Walk, List Lep. Ins. Br. Mus. 2, p. 284 (1854). - Druce, Biol. Centr.-Am., Het. 1, p. 82 , t. 9, f. 8 (1884). - Hamps., Cat. Lep. Phal. 1, p. 528 (1898).

semistria Walk, List Lep. Ins. Br. Mus. 2, p. 321 (1856) (Dioptis). - Hamps., Cat. Lep. Phal. 1, p. 527, t. 17, f. 4 (1898).

tigrina Strecker, Lep. Indig. \& Exot., Suppl. 2, p. 4 (1899). togata Druce, Biol. Centr.-Am., Het. 1, p. 73 , t. 6, f. 8 (1884) (Acreagris). - Hamps., Cat. Lep. Phal. 1, p. 525 (1898).

augusta Hy. Edw., Ent. Am. 3, p. 91 (1887) (Lycomorpha). - Druce, Biol. Centr.-Am., Het. 2 , p. 332 , t. 70 , f. 17 (1896).

venosa Walk., List Lep. Ins. Br. Mus. 2, p. 284 (1854). - Stretch, Zyg. \& Bomb. N. Am. p. 31, t. 1, f. 10 (1872). - Druce, Biol. Centr.-Am., Het. 1, p. 82 (1S84). - Neum. \& Dyar, Journ. New York Ent. Soc. 1, p. 105 (1893). - Hamps., Cat. Lep. Phal. 1, p. 528 (1898). - Grote, Can. Ent. 35, p. 77 (1903). - Holl., Moth Book p. 101, t. 13 , f. 20 (1903).

Реги

Rio Janciro, Rio Grande do Sul, Paraguay

Colombia

Peru

Ecuador

Paraguay

Westl. Vereinigte

Staaten

Mexico

Brasilien (ubi?)

Costarica

Mexico, Guatemala Costarica, Panama

Colorado,

Texas,

Arizona,

Mexico,

Guatemala,

Honduras,

Costarica,

Panama,

Colombia,

(M.C.Vind.),

Venezuela,

Ecuador 
virginica Charp. in Esp. Ausländ. Schmett. 2, Suppl. t. 2, f. 3, 4 (1830) (Sphinx). - Stretch, Zyg. \& Bomb., N. Am. p. 25 , t. 1, f. 15 (1872). - Neum. \& Dyar, Journ. New York Ent. Soc. 1, p. 105 (1893). - Hamps., Cat. Lep. Phal. 1, p. 530, f. 278 (1898). - Beutenm., Bull. Am. Míus. Nat. Hist. 10 , p. 354 , t. 15, f. 5 (1898). - Holl., Moth Book p. 102, t. 13, f. 18 (1903).

latreilliana Kirby, Fauna Bor.-Am. 3, p. 305 (1837).

Biologie: Pack., $7^{\text {th }}$ Rep. Maine Board Agric. p. 168 (1862); Proc. Essex Inst. 4 (1), p. 33, t. 1 (1864). - Lintn., 26th Rep. N. York State Cab. Nat. Hist. p. 155 (1872). - Coquillett, Trans. Dept. Agric. Ill. 18, Append. p. 170 (1880). - Pack., Ann. Netw York Acad. 8, p. 83 (1895). - Dyar, Proc. Boston Nat. Hist. Soc. 27, p. 138 (1896). - Hamps., Cat. Lep. Phal. 1, p. 530 (1898). - Beutenm., Bull. Am. Mus. Nat. Hist. 10, p. 354 (1898).

vittigera(-um) Blanch. in Gay, Nat. Hist. Chil., Zool. 7, p. 67 (1852) (Comproprium). - Burm., Descr. Phys. Rep. Argent. 5, p. 384 (1878). - Berg, An. Soc. Cient. Argent. 13, p. 18 (1882). Hamps., Cat. Lep. Phal. 1, p. 527 (1898).

ab. nivosa Walk., Charact. Undescr. Het. p. 6, (1869). - Hamps., Cat. Lep. Phal. 1, p. 527 (1898).

Biologie: Berg, An. Soc. Cient. Argent. 21, 31 p. 278 (1886).

Vereinigte

Staaten,

Canada

Argentinien,

Chile,

Rio Janeiro

(M. C. Vind.)

\section{Dahana Grote.}

Grote, Can. Ent. 7, p. 175 (1875). - Hamps., Cat. Lep. Phal. 1, p. 531 (1878).

atripeunis Grote, Can. Ent. 7,p. 175 (1875). - Ncum. \& Dyar, Journ. Nct York Ent. Soc. 1, p. 105 (1893). - Hamps., Cat. Lep. Phal. 1, p. 531, f. 279 (1898). - Holl., Moth Book p. 103, t. 13, f. $23(1903)$.

cubana Schaus, Trans. Am. Ent. Soc. 30, p. 136 (1904). Florida 2

\section{Epectoptera Hamps.}

(Epectaptera) Hamps., Cat. Lep. Phal. 1, p. 531 (1898).

discalis Schaus, Proc. U. S. Nat. Mus. 29, p. 194 (1905) discosticta Hamps., Cat. Lep. Phal. 1, p. 532, t. 17, f. 24 (1898).

innotata Dogn., Ann. Soc. Ent. Belg. 43, p. 327 (1899). laudabilis Druce, Ann. \& Mag. Nat. Hist. (6) 18, p. 35 (1896) (Aclytia). - Hamps., Cat. Lep. Phal. 1, p. 532, f. 280 (1898)

umbrescens Schaus, Proc. U. S. Nat. Mus. 29, p. 194 Регu

Peru

Venezuela

Ecuador

Bolivia 


\section{Philorus Walk.}

(Philoros) Walk., List Lep. Ins. Br. Mus. 2, p. 283 (1854). - Hamps., Cat. Lep. Phal. 1, p. 532 (1898).

laura Hamps., Cat. Lep. Phal. 1, p. 533, t. 17, f. 23 (1898). neglectus (-a) Boisd., Voy. Astrolabe, Lép. p. 195, t. 3, f. 8 (1832) (Tipulodes). - Burm., Descr. Phys. Rep. Argent. 5, p. 383 (1878). - Hamps., Cat. Lep. Phal. 1, p. 533 (1898).

nora Druce, Ann. \& Mag. Nat. Hist. (7) 18, p. 81 (1906). obseuratus (-a) Hamps., Ann. \& Mag. Nat. Hist. (7) 8, p. $176(1901)$.

perirroratus (- $\Omega$ ) Hamps., Ann. \& Mag. Nat. Hist. (7) 8, p. $176(1901)$.

rabriceps Walk., List Lep. Ins. Br. Mus. 2, p. 283 (1854) (Ctenucha). - Druce, Biol. Centr.-Am., Het. 1, p. 82 (1884); 2, p. 359 (1897). - Hamps., Cat. Lep. Phal. 1, p. 533, f. 281 (1898).

scepsiformis Hy., Edw. Ent. Am. 3, p. 91 (1887) (Ctenucha). - Druce, Biol. Centr.-Am., Het. 2, p. 359 (1897).

ab. opacus (-a) Boisd., Lép. Guatem. p. 84 (1870) (Ctenucha). - Berg, An. Soc. Cient. Argent. 13, p. 18 (1882). - Hamps., Cat. Lep. Phal. 1, p. 533 (1898). - Schrottky, Iris 24, p. 153 (1910).

6

Bolivia

Mexico (M.

C. Vind.),

Brasilien

(ubi? M.

c. Vind.),

Peru,

Argentinien

Peru

Colombia

Bolivia

Mexico,

Guatemala,

Costarica,

Panama

(M.C.Vind.),

Colombia,

Venezuela,

Amazonas

(M.C.Vind.),

São Paulo,

Rio Janeiro,

Rio Grande

do Sul (M.

C. Vind.),

Paraguay,

Uruguay,

Argentinien

\section{Hyalencerea Bntl.}

Butl., Ann. \& Mag. Nat. Hist. (4) 15, p. 399 (1875). - Hamps., Cat. Lep. Phal. 1, p. 533 (1898).

agylloides Dyar, Proc. U. S. Nat. Mus. 42, p. 50 (1912). Chapmani Klages, Proc. U. S. Nat. Mus. 29, p. 552 (1906).

erythrotela(-us) Walk., List Lep. Ins. Br. MIus. 1, p. 147 (1854) (Glaucopis). - Butl., Mlustr. Het. Br. Mus. 1 , p. 51, t. 7, f. 13 (1877). - Druce, Biol. Centr.-Am., Het. 2, p. 365 (1897). - Hamps., Cat. Lep. Phal. 1, p. 535, f. 283 (1898).

fusiformis Walk., List Lep. Ins. Br. Mus. 7, p. 1629 (1856) (Pampa). - Hamps., Cat. Lep. Phal. 1, p. 535, f. 284 (1898).

trifasciata Butl., Illustr. Het. Br. Mus, 1, p. 49, t. 16, f. 11 (1877) (Scepsis).

gigantea Druce, Biol. Centr.- Am., Het. 1, p. 80, t. 9, f. 7 (1884); 2, p. 358 (1897). (Epanycles). Hamps., Cat. Lep. Phal. 1, p. 535, f. 285 (1898).

Mexico

Venezuela

Honduras,

Venezuela,

Pará

Amazonas

Mexico,

Guatemala,

Panama,

Brasilien

(ubi?, (M.

C. Vind.) 
leucoprocta Dogn., Ann. Soc. Ent. Belg. 53, p. 217 (1909). Franz.-

leucosticta Druce, Ann. \& Mag. Nat. Hist. (7) 15, p. 466 (1905).

luctuosa Möschl., Verh. zool.-bot. Ges. Wien 27, p. 642, t. 8, f. 13 (1877) (Heliura). - Hamps., Cat. Lep. Plaal. 1, p. 534 (1898)

lugubris Schaus, Journ. New York Ent. Soc. 9, p. 44 Colombia (1901).

morosa Schaus, Ann. \& Mag. Nat. Hist. (8) 6, p. 195 (1910). Costarica

phaeosoma Hamps., Ann. \& Mag. Nat. Hist. (7) 15, Paraguay p. 431 (1905).

picticeps Hamps., Ann. \& Mrag. Nat. Hist. (7) 11, p. 341 (1903).

sororia Schaus, Ann. \& Mag. Nat. Hist. (8) 6, p. 196 (1910).

vulnerata Butl., Ann. \& Mag. Nat. Hist. (4) 15, p. 399 (1875). - Druce, Biol. Centr.-Am., Het. 2, p. 365, t. 73, f. 16 (1897). - Hamps., Cat. Lep. Phal. 1, p. 584, f. 282 (1898). - Schrottky, Iris 15, p. 136 (1902).

myrrhine Burm., Descr. Phys. Rep. Argent. 5, p. 375 (1878); Atlas t. 17, f. 6 (1879) (Glaucopis). 14

Rio Janeiro

Costarica

Mexico,

Panama,

São Paulo

(M.C.Vind.)

Espiritu

Santo,

Paraguay,

Argentinien 


\section{Index.}

\section{Genera.}

Abrochia Herr.-Schäff., 108

Aclytia Hübn., 130

Acridopsis Butl., 137

Aethria Hülun, 102

Aethriopsis Schrottky, 103

Agaphthorn Meyr., 5

Agerocha Hübn., 76

Agunaix Hamps., 110

Agyrta Hübn., 134

Agyrtidia Hamps., 94

Amata Fabr., 12

Amycles Herr.-Schäff., 115

Amycles Butl., 51

Anace Walk., 41

Anaphlebia Feld., 117

Anatolmis Pack., 147

Andrenimorpha Butl., 57

Androcharta Feld., 113

Antichloris Hübn., 116

Apisa Walk., 41

Apocerca Dyar, 145

Argyroides(-eides) Butl., 103

Aristadaema Wallengr., 67

Asinusca Wallengr., 12

Asinutea Wallengr., 12

Atyphopsis Butl., 117

Autochloris Hübn., 55

Autochloris Hamps., 124

Balacra Walk., 45

Bombiliodes Hamps., 55

Bombopsyche Hamps., 50

Burtia Grote, 105

Buthysia Wallengr., 12

Cacosoma Feld., 4

Cacostatia Hamps., 124

Callagra Butl., 132

Callicarus Grote, 121

Callitomis Butl., 4

Callopepla Hamps., 125
Calonotus(-os) Hübn., 98

Caralisa Walk., 148

Centronia Möschl., 112

Coramidia Butl., 114

Cercophora Herr.-Schäff., 100

Cercopimorpha Butl., 1 I0

Ceryx Wallengr., 5

Charidea Butl., 126

Chloromeles M[cyr., 32

Chloropsinus Butl., 123

Clilorostola Hamps., 123

Chrostosoma Hübn., 64

Chrysocale Walk., 97

Chrysostola Herr.-Schäff., 108

Coenochromia Hübn., 12

Compsoprium Blanch., 148

Copaena Herr.-Schäff., 94

Corematura Butl., 101

Coreura Walk., 129

Correbia Herr.-Schäff., 145

Correbidia Hamps., 146

Corydalla Walk., 134

Cosmosoma Hübn., 67

Ctenandra Feld., 32

Ctenucha Kirby, 148

Cyanopepla Clem., 126

Dahaua Grote, 151

Dasysplinx Feld., 57

Decimia Walk., 42

Delphyre Walk., 135

Desmidocnemis M[ösch]., 107

Desmidocnemis Hamps., 106

Desmotricha Hamps., 129

Diabaena Feld., 118

Diaxanthia Hamps., 125

Didasys Grote, 105

Dinia Walk., 99

Dipteromorpha Feld., 115

Diptilum(-on) Prittw., 106

Dixophlebia Butl., 76 
Drucea Kirby, 121 Dycladia Feld., 86 Dysauxes Hübn., 37

Ecdemus Herr.-Schäff., 110

Echoneura Butl., 80 Empyreuma Hübu., 122

Endera Walk., 84

Enioche Druce, 58

Enope Walk., 92

Epanycles Butl., 111

Epectoptera (Epectapt.) Hamps., 151

Episcepsis Butl., 112

Epitoxis Wallengr., 35

Eressa Walk., 31

Eressades Betli.-Baker, 41

Eriphia Herr.-Schäff., 116

Eriphia Butl., 114

Eriphioides Kirby, 114

Erithales Pocy, 137

Erruca Walk., 67

Euagra Walk., 132

Euccrcum(-on) Hübn., 137

Euchromia Hïbn., 86

Euctenucha Groto, 148

Eumenognster Her'r.-Schäff., 107

Eunomia Hübn., 60

Eupyra Herr.-Schäff., 125

Eurota Walk., 84

Eutomis Hübn., 5

Fregella Walk., 84

Galethalea Butl., 137

Gaugamela (Gangamela) Walk, 131

Glaucopis Burm., 67 .

Glaucopis Hübn., 12

Gundlachia Herr.-Schäff., 105

Gymnelia Walk., 57

Gymnopoda Feld., 80

Haematerion Herr.-Schäff., 99

Hebena Walk., 42

Heliura Butl., 136

Herea Walk., 107

Heterodontia Fold., 64

Hexaneura Wallengr., 42

Hippola Walk., 91

Hira Walk., 86

Histiaea Walk., 92

Holoplioea Hamps., 78

Homoeoccra Fcld., 52

Homoneuronia Dyar, 102

Horama Hübn., 121

Hyalaethea Butl., 34

Hyaleucerea Butl., 152

Hyalomis Hamps., 106

Hyda Walk., 55

Hydrusa Walk., 12
Hyela Walk., 59

Hyela Möschl., 102

Hypatia Kirby, 79

Hyperphara Hamps., 133

Hypocharis Hamps., 80

Hypocladia Hamps., 105

Hysia Walk., 79

Ichoria Butl., 77

Ilipa Walk., 67

Illipula Walk., 116

Isanthrene Hübn., 53

Ixylasia Butl., 123

Laemocharis Herr.-Schäff., 64

Lagaria Walk., 67

Lasioprocta Wallengr., 99

Lasiosceles Hamps., 57

Leucopleura Hamps., 122

Leucotmemis Butl., 65

Loxophlebia Butl., 62

Loxozona Hamps., 120

Lycomorpha Harr., I47

Lycorea Walk., 59

Lymire Walk., 120

Macrocneme Hübn., 94

Mallodeta Butl., 59

Mallostethus Butl., 120

Marecidia Schaus, 122

Marissa TValk., 60

Mastigocera Harr., 121

Meganaclia Auriv., 40

Megapisa Auriv., 45

Mclisa Walk., 47

Mesolasia Hamps., 99

Mesothen Hamps., 63

Metacrocen Dyar, 144

Metaloba Hamps., 84

Metarctia TValk., 42

Metastatia Butl., 108

Methysia Butl., 116

Metriophyla Butl., 118

Mevania Walk., 133

Micragra Hamps., 133

Micragyrta Butl., 83

Microgiton Feld., 133

Micronaclia Hamps., 36

Mimagyrta Hamps., 62

Mimica Oberth., 145

Mochloptera Butl., 65

Mydromera Butl., 132

Mydropaslea Hamps., 96

Nyopsyche Hamps., 9

Myrmecopsis Newm, 48

Mystrocneme Herr.- Schäff., 76

Naclia Boisd., 37

Napata Walk., 118 
Neacerea Hamps., 135

Neëressa Hamps., 31

Neotrichura Druce, 52

Nepe Walk., 132

Nodoza Schaus, 135

Nyridela Lucas, 65

Olina Walk., 135

Orcynia Walk., 48

Paraceryx Beth-Baker, 9

Paraethria Hamps., 103

Paralacthia Hamps., 31

Paramelisa Auriv., 47

Paramya Hamps., 51

Parastatia Hamps., 133

Passineura Butl., 114

Patreliura Hamps., 120

Percote Walk., 130

Pezoptera (Pezaptera) Butl., 101

Phaenarets Druce, 124

Phneo Neum. 96

Phaeosphecia Hamps., 47

Phario Neum., 96

Phalanna Walk., 86

Phara Walk., 135

Phia (Pheia) Walk., 61

Philorus(-os) Walk., 152

Phoenicoprocts Hamps., 59

Phoeniostacta Hamps., 77

Phylloccia Guér., 121

Pionia Walk., 145

Poecilosoma Hübn., 76

Poliopastea Hamps., 98

Pompiliodes Hamps., 51

Pompilopsis Hamps., 50

Procalypta Butl., 131

Propyria Hamps., 147

Psendaclytia Butl., 117

Pseudapiconoma Auriv., 45

Pseudargyroides(-eides) Klages, 110

Pseudmelisa Hamps, 47

Pseudoceryx Rothsch., 34

Pscudocharidea Druce, 129

Pseudocharis Druce, 77

Pseudomelisa Hamps., 47

Pseudomya Hübn., 77

Pseudonaclia Bnti., 36

Preudopompilia Hamps., 123

Pseudosphenoptera Butl., 123

Pseudosphex Hübn., 48

Psichotoe Boisd., 4

Psilopleurn Hamps., 79

Psoloptera Butl., 84
Psychothoc Boisd., 4

Pterygopterus Butl., 111

Ptychotrichus(-os) Schaus, 135

Rizia Kirby, 67

Rhynchopyga Feld., 79

Sansaptera Schaus, 58

Sarosa Walk., 56

Saurita Herr.-Schäff., 80

Sauritinia Dyar, 83

Sccna Walk., 83

Scepsis Walk., 146

Schasiura Butl., 124

Sciopsyche Butl., 117

Scytale Feld., 102

Sesiura Hamps., 108

Sphecopsis Feld., 48

Sphecosoma Butl., 49

Sphenoptera Feld., 92

Stictonaclia Hamps., 35

Streptophlebia Hamps., 10

Symphlebomis Hamps., 10

Syntomeida Harr., 91

Syntomis Ochs., 12

Syntomoides Hamps., 5

Syntrichura Butl., 107

Telioneura Feld., 110

Teucer Kirby, 110

Theages Walk., 137

Thrinacia Butl., 80

Thylacoptera Hamps., 10

Thyretes Boisd., 40

Thyrogoniae Hamps., 40

'Thyrosticta Hamps., 36

Tipulodes Boisd., 125

Trianeura Butl., 32.

Trichaeta Swinh., 10

Trichela Herr.-Schäff., 92

Trichodesma Hamps., 129

Trichromia Hübn., 120

Trichura Hübn., 100

Tritonaclia Hamps., 39

Uraga Walk., 131

Urolasia Hamps., 108

Uxela Walk., 131

Xanthomis Hamps., 92

Xanthopleura Hamps., 123

Zagaris Walk., 42

\section{Species, Varietates.}

abdominalis Walk. (Corematura), 101 | abdominale(-is) Schaus(Sphecos.), 49 abdominale Druce (Eucereum), 144 abdominale(-is) Walk. (Eucer.), 137 
abuorme(-is) Hamps., 49

achemon Fabr., 67

achemonides Dogn., 67

aclytioides Hamps., 135

acquiguttata Dogr., 96

acrospila Feld., 18

acrospila Leceh, 23

acroxantha Perty, 65

actea Swinh. 19

acuminata Walk. (Homoeocera), 52

acuminata Walk. (Ioxozona), 120

acuminata Sncll. (Syntomis), 25

acutior Feld., 56

ada Herr.-Schäff., 67

Adamsi Lathy, 129

adjuncta Walk., 27

admotum(-a) Herr.-Schäff., 67

adonis Druce, 94

adusta Fcld., 115

advena Druce, 67

aeacus Cram., 94

aeagrus Cram., 99

aecyra Herr.-Schäff., 59

aelia Sohaus, 113

aemulina Butl., 88

aenata(-us) Schaus, 51

aeolum Hamps., 137

aequalis Walk. (Abrochia), 109

aequalis Druce (Propyria), 147

aequiguttata Dogn., 96

aestiva Butl., 134

aetolia Druce, 137

affinis Druco (Correbia), 145

affinis Druce (Ctenucha), 149

affinis Klages (Macrocneme), 94

affinis Moore (Eressa), 32

affinis Rothsch. (Argyroides), 103

affinis Rothsch. (Balacra), $\mathbf{4 5}$

affinis Rothsch. (Ceryx), 8

affinis Rothsch. (Eumenogaster), 107

affinis Boisd. (Ctenucha), 148

afflicta Druce, 81

afflicta Walk., 82

africana. Butl., 86

agatha Oberth., 36

agnes Oberth., 35

agnomides Druce, 145

agylloides Dyar, 152

agyrtidia Hamps., 126

alba Druce, 138

albapex Druce (Teucer), 111

albapex Hamps. (Syntomis), 19

Alberti Rothsch. (Hyalacthea), 34

Alberti Rothsch. (Syntomis), 27

albicincta Dogn. (Loxophlebia), 62

albicincta Hamps. (Desmotrich.), 129

albicincta Hamps. (Epitoxis), 35

albicincta Schaus (Phaeo), 96

albicornis Rothsch., 19 albicorpus Kaye, 76

albifasciata Butl., 92

albifrons Moore (Syntomis), 19

albifrons Schaus (Mesothen), 63

albifrons Möschl. (Loxophlebia), 62

albifrontalis Pagenst., 11

albigutta Schaus, 65

albolineata Druce, 148

albimacula Walk., 5

albimaculata Hamps., 118

albomarginata Druce, $5 \mathbf{l}$

albinotum(-a) Dogn., 137

albipars Hamps., 148

albipennis Herr.-Schäff., 120

albiplaga Walk. (Napata), 118

albiplaga Walk. (Trichaeta), II

albipuncta Druce (Hypoclad.), 105

albipuncta Druce (Urolasia), 108

albipuncta Hamps, (Ceryx), 6

albipuncta Hamps. (Thylacopt.), 10

albipunctata Lathy, 114

albisigna Walk., 61

albosignata Walk., 11

albisparsa Hamps., 134

albistriga Schaus, 130

albitarsia Hamps., 94

albiventa(-us) Drucc, 135

albiventris IValk., 66

album(a) Druce, 138

alecton Cram., 116

alesa Druce, 94

aleus Schaus, 70

aliaria Druce, 101

alicia Butl., 12, 16

aliena Walk., 51

Alluaudi Oberth., 9

almon Cram. (Autochloris), 55

almon Hamps. (Ixylasia), 124

alonzo Butl., 126

amadis Schaus, 138

amata Druce, 126

amazonica Butl., 93

amazonula(-oula) Boisd., 35

amboinica Hamps., 88

amoena Möschl., 86

anroenaria Swinh., 27

ampla Walk., 8

amplificata Saalm., 35

analicinctum(-a) Rothsch., 67

analis Schaus (Aethria), 102

analis Sclırottky (Phoenicoprocta), 59

anapera Mab., 36

anastasia Oberth., 35

ancilla L., 38

ancilla Esp., 38

Andersoni Moore, 23

andromacha Fabr., 102

ancpsia Meyr., 27 
aner Hamps., 102

Angasi Druce, 92

angelica Butl., 132

angusta Butl., 81

angustatum(-a) Möschl., 49

angustimargo Schaus, 67

angustipenna Lucas, 33

angustipennis Rothsch., 103

anna Butl., 14

annetta Butl. 19

annexum(-a) Hcrr.-Schäff., 67

annosa Walk., 32

annulata Fabr. (Syntomis), 19, 27

annulata Schaus (Ctenucha), 148

anoxanthium(-a) Druce, 67

Ansorgei Rothsch., 35

antennatus(-a) Rothsch., 98

anthraciformis Wallengr., 5

anthracina Klages (Saurita), 80

anthracina Walk. (Amycles), 115

antiochena Led., 16

antipolo Semp., 10

antitheta Meys., 27

antonia Druce, 138

aoris Möschl., 138

apenninum(-a) Druce, 67

apericns Walk., 32

aperta Walk., 28

aperta Meyr., 29

aperta Turner, 30

a picalis Pothsch. (Teucer), 111

apicalis Schaus (Correbidia), 146

apicalis Strand (Syntomis), 13

apicalis WValk. (Trichaeta), 11

apicalis Herr.-Schäff. (Napata), 118

approximata Walk., 8

appunctatum(-a) Dogn., 138

apricans Walk., 91

arauna Wallengr., 71

archins Stoll, 138

arciizas Druce, 142

arctatom Walk., 49

arenosum Butl., 138

arenosem Druce, 143

arfakensis Rothsch., 28

arganti Druce, 84

argentiflua Martyn. 119

aroa Beth.-Brk. (Ceryx), 8

aroa Beth.-Bak. (Paraceryx), 9

aron Schaus (Autochloris), 55

aroa Schaus (Eucereum), 138

arrogans Walk., 126

artina Butl, 8

aruica Walk., 90

asmodeus Druce, 62

assamensis Zerny, 19

astrifera Butl., 59

astyoche Hübn. (Saurita), 80

astyoche Walk. (Hypatia), 79 atalanta Druce, 118

alava Druce, 135

atavia Hamps. (Mystrocnemc), 76

atavia Hamps. (Coreura), 129

atavistis Hamps., 47

atereum(-us) Schaus (Diptilum), 106 atereus Cram. (Ceryx), 6

atereus Walk. (Syntomis), 19

aterrima Walk. (Isanthrene), 52

aterrima Sepp (Calonotus), 98

Atkinsoni Moore, 26

atricincta Hamps., 118

atricornis Wallengr., 13

atridorsata Hamps., 109

atriguttum(-a) Druce, 138

atripennis Grote, 101

atroxantha Schaus 147

attenuata Hamps. (Saurita), 80

attenuata Hamps. (Syntomis), 28

attenuata Rothsch. (Syntomis), 15

aucta Lecch (Syntomis), 24

auge I. (Cosmosoma), 68

auge Druce (Cosmosoma), 74

auge Neum. \& Dyar (Cosmosoma), 72

auge Walk. (Dinia), 99

augiades Druce, 103

augusta Druce (Abrochia), 109

augusta H. Edw. (Ctenucha), 150

aurantiaca Dogn. (Mesothen), 63

aurantiaca Jones (Loxophlebia), 62

aurantiaca Rothsch. (Apisa), 41

auranticauda Klages, 117

auranticincta Hamps. (Euchr.), 88

auranticincta Klages (Argyr.), 103

aurantiifrons Rothsch., 19

aurantiipes Rothsch. (Diptilum), 106

aurantiipes Rothsch. (Sphecos.), 49

aurantiobasis Rothsch., 8

aurantiocauda Klages, 117

aurata Schaus (Phaeo), 96

auratus(-a) Walk. (Calonotus), 98

aurea Swinh., 19

aureola Swinh., 19

aurifer(-a) Klages (Pseudoshpex), 48

aurifera Butl. (Trichura), 100

aurifera Herr.-Schäff.(Psoloptera), 84

aurimacula Schaus, 126

auripes Walk. 94

aurofasciata Swinh., 28

Austeni Moore, 27

austera Dogn., 91

auxo L. (Agyrta), 134

ausco Walk. (Euagra), 132

aymara Schaus, 148

azetas Druce, 127

azia Druce, 52

azurea Lathy (Metastatia), 108

azurea Walk. (Euagra), 132 
bacchans Schaus, 96

bactriana Ersch., 16

Baeri Rothsch. (Eurota), 84

Baeri Rothsch. (Phoenicoprocta), 59

bajuea Swinh., 16

baleris Dyar, 138

balia.-um) Hamps., 136

barbata Schrottky, 103

Baroni Rothsch. (Cosmosoma), 68

Baroni Rothsch. (Gymnelia), 58

Baroni. Rothsch. (Gymnelia), 58

basalis Walk. (Ceryx), 8

basalis Walk.(Pseudosphenopt.), 123

basifera Waik. (Isanthrene) 53

basifera Walk. (Trichaeta), 11

basifulva Schaus, 84

basigera Walk., 28

basilutea Walk., 55

basimacula Hamps., 126

basirufa Swinh., 24

Batesi(-ii) Butl. (Cosmosoma), 68

Batesi Druce (Balacra), 45

Batesi Fold. (Histiaea), 93

beatrix Drucc, 68

beatum(-ra) Butl., 68

befana Skinner, 91

Belae Grotc. 105

bclla Gućr. (Cyrnopepla), 126

bella Kaye (Loucotmemis), 65

bellatrix Walk., 93

bellatrix Walk., 93

bellula Mab., 87

benitensis Holl., 42

berinda Moore, 19

bertha Druce, 127

bibia Walk, 82

bicinctr Koll., 19

bicolor Dogn. (Saurita), 80

bicolor Rothsch. (Micronaclia), 36

bicolor Walk. (Syntomis), 28

bicolora Walk., 44

bifasciata Auriv. (Pseudonaclia), 36

bifasciata Hopff. (Syntomis), 13

biformis Dogn., 148

bijuncta Walk., 55

biplagata Snell., 11

biplagiata Rothsch., 11

bipuncta Hamps., 80

biradiata Feld., 80

Birchelli Druce, 138

biscriatum Schaus, 68

bisigna Kaye, 61

bivitreata Hamps., 34

bivittata Walk. (Trichaeta), 10

bivittatum(-a) Walk. (Diptilum) 106

bivulnerata Grote, 127

Blanchardi Pouj., 33

blandina Obcrth., 9

Boenninghauseni Rothsch., 56
Boettgeri Druce (Napata), 118

Boettgeri Rothsch. (Gymnelia), 58 bogotense(-is) Feld., 68

Boisduvali Montr., 90

bolivarense(-is) Klages, 67

Bolivari Schaus, 68

boliviana Druce (Argyroides), 103

boliviana Druce (Histiaea), 93

bombiformis Rothsch., 52

borealis Rothsch., 11

borguensis Hamps., 13

bourica Boisd., 89

brachypecten Hamps., 7

braco Herr.-Schäff., 103

braconida Kaye, 79

braconoides Walk., 68

bractea Möschl., 130

braganza Schaus, 149

brasiliense Rothsch. (Cosmosoma), 68

brasiliensis Butl. (Androcharta), 113

brevipennis Butl., 111

Bricenoi Rothsch. (Cosmosoma), 68

Bricenoi Rothsch. (Ichoria), 77

brilliantina Rothsch., 89

Brinkleyi Rothsch., 68

brithyris Druce, 19

Broadwayi Schaus, 118

brodea Schaus, 108

bromus Cr. (Cosmosoma), 68

bromus Walk. (Isanthrene), 54

broteas Schaus, 62

brunnea Druce (Delphyre), 135

brunnea Grünberg (Balacra), 46

brunnea Stretch (Ctenucha), 149

brunneus(-a) Hamps. (Touccr), 111

Buchwaldi Rothsch., 68

Buckleyi Druce (Cyanopepla), 127

Buckleyi Druce (Gymnelia), 58

bura Herr.-Schäff. (Cosmosoma), 69

bura Butl. (Loxophlebia), 62

burica Boisd., 89

burra Schaus. \& Clem., 42

Burtti Dist., 13

Butleri Mab. (Tliyrosticta), 36

Butleri Möschl. (Ceramidia), 114

Butleri Röb. (Euchromia), 88

caca Hübn., 115

caecum Hamps, 68

caelestina Cram., 132

caelipennis Walk., 89

caelipunctata Lathy, 89

caerulea(-la) Dogn. (Eurgra), 132

caerulea Druce (Holophaea), 78

caeruleicorpus Schaus, 63

caeruleifascia Walk., 45

ceeruleonigra Schaus, 96

caerulescens Dogn. (Macrocneme), 94 
cacrulescens Druce (Syntomis), 13 caeruleus Hamps., 111

caffra Wallengr., 40

caffraria Herr.-Schäff., 10

Cajetani Rothsch., 53

calcarata Walk., 48

calita Dogn., 105

calopteridia Butl., 146

calopteridia Druce, 146

Cambonei Oberth., 37

cana Hall., 41

cameseens Walk., 41

Cantori Moore, 19

capistrata Fabr., 55

capys Cram., 120

capysca Schaus, 112

capyscoides Dogn., 112

carabayanum Rothsch. (Cosmos.), 68 carabayanum(-a) Schaus(Eucer-),138 cardinalis Hamps. (Cosmosoma), 68 cardinalis Rothsch. (Saurita), 80 cardinalis(-a) Schaus (Chrostos.), 64 carmania Druce, 111

carminata Hamps., 55

carnea Hamps, 40

carnicauda Butl., 102

camicauda Hainps, 102

carolina Hy. Edw., 139

caryocatacies Wallengr., 6

casea Dogn., 138

caspin Stdgr., 16

cassandra L., 80

cassandra Druce, 81

cassotis Druce, 113

castra Hamps., 118

castrensis Jones, 121

catalcuca 3utl., 115

catastibina ButI., 81

catena Wileman, 6

catherina Schaus, 63

catoria Swinh., 32

caudala Fabr., 100

caudatula Feld., 117

caunus Cram., 55

caurensis Klages (Ceramidia), 115

caurensis Klages (Eumenogast.), 108

caurensis Klages (Macrocneme), 94

caurensis Klages (Pseudargyr.), 110

caurensis Klages (Pseudosphex), 48

caymanensis Hamps., 60

celebensis Butl., 88, 90

celebesa Walk., 28

centrale(-is), Walk., 68

cephalena Drucc, 96

cepheus Cram., 93

ceramboides Herr.-Schäf., 145

ccrbera L. (Syntomis), 13

cerbera Sulz. (Syntomis), 24

cerberus Pall. (Trichura), 100 ceres Druce (Argyroides), 104

ceres Oberth. (Syntomis), 13

cermeua Dogn., 69

cerymica Druce, 132

chalciope Hübn, 65

chalcipleurus(-a) Hamps., 98

chalcodon Druce, 138

chalcomedusa Druce, 77

chalconitis Druce, 65

chalcosticta Butl., 72

chalcoviridis Hamps., 115

chalybea Hamps. (Napata), 118

chalybca(-bsa)Hamps.(Pseudom.),47

chalybea Hamps. (Napata), 118

Chamboni Dogn., 59

Championi Druce, 53

Chapmani Klages, 152

chea Druce, 6

chelidon Druce, 127

chena Druce, 134

cherra Moore, 6

chica Hainps, 132

chimaera Rothsch., 58

chiriquense(-is) Rothsch, 69

chloë Druce, 127

chlorocera Hamps, 19

chloroleuca Walk., 12

chlorometis Meyr., 28

chloroplegia Drucc, 115

chloroscia Hamps., 13

Cholmlei Hamps, 13

choneutospila Tumer, 28

chroma Swinh., 28

chromatica Turuer, 28

chrostosomidis Schaus, 77

chryscis Druce, 98

chrysis Hübn., 76

chrysitis Guér., 94

chrysocraspis Hamps, 106

chrysogastra Perty, 101

chrysonota Hamps., 51

chrysopyga Plötz, 41

chrysorrhoea Hamps., 59

chrysotarsia Hamps., 94

chrysozona Hamps, 13

cimicoides Herr.-Sohäff., 146

cimonis Schaus, 138

cincta Montr. (Fuchromia), 89

cincta Schaus (Autochloris), 55

cincta Schaus (Cosmosoma), 69

cinctata Hamps., 62

cincticollis Feld., 118

cinctipennis Walk., 127

cinctum(-a) Schaus (Cosmosom.), 69

cinctum Schaus (Eucereum), 138

cinctum Hamps. (Eucereum), 141

cinctutum(-a) Schaus, 69

cinerea Butl., 118

cinereocostata Holl., 41 
cingla Schaus, 61 cingulata Weber (Syntomis), 19 cingulata Butl. (Syntomis), 30 cingulata Wallengr. (Syntomis), 22 ciugulatum Butl. (Cosmosoma), 75 cingulosus Feld., 133

cinnamomea Wallengr., 44 cinyras Schaus, 94

circe Cr., 149

citrinum Rothsch., 69

clandestina Zeruy, 81

Claremonti Heyl., 6

claripennis Butl., 113

clavata Walk., 59

clavia Drucc, 149

clavipennis Butl. (Pterygopter.), 111

clavipennis Druce (Pterygopter.), 111

clavipes Boisd., I21

Clementsi Schaus (Eucereum), 138

Clementsi Hamps. (Syntomis), 28

cleta Plötz, 41

elimacina Butl., 65

cloclia Borkh., 17

clusia Drucc, 80

coarcta Drury, 100

coarctata Cram., 100

cocandica Ersch., I6

coccineum Butl., 74

cocho Schaus, 58

Cocklci Dyar, 147

coel-, coelul-, vide : cael-, caerul-

coenobita Möschl., 138

cognatum(-a) Walk., 49

collaris Swinh. (Euchromia), 89

collaris Fabr. (Syntomis), 20

collaris Herr.-Scliäff. (Herea), 107

collocata Walk., 55

Colombiae Butl., 93

colombina Fabr., 60

colona Schaus, 69

Columbiae Butl., 93

columbiana Rothsch., 53

columbina Dyar (Eriphioides), 114

colunibina Fabr. (Ennomia), 60

completa Walk., 56

coinplicatum Butl., 139

compsa Jord., 45

compta Walk. (Syntomis), 20.

compta Sepp (Androcharta), 114

concisa Walk., 81

concurrens Lecch, 21

confine Herr.-Schäff. (Cosmos.), 67

confinc(-js)Herr.-Sclıäff.(Eucer.), 139

continis Walk. (Eressa), 32

confluens Leech (Syntomis), 18

confluens Oberth. (Thyrosticta), 37 congcnera(-gener) Hamps., 13

congoensis Rothsch., 45

conjunctum Möschl., 142 connectens Rothsch., 20

conmexa Walk., 41

colunotata Hamps., 56

Conradti Oberth. (Balacra), 45

Conradti Oberth. (Metarctia), 43

consequa Leech, 20

consimilis Hanmps., 13

consobrina Walk., 109

consociata Walk., 55

consolatum(-a) Walk., 69

cousors Schaus (Eupyra), 125

consor's Walk. (Mallodeta), 59

consortum(-a) Schaus, 138

conspicua Druce (Aclytia), 131

conspicua Schaus (Agyrta), 134

constincta Butl., 5

contermina Walk., 22

contigua Saalm., 37

contractum(-a) Walk., 69

contrasta Beth.-Bak., 42

corallonota Feld., 81

corax Hamps., 97

correbioides Feld., 86

corvicum(-a) Dogn., 69

corvina Boisd. 150

corvus IVallengr., 15

corydon Druce, 123

cosmosomoides Rothsel.. (Abr.), 109

cosinosomoides Rothsch. (Sox.), 62

ccstalis Rothsch., 61

costaricensis Druce, 126

costinotata Sclaus (Correbidia), 146

costinotatum Dogn. (Eucereum), 139

costulatum(-n) Hcrr.-Schäff., 139

costulatum Drucc, 138, 142

crabronoformis Stgr., 53

crabronis Druce, 48

crassa Feld. (Homoeoccra), 52

crassa Feld. (Metarctia), 42

crassum Jenny (Diptilum) 106

crathidinum Rothsch., 69

Crawshayi Hamps, 6

cremnotherma. Lower, 31

crenophylax Holl, 41

creohota Holl., 13

cressonana Grote, 149

creusa I. (Euchromia), 89

creusa Cr. (Syntomis), 24

cribrum Möschl., 143

crocata Herr.-Schäff., 62

craceipes Auriv., 47

croceizona Hamps., 13

cruenta Herr.-Schäff. (Burtia), 105

cruenta Clem. (Cyanopepla), 126

cruenta Herr.-Schäff. (Burtia), 105

cruentum(-a) Perty (Cosmosoma), 69

crusmatica Dogn., 62

cryptoleuca Walk. (Agyrta), 134 
cryptolcuca Walk. (Saurita), 81 cryptoleuca Hamps. (Saurita), 81 cubana Druce (Phoenicoprocta), 59 cubana Scharis (Dahana), 151 cubense(-is) Schaus, 139 cucadma Druce, 122 cuma Druce, 56 cuprea Schaus (Ceramidia), 115 cuprece Prittw. (Syntomis), 20 cuprcipennis Walk. (Macrocneme), 94 cupreipennis Butl. (Ceryx), 7 cuprizonata Hamps., 13

Currici Dyar, 45 curtiplaga Mab., 13 cyanea Butl. (Macrocneme), 95 cyanea Schaus (Trichura), 100 cyaniris Hamps., 149 cyanitis Meyr., 89 cyanopasta Dogn., 115 cyanura Meyr., 28 cybelistes Holl., 5 oyclopea Ragusa, 17 cyllarus Druce, 95 cymatilis Swinh., 20 cysea(-us) Cram. 20 cysscoides Butl., 20 cytogaster Holl., 9

dacmonis Druce, 112

dalsa Schaus, 52

daltha Druce, 102

damalis Holl., 45

damarensis Grüberg, 14

daphaona Hamps. (Balacra), 45

daphaena Hiamps, (Phia), 61 darantasia Druce, 139

dares Craiu., 136 ins

Davidi Dogn. (Eu^ereum), 139

Davidi Pouj. (Syntomis), 23 deceptans Zerny, 48

deceptrix Hamps., 50

decipions Rothsch., 34

decisa Walk., 64

decora Oberth., 45

decorata Walk., 6

decorum Schaus, 139

deflocea Swinh., 26

delecta Butl., 79

delectans Schaus, 132

demantria Druce, 69

demonnassa Druce, 82

demona Schaus, 77

demonis Druce, 112

deritata Walk., 20

desperata Walk., 63

determinatum(-a) Butl., 69

detola Swinh., 34

detracta Walk., 11 devisum Walk., 149

dexamenc Druce, 56

Deyrollei Herr.-Schäff. (Mallod.), 59

Deyrollei(-ii) Walk. (Cosmosoma), 69 diana Druce, 124

diaphana Dogn. (Saurita), 81

diaphana Koll. (Syntomis), 20

diaphana Sepp (Loxophlebia), 62

dichotoma Leech, 20

dieides Prittw., 106

diffissa Grote, 121

diffusa, Schaus, 81

diffusihelvola Schultze, 88

dilatata Sncll., 21

dilateralis Hamps., 14

diminuta Walk., 83

diplaga Hamps., 11

diptera Fabr. (Ceryx), 6

diptera Walk. (Melisa), 47

discalis Druce (Dclphyre), 135

discalis Schaus (Epcetoptera), 151

discalis Walk. (Cacostatia), 124

discata Druce, 14

discifera Zerny (Fuchromia), 87

discifera Walk. (Loxophl.), 62

discinota Moore, 32

discolor Walk., 139

discophora Dogn,, 6I

discoplaga Schaus, 109

discopuncta Hamps., 146

discosticta Hamps., 151

disrupta Moore, 21

dissimilis Beth.-Bak., 14

disticta Hamps., 125

distinctus(-a) Rothseh. ('Teucer), 111

distincta Saalm. (Thyrosticta), 36

distorta Rothsch., 4

divalis Schaus \& Clem., 14

diversa Beth.-Bak., 42

diversipennis Walk., 113

divisa Walk. (Ctenucha), 149

divisa Walk. (Syntomis), 21

divisa Walk. (Pezoptera), 101

divisura Walk., 11

dixanthia Hamps., 100

dizona Druce 135

dodaba Dyar, 112

Doeri Schaus, 106

Dohertyi Druce (Euchromia), 89

Dohertyi Hamps. (Callitomis), 4

Dohertyi Rothsch. (Ceryx), 7

Dohertyi Rothsch. (Eressa), 34

Dohertyi Rotlisch. (Hyalaethea), 34

Dohertyi Rothsch. (Pseudoceryx) 34

dolens Schaus (Heliura), 137

dolens Schaus (Psilopleura), 79

dolens Walk. (Chrostosoma), 64

dolosa Walk., 116

domimicensis Rothsch., 112 
Doncasteri Rothsch., 58

dorata Dogn., 125

doris Druec, 63

dorsalis Walk., 65

dorsilineata Hamps., 102

dorsimacula Rothsch. (Mesoth.), 63

dorsimaculum(-a) Schaus (Cosm.), 69

dorsipunctum(-a) Hamps., 139

Drucei Hamps. (Lycomorpha), 147

Drucei Rothsch. (Isanthrene), 53

Druce Kirby (Eucereum), 141

Drucei Kirby (Phia), 61

Druryi Hübn., 100

dubia Röber, 89

dubiosa Schaus, 83

dubium Rothsch., 69

ducalis Mrass., 125

dulinfieldia Schaus, 52

dulcicordis Dyar, 76

durea Schaus, 69

duronia Druce, 52

Duvauceli Boisd., 4

dux Walk., 134

dycladioides Heyl., 109

dyschlæena Turner, 29

eacus Cram., 94

eagrus Craun., 99

eburneifera Feld., 102

echemon Druce, 53

echemus Cram., 64

ecliptis Mcyr., 29

ectomelacna Hamps., 56

ecuadorum(-a) Druce, 50

editha Walk., 149

Edwardsi Butl. (Syntomis), 21

Edwardsi Grote (Lymire), 120

efulensis Holl., 40

egenaria Walk., 21

egregia Schaus, 62

Ehrmanni Holl., 45

Ehrmanni Hamps., 45

eion Druce, 129

Ekeikei Beth.-Bak., 89

elachista Holl., 9

elasson Holl., 6

elegans Auriv. (Balacra), 45

elegans Butl. (Cosmosoma), 69

elegans Druce (Correbidia), 146

elegans Druce (Eurota), 84

elegans Druce (Phia), 61

elegans Lathy (Delphyre), 135

elegantissima Wallengr., 88

elegantula Herr.-Schäff., 60

elisa Buti. (Syntomis), 21

elisa(-za) Klages (Aethria), 102

elongata(-us) Dogn. (Correhia), 145

elongata Druce (Hypocladia), 105 elongata Hamps. (Syntomis), 21

elongatus(-a) Schaus (Ptychotr.), 135

elongimacula Hamps., 11

elvira Pagenst., 28

Elwesi Rothsch., 21

emarginata Walk., 125

cmergens Walk., 66

emerentia Dogn., 137

emma Butl., 21

enagrus Cram., 56

endochrysa Dogn., 66

cndocrocis Hamps., 14

endodasia Hamps., 112

cndoleuca Dogn. (Holophaea), 78

endolcuca Druce (Mesothen), 63

cntella Druce, 69

cone Hübn., 76

epa Beth.-Bak., 89

epilais Walk., 91

epimetheus Schaus, 63

opona Dogn., 56

cra Swinh., 21

erebina Butl., 22

elessoides Hamps., 33

Ericssoni Rothsoh. (Eresse), 32

Ericssoni Rothsch. (Ceryx), 7

eriphia Fabr. (Antichloris), 116

eriphia Druce (Ceramidia), 114

Erlangeri Pothsch., 42

errans Hübn., 94

erubeseens Butl. (Cosmosoma), 75

crubescens Hamps. (Tritonnclia), 39

erubescens Walk. (Metarctia), 43

crythraema(-haema) Hamps., 63

erythrarchum(-os) Walk., 69

orythrogyia(-guia) Hamps., 81

crythrolcpe(-is) Dyar, 139

erythrosoma Hamps., 33

erythrotela(-us) Walk., 152

elythrozonata Hamps., 57

cschatias Meyr., 30

esmeralda Butl. (Macrocneme), 95

esmeralda Walk. ('Trichura), 101

espia Dogn. 106

ethela Schaus, 63

ethodaea Druce, 69

euchloa Hamps., 118

euchromioides WValk, 129

eucyane Fold., 118

eucyane Feld., 119

eumelis Druce, 70

eumenes Herr.-Schäff., 107

eumenides Newm., 49

eumolphus Cram. (Euchromia), 87

eumolphus Fabr. (Horama), 122

eumonides Druce, 62

eurypon Druce, 104

euryptera Hamps., 21

euryzona Leech, 21 
eusebia Druce, 76

euterpe Herr.-Schäff., 91

evades Cram., 70

evar Pagenst., 8

evelina Druce, 95

Everetti Rothsch. (Eressa), 33

Everetti Rothsch. (Syntomis), 29

exapta Swinh., 7, 8

excavatum(-a) Dogn., 139

eximia Boisd. (Cyanopepla), 127

eximia Herr.-Schäff.(Phoenicopr.), 59

exomelan Zerny, 70

expallescens Saalm., 37

cxpandens Walk., 7

exsul Rothsch., 48

extensa Walk. (Syntomis), 21

extensa Saalm. (Thyrosticta), 36

exul Rothsch., 48

falerina Druce, 93

famula Frr., 38

fantasia Butl., 13

fanum Druce, 139

fasciata Rothsch., 101

fasciatella Mén., 60

fasciolatum Butl. (Sphecosoma), 49

fasciolatum Druce (Pseudosphex), 48

fasciolatum Druce (Sphecosoma), 50

fastuosa Walk., 127

fastuosa Mén., 128

fata Swinh., 7

faveria Druce, 109

Felderi Druce (Isanthrene), 53

Felderi Rothsch. (Leucotmemis), 66

Felderi Rothsch. (Mesolasia), 99

Felderi Zerny (Napata), 118

fenestra Walk., 132

fenostrata Druce (Napata), 118

fencstrata Jord. (Balacra), 45

fenestrata Klages (Poliopastia), 99

fcnestrata Ramme (Syntomis), 17

fenestrata Drury (Syntomis), 25

fenestrata Herr.-Schäff. (Syntom.),22

fenestrata Walk. (Syntomis), 24

fenestratum(-a) Drury (Cosmos.), 70

fenestrina Butl., 65

ferens Schans, 97

ferox Walk., 91

ferrea Butl., 95

ferrigera Druce, 42

fervida Walk., 21

festivum(-a) Walk., 70

fida Hübn., 129

finalis Walk., 102

fininigra Kaye, 62

finitima Wileman, 7

flammea Dogn., 115

flammula Hübn.-Gey., 126 flava Beth.-Bak. (Ceryx), 6

flava Dogn. (Eucereum), 139

flava Wileman (Syntomis), 23

flavata Druce (Correbia), 145

flavata Jones (Horama), 121

flavia Oberth. (Micronaclia), 36

flavia Schaus (Porramya), 51

flavibasis Hamps., 7

flavicans Dogn., 121

flavicaput Hamps., 139

flaviceps Druce (Delphyre), 135

flaviceps Hamps. (Napata), 118

flaviceps Hamps. (Metarctia), 42

flaviciliata Hamps., 42

flavicincta Auriv. (Metarctia), 42

flavicincta Dogn. (Phia), 61

flavicincta Druce (Argyroides), 104

flavicinctata Dogn. 76

flavicinctum(-a) Schaus, 139

flavicollis Druce, 79

flavicollis Druce, 79

flavicornis Druce (Argyroides), 104

flavicornis Drace (Gymnelia), 58

flavicomis Kaye (Pterygopterus),111

flavicorris Fabr. (Isanthrene), 54

flavicosta Herr.-Schäff., 135

flavicostaie Rothsch., 70

flavicostata Druce, 63

flavidorsia Hamps., 117

flavifascia Herr.-Schäff., 51

flavifrons Hamps., 21

flavigutta Walk., 130

flavigutta Butl., 130

flaviguttata Hamps., 21

flavimacula Walk., 45

flavinigra Jones, 62

flavipalpe(-is) Hamps., 106

flavipes Hamps., 104

flavipicta Hamps. (Phoenicop.), 59

flavipicta Schaus (Loxophlebia), 62 flaviplaga Walk., 11

flaviplagia Hamps., 7

flavipunctata Beth.-Bak. (Eress.), 41

flavita Schaus, 70

flavitarse(-is) Walk., 70

flavitincta Hamps., 134

flavivena. Hamps, 43

flaviventralis Dogn. (Cacostatia), 124

flaviventralis Hamps. (Delphyre), 136

flaviventris Diuce (Mesothen), 64

flaviventris Möschl. (Aclytia), 130

flavocincta Guér., 123

flavofasciatum Möschl., 141

flavolaveta Rothsch., 21

flavopunctatum Dogn. (Eucer.), 140

flavosignata Rothsch., 103

flavothoracides Zerny, 70

flavothorax Rothsch., 70

flavum(-a) Dogn., 139 
flora Schaus, 147

florella Butl., 125

florida Joan., 37

florina Butl., 6

Folletii Gray, 87

formicina Swinh., 9

formosa Guér., 86

Formosao Buti., 21

formosana Butl., SS

formosum Dogn., 140

Fortunei de l'Orza, 22

Fortunei Leech, 19

frances Dyar, 112

francisca Butl., 13

fraterna Butl., 88

frigida Burm., 101

frontalis Walk., 59

Fruhstorferi Hamps. (Callitomis), 4

Fruhstorferi Rotlisch. (Syntom.), 22

frustulenta Swinh., 23

fulgens Herr.-Schäff. (Cyanop.), 128

fulgens $\mathrm{Hy}$. Edw. (Lycomorpha), 147

fulgens Lathy (Euchromia), 89

fulgens Neum. (Propyria), 147

fulgida Herr.-Schäff., 127

fulvescens Walk., 10

fulvia Hamps., 43

fulvicauda Butl., 102

fulvicaudata Lathy, 101

fulvicollis Hübn., 146

fulvida Butl., 87

fulvipicta Kayc, 137

fulvipuncta Harnps., 53

fulvisphex Druce, 109

fulviventris Mén., 75

fumipennis Walk., 115

fumosa Schaus, 64

furva Hamps. (Balacra), 45

furva Hamps. (Eressa), 34

fusca Hamps., 43

fuscicornis Wallengr., 5

fuscipes Rothsch., 104

fusiformis Walk. (Hyaleucerea), 152

fusiformis Butl. (Ceramidia), 115

fusiformis Walk. (Ceryx), 8

Fytchei Moore, 7

galatea(-thea) Schaus, 70

galbanum(-n) Schaus, 70

ganssuensis Gr.-Grsh., 16

ganymede Dbld., 89

Garleppi Rothsch. (Cosmosoma), 70

Garleppi Rothsch. (Homoeocera), 52

Garleppi Rothsch. (Pséudosphex), 48 gaudens Walk., 61

gavisa Walk., 83

gaza, Schaus, 70

gelatina Hamps., 22 geminata Schaus (Loxophlebia), 62 geminata Herr.-Schäff. (Mystroc.), 76 geminiguttata Dogn., 97

gemmata Butl. (Euchromia), 89

gemmatum(-a) Butl. (Cosmosom.), 70

gemmifera Walk., 58

gentilicia Sclıaus, 78

gcographica Meyr., 34

geon Druce, 22

georgiensis Rothsch., 35

goorgina Butl., 20

germana Feld. (Syntomis), 16, 22

germana Rothscl. (Balacra), 46

germanus(-a) Rothsch. (Teucer), 111

gigantea(-um) Druce (Androch.), 113

gigantea Druce (Chrysocale), 97

gigantca Druce (Homococera), 52

gigantea Druce (Hyaleuccrca), 152

giganteun Druce (Euccreum), 140

gigas Rothsch. (Callitomis), 4

gigas Rothsoh. (Chrysocale), 97

gilolensis Rothsch., 28

ginorea Swinh., 7

Girardi Dogn., 127

glagoëssa Holl., 46

glaucopis Feld., 111

glaucopoides Walk., 127

glaucozona Druce, 93

gloriosa Jord. (Balaera), 46

gloriosa Walk. (Cyanopepla), 126

gnatula Boisd., 4

gnoma Butl., 112

gnomoides Schaus, 112

Godarti(-ii) Boisd., 7

Goodi Holl., 14

gracile(-is) Klages (Spliecosoma), 49

gracilis Möschl. (Aethria), 102

gracilis Röber (Syntomis), 28

gracula Dogn., 71

graduata Hamps., 22

grandis Druce (Xanthomis), 92

grandis Kaye (Trichura), 101

gravis Hy. Edw., 147

grenadensis Butl., 53

griseldis Druce, 127

Grotei Butl. (Horama), 121

Grotei Hoore (Syntomis), 22

Grotei Pack. (Lycomolpha), 148

grylloides Walk., 141

guacolda Poey, 140

gracolda Hamps., 142

guapila Schaus, 71

guatemalena Druce, 118

Guillemei Oberth., 46

guincensis Fabr., 87

guttulosa Walk., 9

guyanensis Dogn., 95

gya.s Druce, 104 
gymnelioides Zerny, 124 gynaecomorpha (gynamorpha) Hamps., 130

\section{hadassa Druce, 104}

haemalea Holl., 46

hacmanthus Walk., 132

haemapera Schaus, 61

haematica Holl. (Metaretia), 43

hacmatica Perty (Chrostosoma), 64 hacmatobasis(-abasis) Hamps., 77

haematodes Boisd., 126

haematoessa Holl., 43

hamatosphages Holl., 43

hacmatosticta Jones, 61

haemocera(-acera) Hamps., 107

haemopera(-(-apera) Schaus, 61

hacmorrhoa Walk., 131

haemorrlroidalis Fabr. (Phoenic.), 59

haemorrhoidalis Stoll. (Mesolas.), 99

haemorrhusa Hübn., 100

hacmotricha Hamps, 43

Hageni Snell., 7

halteratum(-a) Fabr., 106

halys Crani., 130

hamon Druce, 81

Hampsoni Barnes (Syntomeida), 91

Hampsoni Dogn. (Coreura), 130

Hampsoni Dogn. (Mesolasia), 100

Hampsoni Klages (Cosmosoma), 71

Hampsoni Rothsch. (Episcepsis), 112

Hampsoni Sehrottky (Macroen.), 95 hanga Herr.-Schäff., 71

harpolyce Sehaus, 71

Hartmanni Schaus, 121

heber Cram., 130

heber Walk., 130

hebes Walk., 136

hecale Schaus, 137

hector Stgr., 71

helena Herr.-Schäff., 84

helena Schaus, 85

helotes Druee, 57

helus Herr. -Schäff., 116

helymus Cram., 98

hemileuea Butl., 66

hemiphaea Dogn., 81

hemiphoeniea Hamps., 14

Henrici Rothsch., 29

heptaspila Turner, 29

hereyna Druec, 71

hermione Burm., 84

herodes Druce, 58

herona Druee, 46

Herrichi(-ckii) Butl., 84

hesione Druce, 95

hesperitis MIeyr., 30

hilaris Walk., 74 hilda Druce (Methysia), 116

hilda Ehrmann (Ceryx), 6

hippotes Cram., 40

hirsuta Walk., 92

histrio Guér., 85

Hoegei Druce, 140

Hoffmannsi Rothsch. (Calonotus), 98

Hoffmannsi Rothsch. (Diptilum),106

Hoffmannsi Rothsch. (Histiaea), 93

holophaea Hamps., 76

holoxantha Zerny, 37

homopteridia Butl., 110

Horsfieldi Moore, 87,89

Hosei Rothsch. (Eressa), 33

Hosei Rothseh. (Trichaeta), 11

Huebneri (Hubneri) Boisd,, 22, 29

humerahis Butl., 29

humilis Herr.-Schäff., 109

Humphreyi Rothsoh., 46

hurama Butl., 127

hyalina Frr. (Dysauxes), 39

hyalina Moore (Ceryx), 7

hyalinum Kaye (Eucereum), 140

hyaloptera Dogn., 127

hyalota Meyr., 29

hyalozonus(-a) Feld, 48

hydatina Butl., 23

hymenopteridia Rothseh, 79

hypochilus(-cilus) Hamps., 71

hypochryseis Hamps., 106

hypoleuea Hamps. (Episcepsis), 112

hypoleucus Herr.-Schäff. (Ecd.), 110

hypophaeus(-a) Hamps., 111

ichneumonea Feld. (Rhynchop.), 79 ichneumoneus(-a) Herr.-Schäff.

(Pseudosphex), 48

ichneumoniformis Rothsch., 33

ichneumonoides Rothsch., 71

idda Plötz, 9

ignea Druce, 64

ignicolor Mén., 57

ignidorsium(-a) Hamps., 71

ignita Herr.-Schäff., 97

igniventris Burm., 85

ignotum(-a) Druee, 140

ima Boisd., 125

imaon Cram. (Ceryx), 7

imaon Hamps. (Histiaea), 93

imitata Druce (Desmotricha), 129

imitata Druce (Loxophlebia), 62

immanis Hamps., 95

imogena Butl., 126

impar Walk., 71

impar Druce, 68

imperialis Druce (Cyanopepla), 127

imperialisHerr.-Schäff. (Eupyr.), 125

imperialis Walk. (Epanyeles), 111 
imprinatuun(-a) Walk., 143

improvisa Schaus., 81

impudicum(-a) Schaus., 71

Imrici Druce, 140

inaclia Schaus., 126

inaequalis SnelI., 7

incendiaria Hïbn., 53

incendiaria Druce, 54

incensa Walk., 43

incertia Walk., 81

incipiens Walk, 6

iuconspicua Holl. (Metarctia), 43

inconspicuata Kaye, 62

inconspicuum Lathy (Eucereum), 140

indistinota Butl., 95

inferioris Buti., 93

innotata Dogn. (Epectoptera), 151

innotata Schaus (Aethria), 102

ino Druce, 140

inornata Walk., 112

inspcrata Walk., 66

insueta Swinh., 23

insularis Butl. (Syntomis), 29

insularis Grote (Eunomia), 60

intensa Butl. (Syntomis), 27

intensa Rothsch. (Eressa), 32

intensa Druce (Cosmosoma), 71

intensa Rothsch. (Cosmosoma), 70

intensum(-a) Walk. (Cosmosom.), 71

intercisa Butl., 132

interclusa Walk., 132

intermedia Rothsch. (Balacra), 46

intermedia Druce (Saurita), 81

intermisse Walk., 8

interniplaga Mab., 14

interposita Hamps., 130

interrupta Grünberg (Euchromia), 87

interrupta Walk. (Chlorostola), 123

iuterrupta Wileman (Syntomis), 23

intersecta Hamps. (Paramya), 51

intersecta Walk. (Leucotmemis), 66

interstans Walk., 87

intranotatum Dogn., 140

intricata Walk., 81

invaria Walk., 43

iole Druce, 94

iphimedea Esp., 17

ipomeae Harr., 91

ira Druce, 131

iridis Hamps., 119

irius Boisd., 89

irroratuml(-a) Schaus, 140

irus Cram., 89

isis Boisd, 90

ismene Möschl., 101

isthmia Feld., 132

Jacksoni Beth.-Bak. (Euchromia), 87

Jacksoni Rothsch. (Syntomis), 14 jalapensis Schaus (Macrocneme), 95 jalapensis Neum. (Horama), 121

jamaicensis Schaus, $\mathbf{5 6}$

Jankowskyi Rothsch., 23

Jansonis Butl., 56

joda Druce (Isanthrene), 53

joda Drucc (Syntomeida), 91

johanna Butl., 14

Jonesi Kaye, 48

Jordani Zerny, 58

juanita Neum., 71

jucunda Walk., 127

jucundissina Dyar, 91

julia Druce, 127

julumito Dogn., 109

juneta Hafner, 39

junctura Walk., 133

jynx Hüibn.-Grey., 119

kalidupensis Rothsch., 29

Kannegieteri Rothsch., 11

kashmirensis Rothsch., 39

katima Schaus, 126

Kefersteini Butl., 39

keiensis Rothsch., 9

Kelleni SnelI., 44

Kelleri Klages, 123

Kenredi Rothsch., 14

Kerri Holl., 41

khasiana Butl. (Syntomis), 23

khasiaua Rothsch. (Eressa), 32

Khasiana Rothsch. (Syntomis), 19

Kindermanni Lod., 17

kinensis Hamps., 23

Klagesi Rothsch. (Cosmosoma), 71

Klagesi Rothsch. (Episcepsis), 112

Klagesi Rothsch. (Loxophlebia), 62

Klagesi Rothsch. (Pseudosphex), 48

Klagesi Rothsch. (Psilopleura), 79

Klagesi Rothsch., (Sarosa), 57

Kruegeri Ragusa, 17

Kuehni Rothsch. (Ceryx), 9

Kuehni Rotlisch. (Hyalaethea), 34

Kuhlweini Lef.s 14

laciades Schaus, 95

laconia Druce, 95

lacrimans(-umans) Schaus, 110

lacteata Butl., 81

lacteinota Butl., 120

ladan Druce, 71

ladas Schaus, 140

lades Cram., 95

laennus Walk., 56

laetifica Maass., 84

lagosensis Hamps., 14

lamia Butl. 112 
lamia Druce, 112

lamia Druce, 112

lampetis Turner, 29

lanceolata Walk. (Loxozona), 121

lanceolatus Butl. (Pseudosphen.), 123

Langleyi Klages, 102

laomedia Druce, 20

lasara Pagenst, 32

lasiophlebia(-iphlebia) Dogn., 81

latenigra Butl, 61

latens Schaus, 81

latera Druce, 132

lateralis Boisd. (Syntomis), 14

lateralis Klages (Phia), 61

laterimacula Zerny, 8

lateritia Herr.-Schäff., 43

latioinctus(-a) Hamps., 48

latifascia Walk. (Trichura), 101

latifascia Butl. (Eucereum), 141

latifascium(-a)Walk. (Eucereum), 140

latilines Walk., 66

latimarginata Hamps., 58

latreillians Kirby, 151

Latreillii Boisd, 24

laudabilis Druce, 151

laudamia Druce, 103

laura Butl. (Euchromia), 88

laura Hamps. (Philorus), 152

laurcola Druce, 46

laurium(-a) Druce, 104

Lecohi Rothsch. (Syntomis), 23

Leechi Drnce (Androcharta), 114

leimacis Holl., 14

lelex Druce, 119

lelex Druce, 118

lemapicta Fourer., 38

Lemoulti Rothsch. (Cosmosoma), 71

Lemoulti Schaus (Eucereum), 140

lena Schaus, 119

lenaeus Cram., 112

lenaeus Druce, 113

lenaeus Druce, 113

leonis Butl., 87

leopardina Rothsch., 36

lepcha Mooro, 33

leprotum(-a) Druce, 140

leria Druce, 140

lerioidos Schaus, 140

lethe Fabr., 87

leucacma Meyr., 29

leucarpis Cram., 102

leucc Maass., 57

leucerythra Holl., 14

leucogastra Holl, 41

leucoma Leech, 25

leucomelas(-a) Walk., 111

leuconotum(-on) Hamps., 72

lencophaea Druce, 139 leucophaeum(-a) Walk., 140

leucoprocta Dogn., 153

leucosoma Butl., 4

leucostalacta Burm., 52

leucosticta Druce (Hyaleucerea), 153

leucosticta Hübn. (Psoloptera), 84

leucostigma Perty, 05

leucotela(-us) Butl., 119

leucozona Hamps., 23

leucozonoides Rotscli., 23

leugalea Holl., 5

libanotica Bang-Haas, 16

libera Walk., 7

lichas Fabr., 122

lichas Cram., 122

ligata Ill., 17

lignicolor Rothsch., 75

limacis Holl., 14

linearis Walk., 26

lineatum(-a) Dogn., 140

litoralis Rothsch., 112

longipennis Noum. (Plineo), 97

longipennis Rotsch. (Calonotus), 98

longipennis Walk. (Syntomis), 23

longipes Herr.-Schäff., 6

lophocera Druce, 52

lophura Auriv., 47

lophuroides Oberth., 47

lucania Schaus, 131

lucens Dogn., 72

lucerna Wileman, 23

lucetius Cram., 86

lucia Oberth. (Dysauxes), 37

lucia Schaus (Cosmosoma), 74

lucina Butl., 23

lucinia Drucc, 125

lucis Butl., 137

lucta Lucas, 29

luctuosa Möschl. (Hyaleucerea), 153 luctuosa Oberth. (Thyrosticta), 37

lugens Jones (Holophaea), 78

lugens Oberth. (Thyrosticta), 37

lugens Röber (Ceryx), 8

lugubris Schaus, 153

lurlina Butl., 90

lutea Grote (Ctenucha), 149

lutea Holl. (Motarctia), 43

luteifascia Hanıps., 23

luteoscapa(-us) Neum. \& Dyar., 150

lutetia Druce, 140

lutibasis Hamps., 57

lutuleuta Snell. (Eressa), 33, 34

lutulentum Möschl. (Eucereum), 144

lycoides Walk., 145

lycoides Oberti., 145

lycone Druce, 78

lycopolis Druce, 72

lydia Druce (Argyroides), 104

lydia Druce (Dycladia), 86 
lydia Druce (Phoenicoprocta), 60 lydia Swinh. (Eressa), 32 lyreca Druce, 58 lysimachides Druce, 130 lystra Druce, 128

macella Dogn., 136

machile Herr.-Schäff., 54

Macgregori Schultze, 8

macroplaca Meyr., 30

macrospila Walk., 5

maculifera Wallengr., 44

maculosa Hamps., 136

madagdscariensis Boisd., 87

madurensis Hamps., 23

magdalene Oberth., 16

magistri Turner., 29

magna Swinh., 88

magnolimbata Dogn., 52

magon Schaus, 104

maja Druce (Eucereum), 141

maja Fabr. (Nacrocneme), 96

maja Burm. (Nacrocneme), 95

major Druce, 117

malaccensis Rothsch., 32

mandarina Butl., 22

maon Drucc, 93

maracandina Ersch., 16

maratha Druce, 65

marcatum(-a) Schaus., 141

marcescens Feld., 32

marelia Butl., 29

margaritifera(-riphera) Butl., 66

marginulis Walk., 14

marginata(-um) Walk., 76

maria Oberth., 35

mariamne Druce, 130

maricum(-a) Cram., 141

marictta Oberth., 35

marina Butl., 14

marita Sohaus, $1+19$

maritañ Schaus, 85

marmoratum Butl., 141

maronicnsis Schaus, 96

Marsdeni Moore, 22

masa Drucc, 63

masia Dogn., 128

Masoni MIoore, 23

Mathami Rothsch. (Autochloris), 56

Mathani Rothsch. (Cosmosoma), 72

Mathani Rothsch. (Isanthrene), 53

Mathani Rothsch. (Sphecosoma), 50

Mathani Schaus (Eucercum), 141

Mathani Rothsch. (Cosmosoma), 70

Mathewi Butl. (Euchromia), 90

Mathewi Hy. Edw. (Scepsis), 147

mathina Druce, 101

maxima Butl., 53 mecrida Druee, 81

mediorubra Kaye, 81

Meeki Rothseh. (Ceryx), 9

Meoki Rothsch. (Hyalaethea), 34

megaspila Walk., 76

megatorna Hamps., 34

megista Hamps., 23

Meisteri Berg., 79

melaenn Cram. (Isanthrene), 54

melaena Dogn. (Syntrichura), 107

melinena Hamps. (Balacra), 46

melacna Hamps. (Dycladia), 86

melacua Walk. (Syntomis), 23

melalcuca Holl. (Apisa), 41

melaleuca Walk. (Hypatia), 79

melanifera Kaye, 81

melanitis Hübn., 112

melanobasis Druce, 100

melanocephala Walk., 120

ruelanocera Hamps., 24

melanochloris Sepp., 116

melanogen Dyar, 49

mclanoperas Hamps.(Eucereum),141

melanoperum Dogn. (Sphecos.), 50 mclanoperum(-a) Hamps. (Cosmosoma), 72

molanoproctis Hamps., 24

melanora Meyr, 9

melanosoma Hamps., 101

melanota Hamps. (Episcepsis), 112

melanota Hamps. (Mlcthysia), 116

melanothoracium Klages, 72

melanotum Hamps. (Sphecosom.), 50

melinthus Cram. (Pseudonya), 77

melanthus Cram. (Syntomeida), 91

melapera Dogn., 50

melaproctis Hamps., 24

mclas Cram. (Isanthrene), 54

melas Walk. (Svntomis), 23

melas Walk. (Trichura), 100

melathoracia Klages, 72

Meldolate Butl., 93

melina Herr.-Schäff., 109

melinda Dyar, 128

melinos Mab., 43

melissa Schaus, 50

melita Druce, 78

molitospila Turner, 30

melitte Möschl., 68

mellina Herr.-Schäff., 109

mellita Schaus, 109

meua Hübn., 99

menephron Druce, 104

mennisata Dogn., 149

meones Cram., 113

meonioides Herr.-Schäff., 114

meres Druce, 74

meridense Rothsch. (Cosmos.), 72

meridensis Schaus, (Sarosa), 57 
meridionale Schrottky, 50

merra Wallengr., 99

merula Dogn., 141

meruloides Schaus, 141

Mestrali Bugn., 17

mctachrysca Druce, 60

metallescens Mén., 72

metallescens Druce, 75

motallicumRothsch. (Cosmosom.), 72

metallicus Druce (Calonotus), 98

metalobum(-a) Hamps., 141

metamcla Hamps. (Sphecosoma), 50

metamelaena(-mela) Dogn. (Napat.), 119

metamclacna(-mela) Dogn. (Loxophlcbia), 63

metamelaena(-melas) Walk. (Lymire), 120

metan Pagenst., 9

metaphaca Drucc (Hyalaethea), 35

mctaphaca Hamps. (Rhychop.), 70

metaphnea Hamps. (Syntomis), 30

metarctioides Hamps., 41

metaxantha(-us) Walk., 107

metcrythra Hamps., 110

meteus Cram., 44

motcus Walk., 42

metocdesis(oidesis) Hamps., 141

mexicana Walk., 60

micans Herr.-Schäff., 128

midas Butl., 24

micilia Cram., 134

microchila(-us) Hamps., 33, 34

militaris Butl., 105

mimica Butl. (Schasiura), 124

mimica Druec (Pseudopompilia), 123

minceus Cram., 5

miuerva Schaus, 85

miniata Pask., 148

minima Druce (Correbia), 145

minima Grote (Pseudomya), 78

minor Butl. (Isanthrenc), 54

minor Rothsch. (Ceryx), 9

minor Rothsch. (Pseudonacha), 36

munor Schaus (Pscudaclytia), 117

minor Warr. (Syntomis), 24

minuta B.-Haas (Syntomis), 17

minuta Boisd. (Thyrosticta), 37

minuta Druce (Argyroides), 104

minuta(-um) Möschl. (Delphyre), 136

minutum Druce (Eucereum), 141

miozona Hamps., 15

misa Strand., 40

miserabilis Holl., 9

misitra Schaus, 96

mitigata Walk., 143

mizar Druce, 141

mochlotus Meyr., 30

modesta Butl. (Atyphopsis), 117 modesta Krulik. (Dysauxes), 39 modesta Schaus (Homoneuron.), 102 moerens Oberth., 37

mogadorensis Blach., 16

molanna Wallengr., 11

Moloneyi Druce, 112

monedula Wailengr., 15

monoleuca Hamps., 11

monoplaga Druce, 134

monotica Holl., 42

monotona Dyar, 136

monstrabilis Klages, 101

montana Boisd. (Thyretes), 40

montana Butl. (Syntomis), 24

montana Schaus (Mesothen), 64

Monteiroi Butl., 40

monticola Auriv. (Syntomis), 15

monticola Klages (Histiaea), 93 monticola Schaus (Isanthrene), 54 Moorei Butl., 32

mora Druce (Saurita), 81

mora Schaus (Sarosa), 57

morosa Schaus, 153

mortia Schaus, 149

mosca Dogn., 65

mota Swinh., 8

moza Drucc, 109

mucescens Feld., 57

mucro Zerny, 122

Muirheadi Feld, 24

multicincta Walk., 59

multifaria Walk., 149

multifasciata Hamps., 4

multigutta Schaus (Ichoria), 77

multigutta Walk. (Eressa), 33

munda Walk., (Abrochia), 109

munda Walk. (Napata), 119

musa Swinh., 32

musca Schaus, 82

muscella Lab., 36

musicola Cockerell, 115

myodes Guér., 35

myrina Druce, 141

myrodora Dyar, 72

myrrha Druce, 81

myrrhine Burm., 153

myrtusa Druce, 141

mysia Druce, 64

naclioides Feld. (Eressa), 34

naclioides Feld. (Psychothoe), 4

naenia Druce, 78

nana Druec (Metaloba), 84

1!ana(-um) Schaus (Mesothen), 64

nantana Walk., 150

Natalii Boisd., 15

nautana Walk., 150

nebulosum Dogn., 141

neglecta Rothsch. (Euchromia), 90 
neglectus(-a) Boisd. (Philorus), 152 negretina Plötz, 14

negus Oberth., 40

nelea Möschl., 72

nesothetis Meyr., 28

newara Moore, 24

nexa Herr.-Schäff., 66

nicippe Drucc, 73

nigerrima Walk., 76

nigrn Hamps. (Epitoxis), 35

nigra Hamps., (Eressa), 33

nigra Schaus (Delphyre), 136

nigrcscens Rothsch., 75

nigrobusalis Rothsch., 15

nigricauda Dogn. (Eumenogast.), 108 nigricauda Miyake (Syntomis), 22 nigriceps Auriv. (Hetarctia), 44 nigriceps Butl., (Syntomis), 27 nigriceus Hamps. (Sphecosoma), 50 nigricilia Strand, 15

nigricincta Hamps., 85

nigricorne(-is) Fabr. (Cosmos.), 72 nigricorne(-is) Pibl. (Sphecosoma), 50 nigricormis Alph. (Syntomis), 18 nigriferum Dyar, 49

nigripalpia Hamps., 81

nigripennis Auriv., 46

nigripes Heyl., 69

nigritarsia Hanps., 96

nigrivalvata Dogn., 60

nigrozona(-um) Schaus, 78

nitidula Herr.-Schäff., 61

niveifascia Walk., 92

nivosa Walk., 151

nobile(-is) Schaus., 72

noctis Druec, 44

Nolclieni Stgr., 133

nomia Druce, 61

nora Diuce, 152

nordina Schaus, 96

norma Hert.-Schäff., 59

Normani Schaus, 147

nostalis Walk., 15

notabilis Walk. (Eumenogaster), 108

notabilis Walk. (Methysia), 117

notatr Butl. (Correbidia), 146

notata Butl. (Sarosa), 57

notha Schaus, 104

notipennis Butl., 54

notochloris Boisd., 133

notoplagia Hamps., 9

notostictum(-a) Dogn., 72

novercn Schaus, 48

nox Druce (Saurita), 82

ndx Druce (Poliopastea), 98

n'tebi Beth..Bak., 15

nychthemeroides (nyctem.),

Walk., 135

nycteus Cram., 91
Oberthueri Hamps. (Correbia), 145 Oberthueri Rothsch.(Micronaclia),36 obliqua Schaus, 129

obliquistria Hamps., 10

oblitterata Grüberg, 46

obscura Butl. (Ceramidia), 115

obscura Druse (Cyanopepla), 128

obscura Hamps. (Atyphopsis), 117

obscura Klages (Saurita), 83

obscura MIöschl. (Euccreum), 141

obscura Schaus (Correbia), 145

obscura Wallengr. (P'oliopastea), 98

obscura Fabr. (Dysauxes), 38

obscuratus(-a) Hamps. (Phil.), 152

obscuratus(-um) Schaus (Ecd), 110

obscurum M[öschl. (Eucereum), 141

obsoleta Burm. (Episcepsis), 112

obsoleta Leech (Syntomis), 19

obtusa Drucc, 145

occidentalis Puthsch., 90

oceola Dyar, 129

ochracea Feld. (Saurita), 82

ochracea Walk. (Balacra), 46

ochrea Hamps. (Micragra), 133

ochrea Mill. (Dysauxes), 39

ochrejpuncta Hamps, 24

ochroopunctata Pagenst., 30

ochreiventris Dogn., 78

ochroscrpa(-us) Grote \& Rob., 150

ochrotum(-a) Hamps., 142

Ochsenheimeri Boisd., 10

ocina Druce, 103

Ockendeni Druce (Eucercum), 142

Ockendeni Druce (Mesothen), 64

Ockendcni Rothsch. (Cosmos.), 72

Ockendeni Rothsch. (Episceps.), 113

Ockendeni Rothisch. (Gymmelia), 58

Ockendeni Rothech. (Poliopast.), 98

odyneroides Walk., 75

oedipus(-ppus) Boisd., 121

oenone Butl. (Euchromia), 90

oenone Butl. (Syntomis), 20

ogovensis Holl., 14 .

olinda Swinh., 29

omole Drucc, 66

omplate Hübn., 68

omphale Harr., 72

opaca Walk. (Pliaeosphecia), 47

opacus(-a) Boisd. (Philorus), 152

opalizans Rothsch., 98

opalocincta Druce, 108

ophion Walk., 104

opponens Walk., 117

opulenta Walk., 97

oratha Druce, 72

orathidium(-a) Druce, 72

orbiculifera Zerny, 18

orbona Druce, 128

orientalis Butl., 88 
ornata Druce (Ceramidia), 115 ornata Mén. (Mesolasia), 106 ornatula Walk., 66 oroyanum Rothsch., 73 orphana Suell, 24 orphnae Turner., 30 ortona Druce, 104 ossa Druce, 124 Owstoni Rothsch., 24 ozora Druce, 37

Pockardi Grote, 147 pactolina Walk., 30 Pagenstecheri Röb., 89 Palaestinae Hamps., 17 palanana Semp., 24 pallada Druce, 142 pallens Beth.-Bak. (Metarctia), 44 pallens Hy. Edw. (Scepsis), 146 pallida Hamps., 44 pallota Plötz, 41 palmeira Schaus, 150

Palmerii Pack., 148 pampa Druce, 62 panacen Druce, 116 panamensis Druce, 128 pandemia Druce, 134 panopes Herr.-Seliäff., 74 pantalon(-thalon) Fabr., 121 papayauum Dogn., 142 parachoria Holl., 42 paradelpha Turner, 30 paraguayensis Schrottky, 84 Parambae Rothsch., 73 paranensis Sohrottky, 66 parapauema Dogn., 58 paraula Meyr., 30 parcipuneta Hamps., 105 pareclecla Holl., 43 paremphares Holl., 44 Parishi Rothsch., 85 parnassia Möschl., 133 pathenii Fabr., 59 parva Auriv., 11 parvigutta Christ., 39 parvipennis Butl., 113 pasca(-us) Leech, 24 passalis Fabr., 24 patagiata Burm. (Eurota), 85 patagiatia Dyar (Autochloris), 56 patricia Schaus, 65 patrona Schaus, 142 patulum(-a) Dogn., 142 paula Röb. (Euchromia), 90 paula Schaus (Mesolasia), 100 paulina Walk., 93 pavo Dogn., 98 peclinata Walk., 110 pectorahis Walk., 24

pelewana Swinh., 90

pellucida Schaus (Chrostosoma), 65 pellucida Schaus (Saurita), 82

pellucidum(-a) Lathy (Cosmos.), 73 pelopia Druce, 109

pelor Druce, 54

Pembertoui Rothsch., 15

penates Druce, 52

pennata Möschl., 102

pennipcs Grote, 121

pentagona Schaus, 54

pentazonatu Hamps., 25

Pentheri Zerny, 79

peralta Schaus, 63

peratea Dogu., 58

Perbosei(-ii) Guér., 54

perconstrictum Zerny, 50

perfenestratum Dyar, 73

perflava Kaye, 64

perilla Druce, 128

perirroratus(-a) Hamps., 152

perixanthia Hamps., 25

perpetua Oberth., 37

perpusilla Walk., 42

Perroli Oberth., 38

persica Koll., 17

persimilis Leech, 25

perspicua Schaus (Cyanopepla.), 128 perspicua Schaus (Saurita), 82

perspicua Walk. (Xanthoplcura), 12*

perstriatum(-a) Hamps., 142

Pertyi Herr.-Schäff., 54 peruviana Schaus, 142 petosiris Druce, 64

petra Schaus, 130

Pfeifferae Moore, 25

Pflumeri Wacquant, 18

phacobasis Hamps., 15

phaeochyta Turner, 30

phaeophlebium(-a) Hamps., 142

phaeoproctum Hamps., 142

phacoptera Hamps., 44

phaeosoma Druce (Heliura), 137

phaeosoma Hamps. (Hyaleucer.), 153

phaeozona, Zcrny, 15

phalerina Druce, 93

phautasiae Butl., 13

phasma Butl., 40

phegea L., 17

phegens Esp., 18

phemonoe Hübn., 116

phemoroides Möschl., 115

phepsalotis Meyr., 30

pheres Cram., 73

pheres Walk., 72

philippinensis Hamps., 25

philocles Druce, 106

phlegmon Cram., 98 
phoenicia Hamps. (Cyanopepla), 128 phoenicia Hamps. (Syntomis), 15 phoenicophorum(-a) Dogn., 73 phoenicosticta Hamps., 82 phoenicozona Hamps., 25 pholus Drury, 148 phyleis Druce, 52 phylla Druce, 134 pica Walk., 142 picta Druce (Paramya), 51 picta Helr.-Schäff. (Eurota), 85 picta Schaus (Pseudomya), 78 picta Walk. (Loxophlebia), 63 pjeticeps Hamps., 153 piciula Walk., 85 pierus Cram., 137 pilati(-ii) Walk., 142 pilosa Rothscl., 58 pinocha Dogn., 97 pitthea Druce, 58 placida Druce (Argyroides), 104 placida Druce (Syntrichura), 107 plagiata Rothsch. (Gymnelia), 58 plagiatum(-a) Rothsch.(Cosmos.), 73 plagosa Swinh., 90

platyleuca Walk., 106

platyzona Feld., 103

plebccula Dogn., 73

plebeja Herr.-Schäff., 97

pleuraemata Hamps., 66 pleurasticta Hamps. (Ceryx), 8 pleurosticta Hamps. (Ceryx), 8 pleurosticta Hamps. (Eressa), 32 plumalis Hamps., 33 plumbea Hamps., 99 plumbicollum Hamps., 142 plumipes Drury, 121

plumosa Rothsoh., 58 plumulatus Klages, 98 plutoniuin(-na) Schaus, 73 polia Druce, 79 polidamon Cram., 28 polistes Hübn., 48 politule Swinh., 32 polusca Drucc, 31 polybia Kaye, 49 polybioides Burm., 48 polymena I., 88, 90 polymita Sparm., 25 polyzonata Hamps., 27 pometinum(-a) Druce, 142 pompilinı Butl., 57 pompiloides Walk., 54 ponga Plötz, 10 pontia Druce, 82 popayana Dogn., 150 porphyria Cram. (Agyrta), 134 porphyria Walk. (Isanthrene), postflava Schaus (Metacrocea), 144 postflava Guér. (Corematura), 101 postflavia Druce (Loxophlebia), 63 postica Walk. (Abrochia), 109 postica Walk. (Pompiliodes), 51 potentia Druce, 83 Pratti Beth.-Bak. (Euchromia), 90

Pratti Leech (Syntomis), 25 prelellus Herr.-Schäff., 121 pretiosa Burm., 128 pretus Cram., 122 Prcussi Auriv., 46 principalis Walk., 97 Prittwitzi Möschl., 107 procridia Hamps., 35 profusa Hamps., 54 projecta Dogn., 150 proleuca Hamps. (Trichaeta), 12 proleucum(-a) Druce (Diptilum), 106 prometina Druce, 78 propylea. Druce, 83 proserpina Hübn., 93 prosomoea Turner, 30 protum(-us) Druce, 73 Pryeri Hamps., 25 pseudarchias Hamps., 142 pseudextensa Rotsch., 25 pscudopolybia Schrottky, 108 pseudosphecia Hamps., 108 pseudothia 'Zerny, 73 psittacus Schaus, 125 pterophorina Mab., 11 ptychoglene Hamps., 147 pudicum(-a) Druce, 73 puella Boisd., 36 pugione I., 122 pulchella Klages, 62 pulchra Auriv. (Balacra), 46 pulchra Dyar (Lycomorpha), 148 pulverea Hamps., 44 pumila Hamps., 44 puncta Druce, 9 punctata Butl. (Aclytia), 130 punctata Fabr. (Dysauxes), 38 punctata Semp. (Syntomis), 25 punctata Guér. (Horama), 121 punctatum(-a) Guér. (Eucereum), 142 puncticincta Holl., 10 puriscal Schaus, 116 purpurca Lathy, 116 pusilla Butl. (Delphyre), 136 pusilla Rothsch. (Trichura), 101 pusilla Butl. (Macrocneme), 94 pydna Druce, 130

pygmula Oberth., 5 pylotis Möschl., 149 pyrama Dogn., 61 pyrgion Druce, 63 pyrocera Hamps., 54 
pyrocoma Meyr., 30

pyroperas Hamps., 136

pyroproctis Druce, 123

pyrozona Druce (Delphyre), 136

pyrozonum(-a) Hamps. (Eucer.), 143

pyrrha Schaus, 64

pyrrhodera Meyr., 23

pyrrhorhoea Hiubn., 108

pyrrhosoma Butl., 137

pyrrhostethus Butl., 75

pytna Druce, 73

quadricolor Fcld. (Cyanopepla), 128 quadricolor Walk. (Antichloris),116 quadricolor Walk. (Eucereum), 143 quadricolor Walk. (Mevania), 133 quadrifascia Hamps., 25 quadrigutta Walk., 77

quadriguttata Dogn., 97 quadrimacula Mab., 37 quadrimaculataN[öschl.(Napata),119 quadrimaculata Zerny (Apisa), 42 quadrioculata Güze, 38

quadriplagata Snell., 12 quadripunctata Dogn. (Chrys.), 97 quadripunctata Rothsch. (Syut.), 25 quadristrigata Hamps. (Napata), 110 quadristrigata Walk. (Dixophl.), 77 quadruplex Dogn., 97

Quartzi Klages, 116

quercue Fabr., 17

quindiensis Dogn., 164

quinquecincta Hamps., 33

quinquemacula Mab., 38

quinquepunctatum(-a) Heyl., 73

quisqualis Swinh., 6

rabdonota Hamps., 119

rabusculium Dogn., 143

raca Druce, 145

ragusaria Zickert, 39

Rattrayi Rothsch., 46

reba Druce, 107

recedcus Lucas, 30

reducta, Mab., 36

redunda Schaus, 113

refulgens Dogn., 150

regalis Boisd., 97

regium(-a) Schaus, 73

Reimoseri Zerny, 150

relcgatum Schaus, 143

remissa Dogn., 117

remotum(-a) Walk., 73

Rendalli Dist. (Syntomis), 15

Rendalli Rothsch. (Apisa), 42 .

reniferum Hamps., 143

resecta Herr.-Schäff., 6 resina Druce, 143

restricta Hamps. (Hypocladia), 105 restrictum Butl. (Cosmosoma), 75 reticulatum Butl., 143

Reutlingeri Holl., 14

rhabdonota Hamps., 119

rhodocera Schaus, 52

rhodocrypta Druce, 136

rhodophila Walk. (Heliura), 137

rhodophilaDruce (Eucereum), 137,143

rhypoperas Hamps., 113

Ribbei Druce (Cyanopepla), 128

Ribbei Röber (Syntomis), 25, 31

Rilbei Druce (Trichura), 101

rica Dogn., 73

Rodriguezi Druce, 52

Rogenhoferi Schaus, 64

Rogersi Drucc, 143

rosa Walk., 143

rosacea Bcth.-Bak., 44

rosadora Dyar, 143

rosea Auriv., 44

roseiccps Dogn. (Delphyro), 136

roseiceps Iruce (Atyphopsis), 117

RoscnbergiRothsch.(Cosmosoma), 73

Rosenbergi Rothsch. (Poliop.), 99

Rosenbergi Rotlisch. (Cosmosoma),75 rosina Walk., 143

ruatana Druce (Bombopsyche), 50

ruatana Druce (Holophaea), 78

rubclla Grote, 105

rubicunda Dogn. (Ctenucha), 150

rubicunda Mab. (Syntomis), 14

rubipectus Schaus, 103

rubra Walk, 43

rubribasa Beth.-Bak., 44

rubrobasale(-is) Lathy, 73

rubricauda Dogn., 101

rubriceps IValk., 152

rubricincta Burm. (Androch.), 114

rubricincta Hamps. (Delphyrc), 136

rubricincta Hamps. (Rhynch.), 79

rubricincta Holl. (Balacra), 46

rubricincla Hamps. (Balacra), 46

rubricollis Hamps. (Uraga), 131

rubricollis Walk. (Eucliromia), 90

rubricorpus Kaye, 76

rubriguttum(-a) Skinner, 73

rubrilineata Beth.-Bak., 44

rubripalpus Hamps., 49

rubripcctus Schaus, 103

rubripeda Lucas, 70

rubripuncta Hamps. (Mctarctia), 44

rubripuncta Schaus (Saurita), 82

rubripunctata Butl., 61

rubritarse(-is) Schaus, 73

rubritincta Hamps., 15

rubriventris(-a) Hamps., 60

rubroscapa(-us) Mén., 150 
rubroscapulum(-ac) Kaye, 73 rubrostriata Auriv., 46 rubrothorax Rothsch., 111 rubrovitta Auriv., 46 rubrozonata Ponj., 25 rububa Druce, 139 rufescens Rothsch. (Ioxophleb.),63 rufescens Walk. (Metarctia), 44 ruficeps Walk. (Ctenucha), 150 ruficeps WValk. (Herea), 107 ruficolle(-is) Lathy, 143 rufina Oberth. 15 rufipes Rothsch., 50 rufiventris Schaus, 136 rufogularis Möschl., 117 rypoperas Hamps, 113

sadana Druce, 143 sagada Semp., 31 sages Druce, 125 sahacon Druce, 52 sala Swinh., 26

Salmoni Druce, 97

Salomonis Swinh., 90

salta Schaus, 82

Salvini Butl., 73 samarca Schaus, 128 samina Druce, 64 sanctula Dogn., 103 sandion Druce (Homoeocera), 52 sandion Druce (Xanthopleura), 123 sangaris Wallengr., 22

sanguiceps Drucc (Abrochia), 109 sanguiceps Humps. (Micragra), 133 sanguiceps Hamps. (Pseudomya), 78 sanguioincti Hamps., 119

sanguiflua Hübn.-Gey, 102

sanguifluca. Walk., 102

sanguinaria Streckcr, 149

sanguinea Druec (Saurita), 82 sanguinta Schaus (Argyroides), 104 sanguinea Walk. (Phoenicopr.), 60 sanguinea Druce(Phoenicoprocta), 60 sanguinosa Martin, 122

sanguipalpia Hamps. (Hcliura), 137 sanguipalpis Dogn. (Phara), 135 sanguipuncta Druce (Mallodeta), 59 sanguipuncta Hamps. (Psilopl.), 79 sanguipuncta Schaus (Marec.), 122 sanguisecta Hamps., 82 sanguitarsia Hamps, 109 sapphira (saphira) Stgr., 124 sarama Dogn., 125 sarcosoma Butl. (Acthria), 103 sarcosoma Hamps. (Metarctia), 44 sargania Butl., 8 saron Druce, 74 saturata Walk., 131 saturatissima Walk., 108 saucia Walk., 99

Sauloyi Guér., 92 scapularis Herr..Schäff., 116 scepsiformis $\mathrm{Hy}$. Edw., 152

Schausi Druce (Ixylasia), 124 Schausi Dyar (Propyria), 147

Schausi Hamps. (Eurota), 85

Schausi Rothsch. (Chrostosoma), 65 Schausi Rothsol. (Isanthrene), 54 Schoenerrhi Boisd., 20

Schultzei Auriv., 11

scintillans Druce (Cosmosoma), 68 scintillans Herr.-Schäff. (Cosm.), 70 scintillans Butl. (Cyanopepla), 128 scintillans Rothsch. (Episcepsis), 113 scitum(-a) Walk., 74

Scudderi (-ii) Butl., 116

scyton Cram., 143

sectinotum(-a) Hamps., 74

sectum Kaye, 76

selecta Herr.-Schä,ff., 55

selene Fold., 133

selva Herr.-Schäff., 85

semicincta Hamps., 8

semidiaphanu Harr., 146

semiflava Rothsch., 63

semifulvum(-a) Druce, 74

semifuser Hamps., 33

semihyalina Kirby (Ceryx), 6

semihyalina. Walk. (Pompilopsis), 51 semiluna Walk., 85

seminigra Holl. (Ceryx), 6

seminigra Spul. (Syntomis), 18

semiopaca Dogn., 77

scmirubra(-um) Hamps., 65

semirufa Druce, 80

semistria Walk., 150

semitransvorsa Schaus, 145

semiviridis Druce, 96

semivitreata Hamps., 124

separabilis Walk., 12

separata Bang-Haas, 99

sephela Druce, 74

seraphinum(-a) Hcrr.-Schäff., 74

sericaria Perty, 85

scricaria Herr.-Schäff., 85

sericca Helr.-Schäff. (Saurita), 82

sericeus(-a) Schrottky (Pseudos.), 49

serra Schaus, 56

serrata Hamps., 26

serva Hübn., 38

servula Berce, 39

sesiiformis Walk., 57

sctosum(-a) Sepp, 143

sexmaculata Gianelli, 18

sexpuncta Rothsch., 33

shoa Hamps., 15 
shortlandica Swinh., 90

siamensis Butl., 88

siamica Walk., 33

sibulana Semp., 26

sicula Dyar, 74

siguata Walk., 26

signatura Walk., 131

silacea Plötz, 43

silpha Dogn., 106

silva Schaus, 97

silvius Stoll, 143

simile Schaus (Sphecosoma), 50

similis Heyl. (Callopepla), 126

simillima Rothsch. (Syntomis), 31

simillimumRothsch.(Cosmosoma), 74

simplex Butl. (Micronaclia), 36

simplex Rothsch. (Eressa), 33

simplex Walk. (Autochloris), 56

simplex Walk. (Syntomis), 15

simulans Druce, 56

simulatrix Walk., 130

sinensis Rothsch., 25

singularis Walk., 109

Sintenisi Standf., 18

sippia Plötz, 40

sirius Dist.. 13

sithon Druce, 78

sitiona Schaus, $6 \tilde{}$

sixola Schaus, 113

Sjoestcdti Auriv., 42

Sladeni Moore, 26

smaragdina Walk., 108

Snelleni Rothsch., 26

sobria Schaus, 145

socorrensis Dogn., 63

solicauda Butl., 137

sordida(-us) Rothsch. (Episc.), 113

sordida Walk. (Pezoptera), 101

sororia Schaus, 153

sortita IValk., 59

sparta Druce, 50

spectrum Schaus, 104

speculigera Grünberg, 46

sperans Walk., 61

sperbius Fabr., 26

spenchia(-us) Cram., 87

sphenodes Meyr., 9

sphenophora Turner, 30

spiracula Mén., 92

splendens Butl. (Euchromia), 87.

splendens Dogn. (Chrysocale), 97

splendens Druce (Pseudomya), 78

splendens Möschl. (Loxoplulebia), 63 splendida Butl. (Euagra), 132

spleudida Herr. -Schä.ff. (Napata),119

splendida Butl. (Macrocneme), 95

Steinbachi Rothsch. (Cosmosoma), 74

Steinbachi Rothsch.(Phoenicopr.),60

Steinbachi Rothsch.(Pseudosph.), 49 stellaris Snell., 26

stellifera Butl., 142

stelotis Mcyr., 29

stenipter Hamps. (Rhynchop.), 80

stenoptera Zerny (Syntomis), 15

stenozona Hamps., 31

steropes Göze, 70

stictibasis Hamps., 85

stictoptera Rothsch, 15

stictosoma Druce, ซ2

stigmatica Grünbcrg, 46

stilbostictum(-a) Butl., 74

stipata Walk, 103

strepsimeris Meyr., 34

Stretchi(-ii) Butl. (Androcharta), 114

Stretchii Butl. (Cosmosoma), 72

striata Druce (Correbidia), 146

striatum(-a) Druce (Eucereum), 144

strigivenia Drucc, 120

strigiventris Guér., 85

strigosus(-a) Druce, 49

strigula Druce, 104

stryma Drucc, 74

Stuarti Rothsch., 74

stulta Herr.-Schäff., 64

styx Walk., 83

suapurensis Klages, 105

subapicalis Schaus (Delphyre), 136

subapicalis Walk. (Dinia), 99

subaurata Walk., 33

subcanescens Rothsch., 42

subcyanea Walk., 131

subfenestrata Auriv, 38

subflamma Druce (Rhynchop.), 80

subflammum(-ia) Walk. (Cosmos.), 74

subflavescens Kaye, 119

subformicina Beth.-Bak., 31

subgloriosa Stgr., 127

subhyalina Hamps. 147

sublutca Betll.-Bak., 35

submacula Schaus (Saurita), 82

submacula Walk. (Cyanopepla), 128

submacula Walk. (Microgita), 133

submarginalis Walk., 26

subochrea Herr.-Schäff., 145

subplenus(-a) Walk., 111

suffusa Lathy, 136

sulana Lathy, 31

superba Druce (Agyrta), 134

superba Druce (Napata), 120

superbus Druce (Ptcrygopterus), 111

sura Schaus, 96

surcatųm(-a) Dogn.. 144

surinamensis Möschl., 114

surrentum Druce, 50

Swinhoei Beth.-Bak. (Ceryx), 9

Swinhoei Leech (Syntomis), 19

sylpha Dogn. 106 
sylva Scliaus, 97

sylvicolens Butl., 37

sylvius Stoll, 143

sylvius Sepp, 142

symplionn Swinh., 26

synedra Mcyr., 30

syntomia Plötz, 43

syntomida Stgr., 39

syntomoides Boisd. (Syntom.), 92

syntomoides Butl. (Callitomis), 5

syntomoides Rothsch. (Eressa), 33

syrphiformis Herr.-Schäff., 66

taiwana Miyakc, 26

taos Hamps., 58

tarapotense(-is) Druce, 74

tarona Hamps., 144

tarsalis IValk. (Pompilopsis), 50

tarsalis WValk. (Horama), 122

tarsipuncta Schaus, 58

taurica Hamps., 16

Taymansi Rothsch., 44

teda Walk. 60

tcinopera Hamps., 26

telamonophorum Prittw., 106

telephus Walk., 74

temcuus Cram., 82

temberata Schaus, 64

tenebrose Walk., 51

teneifol'mis Walk., 12

tenellulum Schaus, 144

tenera Mab., 36

tengyra Walk., 75

tenthredinoides(-doides) Walk., 66

tenuis Butl. (Saurita), 82

tenuis But. (Saurita), 80

tenuis Walk. (Syntomis), 26, 31

terminalis Walk. (Ceryx), 5

terminalis WValk. (Correbidia), 146

terminalis Walk. (Napata), 119

terra Scluaus, 131

tessellatum Schaus, 144

testacea Auriv. (Balacra), 47

testacea Druce (Delphyre), 136

testaceum Druce (Eucereum), 144

testaceum(-a) Walk. (Sphecosoma), 50

testaceum Druco (Sphecosoma), 49

tetilla Dogn., 136

tetragonaria Walk., 26

tetragonia Hamps., 110

tetragramma Walk., 137

tetragramma Walk., 137

tetrazona Hamps., 109

tctrazonata Hamps., 26

teuthras Waik., 75

texana Grote, 122

thatassica Feld.. 141

thelebas Cram. (Euchromia), 89 thelebus Fabr. (Syntomis), 25

thelebus Mén. (Syntomis), 22

thera Druce, 60

thetis L., 113

thia Drucc, 75

thia Schaus, 73

thisbe Yōschl., 137

thomasina Butl., 15

thoos Fabr., 113

thoracica Klages (Saurita), 82

thoracica Moore (Syntomis), 26

thoracica Schaus (Leucotmemis), 66

thoracica Walk. (Psoloptera), 84

tharacica Druce (Psoloptera), 84

thoracicum Scliaus (Cosmosoma), 75

thyestes Druce, 54

thyra Möschl., 96

thyretiformis Wallengr., II

thyria Druce, 106

thyridia Hamps., 96

thysbe Nöschl., 137

Tiandae Hamps., 31

tilvalis Butl., 121

tiburtus Cram., 98

Ticaonis Hamps., 26

tigratum(-a) Herr. -Schäff., 142

tigrina Streck. (Ctenucha), 150

tigrina Walk. (Trichaeta), 12

tigris Schaus, 75

tijuca Schaus, 82

tina Walk., 93

tinopera Hamps., 26

tipulina Hübn., 82

tisanemena Dogn., 85

togata Druce, 150

Tollini Keferst., 37

tolosa Drucc, 52

tolumnorsis Herr.-Schäff., 92

tomasina Butl., 15

torquata(-us) Druce (Gymnelia), 58

torquata(-us) Leech (Syntomis), 26

torrida Walk., 66

toxotes Hamps., 6

trabea Druce, 78

tractipennis Butl., 114

tractipennis Druce, 114

transiens Walk., 86

transitiva WValk., 8

translucida Dogn., 78

triangulifera Druce (Saurita), 83

triangulifcra Fold. (Loxophlebia), 63

trichactiformis Zerny, 40

tricincta Herr.-Schäff., 77

tricingulata Culox., 18

tricolor Feld. (Chrostosoma), 65

tricolor Pack. (Argyroides), 105

tricolor Schaus (Saurita), 83

trifasciuta Butl., 152

trifenestra Röber, 31 
trifida Dogn.. 131

trigonophora Turner, 31

trigutta Druce (Saurita), 82

trigulta Walk. (Pseudomya), 77

trimacula Mab., 37

trinita Schaus, 144

Trinitatis Rothsch., 50

triplagus(-a) Hamps., 98

tripunctata Beth.-Bak. (Syntom.),16

tripunclata Scop. (Dysauxes), 38

tripunctatum Dogn. (Encereum),144

tripunctatus Druce (Calonotus), 98

tripunctatus Hamps. (Calonotus), 98

tristriata Herr.-Schäff., 103

tristigma Mab., 42

tristis Schaus, 136

tristissima Perty, 83

tristitia Kaye, 146

trita Dogn., 129

tritonia Hamps., 16

trivittata WValk., 135

trizona Dogn., 52

tivizonata Hamps., 12

Troetschi Druce, 123

trogonoides Walk., 124

tropica Walks., 118

Trotschi Druce, 123

Tryhanei Rothsch., 54

Tunneyi Rothsch., 31

tyrrhene Hübn., 67

ufentina Drucc, 57

Ugandae Rothsch., 45

umbra Druce, 47

umbraticola Klages, 124

umbrescens Schaus, 151

umbrica Schaus, 117

umbrimacula Schaus, 137

undulata Druce, 146

unicolor Oberth., 44

unifascia Hamps. (Syntomis), 27

unifascia Schaus (Napata), 119

uniformis Betl..-Bak. (Metarctia), 44

uniformis Hamps. (Desmotricha), 129

unimacula Rothsch. (Phaeo), 97

unimacula Schaus(Pscudaclytia), 117

unxa Druce, 65

uranophila Walk., 94

urophora Herr.-Schäff., 100

ursula Cram., 129

ustrina Hübn., 54

ustulata Feld., 114

utica Druce, 61

vacillans Walk., 60

Vandepolli Rothsch., 27

varia Walk. (Eucereum), 144 varia WValk. (Isanthrene), 55

varia Hamps. (Isanthrene), 53

varians Hamps., 136

variegata Kaye, 105

varipes Walk. (Leucotmemis), 66

varipes Walk. (Mystrocneme), 76

varium(-a) Walk. (Eucereum), 144

varuna Druce, 134

velotipennis Walk., 16

velutinum Schans, 144

venata Butl. (Episcepsis), 113

venatum Rothisch. (Cosmosoma), 75

venezuelensis Klages (Napata), 119

venezuelensis Klages (Saurita), 83

venosa Walk. (Ctenucha), 150

venosa Walk. (Metaretia), 43

venosum(-a) Schaus (Eucereum), 144

ventralis Guér., 131

verdivittata Klages, 99

verecunda Swinh., 27

vernana Druce, 71

veronica Oberth., 16

vespa Hamps. (Eressa), 33

vespa Herr.-śchäff. (Phaeosphec.), 47

vesparis Butl., 63

vesparium(-

vespiformis Butl. (Isanthrenc), 55

vespiformis Hert.-Schäff. (Pomp.), 51

vespina Rothsch. (Eressa), 33

vespina Schaus (Argyroides), 105

vespina Harr. (Horama), 122

vespoides Rothsch. (Eressa), 33

vespoides Schaus (Poecilosoma), 76

vespoides Walk. (Isanthrene), 54

vesta Möschl., 7S

vestale(-is) Schaus, 144

vicinum Schrottky, 50

victorina Druce (Procalypta), 131

victorina Plötz (Myopsyche), 10

vidua Mén., 42

Vigorsi Mloore, 12

villia Druce, 75

vinasia Schaus, 113

vindonissa Druce, 83

virens Butl., 107

virescens Möschl., 141

virginica Charp., 151

viridicingulatum Rothsch., 75

viridifusa Schaus, 96

viridipunctata(-um) Rothsch., 65

viridis Druce (Ceramidia), 115

viridis Druce (Poliopastea), 99

viridis Lathy (Eriphioides), 114

viridivittata Klages, 99

vitiensis Hamps., 90

vitrea Schaus (Agyrta), 134

vitrea Walk. (Syntomis), 27

vitreata Herr.-Schäff. (Syntomis), 20

vitreata Rothsch. (Balacra), 47 
vitrina Oberth. (Balacra), 47 vitrina Rothsoh. (Dycladia), 86 vitristriga Druce, 83 vittata Walk. (Macrocneme), 96 vittata Walk. (Psilopleura), 79 vittata Druce (Macrocneme), 96 vittigera(-nm) Blanch., 151 volans Swini., 7

volatilis Schaus, 57

voltumna Druce, 67

vulcana Druce (Syntomeida), 92 vulcanus Walk. (Eurota), 85 vulnerata Butl. (Hyaleucerea), 153 vulnerata Herr.-Schäff. (Cosmos.), 69

Wahnosi Rothsch., 91

Waldowi Grünberg, 16

Walkeri Drucc (Napata), 120

Walkeri Hamps. (Euchromia), 91

Wallacei(-ï) Moore, 27

Walsinghami Hy. Edw., 150

Watkinsi Druce, 52

Watsoni Rothsch. (Cosmosoma), 75

Watsoni Rothsch. (Saurita), 83

Whiteheadi Rothsch., 31

Whitelyi Druce, 56

Wilemani Rothsch., 27

Williarni Rothsch., 16

Wimberleyi Swinh., 27

Woodfordi Butl., 35

Wrighti(-ii) Stretch (Scepsis), 147

Wrighti Gundl. (Syntomeida), 92

waka Pagenst., 31 ,

xanthistis Hamps., 75

xanthobasis Druce (Sarosa), 57

xanthobasis Hamps. (Dycladia), 86 xanthocera Walk. (Nyridela), 65 xanthooerum(-a) Hamps. (Cosm.), 70 xanthodora Dyar, 143 xanthogaster Druco (Herea), 107 xanthogastra Perty (Gymnelia), 58 xanthogastroides Schaus, 56 xanthoma Lcech, 27 xanthomela Walk. (Syntomis), 22 xauthomelan Zerny (Cosmosoma), 75 xanthoperas Hamps., 144 xauthopleura Holl., 10 xanthorrhime Herr.-Schäff., 55

xanthosoma Hamps. (Eurota), 85 xanthosoma Hamps. (Myopsyche), 10 xanthosoma Turner (Syntomis), 31 xanthospila Hamps., 80 xanihostacta Humps., 34 xanthostictum Hamps., 75 xanthotarsis Lathy, 57 xanthozona Dogn., 61 xanthura Turner (Syntomis), 31 xanthurum(-a) Schaus (Eucer.), 144 xenodicc Druce, 129 xenodorus Druce, 06

ypleta Swinh., 34

yunnanensis Rothsch., 27

zamorae Dogn., 143

zantes Heir.-Schäff., 64

zelosum(-a) Dogn., 75

zephyrum Schaus, 144

zethus Hübn,, 110

zeus Schaus, 135

zizana Dogu., 144

zonata Druce, 137

Zurcheri Druce, 75

zygaenoides Mab., 35 


